Molecularly Imprinted Polymers for the Analysis of Small Cell Lung Cancer Biomarkers by Liquid Chromatography-Tandem Mass Spectrometry

Thesis for the degree Philosophiae Doctor

by

Nicholas McKitterick

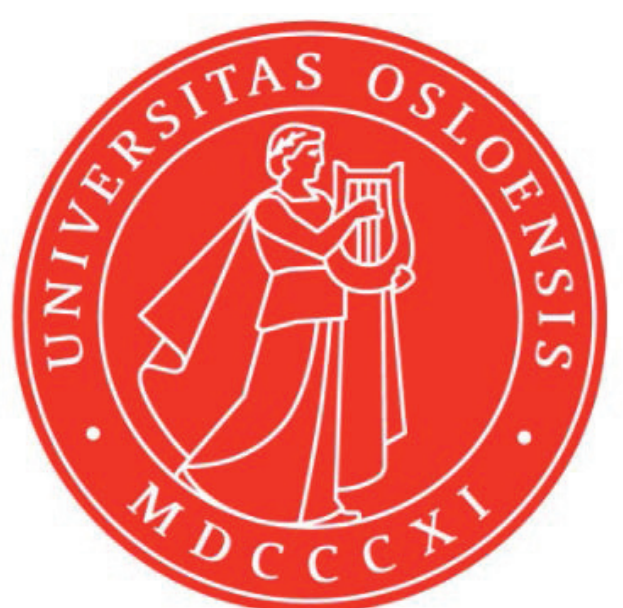

Section for Pharmaceutical Chemistry

Department of Pharmacy

University of Oslo Norway 
(C) Nicholas McKitterick, 2021

Series of dissertations submitted to the

Faculty of Mathematics and Natural Sciences, University of Oslo

No. 2367

ISSN 1501-7710

All rights reserved. No part of this publication may be reproduced or transmitted, in any form or by any means, without permission.

Cover: Hanne Baadsgaard Utigard.

Print production: Reprosentralen, University of Oslo. 


\section{Table of Contents}

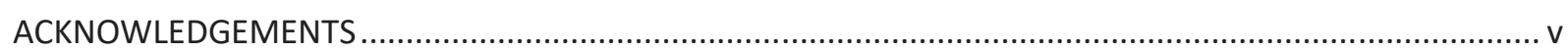

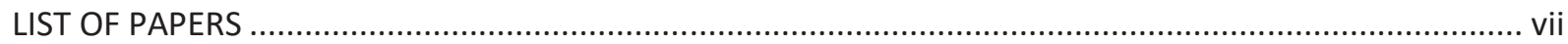

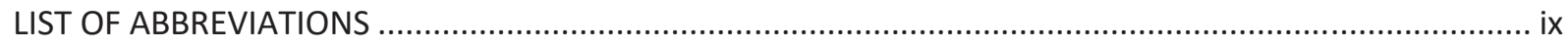

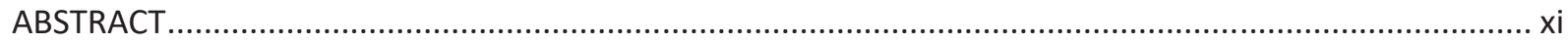

1 INTRODUCTION

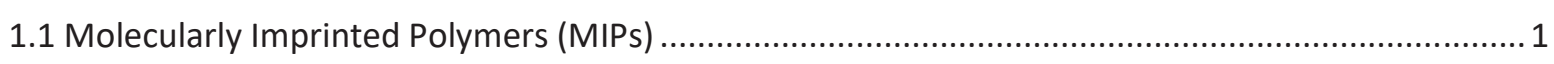

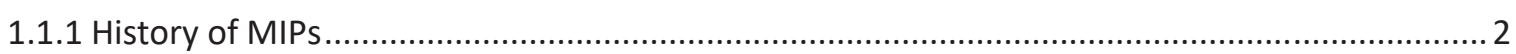

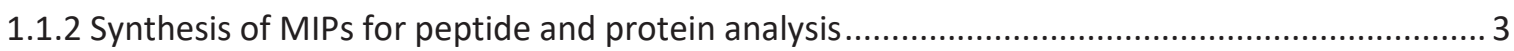

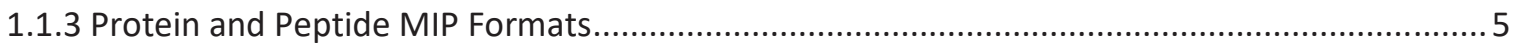

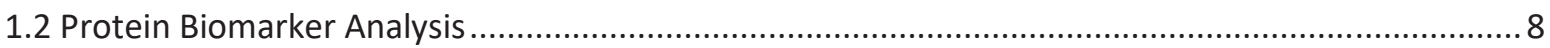

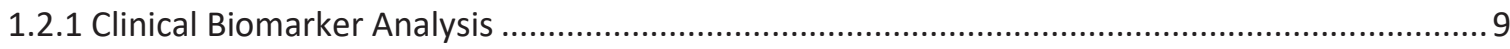

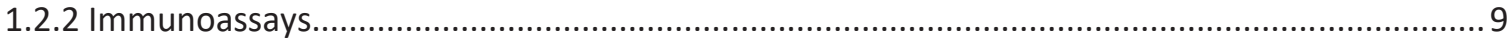

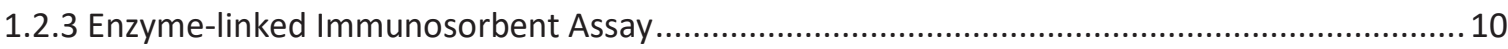

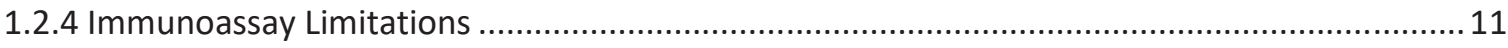

1.3 Liquid Chromatography-Mass Spectrometry (LC-MS) …......................................................... 13

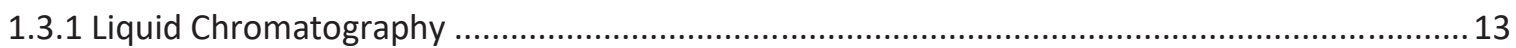

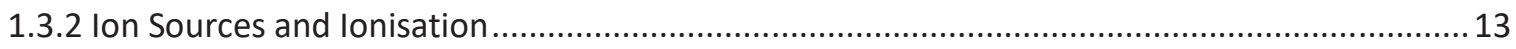

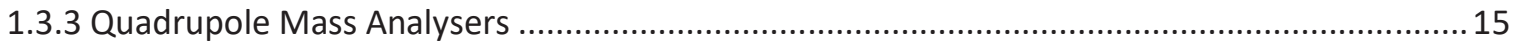

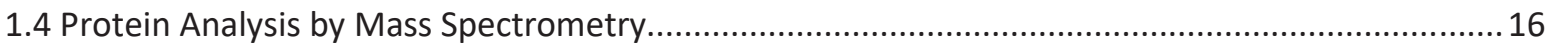

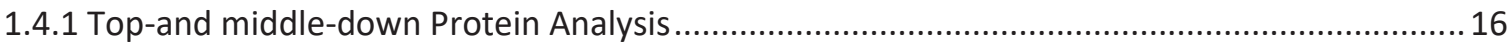

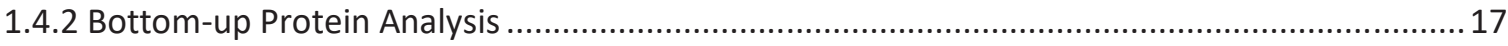

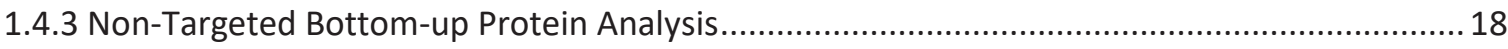

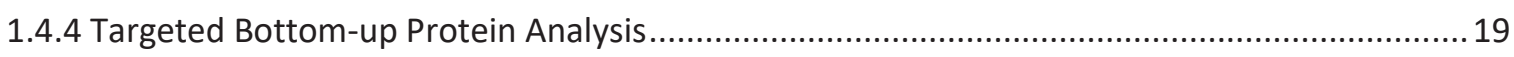

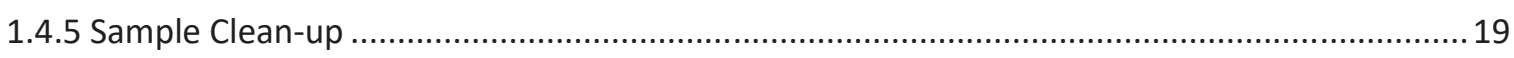

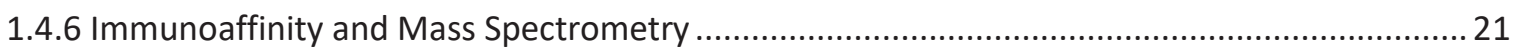

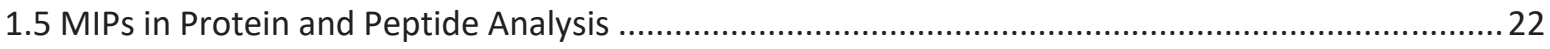

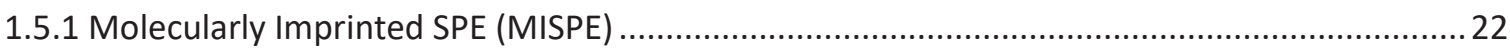

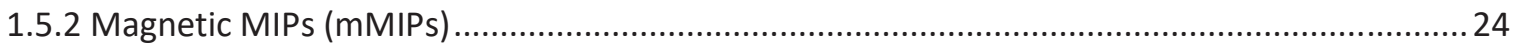

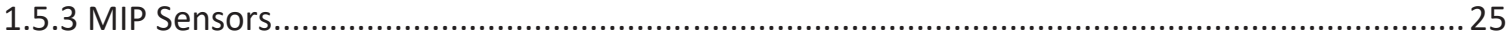

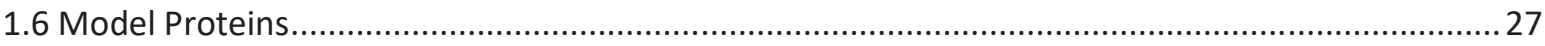

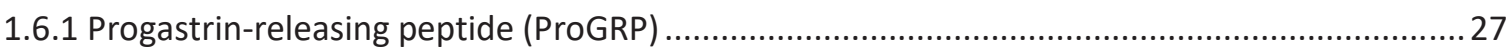




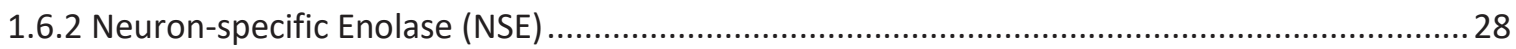

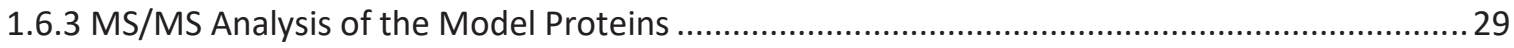

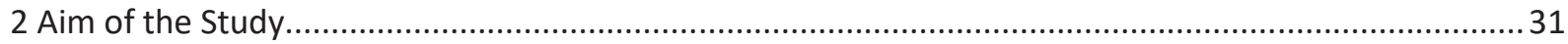

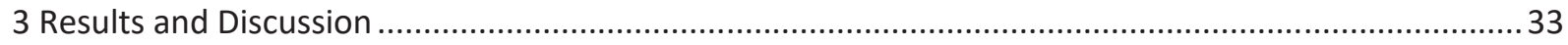

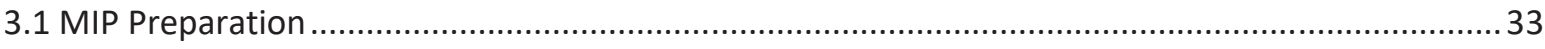

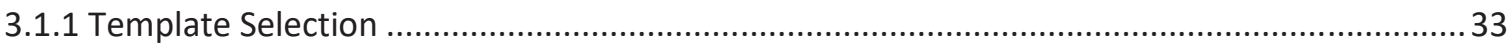

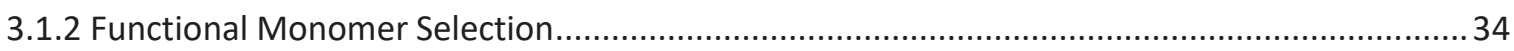

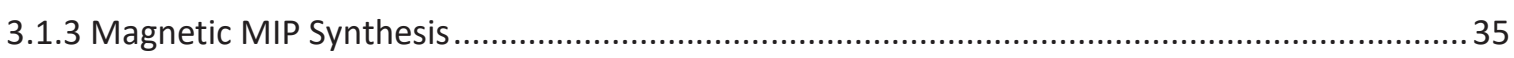

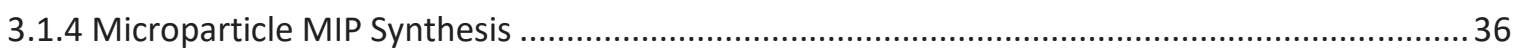

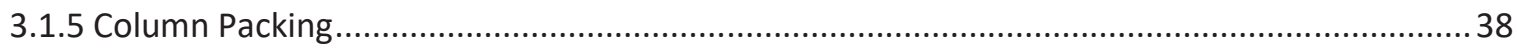

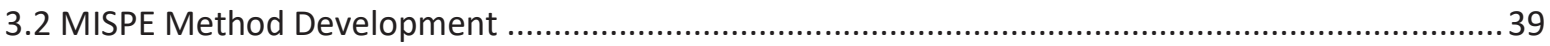

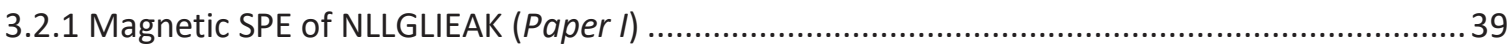

3.2.2 On-line SPE of NLLGLIEAK and ELPLYR (Paper I/ and III) ........................................................... 42

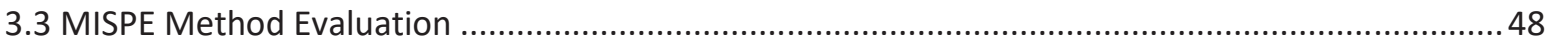

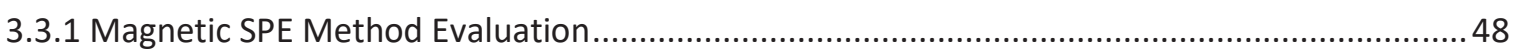

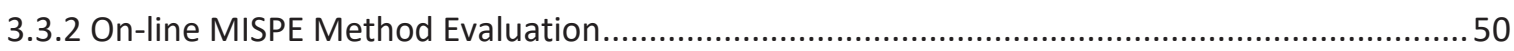

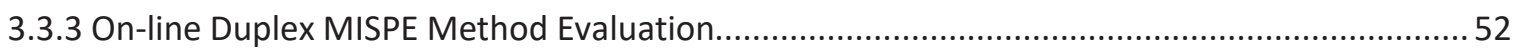

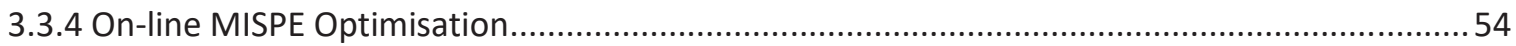

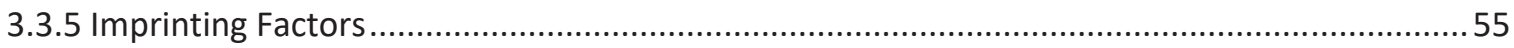

3.3.6 Comparisons of the Single and Duplex on-line MISPE Approaches..........................................56

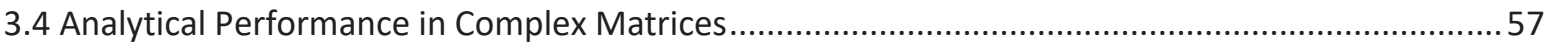

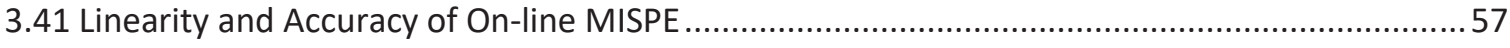

3.4.2 Extraction Recovery, Limits of Detection and Limits of Quantification ......................................57

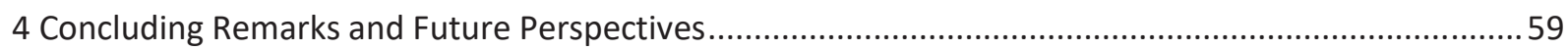

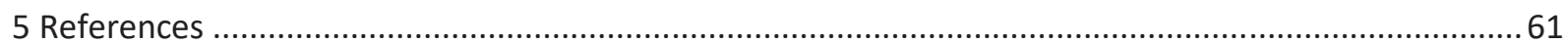




\section{ACKNOWLEDGEMENTS}

The research presented in this thesis was performed at the section of Pharmaceutical Chemistry, Department of Pharmacy, University of Oslo in the period of June 2017 to November 2020.

First and foremost, I must thank my supervisors Trine Grønhaug Halvorsen and Leon Reubsaet for helping me every step of the way and helping me to grow as a person and a scientist. I am especially grateful for their patience, understanding, and optimism. I am proud to be their Ph.D. student.

I would be remiss not to thank my amazing collaborators Peter Cormack and Cem Biçak from the University of Strathclyde, Glasgow. Thank you for producing the materials used in this Ph.D. and for helping me to understand the complexities of imprinted polymers.

To my masters' students, Frida Braathen and Jasna Petrovic, thank you for your invaluable contributions to this work.

To all my colleagues throughout the years, thank you all so much for the amazing work environment, chats, and good times. You helped to create a wonderful work environment. I would like to especially thank Magnus Restan for helping me adjust to life in Norway and always being available for chats about life and football.

To my family, I am thankful for your support and encouragement. I know it has been difficult with me being so far from home. Especially l'd like to thank Mark for sacrificing his Saturday mornings and helping with the graphical abstracts.

Most of all I would like to thank my Zhenya. You have been amazing these last few years. Thank you so much for your love and support.

Oslo, November 2020

Nicholas McKitterick 


\section{LIST OF PAPERS}

I. McKitterick N, Braathen F, Switnicka-Plak MA, Cormack PA, Reubsaet L, Halvorsen TG. Magnetic synthetic receptors for selective clean-up in protein biomarker quantification. Journal of Proteome Research 2020, 19 (8), 3573-3582.

II. McKitterick N, Bicak TC, Cormack PA, Reubsaet L, Halvorsen TG. Facilitating Serum Determination of Neuron Specific Enolase at Clinically Relevant Levels by Coupling On-line Molecularly Imprinted Solid-Phase Extraction to LC-MS/MS. Analytica Chimica Acta 2020, 1140, 210-218.

III. McKitterick N, Bicak TC, Switnicka-Plak MA, Cormack PA, Reubsaet L, Halvorsen TG. On-Line Duplex Molecularly Imprinted Solid-Phase Extraction for Analysis of Low Abundant Biomarkers in Human Serum by Liquid Chromatography-Tandem Mass Spectrometry. 2020, Submitted to Analytical Chemistry 


\section{LIST OF ABBREVIATIONS}

\begin{tabular}{|c|c|}
\hline$\overline{A A}$ & amino acid \\
\hline$A B C$ & ammonium bicarbonate \\
\hline BSA & bovine serum albumin \\
\hline CID & collision-induced dissociation \\
\hline $\mathrm{CL}$ & cross-linker \\
\hline DTT & dithiothreitol \\
\hline DVB-80 & divinylbenzene-80 \\
\hline EAMA.HCl & $\mathrm{N}$-(2-aminoethyl) methacrylamide hydrochloride \\
\hline ELISA & enzyme-linked immunosorbent assay \\
\hline $\mathrm{ESI}$ & electrospray ionization \\
\hline FM & functional monomer \\
\hline IF & imprinting factor \\
\hline $\mathrm{K}_{\mathrm{d}}$ & dissociation constant \\
\hline LC & liquid chromatography \\
\hline LC-MS/MS & liquid chromatography-tandem mass spectrometry \\
\hline LOD & limit of detection \\
\hline LOQ & limit of quantification \\
\hline$m / z$ & mass-to-charge ratio \\
\hline $\mathrm{MeCN}$ & Acetonitrile \\
\hline MIP & molecularly imprinted polymer \\
\hline
\end{tabular}




\begin{tabular}{|c|c|}
\hline MISPE & molecularly imprinted solid-phase extraction \\
\hline $\mathrm{mMIP}$ & Magnetic MIP \\
\hline MRM & multiple reaction monitoring \\
\hline MS & mass spectrometry \\
\hline NSE & neuron-specific enolase \\
\hline PP & precipitation polymerisation \\
\hline ppm & parts per million \\
\hline ProGRP & progastrin-releasing peptide \\
\hline PTM & post-translational modification \\
\hline $\mathrm{R}^{2}$ & coefficient of determination \\
\hline RIA & radiolabelled immunoassay \\
\hline $\mathrm{S} / \mathrm{N}$ & signal to noise \\
\hline SCLC & small cell lung cancer \\
\hline SDS-PAGE & sodium dodecyl sulphate-polyacrylamide gel \\
\hline SISCAPA & stable isotope standards and capture by anti-peptide antibodies \\
\hline SPE & solid-phase extraction \\
\hline SRM & selected reaction monitoring \\
\hline TCEP & tris(2-carboxyethyl)phosphine \\
\hline TFU & (trifluoromethyl)-phenyl-N' -4-vinylphenylurea \\
\hline WB & western blot \\
\hline
\end{tabular}




\section{ABSTRACT}

The aim of this thesis was the development and evaluation of novel molecularly imprinted polymers (MIPs) for the capture and analysis of small cell lung cancer (SCLC) biomarkers in human serum via bottomup proteomics. MIPs were developed in multiple of formats against the tryptic peptides NLLGLIEAK and ELPLYR, the signature peptides of progastrin releasing peptide (ProGRP) and neuron-specific enolase (NSE), respectively. Extraction protocols for the MIPs in both off- and on-line formats were carefully developed to maximise the effectiveness of the molecularly imprinted solid-phase extraction (MISPE), allowing for successful, selective capture of the target peptides. The MIPs were coupled with liquid chromatography-tandem mass spectrometry (LC-MS/MS) for quantification of the low abundant biomarkers in complex matrixes (e.g. human serum). The analytical performance of MIPs illustrates the potential for future clinical applications.

In Paper I, magnetic MIPs (mMIPs) were synthesised as core-shell and composite materials. A specific magnetic SPE protocol for MMIP sorbents for use in LC-MS/MS analysis of ProGRP was developed. Four novel mMIPs were synthesised, and the magnetic SPE method was developed and optimized for the extraction of the ProGRP signature peptide, NLLGLIEAK from human serum. The magnetic SPE was selective towards NLLGLIEAK and generated clean extracts. The magnetic SPE method demonstrated good analytical performance when coupled with LC-MS/MS; such as strong affinity towards NLLGLIEAK, acceptable recoveries (25\%), and a low limit of detection (LOD, 39 pM) and low limit of quantification (LOQ, 129 pM). The novel magnetic SPE method was comparable to other MISPE methods and demonstrated the potential for MIP-based clinical biomarker analysis.

In Paper II, MIPs targeting the signature peptide of NSE, ELPLYR were synthesised as porous polymer microspheres by precipitation polymerization (PP) conditions. The polymer microspheres were packed into trap columns for fully automated, on-line molecularly imprinted solid-phase extraction (MISPE) protocol. The on-line MISPE protocol was optimised to maximise selectivity with consideration given to the loading mobile phase, flow rate, and extraction time. The MIPs had high affinity and acceptable selectivity for ELPLYR. The MIPS successfully retained ELPLYR on-column for 40 column volumes, and the on-line MISPE method enabled $100 \%$ recovery of the biomarker over the linear range $10-100 \mathrm{ng} / \mathrm{mL}(0.25$ $2.5 \mathrm{nM}$ ) in a simple matrix. The recoveries of ELPLYR from reversed-phase SPE (RP-SPE)-treated and untreated digested human serum were $100.8 \pm 6.2 \%$ and $61.6 \pm 1.9 \%$, respectively. Extractions of ELPLYR from spiked untreated digested serum were linear in the range of $7.5-375 \mathrm{ng} / \mathrm{mL}\left(R^{2}=0.99\right)$. The LOD and 
limit of quantification LOQ for NSE in digested serum were estimated to be $1.8 \mathrm{ng} / \mathrm{mL}$ and $6 \mathrm{ng} / \mathrm{mL}$, respectively, below the median reference level of NSE in humans $(8.6 \mathrm{ng} / \mathrm{mL})$.

In Paper III, two unique MIPs were combined for automated duplex analysis of ProGRP and NSE present in human serum. Two different MIPs imprinted against the signature peptides NLLGLIEAK (ProGRP) and ELPLYR (NSE) were packed into trap columns for use in a fully automated, duplex on-line MISPE protocol. Optimisation of the on-line MISPE enhanced affinity and selectivity towards their respective signature peptide in serum. Two duplex formats were evaluated: a 1:1 w/w ratio of each polymer inside a single trap column, and two separate MIP trap columns applied in series. Both duplex formats successfully extracted the peptides from serum, however, the use of two trap columns in series gave superior peptide extraction efficiency $(85.8 \pm 3.8 \%$ and $49.1 \pm 6.7 \%$ for NLLGLIEAK and ELPLYR, respectively). The optimized protocol showed satisfactory intra-and interday precision (intraday RSD $\leq 23.4 \%$ and interday RSD $\leq 14.6 \%$ ). The duplex extraction and analysis of NSE and ProGRP spiked into digested human serum was linear $\left(R^{2}=0.99\right.$ and $\left.R^{2}=0.98\right)$ over the disease range $(0.3-30 \mathrm{nM})$ for both biomarkers. The LOD and LOQ for NSE were calculated to be $0.11 \mathrm{nM}$ and $0.37 \mathrm{nM}$, respectively, and $0.06 \mathrm{nM}$ and $0.2 \mathrm{nM}$, respectively, for ProGRP. Both biomarkers were quantified at clinically relevant levels, with the LOD for NSE being below the reference level of NSE in humans $(0.22 \mathrm{nM})$. The LOD for ProGRP was approximately eight times higher than the reference level (7.6 pM). The present work is the first reported example of an automated MIP duplex biomarker analysis. 


\section{INTRODUCTION}

The analysis of low-abundant protein biomarkers has a critical role in the clinical management of myriad diseases. The number of prospective biomarker targets grows every year; therefore, novel technologies must be developed to aid in the evaluation and analysis of prospective biomarkers. The work presented in this thesis describes the use of molecularly imprinted polymers (MIPs) as solid-phase extraction (SPE) sorbents in the extraction of signature peptides of small cell lung cancer (SCLC) biomarkers coupled with analysis by mass spectrometry (MS). Section 1.1 Molecularly Imprinted Polymers outlines and describes the history, design, synthesis, and applications of MIPs. Section 1.2 Biomarker Analysis explores protein biomarkers in the diagnosis and management of diseases and the contemporary analytical method. Section 1.3 Mass Spectrometry describes the mass spectrometer. Section 1.4 Protein Analysis by Mass Spectrometry investigates the analysis of proteins by MS by exploring proteomics, non-and targeted protein analysis, and necessary sample preparation. Finally, section 1.5 MIPs in Protein and Peptide Analysis highlights the extraction of proteins and peptides by MIPs

\subsection{Molecularly Imprinted Polymers (MIPs)}

Molecular recognition is a fundamental property of biological systems. This mechanism is involved in a range of complex, essential biological processes: such as enzymatic catalysis, antibody/antigen binding, genetic replication, transcription and translation, and biochemical receptor/ligand binding. These processes all adhere to the principle of "lock and key", first characterized by Fischer in $1894^{1}$. MIPs are synthetic polymers designed and synthesised to possess similar molecular recognition functionality ${ }^{2}$. The synthesis of MIPs is comprised of four components: The template (the imprinted molecule and target), the functional monomers (FMs, polymer units with complementary chemical properties to interact with the template), the cross-linker (CL) and the solvent. MIPs are synthesized through polymerisation around a template by FMs and cross-linked into a three-dimensional matrix. Template removal generates a polymer with selective cavities that can rebind the target molecule (Fig. 1)

This section will introduce MIPs and give a general overview of the history, design, synthesis, and formats (see section 1.5 for details on specific MIPs and their performance). 


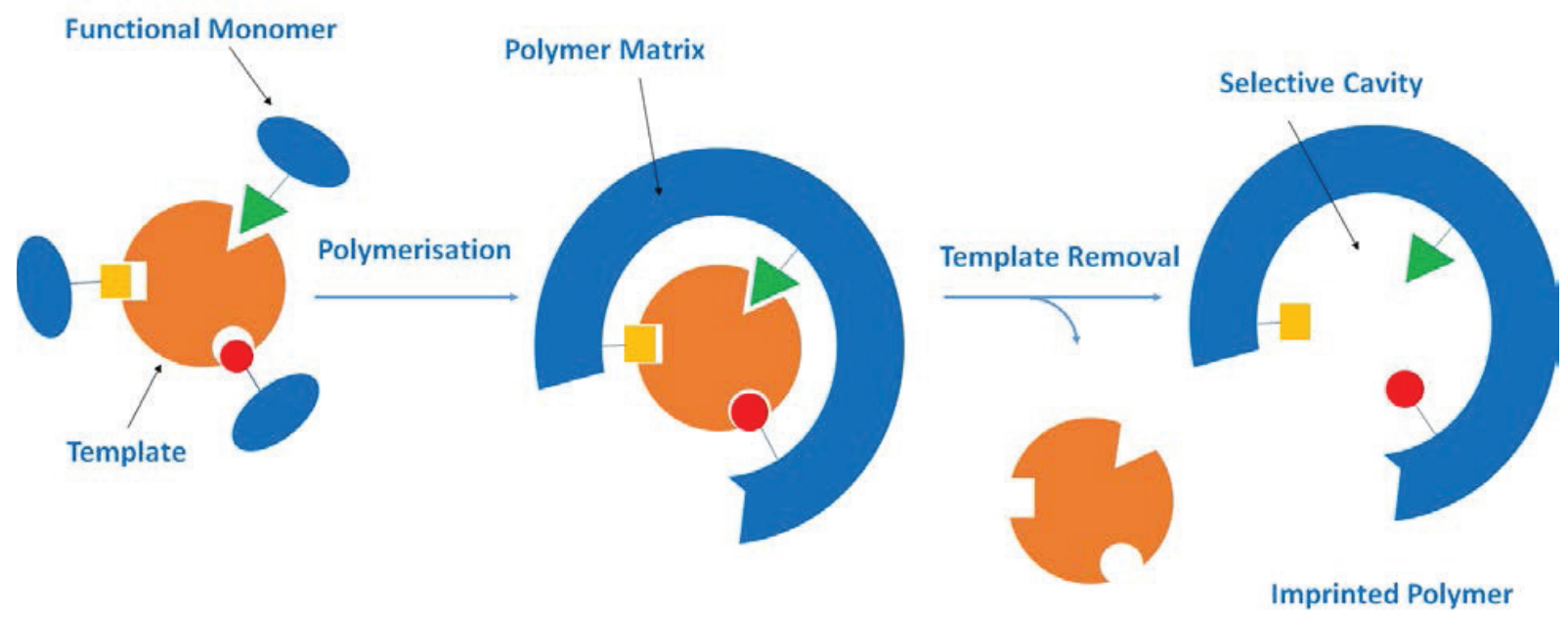

Figure 1: Schematic of MIP synthesis. A template is encapsulated by FMs and polymerisation generates a threedimensional matrix. Selective template removal generates polymer with selective cavities for target rebinding.

\subsubsection{History of MIPs}

The effects of imprinting were serendipitously discovered by Polyakov in 1931 after imprinting silica with inadvertent specificity for additives in the polymerization mixture ${ }^{3}$. The field did not see significant advancements until the first organic imprinted polymeric material (i.e. a contemporary MIP), first described in 1973 via silica particles imprinted with D-glyceric acid and D-mannitol derivatives ${ }^{4}$. This led to renewed interest in imprinting and soon the term "imprinted polymer" was coined in the early 1980 s by a group from Lund, Sweden, led by Mosbach. These imprinted materials were imprinted with heterocyclic dyes and phenylalanine analogues ${ }^{5,6}$. MIP research began to grow in earnest during the 1990 s with significant developments and broad diversification of targets, monomers, and applications ${ }^{7}$. Multidisciplinary interest in the field began to expand from polymer science into analytical and clinical chemistry as the emergence of MIPs in the use of separation science were being described in earnest. MIPs as SPE (i.e. molecularly imprinted solid-phase extraction, MISPE) materials for biomolecules ${ }^{8,9}$; and notably, the first MIPs packed into columns for automated MISPE revolutionised and modernised the field $^{10}$. However, the limited breadth of synthetic strategies initially prevented the imprinting of larger biomolecules (i.e. peptides and proteins) ${ }^{8}$. This was a particularly limiting factor as most MIP syntheses required organic solvents, which often precipitated larger, more complex proteins. Therefore, in the 1990s, the first reported MIPs targeting biomolecules were limited to simple biological molecules (e.g. amino acids, AAs). However, there were successful non-polar, organic soluble peptide and AA-imprinted MIPs reported ${ }^{10}$. The early 2000s saw the introduction of so-called "epitope imprinting" (see section 1.1.2) 
leading to an influx of peptide imprinted MIPs targeting larger biomolecules including proteins ${ }^{11,12}$. During the early 2010s, there was a greater focus on complex protein imprinted MIPs; including, whole protein imprinting, improved epitope imprinting, and single-cell imprinting (see section 1.1.2). Furthermore, the applications of MIPs diversified rapidly to include (but not limited to) SPE sorbents in chromatography ${ }^{13}$, electrochemical ${ }^{14}$ and fluorescent sensors ${ }^{15}$, drug delivery ${ }^{16}$, protein crystallization ${ }^{17}$, and catalysis ${ }^{18}$. Continuous refinements in the design and synthesis of MIPs over the last 20 years has given rise to a broad range of MIPs imprinted against macromolecular biological targets.

\subsubsection{Synthesis of MIPs for peptide and protein analysis}

Molecular imprinting has made significant progress concerning the targeting of lower molecular weight targets, producing MIPs with excellent selective recognition properties ${ }^{2}$. This has led to limited commercialization ${ }^{19}$. Presently, bio-macromolecular imprinting remains challenging, with no universal strategy to generate MIPs with selective recognition of proteins ${ }^{20}$. As such, many proteins remain difficult to imprint. This has limited the diversity of protein targets seen in the literature. The complexity of proteins remains the primary challenge concerning imprinting; namely, size, structure, physical properties, and solubility. Proteins are diverse and complex molecules that can range from $10-10,000$ $A A s^{21}$. Proteins are comprised of four orders of structure: primary (AA sequence), secondary ( $\alpha$-helices, $\beta$ sheets), tertiary (three-dimension structure), and quaternary (subunits). Exposed surface AAs can have a diverse range of properties, such as charged, polar, non-polar, or hydrophobic. The synthesis of a functional, selective MIP for protein and peptide analysis requires overcoming one or more of the structural challenges associated with proteins.

\section{Whole-protein Imprinting}

Whole protein imprinting is desirable as it can give higher adsorption capacity and selective recognition. This is due to the complexity of the binding site - unique sites with myriad binding modes, resembling classical lock and key motif. The capture of the whole protein is ideal in a complex matrix from a MISPE sample preparation standpoint; specifically, circumventing the need to modify the proteins (e.g. tryptic digest). An ideal whole protein imprinted MIP could feasibly allow for direct extraction from untreated biological samples (e.g. serum). The synthetic strategy is typically three-dimensional imprinting: the polymerization of functional monomers that encapsulate the protein. Following template removal, the resulting polymer has a three-dimensional network of specific sites. Templates for whole-protein imprinted MIPs are typically the whole, unmodified protein. 
There are several limitations with whole-protein imprinting. Firstly, the solubility of proteins in organic solvent is limited, especially for larger molecular weight proteins, restricting the total number of possible protein targets considerably. The second major limitation is the need for a large quantity of the protein for imprinting. A considerable number of proteins of clinical importance are low abundant biomarkers found in the low pM range. It would not be economically viable to extract sufficient quantities either from source or through recombinant strategies. These stark limitations are observable in the limited range of targets reported for whole-protein imprinted MIPs. High abundant and readily available bovine serum albumin (BSA), human serum albumin (HSA), hen egg-white lysozyme (HEWL) dominate the literature ${ }^{20}$. The whole-protein methodology is not viable for biomarker imprinting and other strategies must be explored when designing MIPs for use as SPE materials in the capture of proteins.

\section{Epitope Imprinting}

The epitope approach more closely resembles the antibody functionality whereby a protein epitope is imprinted. Following template removal the specific cavities function as an antibody paratope ${ }^{12}$. It must be noted that many MIPs that exploit epitope imprinting do not imprint a known epitope (i.e. a known antibody target) but rather short peptide sequences that are more suited to imprinting (e.g. linear peptide sequences). This approach circumvents the challenges associated with whole-protein imprinting. The use of a small, relatively easy to synthesize peptide as the template eliminates the need for large quantities of expensive proteins. This allows for the capture of whole proteins without the challenges of whole protein imprinting. Therefore, the number of feasible protein targets is considerably more numerous and diverse due to the exploitation of a peptide sequence suitable for imprinting (i.e. good solubility in organic solvent). Therefore, the epitope approach is much more suitable for MIPs targeting low abundant protein biomarkers. The typical peptide sequences are exposed on the protein target such as the $\mathrm{C}$ or $\mathrm{N}$ terminus or surface chains ${ }^{20}$. The use of a small section of a protein may present challenges associated with capture; specifically, the protein must interact with the binding site in the exact conformation to ensure interactions between the imprinted cavity and the imprinted epitope. As the templates are smaller than whole-proteins, there are myriad, well-established synthetic strategies to develop epitope-imprinted MIPs; including bulk polymerization ${ }^{11}$, one-pot synthesis ${ }^{22}$, and surface imprinting ${ }^{23}$. This has led to many formats (see section 1.1.3) and targets including biomarkers: beta2-microglobulin ${ }^{24}$, tumour antigen $\mathrm{p} 53^{25}$, HIV-1 antibodies ${ }^{26}$, and hepcidin ${ }^{27}$, among others. Epitope-imprinted MIPs are ideal materials in the capture of intact biomarkers. 


\section{Tryptic Peptide Imprinting}

Tryptic peptide imprinted MIPs are a variation of epitope imprinting designed to exploit the analytical power of bottom-up proteomics. Tryptic peptides allow for concurrent simplification of mass spectra and lower detection limits in MS analysis ${ }^{28}$. The capturing tryptic peptides resemble the immunoextraction technique known as stable isotope standards and capture by anti-peptide antibodies (SISCAPA) which requires anti-peptide antibodies to specifically enrich peptides to enhance detection limits ${ }^{29}$. MIPs imprinted against tryptic peptides allows for a reduction of dimensionality in protein extraction (i.e. eliminating any need for proteins to be in a specific conformation for binding). However, sample pretreatment is necessary for extraction; namely, tryptic digestion. This can be particularly challenging for low abundant proteins in complex matrices ${ }^{30}$. The direct imprinting of an unmodified tryptic peptide is not ideal as any "template bleeding" can generate an MS signal affecting the accuracy of the analysis. This is also seen in epitope and whole protein imprinting. Furthermore, due to the mechanism of tryptic digestion (see section 1.4.2) all peptides generated from trypsin will have either an arginine or lysine, except the C-terminal peptide. Therefore, if a tryptic peptide with a C-terminus arginine or lysine is imprinted, the selectivity of the resulting polymer is likely to be diminished as all tryptic peptides in a given sample have at least partial affinity towards the MIP. However, tryptic peptides can be modified before imprinting. This circumvents quantification errors usually associated with template bleeding as the target molecule in the sample will have a different $m / z$ than the modified template. Therefore, the MS will not be scanning for the template, preventing effects on the MS signal. The typical modifications to the imprinted tryptic peptides involve substituting AAs or adding functional groups on the $\mathrm{N}$-or $\mathrm{C}$-terminus. This ensures similar (but not exact) specific binding.

\subsubsection{Protein and Peptide MIP Formats}

The versatility of MIPs is observable via various formats developed to date. Each format offers unique properties suited for its specific application. The range of formats includes monolithic, nano/microparticles, magnetic particles, gels, membranes, and sensors (e.g. electrochemical and fluorescent) $)^{31}$.

\section{Monolithic MIPs}

The mechanism of MIPs (specific interactions) lends itself well in analytical chemistry, specifically in separation science. Monolithic MIPs are an attractive format for chromatography due to their ease of synthesis, permeability and, absence of any frits to contain the material (i.e. little to no polymer leakage) ${ }^{32}$. Monoliths are a continuous porous bed of hierarchical meso (i.e. $\leq 2 \mathrm{~nm}$ ) - and macroporous (i.e. $\geq 50 \mathrm{~nm}$ ) structures without the inter-particular voids seen in packed columns $s^{33}$. The monolithic structural features 
ensure excellent and easily regulated porosity throughout the structure. This ensures enhanced permeability of the monolith; therefore, stabilising flow rate, separation time, and reduces backpressures. As separation materials, monoliths have lower resistances to mass transfer kinetics, which enhances separation efficiency even at high flow rates. In addition to separation, monoliths have been used as SPE materials; particularly as on-line extraction sorbents ${ }^{34}$. However, monoliths MIP can shrink and swell in organic solvents. This can have detrimental effects on specific recognition of target analytes. Furthermore, monoliths have a lower capacity than particulate MIPs due to less surface area ${ }^{35}$. Therefore, for SPE applications, it is most common for monoliths to be crushed, sieved, washed to enhance the specific surface area ${ }^{35}$.

\section{MIP Particles}

MIP particles are quite possibly the most popular MIP format, with a significant number of MIPs being synthesised every year. MIP micro- and nanoparticles are derivatives of crushed monolithic MIPs, the smaller sizer particles (i.e. 0.1-5 $\mu \mathrm{m}$ ) give far greater surface area and allow for greater access to specific binding sites ${ }^{36}$. This ensures that the binding equilibrium is achieved at a much higher rate compared with monolithic (or crushed monolithic MIPs). The binding equilibrium is essential for SPE functionality, and particles that reach rapid equilibrium are far more desirable. Due to the mechanism of generating crushed monolith particles (i.e. imprecise grinding), the particles are often too large and polydisperse in size for effective use in SPE. MIP micro- and nanoparticles are synthesised by controlled polymerized reactions, generating small, monodisperse particles with high specific affinity for a target molecule. MIP microparticles are ideal for packing into columns for on-line SPE. However, many of the specific binding sites can be located deep into the polymer network and may be inaccessible to the target molecule or even trap the template permanently ${ }^{37}$. This could have a detrimental impact on the binding capacity of the particles. A reduced binding capacity can affect the performance of the materials during SPE, potentially affecting the analytical performance of the MIP (e.g. increased limits of detection) ${ }^{38}$. Surface imprinting micro-and nanospheres allows for the availability of high numbers of binding sites readily found on the surface of the MIP ${ }^{39}$. Another strategy to ensure polydisperse particles with binding sites close to the surface of the polymers is generating polymer core-shell materials. MIPs are imprinted around the core-shell, encapsulating it and generating a particle with uniform size distribution. Numerous MIP particles have been produced and successfully captured a range of targets; not limited to small molecules, proteins, peptides, pesticides, and drugs and their metabolites ${ }^{40}$. 


\section{Magnetic MIPs (mMIPs)}

Due to the popularity of MIP particles, many derivatives have been explored to add unique and useful functionality. One such derivative, MMIPs, involves the incorporation of magnetic properties to MIP particles. The purpose of the magnetisation of MIPs is for simplified off-line SPE of target molecules ${ }^{40}$. The use of a magnet during the SPE step allows for the target molecule to be specifically retained on the mMIP, while the matrix components and any added wash solutions can be readily removed from the sample and discarded with limited to no loss of the target analyte (provided the target analyte has sufficient binding affinity towards the MIP). In comparison, the standard off-line MIP-SPE requires many centrifugation steps to remove the matrix, while potentially increasing the risk of analyte loss through increased sample handling ${ }^{41}$. There are two typical strategies for mMIP synthesis: magnetic-core shell imprinted materials and encapsulated magnetic composite materials ${ }^{42}$. Magnetic core-shell MIPs are typically synthesised by surface polymerisation (described above) around an iron core (e.g. $\mathrm{Fe}_{3} \mathrm{O}_{4}$ ). This magnetic core-shell material has the benefit of surfaced imprinted materials (i.e. more readily accessible surface binding sites) along with magnetic properties for ease of handling ${ }^{42}$. The synthesis of encapsulated composite materials involves the magnetisation of post-imprinted materials by incorporating magnetic particles into the polymer network ${ }^{42}$. The composite nature of encapsulated mMIPs likely produces materials with inaccessible and trapping binding site ${ }^{41}$. However, the encapsulation procedure can be favourable as it allows for the magnetisation of MIPs after synthesis. This ensures that the selected MIP for magnetisation has known functionality (i.e. selectivity and specificity). This likely improves reproducibility as the imprinted step is already determined to be successful, provided the MIPs have been screened for functionality beforehand. Conversely, core-shell mMIPs require synthesis during magnetisation and therefore, MIP functionality cannot be guaranteed, and therefore, there is potential for reproducibility issues. Both formats of mMIPs in have reported as functional capture materials targeting proteins, peptides, environmental and food contaminants, etc. ${ }^{41}$

\section{Other Formats}

The versatility of MIPs allows for a vast range of diversification. As such, numerous unique formats have been designed and created. This section will briefly mention some of these in limited detail as they are out of the scope of the present work. Sensors, membranes and nanogels are unique MIP formats that exploit and combine unique functionality and molecular recognition properties of MIPs for selective capture of target molecules. Molecularly imprinted sensors are perhaps one of the most popular MIP formats. These are electrochemical, optical, fluorescent, and other sensors with imprinted polymer for selective recognition ${ }^{43}$. The detection power of the sensor in combination with selective recognition of 
MIPs allows for the quantification of targets with low detection limits at low cost with automation and miniaturisation potential ${ }^{44}$. Molecularly imprinted membranes are porous materials with imprinted sites $^{45}$. Molecularly imprinted membranes combine the diffusion process of membranes in combination with simplified, selective capture to generate clean extractions for subsequent analyses ${ }^{45}$. Nanogels are large cross-linked MIPs designed to better resemble the antibody-antigen interaction by imprinting the whole protein and retaining more of the unique three-dimensional structure of the template ${ }^{46}$.

The advancements of MIPs over the last 20 years has led to a diverse range of synthetic strategies and formats. Advancement towards MIP-based protein biomarker analysis is ongoing, with improvements and novel synthetic strategies being reported often. To understand the challenges associated with biomarker analysis, it is essential to understand contemporary biomarker analyses used in both clinical and research settings.

\subsection{Protein Biomarker Analysis}

Protein biomarker analysis is an essential aspect of the management of numerous diseases. The quantification of biomarkers is essential in the entire pathological process, from diagnosis, treatment, and disease monitoring ${ }^{47}$. Proteins as diagnostic markers have been used in pathology for nearly 200 years (the first being urinary albumin to detect kidney disease) ${ }^{47}$. Presently, the number of proteins used in clinical settings is over $200^{47}$. Historically, protein biomarker analysis has presented a consistent challenge to identify, characterise, and quantify accurately. Biological variation complicates protein analysis as a range of factors can drastically affect protein expression in the body; namely, age, sex, pathology, and drug interactions $s^{48-50}$. The complexity in both protein structure (i.e. $21 \mathrm{AAs}$, and 4 levels of structures, and post-translational modifications) and biological matrices (e.g. serum) further complicate protein analysis. Proteins in human serum have a broad dynamic range $(\mathrm{pg} / \mathrm{mL}-\mathrm{mg} / \mathrm{mL})$, and while many biomarkers are present in high concentrations, many disease markers are found in low abundance (i.e. low abundant biomarkers) requiring powerful instrumentation and excellent sample clean-up to ensure accurate analyses. The advent of immunoassays in the late $1950 \mathrm{~s}^{51}$ and electrospray ionisation (ESI) of proteins by MS in the late $1980 \mathrm{~s}^{52}$ has led to an increase in the availability of low abundant biomarkers in clinics. Despite improvements in analytical technology, novel biomarkers have very rarely been incorporated in clinical analysis in the $21^{\text {st }}$ century ${ }^{53}$. Advancements in extraction technology, multiplex approaches, and further characterisation of the human proteome will aid in discovery of novel, clinically relevant biomarkers. 


\subsubsection{Clinical Biomarker Analysis}

Clinical biomarker analysis is the identification and quantification of one or more biologically relevant endogenous compounds in a biological sample for diagnosis and monitoring of human disease ${ }^{54}$. A protein is defined as a biomarker only when it is related to a specific biological function or disease state (e.g. the aforementioned urinary albumin in kidney disease). Rapid, reliable, and robust clinical biomarker analysis is essential in modern medicine. Biomarkers can be drawn from numerous sources such as tissues, blood and other biofluids. Blood-borne biomarkers are more desirable targets as identification and quantification can be performed in hours while only requiring a patient to undergo venepuncture (i.e. a blood test), a non-invasive procedure. Many aggressive diseases are reliant on rapid diagnosis for better prognoses, such as SCLC. Elevated levels of the biomarkers progastrin-releasing peptide (ProGRP) and neuron-specific enolase (NSE) is a clear indicator of SCLC ${ }^{55}$. Before the routine analysis of these biomarkers, the diagnosis required an invasive lung biopsy, often occurring in the late stages of the disease. Presently, many diseases do not have routine biomarkers available for clinicians and pathologists. More work is needed to translate biomarker discovery into routine clinical analysis ${ }^{56}$. At present the gold standard in clinical biomarker analysis of proteins is immunoassays. These assays have been a mainstay of pathology for decades. Section 1.2.2 will provide insights into immunoassays, including key advantages and limitations.

\subsubsection{Immunoassays}

Immunoassays remain the preferred method for protein analysis in clinical settings due to their ease of use, automation, specificity, and excellent detection limits ${ }^{40}$. Immunoassays are so named due to the use of antibodies for specific capture of an analyte (i.e. an antigen) and utilised a range of highly sensitive detection systems from radiolabelling, fluorescence, and chemiluminescence ${ }^{57}$. The three most common forms of immunoassays are radiolabelled immunoassay (RIA), enzyme-linked immunosorbent assay (ELISA), and western blotting ${ }^{57}$. RIA is the oldest immunoassay technique used in clinics. It is a straightforward procedure: a known concentration of labelled antigen (i.e. a reporter antigen) is mixed with an unknown concentration of unlabelled antigen (i.e. in the patient sample). Both antigens compete for the antibody paratope (i.e. antibody binding site) and by measuring the displacement of the radiolabelled antigen, the amount of unlabelled antigen can be back-calculated ${ }^{58}$.

Western blot (WB) assays target specific proteins from complex matrices for both identification and quantification ${ }^{59}$. WB is less versatile compared to ELISA and RIA and, in clinical settings, are used for conformation analysis of certain ELISA assays. WB is more hands-on compared to ELISA with three 
separate manual preparation stages: protein by separation gel electrophoresis, transfer to a nylon membrane, followed by antibody capture and signal measurement ${ }^{59}$. Separation before the addition of antibodies gives WB higher specificity and sensitivity with respect to ELISA without the cross-reactivity and specificity challenges of some ELISA assays ${ }^{59}$ (see section 1.2.4).

\subsubsection{Enzyme-linked Immunosorbent Assay}

ELISA, first described in 1972 by Engvall and Perlmann ${ }^{60}$ vastly simplified the immunoassay and rapidly became the clinical gold standard. ELISA measures the response generated by functionalised antibodies, which targets specific antigens in a patient sample ${ }^{61}$. By measuring the antibodies that directly target a compound, the assay has enhanced sensitivity compared with other immunoassays, furthermore, the conjugate enzyme functionality (e.g. fluorescence, chemiluminescence, etc.) of the antibodies allow for more user-friendly and cheaper measurements; thereby, simultaneously improving assay performance, cost, and throughput. ELISA assays can be divided into two classifications: homogenous and heterogenous ${ }^{62}$. Homogenous ELISAs are less commonly used in clinics due to their cost and poorer selectivity. The more common assay, particularly for protein analysis are heterogeneous assays ${ }^{62}$. Heterogeneous assays involve immobilisation to solid supports. This allows for the target antigen to be fixed as other matrix components are washed away. This not only simplifies that sample but additionally removes any free unbound target analyte, reducing the false positive rate ${ }^{63}$. This enhances the accuracy and specificity of the assay, particularly in biological samples. Improvements over the close to five decades have yielded four clinically relevant subtypes of ELISA assay (Fig. 2); namely, direct, indirect, sandwich, and competitive ELISA assays ${ }^{64}$.

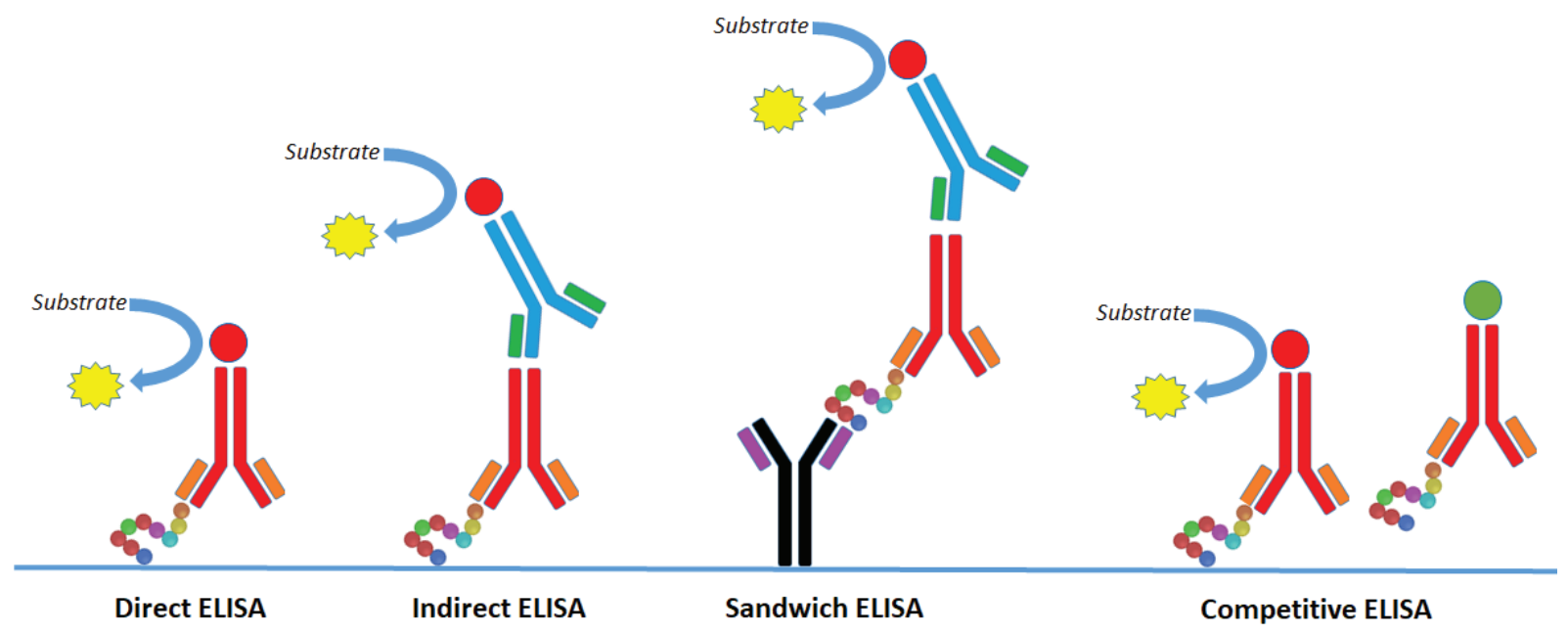

Figure 2: The four clinically relevant formats of ELISA assays: direct, indirect, sandwich, and competitive ELISA. Biomarkers are quantified by adding a substrate which reacts with an enzyme-linked to either the capture or reporter antibody. This generates a quantifiable signal. 
Direct ELISA is the simplest sub-type, where patient samples containing the target antigen are fixed (typically to the walls of a microplate). An enzyme-conjugated antibody specific for the antigen is added to the sample, binding to the immobilised target. Addition of a substrate for the conjugated enzyme induces an enzymatic reaction, generating a signal for quantitative measurement (e.g. fluorescence) ${ }^{65}$. Direct ELISA is easy to use and requires fewer reagents and has no possibility for cross-reactivity but is less sensitive than the other ELISA formats ${ }^{66}$. Indirect ELISA has similar sample preparation to direct ELISA (e.g. sample adherence to plate walls, blocking buffers added). A primary, target antigen-specific antibody is again added to the sample. This is followed by the addition of a secondary reporter antibody containing a conjugate enzyme is added. Enzyme substrate reacts with the secondary antibody-conjugated enzyme to generate a quantifiable signal ${ }^{64}$. Sandwich ELISA is a more antigen-specific technique. Known amounts of antigen-specific antibodies are immobilised on the plate walls instead of the sample. Another antigenspecific antibody is added to bind and "sandwiches" the target antigen. Enzyme-conjugated secondary antibodies, specific for the primary antibody are added which react with the added substrate to generate the quantifiable signal ${ }^{64}$. Competitive ELISA features a competitive reaction between the sample antigen and added antigen (bound to the plate) with the primary antibody. Primary antibodies are added to the patient sample generating antibody-antigen complexes. These complexes are added to plates pre-coated with the same antigen. Following incubation, the remaining unbound antibody is washed and discarded. A secondary antibody with a conjugated enzyme is added, followed by enzyme-substrate to generate the signal ${ }^{64}$. The lack of signal indicates the presence of antigen in the sample ${ }^{67}$.

The role of ELISA in clinics cannot be overstated. Presently, over 100 proteins and 60 peptides are analysed by FDA approved ELISA methods ${ }^{68}$. Commercial ELISA kits are a widely available and growing market. The market size was estimated to be USD 18.02 billion in $2018^{69}$ and will doubtless increase as tests for COVID19 antibodies will be in very high demand in the years to come. ELISA while indispensable in modern clinics, for details on immunoassay limitations, see section 1.2.4.

\subsubsection{Immunoassay Limitations}

Immunoassays are the gold standard in clinical biomarker analysis but despite their ubiquity, several key limitations persist despite over more than half a century of refinements. As immunoassays require highquality antibodies, it is essential commercially available kits are reliable. High variation in commercial kit accuracy and reliability have been shown. Any variation in reliability could lead to false reporting, possibly affecting diagnoses and patient outcomes. This variation is especially seen in the production of antibodies 
for novel biomarker discovery targets. This likely a contributing factor in the lack of biomarkers being added in routine clinical analysis.

Singleplex immunoassays (i.e. an assay with only one target) are the primary clinical format used at present ${ }^{70}$. While many diseases can rely on single analyte analysis for optimal patient outcomes (e.g. HIV$1 \mathrm{p} 24)^{40}$, it has become more apparent that many diseases necessitate the analyses of multiple biomarkers for optimal disease management. One notable disease is SCLC, with ProGRP and NSE levels monitored together for accurate diagnoses and treatment $\mathrm{s}^{55}$. Two separate singleplex assays are more laborious, expensive, and require significant quantities of patient sample ${ }^{71}$. The development of multiplex immunoassays has been slow but some assays have been brought to the market ${ }^{72}$. There are considerable advantages of multiplex assays: reduced labour and consumable cost, lower sample volumes, lower turnaround times, and improved disease monitoring ${ }^{72}$. Broader biomarker information allows for a larger focus on patient-specific treatments ${ }^{40,72}$. Despite the broad use of immunoassays, there are still some key limitations. Perhaps the most significant, persistent issue with immunoassays, both single- and multiplex, is cross-reactivity ${ }^{73}$. Any use of reporter antibodies will have the possibility of cross-reactivity, potentially perturbing assay performance. This typically results in an increase in detection limits (i.e. due to higher background noise) an increased incidence of false positives or negatives ${ }^{40,73}$. 


\subsection{Liquid Chromatography-Mass Spectrometry (LC-MS)}

LC-MS is a powerful technique that has been indispensable in analytical chemistry for decades. Mass spectrometers are powerful instruments that allow for the detection and quantification of analytes in the attomolar $\left(10^{-18}\right)$ range $^{74}$. MS analysis occurs in the gas phase using pre-ionized analytes. All mass spectrometers are comprised of an ion source, the mass analyser, detector, and processing software. The ion source ionises analytes, the mass analyser measures the mass-to-charge ratio $(\mathrm{m} / \mathrm{z})$ of the ionized analytes. The detector registers and/or quantifies ions' $m / z$ values. Finally, the software allows for complex data processing to generate a mass spectrum and in tandem MS (i.e. MS/MS), a chromatogram ${ }^{75}$. The present work used liquid chromatography-tandem mass spectrometry (LC-MS/MS) extensively for data generation. Therefore, the following section will describe the important aspects of LC-MS/MS

\subsubsection{Liquid Chromatography}

Liquid chromatography (LC) separation plays an essential role in protein analysis by MS. LC systems are widely preferred to gel-based separation methods for the separation of proteins and peptides. This is due to the versatility and diverse range of stationary and mobile phases along with high reproducibility and compatibility with electrospray-based MS systems. Reversed-phase (RP) stationary phases are the most common in MS-based protein analysis due to the mobile phases having excellent compatibility with electrospray ionisation (ESI). The mobile phase in RP chromatography consists of water and a watermiscible organic solvent mixture. Additives are added to the mobile phase to alter the charges of proteins or peptides to improve ionisation (e.g. formic acid, FA for positive MS). Enhanced resolution generated by LC separation significantly improves MS signals. High-resolution separation of components significantly reduces co-elution of undesirable species along with the target analytes. This can greatly reduce ion suppression and background noise in MS.

\subsubsection{Ion Sources and lonisation}

MS is dependent on the ionisation of analytes for analysis. There are a range of ionisation techniques depending on the nature of the target analytes and the matrix being ionised. ESI and matrix-assisted laser desorption/ionization (MALDI) are the two techniques most commonly used to ionise the proteins or peptides for mass spectrometric analysis since the late $1980 \mathrm{~s}^{52,76}$. ESI takes analytes in solution and induces ionisation before the analytes enter the mass analyser, as such, it can be readily coupled to liquidbased separation systems (e.g. HPLC, electrophoresis) ${ }^{76}$. ESI is a soft ionization technique, ensuring little to no fragmentation of the target species. This preserves the structure of the target analytes and allows for greater control when the analytes enter the mass analysers ${ }^{77}$. ESI haves three key stages: dispersal of 
charge droplets, solvent evaporation, and ejection of ions from the charged droplets ${ }^{78}$. The application capillary voltage creates a mist of highly charged droplets with the chosen polarity. Nebulising gas (e.g. nitrogen), helps to convert the solution to a gaseous phase. Charged droplets are generated at the electrospray tip, a potential gradient directs the electrospray toward the analyser region of the mass spectrometer (Fig. 3). The emitted gaseous ions are further directed by a skimmer cone and accelerated into the mass analyser for analysis of $\mathrm{m} / \mathrm{z}$ and intensity ${ }^{79}$. ESI is efficient, straightforward, and can ionise myriad analytes of interest in complex samples. As such, it is immensely popular in modern laboratories.

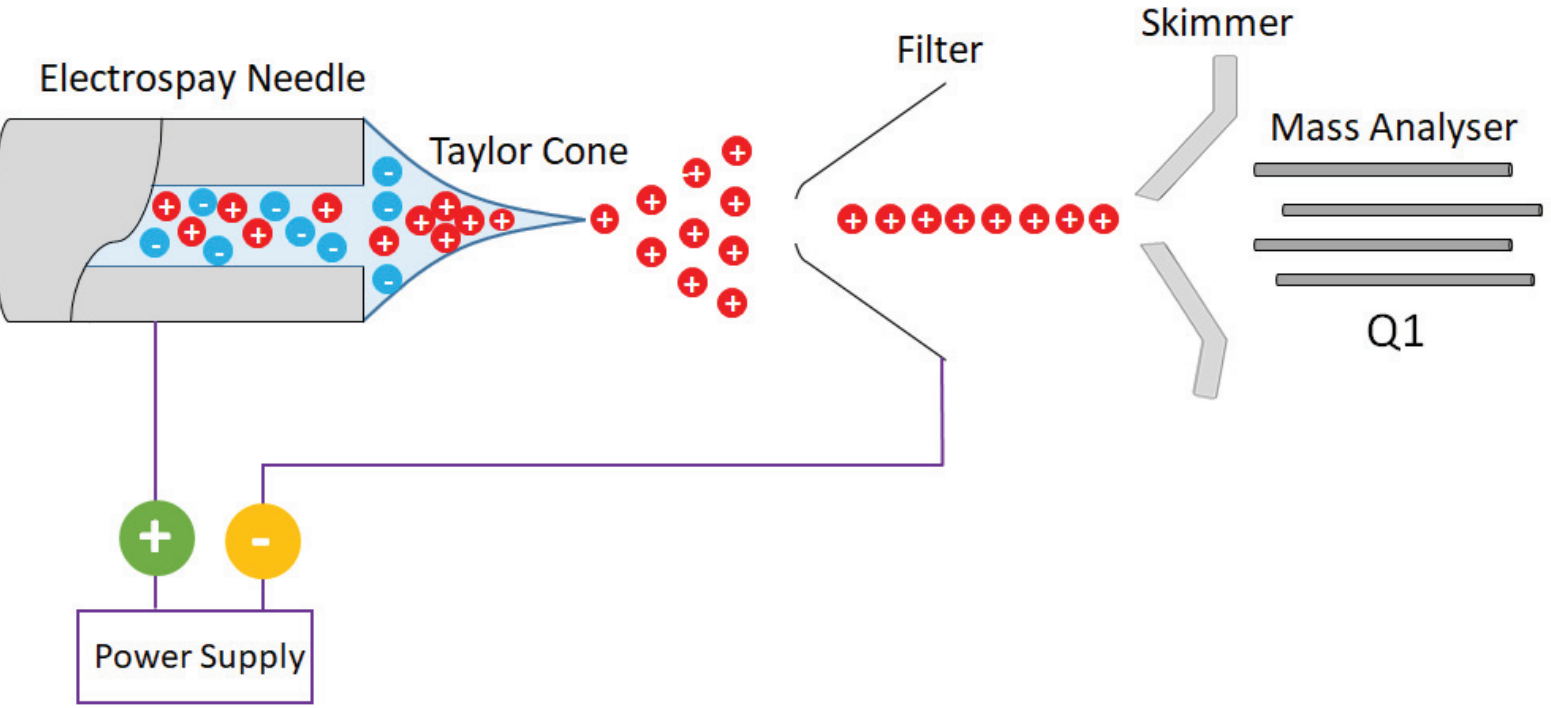

Figure 3: Electrospray Ionisation coupled to a quadrupole mass analyser. The liquid sample is passed through the electrospray needle which is charged with a power supply. This generates a charge which ionises the liquid sample, forming charged species. These are then directed towards the filter cone. The charged species pass through a skimmer into the mass analyser for measurement and analysis.

MALDI is perhaps the next most popular ionisation technique in protein and peptide analysis by MS. MALDI incorporates sublimation and subsequent ionisation of dry, crystalline matrix samples via laser pulses. MALDI is also a soft ionization technique using lasers directed towards a matrix to ionise the samples without fragmentation ${ }^{80}$. Larger molecular weight proteins are often not fully ionisable by MALDI and often fragment or breakdown. MALDI-MS requires relatively pure samples of simple peptide mixtures, whereas ESI-MS systems are often preferred for complex sample analyses ${ }^{80}$. 


\subsubsection{Quadrupole Mass Analysers}

Irrespective of the ionisation technique, the mass analyser is the core of the MS and is responsible for the separation of species based on their $\mathrm{m} / \mathrm{z}$ ratios ${ }^{81}$. There are many mass analysers, each with unique systems for ion separation. The present work utilised quadrupole MS to generate the vast majority of data. As such, the following section will highlight the functionality of quadrupole and triple quadrupole mass analysers.

A quadrupole consists of four parallel electrical rods with two that apply direct current (DC) and two that use alternating radiofrequency $(\mathrm{rf})^{81}$. Ions are generated in an ionization chamber which is pulsed toward the quadrupole by an electrical field. Positively charged ions are directed towards the negatively charged $\operatorname{rod}^{81}$. However, if the polarity of the electrical field is reversed, the ions will alter their trajectory before striking the rod. Therefore, ions have an oscillatory path and by controlling the voltage and frequency, ions in a narrow $\mathrm{m} / \mathrm{z}$ range will be able to pass through the quadrupole without striking the rods and reach the detector. This allows for the selection of the ions of interest ${ }^{81}$.

Quadrupole analysers are cheap, small, and easy to maintain. A quadrupole has a very narrow mass range ( $\sim 000 \mathrm{~m} / \mathrm{z}$ ) and low resolution. However, quadrupoles can be combined with other mass analysers for MS/MS analysis such as triple quadrupole, quadrupole time-of-flight (qTOF) and as ion guides in highresolution instruments ${ }^{81}$.

Perhaps the most common MS/MS instrument is the triple quadrupole, where two quadrupoles and a collision cell (often a hexapole) are connected in series ${ }^{82}$. These quadrupoles can be used in a variety of scanning modes. Most pertinent to the present work and perhaps most common is selected reaction monitoring (SRM) or, multiple reaction monitoring (MRM) for multiple ions per analysis (Fig. 4) ${ }^{82}$. Selected ion/s for MS/MS analysis are filtered by the first quadrupole (Q1). The second quadrupole acts as a collision cell and applies inert gas (e.g. helium, nitrogen, or argon) ${ }^{83}$. Collision energy can be controlled to generate specific fragments of interest. This process is called collision-induced dissociation (CID $)^{84}$. The third quadrupole (Q3) can filter out the fragments and send them to the detector. SRM and MRM can be 
used to generate a mass spectrum and chromatogram of only desired ions and is excellent in the quantification of known species in complex matrices ${ }^{82}$.

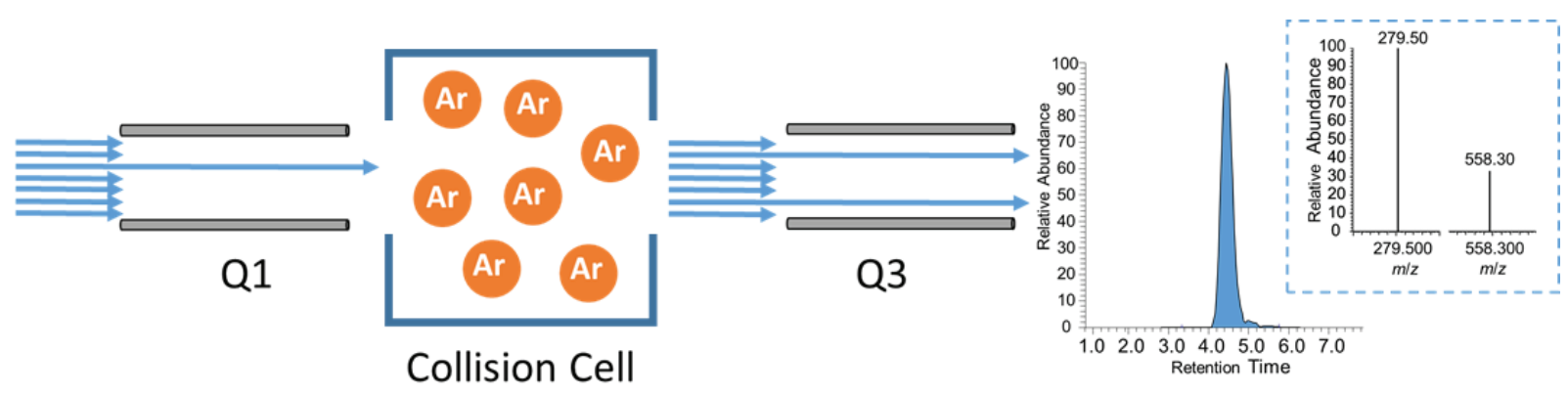

Figure 4: MRM using a triple quadrupole mass spectrometer. The first quadrupole is used to separate the parent ion of the desired species. The collision cell (often a hexapole) is filled with a collision gas (e.g. argon) which breaks up the parent ion into fragment ions. These fragment ions are filtered by the $3^{\text {rd }}$ quadrupole before measurement by the detector. In combination with LC-MS/MS, a triple quadrupole generates a chromatogram and mass spectrum.

\subsection{Protein Analysis by Mass Spectrometry}

The limitations in standard immunoassays outlined in section 1.2; namely, cross-reactivity and limited multiplex capabilities have led to a search for alternative protein analysis techniques ${ }^{85}$. As such, interest in MS-based proteomics and protein analysis has been increasing over the last two decades ${ }^{86}$. MS has been extraordinarily successful in the quantitation and/or qualification of proteins and peptides. There are three strategies for protein analysis by MS: top-down, bottom-up, and middle-down protein analysis.

\subsubsection{Top-and middle-down Protein Analysis}

Top-down proteomics is the measurement of intact proteins by MS. Naturally, this generates key information on the entire proteome including post-translation modifications (PTMs), mutations, and isoforms. Top-down approaches allow for the identification of any changes in protein structure from primary to quaternary ${ }^{87}$. Despite the excellent information generated, the analysis of whole proteins is challenging in both the chromatographic separation and MS analysis. Whole proteins are large and can have poor diffusion during LC separation ${ }^{88}$. This limits analysis of more complex samples as poor chromatographic separation will likely lead to matrix effects (e.g. ion suppression) and high background ${ }^{88}$. For MS analysis, the top-down approach is typically reserved for high-end, high-resolution instruments that have sensitivity, resolution, and high-quality fragmentation ${ }^{89}$. High sensitivity is essential due to the complexity of the generated mass spectrum. ESI of many proteins causes a wide distribution of charge states. With numerous charge states, the signal of a protein can easily be split into hundreds of peaks ${ }^{90}$. This leads to a dilution of signal for each $\mathrm{m} / \mathrm{z}$ ratio. Therefore, sensitivity is often compromised beyond 
the capabilities of low-end MS systems. Likewise, high resolution and mass accuracy are essential in the separation and accurate assignment of spectral peaks from highly complex precursor spectra that contains numerous intact proteoforms, or fragmentation spectra with many hundred fragment ions ${ }^{90}$. Furthermore, fragmentation (essential for quantification) is challenging with many collision-induced dissociation (CID) systems, not being powerful enough ${ }^{91}$. Despite the technical challenges, top-down proteomics has successfully identified numerous novel proteins and proteoforms ${ }^{91}$.

Middle-down MS is often described as a compromise between bottom-up and top-down proteomics ${ }^{92}$. Middle-down was developed to analyse long polypeptides that still retain more information about the proteoform compared to bottom-up MS, but without many of the technical challenges of top-down $\mathrm{MS}^{93}$. Middle-down MS incorporates proteases into the sample preparation to generate polypeptide chains that still retain key structural information. This somewhat circumvents the sensitivity challenges with topdown MS, as there are significantly fewer charge states and therefore, less complex spectra. Middle-down is used less compared to top-down or bottom-up but has seen success in histone PTM analysis. ${ }^{92,94}$

While top- and middle-down proteomics have had success in the identification of proteins and their proteoforms, the bottom-up approach remains the more preferred technique and has been a mainstay in proteomics for decades; particularly in protein and peptide identification ${ }^{28}$.

\subsubsection{Bottom-up Protein Analysis}

Bottom-up protein analysis is the identification and/or quantification of proteins by analysis of peptides generated via proteolysis ${ }^{28}$. Bottom-up protein analysis allows for the indirect characterisation of proteins via the peptides produced following proteolytic digestion using a protease (e.g. trypsin). Peptides can be identified with theoretical MS/MS spectra generated from in silico digestion of a protein database ${ }^{28}$. For quantification, particularly in complex mixtures, the use of a signature peptide is essential. A signature peptide is a proteolytic peptide that is unique to a single protein. The cleavage of a protein into small peptides increase the complexity of the mass spectra since a protein gives on average rise to 50 proteolytic peptides. However, good LC separation of peptides is less challenging compared to proteins. The result of this is higher sensitivity and simplified analyses ${ }^{95}$. As the sensitivity is increased, high-resolution instruments are not essential and triple quadrupole and other lower-resolution instruments are viable in the quantification of proteins ${ }^{95}$. However, close to complete digestion is essential in bottom-up proteomics and the proteolysis of proteins is not always straightforward. The sample preparation contains many steps and is a more time-consuming approach, less suited for high-throughput analyses. Proteolytic digestion is outlined below. 


\section{Reduction and Alkylation}

The tertiary structure (i.e. disulfide bridges) function to stabilize proteins. However, for effective proteolysis, these intra-molecular bonds must be broken as these 3D structures can hinder enzymatic access to cleavage sites, leading to poor digestion quality (i.e. fewer tryptic peptides or missed cleavages). Any protein that has two cysteine groups can form disulfide bonds due to the thiol groups. Reduction reactions are used to break these bonds and provide access to a linear AA sequence. Various reduction agents are used but the most common are tris-2(-carboxyethyl)-phosphine (TCEP) and dithiothreitol $(\mathrm{DTT})^{96}$. DTT has a much lower redox potential than other common reducing agents (e.g. $\beta$ mercaptoethanol, therefore, DTT has the same reduction strength at lower concentrations ${ }^{97}$. DTT first reduces disulfide bonds before two sequential thiol-disulfide exchange reactions occur. This results in thiol groups of the cysteine having free $\mathrm{SH}$-groups ${ }^{98}$. The reduction reaction is a reversible process and requires a follow-up alkylation reaction to prevent the reformation of the disulfide bonds. Alkylation is performed by alkylating agents such as iodoacetic acid (IAC) or iodoacetamide (IAA) ${ }^{99}$. These agents alkylate the free SH-groups, thereby, preventing cysteine-cysteine interactions and allowing for easier enzymatic digestion ${ }^{99}$

\section{Digestion}

Proteolysis for bottom-up proteomics is a controlled process, with proteases selected based on the desired proteotypic peptides ${ }^{100}$. This is essential in targeted proteomics to generate the signature peptide of interest. There are a variety of proteases, each targeting specific AAs of the primary protein structure ${ }^{100}$. By far the most common is the mammalian digestive enzyme, trypsin ${ }^{100}$. Trypsin is produced in the pancreas in many mammals and can be purified in high volumes cheaply. Trypsin specifically hydrolyses after arginine and lysine AAs if not followed by a proline ${ }^{101}$. Trypsin is often added to reduced and alkylated proteins at $37^{\circ} \mathrm{C}$ from $2-24$ hours. The optimal pH for the enzymatic activity of trypsin is between 7.5 and 8.5 (i.e. the $\mathrm{pH}$ of many biological fluids such as serum) ${ }^{102}$. Therefore, trypsin has excellent compatibility with biological samples.

\subsubsection{Non-Targeted Bottom-up Protein Analysis}

Bottom-up analyses are divided into targeted and non-targeted analyses. Non-targeted protein analyses are typically discovery experiments whereby biological samples such as cell lysates or serum are analysed without a specific target. The primary goal of the analysis is to determine which proteins are present in a given sample or protein discovery (i.e. biomarker discovery). Due to the complexity of the spectra of nontargeted analyses, high-resolution instruments are required (e.g. orbitrap- or TOF-mass analysers). These instruments can generate mass spectra for 1000s of proteins and peptides using data-dependent 
acquisition (DDA) and in more recent time data-independent acquisition (DIA). In DDA the ions with the most intense signal are selected by the instrument for fragmentation and MS/MS analysis. Proteomics software uses calculations and databases to interoperate the mass spectra and uses the $m / z$ values of the selected species' precursor ions (i.e. from MS1) the fragmentation pattern (i.e. from MS2) to determine the AA sequence of the analysed species and determine the protein which generated the peptide.

\subsubsection{Targeted Bottom-up Protein Analysis}

Targeted MS protein analysis is used when the protein of interest is known. Typically, targeted MS analysis is performed by the SRM and MRM by triple quadrupole of a signature peptide. This due to the specificity of SRM and MRM as described in section 1.3.3. A targeted approach is used in protein quantification, where a known protein of interest is quantified from a signature peptide. While SRM and MRM are specific methods, the variance is ESI; namely, spray stability, matrix effects (i.e. ion suppression by matrix components), and losses during sample clean-up, must be accounted for to ensure accurate quantification. To this end, a known concentration of the internal standard (IS) is added to samples during sample preparation or immediately before analysis. The ideal IS in the quantitative bottom-up analysis is a heavy isotope-labelled synthetic variant of the target peptide. Typically, the C-terminal AA (i.e. arginine or lysine) is exchanged with a heavy isotope labelled AAs containing ${ }^{13} \mathrm{C}$ and ${ }^{15} \mathrm{~N}$. A labelled target peptide is more ideal as it will behave the same during sample preparation as the native target peptide in MS analysis. However, the MS will easily distinguish the IS from the target peptide as the $m / z$ values are slightly increased due to the heavy isotopes. By measuring the IS/target peptide ratio, the effects of sample loss during sample preparation and ESI variability can be accounted for and accurate quantification of tryptic peptides is possible in complex samples, including patient samples.

\subsubsection{Sample Clean-up}

Sample clean-up is an essential aspect of quantitative protein analysis and is presently a significant challenge. This is particularly the case when quantifying low-abundant proteins from complex samples (e.g. serum). This is due to the dynamic range of the various matrix components of biological samples. In human serum there are 10 orders of magnitude in protein concentrations from $\approx 50 \mathrm{mg} / \mathrm{mL}$ to $\approx 5 \mathrm{pg} / \mathrm{mL}^{103}$ with $\geq 99 \%$ of total protein belong to the few highest abundant species (e.g. serum albumin). The overwhelming concentrations of non-specific (i.e. non-targeted species) tryptic peptides generated in a digest of human serum can easily perturb the ESI process by matrix effects (e.g. ion suppression). Additionally, ESI-MS is a sensitive instrument and high concentrations (e.g. $\mathrm{mg} / \mathrm{mL}$ ) can affect the 
functionality of the system or potentially clog the plumbing. These issues can lead to severe losses in the sensitivity of the instrument or prevent analysis altogether. Therefore, an effective sample clean-up must be performed in most cases.

Naturally, the primary goal of sample clean-up is to generate the sufficiently cleanest sample possible and there are many strategies to extract the target protein or peptide of interest. These include electrophoresis (i.e. separation based on isoelectric point and molecular weight), solid-phase extraction (SPE, i.e. separation based on chemical properties), matrix depletion (removing a majority of the matrix components), and immunoaffinity methods (described in section 1.4.6).

Matrix depletion involves the removal of many matrix components. The cheapest and easiest is matrix precipitation. This involves the addition of a precipitating agent (e.g. organic solvent and acid) that forces high-abundant matrix components to become insoluble. This allows for the removal of these high abundant species by simple centrifugation. This is particularly effective for proteins such as albumin and immunoglobulins. Precipitation cannot be used for every protein of interest. If the target protein is large (i.e. $\geq 50 \mathrm{kDa}$ ), it is more like to precipitate with the rest of the high-abundant species. More targeted approaches are often used to reduce sample loss as much as possible. Immunoaffinity extraction is commonly used with antibodies targeting the most common high abundance proteins used alone or in combination.

Sodium dodecyl sulphate-polyacrylamide gel (SDS-PAGE) is a targeted technique that separates proteins in two-dimensions based on their size and isoelectric point. This generates a ladder of separate proteins based on molecular weight. As the molecular weight of the target protein is known, its section of the gel can be cut out. In-gel digestion is performed and the peptides are extracted from the gel before analysis. While this is often effective in extracting the target protein, it is laborious, has poor reproducibility, and exceptionally low throughput. As such, it is less preferred than matrix depletion or SPE strategies.

SPE is a widely used sample clean up method for desalting tryptic peptide samples following digestion sample preparation. Uncommonly, SPE can be used to extract peptides of interest; particularly if the SPE has specific functionality. These properties (e.g. polarity, hydrophobicity, charge) can be exploited by suspensions of solid, functionalised particles as a stationary phase. The functionalisation and particles available are diverse. SPE involves several steps, firstly the sample is passed through the stationary phase, and the target peptides are retained. The stationary phase is then washed to remove any weakly bound matrix components. Finally, the target peptides are eluted off the station phase. SPE has seen widespread 
use in sample clean-up for MS analysis. This is due to functional diversity, ease of use, and high throughput. Many materials are packed into columns for use in automated SPE, further improving throughput. As described in section 1.1.3, MIPs are ideal for SPE materials and have been used extensively in sample-clean up.

\subsubsection{Immunoaffinity and Mass Spectrometry}

Antibodies can be excellent tools in sample clean-up and can be used in tandem with MS. Cross-reactivity among antibodies is near-ubiquitous and presents a significant challenge to overcome ${ }^{104}$. MS has gained more interest over the last few decades in protein analysis to circumvent cross-reactivity and limited multiplex capabilities of immunoassays. MS allows for highly selective, sensitive, and accurate analyses of multiple proteins and peptides without cross-reactivity (see section 1.3 for details on MS function and analyses). However, to determine low abundance proteins a selective sample clean-up step is necessary. ${ }^{105}$ The use of immunoaffinity clean-up in combination with MS/MS has shown to have greater differential power than conventional immunoassays while circumventing the limitations of crossreactivity, and improving the detection limits of mass spectrometry ${ }^{106}$. Single immobilised antibodies have successfully quantified multiple protein isoforms from a single sample ${ }^{107-109}$ as well as protein cleavage products ${ }^{110,111}$. Furthermore, combinations of immobilized antibodies have been used to quantify multiple protein biomarkers from a single sample, including, multiplex analyses of the SCLC biomarkers, ProGRP, and NSE in patient samples ${ }^{112-115}$. 


\subsection{MIPs in Protein and Peptide Analysis}

MIPs have been used in the capture and analysis of proteins and peptides since the late 1990s. As previously described, MIPs are widely diverse materials with an array of formats. Many of these formats are imprinted with protein, peptide, or epitopes for specific capture. The following section will describe the performance of the various MIP formats in protein and peptide extraction and analysis. Some of the MIPs listed in this section will be highlighted to provide a general overview of the trends in MIP extractions of proteins and peptides.

\subsubsection{Molecularly Imprinted SPE (MISPE)}

As described in section 1.1, MIPs used in MISPE are typically micro- and nanoporous particle materials. This is due to their versatility as extraction sorbents in both on-and off-line MISPE. These SPE materials can be combined with analytical instrumentation for the analysis and quantification of proteins. Many of these particles are whole-protein imprinted materials designed to capture whole proteins as seen in Table 1. The target proteins range from high molecular weight (e.g. glucose oxidase ${ }^{116}, 145 \mathrm{kDa}$ ) to low molecular weight peptides (e.g. Angiotensin II17, 1 kDa). The glucose oxidase MIPs were an early example of a whole-protein imprinted MIP for use in off-line MISPE. This MIP successfully captured glucose oxidase from a simple matrix ${ }^{116}$. A few years later, MIPs imprinted with modified AAs were developed to exploit specific AA functionality ${ }^{118}$. These MIPs demonstrated excellent binding affinities and demonstrated the potential of exploiting specific AAs to retain peptides. This concept was developed further with epitope imprinted MIPs ${ }^{119}$. The MIPs were designed to exploit specific epitopes of the target pepB-type natriuretic peptide (BiP).

Epitope imprinted MIPs are particularly common in MIPs for use in on-line MISPE. Targets include ProGRP, angiotensin II, beta2-microglobulin (B2M), and oxytocin. These MIPs allow for the automated extraction of proteins and peptides. The B2M MIP was imprinted with the epitope MIQRTPKIQ and used for the capture of the whole proteins from human serum and analysis by HPLC-UV ${ }^{24}$. The on-line MISPE protocol had good analytical performance with detection limits well below the reference levels in humans.

Another imprinting strategy seen in particle MIPs is the use of tryptic peptides for the capture of signature peptides. A MIP imprinted with modified NLLGLIEAK (to prevent template bleeding) was developed for bottom-up quantification by LC-MS/MS of the low-abundant biomarker ProGRP ${ }^{120}$. The on-line MISPE extraction protocol allowed for the quantification of ProGRP in patient samples. MISPE materials remain popular and many are being developed each year for novel protein and peptide targets. 
Table 1: MIPs for the extraction of proteins and peptides by MISPE

\begin{tabular}{|c|c|c|c|}
\hline Target & Molecule & Template & SPE format \\
\hline Glucose Oxidase $^{116}$ & Protein & Glucose oxidase & Off-line MISPE \\
\hline BNP $^{119}$ & Peptide & EVATEGIR/LQESPRPTG & Off-line MISPE \\
\hline Sulfated peptide $\mathrm{e}^{121}$ & Peptide & Fmoc-pTyr-OEt & Off-line MISPE \\
\hline Histidine peptides ${ }^{118}$ & Peptide & Histidine & Off-line MISPE \\
\hline Pepsin ${ }^{122}$ & Protein & Pepsin & Off-line MISPE \\
\hline $\mathrm{HMWK}^{123}$ & Protein & DQGHGHQ & Off-line MISPE \\
\hline Thymopentin ${ }^{124}$ & Peptide & TP5 & Off-line MISPE \\
\hline $\mathrm{HRP}^{125}$ & Protein & HRP & Off-line MISPE \\
\hline Lysozyme $^{126}$ & Protein & Lysozyme & Off-line MISPE \\
\hline $\operatorname{Ricin}^{127}$ & Protein & Ricin A/B Protein chain & Off-line MISPE \\
\hline Hepcidin ${ }^{27}$ & Protein & CGGGHRSKC & Off-line MISPE \\
\hline Human serum transferrin ${ }^{128}$ & Protein & CGLVPVLAENYNK & Off-line MISPE \\
\hline $\mathrm{BiP}^{129}$ & Protein & $\mathrm{BiP}$ & Off-line MISPE \\
\hline Melittin ${ }^{130,131}$ & Peptide & GIGAVLKVLTTGLPALISWIKRKQQ & Off-line MISPE \\
\hline ProGRP 120 & Peptide & Z-NLLGLIEA[Nle] & On-line MISPE \\
\hline Angiotensin $\|^{117}$ & Peptide & Angiotensin II & On-line MISPE \\
\hline $\mathrm{B} 2 \mathrm{M}^{24}$ & Protein & MIQRTPKIQ & On-line MISPE \\
\hline Oxytocin ${ }^{11}$ & Peptide & YPLG & On-line MISPE \\
\hline
\end{tabular}




\subsubsection{Magnetic MIPs (mMIPs)}

Magnetic SPE was developed to improve upon the off-line MISPE protocol by circumventing laborious and time-consuming steps in the process. Many mMIPs have been developed over the last decade, with whole-protein MIPs dominating the format at present (Table 2). BSA, HSA, haemoglobin, and lysozyme are common targets for imprinting due to their low cost and availability. Magnetic nanoparticles imprinted with BSA were successful in the capture of BSA from calf serum ${ }^{132}$. Similarly, surface modified BSA imprinted MIPs demonstrated good selectivity and affinity for BSA ${ }^{133}$. Magnetic lysozyme core-shell MIPs have been developed for selective capture in real samples (i.e. egg white). The surface imprinted MIPs appear to be the most popular format and have excellent results concerning selectivity.

Peptide imprinted mMIPs have been developed for the biomarkers angiotensin I and $\|^{134}$. The mMIPs were core-shell materials with excellent selectivity and affinity in biological samples. The mMIPs had recoveries $>80 \%$ in human serum and detection limits below the reference levels.

Extraction of proteins and peptides by MMIPs is an exciting alternative to standard micro-and nanoporous particle materials. 
Table 2: MIPs for the extraction of proteins and peptides by magnetic SPE

\begin{tabular}{|c|c|c|c|}
\hline Target & Biomolecule & Template & SPE format \\
\hline Angiotensin $\mathrm{I}^{134}$ & Peptide & DRYIHPFHL & magnetic SPE \\
\hline Angiotensin II ${ }^{134}$ & Peptide & DRVYIHPF & magnetic SPE \\
\hline Bovine Hemoglobin ${ }^{135}$ & Protein & Bovine Hemoglobin & magnetic SPE \\
\hline Bovine Hemoglobin ${ }^{136}$ & Protein & Bovine Hemoglobin & magnetic SPE \\
\hline $\mathrm{BSA}^{132}$ & Protein & BSA & magnetic SPE \\
\hline $\mathrm{BSA}^{133}$ & Protein & BSA & magnetic SPE \\
\hline Hemoglobin ${ }^{137}$ & Protein & Haemoglobin & magnetic SPE \\
\hline $\mathrm{HSA}^{138}$ & Protein & HSA & magnetic SPE \\
\hline Lysozyme $^{139}$ & Protein & Lysozyme & magnetic SPE \\
\hline Lysozyme $^{140}$ & Protein & Lysozyme & magnetic SPE \\
\hline Lysozyme $^{138}$ & Protein & Lysozyme & magnetic SPE \\
\hline Lysozyme $^{141}$ & Protein & Lysozyme & magnetic SPE \\
\hline Rnase $A^{142}$ & Protein & Rnase A & magnetic SPE \\
\hline Hemoglobin ${ }^{143}$ & Protein & Haemoglobin & magnetic SPE \\
\hline
\end{tabular}

\subsubsection{MIP Sensors}

MIP sensors represent a highly developed set of bio-recognition materials with successful capture and analysis of a range of protein and peptide targets (Table 3). The primary format is electrochemical sensors. While MISPE materials require some selectivity to function as the MIPs are coupled directly to the sensor. Sensors combine formats and can incorporate micro-and nanoporous particle materials and magnetic particles with the sensing platform for excellent sensitivity and can by-pass more challenging steps in 
MISPE (e.g. elution). Typically, biosensors imprint whole proteins as this allows for greater specificity and selectivity.

Electrochemical sensors use imprinted electrodes that generate a measurable current upon binding of the target molecule. An electrochemical sensor targeting the cancer biomarkers, epidermal growth factor receptor (EGFR), and vascular endothelial growth factor (VEGF) was developed by whole protein imprinting. The MIP biosensors demonstrated excellent sensitivity with detection limits many orders of magnitude below the human reference level. Similarly, an antibody imprinted sensor was developed with excellent selectivity and affinity towards HIV-1 antibodies in human serum. This immunobiosensor demonstrated recoveries of $>80 \%$ in human serum and excellent detection limits.

Table 3: MIP protein and peptide biosensors

\begin{tabular}{|c|c|c|c|}
\hline Target & Biomolecule & Template & Sensor \\
\hline Collagen ${ }^{144}$ & Protein & CTX-I peptide & Electrochemical \\
\hline Cyt $C^{145}$ & Protein & AYLKKATNE & Electrochemical \\
\hline \multirow[t]{2}{*}{ Cyt P450 146} & Protein & oxidase domain (BMO)/ & Electrochemical \\
\hline & & reductase domain (BMR) & \\
\hline $\mathrm{EGFR}^{147}$ & Protein & EGFR & Electrochemical \\
\hline VEGF $F^{147}$ & Protein & VEGF & Electrochemical \\
\hline Ferritin 148 & Protein & Ferritin & Electrochemical \\
\hline $\mathrm{HSA}^{148}$ & Protein & HSA & Electrochemical \\
\hline REG1B ${ }^{149}$ & Protein & REG1B Peptides 2,4,6 & Electrochemical \\
\hline $\mathrm{TnT}^{150}$ & Protein & Troponin $\mathrm{T}(\mathrm{TnT})$ & Electrochemical \\
\hline HIV-1 antibody ${ }^{26}$ & Protein & Human IgG FC & Electrochemical \\
\hline $\mathrm{HRP}^{22}$ & Protein & HRP & Electrochemical \\
\hline
\end{tabular}




\subsection{Model Proteins}

The model proteins used in the present work can be seen in Table 4. As discussed above, MIPs were synthesised to target signature peptides of the two low-abundant biomarkers, ProGRP and NSE as model proteins for the targeted analyses. These proteins are routine biomarkers with extensive characterisation and validated immunoassays available. LC-MS/MS methods have been developed in the last few years, particularly immunoaffinity MS. In addition to the two biomarkers, BSA was used to generate model nonspecific tryptic peptides to evaluate MIP selectivity.

Table 4: Model proteins used in Paper I-III

\begin{tabular}{ccccc}
\hline Protein & Species & Molecular weight $(\mathrm{kDa})^{\mathrm{a}}$ & $\mathrm{AA}^{\mathrm{a}}$ & Paper \\
\hline ProGRP (isoform 1) & Human & 16.21 & 148 & I, III \\
NSE ( chain) & Human & 42.71 & 391 & II, III \\
BSA & Bovine & 69.29 & 607 & I, II, III \\
& & & & \\
\hline
\end{tabular}

Molecular weight and AA from www.uniprot.org

\subsubsection{Progastrin-releasing peptide (ProGRP)}

ProGRP is an ideal model protein as a fully-validated LC-MS method has been developed for its tryptic peptides (e.g. NLLLGIEAK). ${ }^{151}$ ProGRP is known to be clinically sensitive (i.e. the majority of patients with high levels of ProGRP are in a diseased state) and selective (i.e. the majority of patients with a low level of ProGRP are not in the diseased state). ${ }^{152}$ The signature peptide of ProGRP, NLLGLIEAK, is a very reproducibly produced tryptic peptide and has high MS sensitivity. ProGRP has three distinct isoforms (isoform 1, 2, and 3) with increases in the levels of all three being linked to carcinogenesis (i.e. the development of cancer). ProGRP quantification in MS is ideal as 2 of the isoforms are readily distinguishable. The present work did not focus on isoform distinction, instead, using isoform 1 (the most common in human serum) and the signature peptide NLLGLIEAK, common for all isoforms, for quantification in bottom-up proteomics. Isoform 1 contains three signature peptides, NLLGLIEAK, ALGNQQPSWDSEDSSNFK, and LSAPGSQR (Fig. 5) 


\section{VPLPAGGGTVLTKMYPRGNHWAVGHLMGKKSTGESSSVSERGSLKQQLR EYIRWEEAARNLLGLIEAKENRNHQPPQPKALGNQQP SWDSEDSSNFKD VGSKGKVGRLSAPGSQREGRNPQLNQQ}

Figure 5: Sequence of ProGRP isoform 1: The signature peptides monitored in the present study, NLLGLIEAK (orange) and ALGNQQPSWDSEDSSNFK (blue) are common to all isoforms. LSAPGSQR (red) is specific for isoform 1.

ProGRP levels in excess of $70 \mathrm{pM}$ in serum are considered to be in the disease range ${ }^{153}$. The ProGRP reference level is approximately $7 \mathrm{pM}^{152}$. As ProGRP is a low-abundant protein, the MISPE must be able to capture NLLGIEAK selectively and provide a clean sample for MS/MS analysis. The present work used isoform 1 to quantify ProGRP in human serum. In Paper I all three signature peptides were used: NLLGLIEAK to quantify ProGRP while ALGNQQPSWDSEDSSNFK and LSAPGSQR were used to evaluate MIP selectivity. In Paper III, isoform 1 and NLLGIEAK was used to quantify ProGRP in human serum.

\subsubsection{Neuron-specific Enolase (NSE)}

NSE is the second major SCLC biomarker used in the present study. NSE is a less specific biomarker than ProGRP as it is present in elevated levels in a range of cancers (e.g. neuroblastoma, melanoma, and breast cancer $^{109}$. It is found in elevated levels in SCLC patients and so is used alongside ProGRP in the diagnosis of SCLC. The use of both NSE and ProGRP improves the specificity of the SCLC diagnosis. Therefore, typically elevated levels of both biomarkers are needed for accurate determination of SCLC Enolases are dimeric proteins found in a range of human tissues. There are three unique subunits $(\alpha, \beta$, and $\gamma)$ that can form five unique isoenzymes $(\alpha \alpha, \alpha \beta, \alpha \gamma, \beta \beta \text {, and } \gamma \gamma)^{154}$. The $\gamma \gamma$ heterodimer is NSE (also known as enolase 2) and is expressed at high levels in neural tissue. NSE also presented an opportunity to build on the work done of ProGRP MIPs and develop duplex methods for analysis of SCLC biomarkers.

NSE is an attractive model system for MISPE materials. MIPs targeting NSE have been developed for use in electrochemical sensors ${ }^{155}$, but not for off-line or on-line MISPE in combination with MS/MS analysis. NSE has a long sequence (Fig. 6) with several potential signature peptides but MS/MS methodology has been developed specifically targeting the signature peptide, ELPLYR ${ }^{109}$. Therefore, MIPs targeting ELPLYR were designed and synthesised for the present study. ELPLYR is the primary signature peptide used in bottom-up analysis due to its specificity combined with strong ionisation in electrospray ionisation ${ }^{109}$. 
MS IEKIWARE I LDSRGNPTVEVDLYTAKGLFRAAVPSGASTGIYEALEL RDGDKQRYLGKGVLKAVDHINSTIAPALISSGLSVVEQEKLDNLMLELD GTENKSKFGANAILGVSLAVCKAGAAERELPLYRHIAQLAGNSDLILPV PAFNVINGGSHAGNKLAMQEFMILPVGAESFRDAMRLGAEVYHTLKGVI KDKYGKDATNVGDEGGFAPNI LENSEALELVKEAIDKAGYTEKIVIGMD VAASEFYRDGKYDLDFKSPTDPSRYITGDQLGALYQDFVRDYPVVSIED PFDQDDWAAWSKFTANVGIQIVGDDLTVTNPKRIERAVEEKACNCLLLK VNQIGSVTEAIQACKLAQENGWGVMVSHRSGETEDTFIADLVVGLCTGQ IKTGAPCRSERLAKYNQLMRIEEELGDEARFAGHNFRNPSVL

Figure 6: Sequence of NSE: The signature peptide ELPLYR (orange) monitored in the present study,

As with ProGRP, a MISPE method must be able to selectively capture ELPLYR as NSE is a low-abundant biomarker and clean extracts are needed for quantification below the reference level of approximately 250 pM in human serum ${ }^{156}$.

\subsubsection{MS/MS Analysis of the Model Proteins}

The analysis of the biomarkers in the present study was exclusively done through bottom-up proteomics. The selected instrument for all the analyses was a triple quadrupole MS. As described in section 1.3.3, triple quadrupole MS allows for excellent selectivity in the analysis of tryptic peptides. By controlling the $m / z$ values that can pass through $\mathrm{Q} 1$ and Q3, and the collision energy, the resultant mass spectra, and chromatograms are non-complex. As such, the quantification of the signature peptides is fast and easy. The parameters used for each biomarker and their signature peptides can be found in table 5. 
Table 5: The monitored signature peptides: sequence, precursor mass $(m / z)$, product mass $(m / z)$, and collision energy.

\begin{tabular}{|c|c|c|c|c|c|}
\hline Protein & $\begin{array}{l}\text { Peptide } \\
\text { sequence }\end{array}$ & $\begin{array}{l}\text { Ionisation } \\
\text { Mode }\end{array}$ & $\begin{array}{l}\text { Precursor mass } \\
(\mathrm{m} / \mathrm{z})\end{array}$ & $\begin{array}{l}\text { Product mass } \\
\qquad(\mathrm{m} / \mathrm{z})\end{array}$ & $\begin{array}{c}\text { Collision energy } \\
\text { (V) }\end{array}$ \\
\hline \multirow[t]{10}{*}{ ProGRP } & NLLGLIEAK & Positive & 485.800 & 630.300 & 15 \\
\hline & & & & 743.400 & 16 \\
\hline & NLLGLIEAK IS & Positive & 489.900 & 638.300 & 15 \\
\hline & & & & 751.400 & 16 \\
\hline & LSAPGSQR & Positive & 408.200 & 272.600 & 17 \\
\hline & & & & 544.400 & 14 \\
\hline & ALGNQQPSW- & Positive & 1005.450 & 595.300 & 35 \\
\hline & 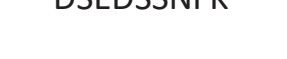 & & & 913.300 & 35 \\
\hline & & & & 1028.300 & 35 \\
\hline & & & & 1398.500 & 35 \\
\hline \multirow[t]{4}{*}{ NSE } & ELPLYR & Positive & 395.700 & 274.700 & 14 \\
\hline & & & & 548.300 & 14 \\
\hline & ELPLYR IS & Positive & 401.000 & 297.700 & 14 \\
\hline & & & & 558.300 & 14 \\
\hline \multirow[t]{7}{*}{ BSA } & LVTDLTK & Positive & 395.240 & 577.360 & 30 \\
\hline & AEFVEVTK & Positive & 461.750 & 722.430 & 30 \\
\hline & HPEYAVSLLR & Positive & 642.360 & 920.610 & 30 \\
\hline & HLVDEPQNLIK & Positive & 653.360 & 1055.590 & 30 \\
\hline & YICDNQDTISSK & Positive & 722.820 & 1168.450 & 30 \\
\hline & LGEYGFQNALIVR & Positive & 740.400 & 1017.530 & 30 \\
\hline & DAFLGSFLYEYSR & Positive & 784.380 & 1121.560 & 30 \\
\hline
\end{tabular}




\section{Aim of the Study}

The present study aimed to develop and evaluate novel MIPs in multiple formats for use as extraction materials coupled to LC-MS/MS quantification of low abundant biomarkers from human serum. The key areas of interest were the synthesis of the MIPs, the development of a selective SPE protocol with downstream compatibility in complex matrices, quantification of SCLC biomarkers, and the performance of the MIPs in the extraction in human serum. The latter focused on the key parameters of extraction recovery, the limit of detection (LOD), the limit of quantification (LOQ), linearity, and method accuracy. The following MIP-SPE formats were evaluated:

- Magnetic MIPs in the quantification of ProGRP (Paper I)

- On-line MIPs in the extraction of ProGRP and NSE (Paper II, and Paper III)

- Combination of MIPs targeting both ProGRP and NSE in an on-line format (Paper III)

The following research questions were answered in the present study:

- What is the ideal synthetic strategy for microporous MIPs for use as SPE materials?

- What are the ideal conditions for selective extraction of signature peptides for SCLC biomarkers? (Paper I, Paper II, Paper III)

- How selective were the MIPs? (Paper I, Paper II, Paper III)

- Can MIPs targeting signature peptides, NLLGLIEAK and ELPLYR, be used in the quantification of ProGRP and NSE in human serum? (Paper I, Paper II, Paper III)

- Can MIPs be combined in a single extraction for duplex analysis of ProGRP and NSE? (Paper III) 


\section{Results and Discussion}

\subsection{MIP Preparation}

MIPs were synthesised for the specific capture of both NLLGLIEAK and ELPLYR, the signature peptides of ProGRP and NSE, respectively. The MIPs in Paper / were mMIPs designed for use in off-line magnetic SPE. MIPs targeting ELPLYR were used in on-line SPE in Paper II and Paper III. Finally, a MIP targeting NLLGLIEAK for on-line SPE was used in Paper III. The synthesis of these MIPs will be discussed, including the rationale of template and FM selection, synthetic scheme, and for Paper II and Paper III, column packing.

\subsubsection{Template Selection}

The careful choice of template is essential in MIP synthesis. Considerations must be given to structural similarity to the target molecule, cost and availability, solubility in synthetic solvents, conformation and conformational rigidity, stability, and functional groups available for templating (i.e. have functional groups present that can be exploited for selective and specific interactions with the target molecule). MIPs as SPE materials present an added challenge; namely, template bleeding during the elution phase of the extraction ${ }^{157}$. Template bleeding occurs when a template that remains in the 3D network during synthesis is not removed sufficiently during the template removal step. During the elution step of the SPE protocol, this template can bleed into the eluent. This leads to an increase in the signal of the target molecule, and potentially a false positive. To circumvent this, MIPs for use in MS analysis can be produced using a modified template structure. This will prevent any increase in false positives as the MS can easily distinguish between the modified template and native target molecule. However, changes in the imprinting structure may affect selectivity and specificity due to the differences between the modified and native peptides.

In Paper I and Paper III a modified peptide (i.e. Z-NLLGLIEA[Nle]) was used in the synthesis of the magnetic MIP and MIP polymer respectively. Figure 7A illustrates the native NLLGLIEAK structure and the modified template used in Paper I and Paper III. A carboxyphenyl group has been added to the $\mathrm{N}$-terminus for added solubility in the organic solvent. The Z-NLLGLIEA[Nle] C-terminus lysine was replaced with norleucine. This was done to enhance binding strength and improve selectivity (i.e. by preventing all tryptic peptides having some partial selectivity). All tryptic peptides will generate a partially positively charged C-terminus due to the mechanism of trypsin, which cleaves proteins at a lysine or arginine group. The lysine present on the native peptide could repel functional groups with similar positive charges. The norleucine, however, will react with positively charged functional monomers. In Paper II and Paper III, the rationale in template design for ELPLYR MIPs was based upon previously reported NLLGLIEAK MIPs ${ }^{158}$. The modified 
template Z-ELPLY[Nle] (Fig. 7B) similarly has a carboxyphenyl at the N-terminus and a norleucine at the Cterminus. The effects of altering the template on binding strength and selectivity towards the native NLLGLIEAK or ELPLYR is unknown. The partially positively charged lysine and arginine present in the NLLGLIEAK and ELPLYR during extraction and may cause some partial repulsion with the MIP binding sites.

A)

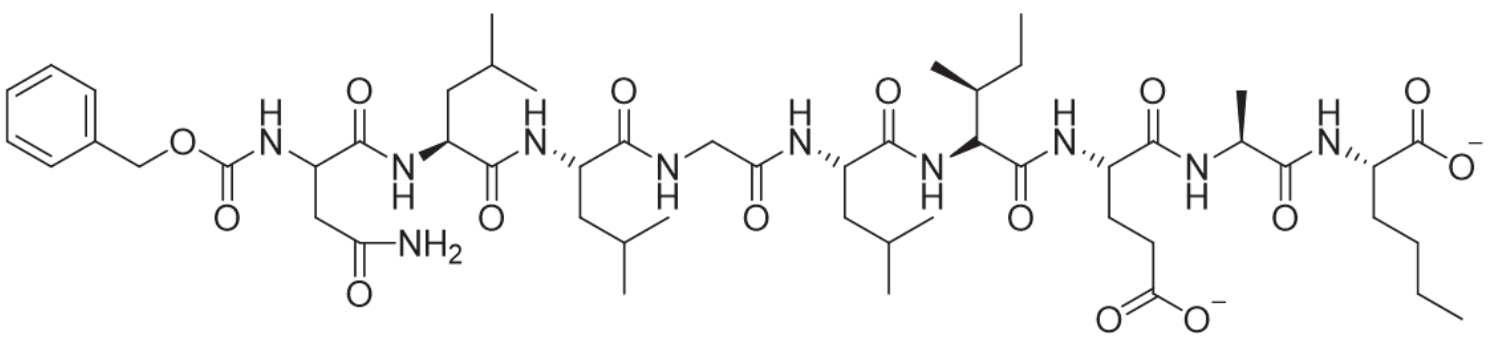

B)<smiles>CCCCC(NC(=O)[C@H](Cc1ccc(O)cc1)NC(=O)[C@H](CC(C)C)NC(=O)[C@@H]1CCCN1C(=O)[C@H](CC(C)C)NC(=O)C(CCC(=O)[O-])NC(=O)OCc1ccccc1)C(=O)[O-]</smiles>

Figure 7: A) The modified template used for the MIPs to capture NLLGLIEAK (Paper I and Paper III). A carboxyphenyl group has been added to the $\mathrm{N}$-terminus and the $\mathrm{C}$-terminus $\mathrm{K}$ has been replaced by norleucine. B) The modified template was used for the MIPs to capture ELPLYR (Paper II and Paper III). A carboxyphenyl group has been added to the $\mathrm{N}$-terminus and the $\mathrm{C}$-terminus $R$ has been replaced by norleucine.

\subsubsection{Functional Monomer Selection}

MIPs were designed and synthesised for the selective capture of either NLLGLIEAK or ELPLYR. The specific retention mechanism selected for both NLLGLIEAK and ELPLYR MIPs was interactions via delocalised negative charges of the carboxylic acid functional groups found in the glutamic acid. Therefore, any selected FMs must be able to form delocalised positive charges that are accessible for MIP-peptide interactions. The FMs used in the present study were $\mathrm{N}$-(2-aminoethyl) methacrylamide hydrochloride (EAMA.HCl), (trifluoromethyl)-phenyl- $\mathrm{N}^{\prime}$-4-vinylphenylurea (TFU), with divinylbenzene-80 (DVB-80) as CL in all MIPs (Fig. 8). 
<smiles>C=Cc1ccccc1C=C</smiles>

DVB-80<smiles>C=C(C)C(=O)NCCN</smiles>

EAMA. $\mathrm{HCl}$<smiles>C=Cc1ccc(NC(=O)Nc2cc(C(F)(F)F)cc(C(F)(F)F)c2)cc1</smiles>

TFU

Figure 8: the FMs and cross-linker used to synthesise the MIPs used in Papers I, II, and III.

\subsubsection{Magnetic MIP Synthesis}

The polymers synthesised by precipitation polymerization were prepared at the Department of Pure and Applied Chemistry, University of Strathclyde, United Kingdom.

Four magnetic MIPs were synthesised for use in magnetic SPE in Paper I (Table 6). Concurrent to MIP synthesis, non-imprinted polymers (NIPs) were synthesised as control polymers. NIP synthesis is identical to MIP synthesis but does not contain the template molecule.

Magnetic core-shell MIP microspheres (mMIP A and MMIP B) were synthesised by a two-step precipitation polymerization (PP). Porous poly(methacrylic acid-co-DVB-80) microspheres were initially prepared before the magnetic component (i.e. $\mathrm{Fe}_{3} \mathrm{O}_{4}$ ) was incorporated into the microspheres. This was done by filling the pores with $\mathrm{Fe}_{3} \mathrm{O}_{4}$. The magnetised poly(MAA-co-DVB-80) microspheres were then used as a core for which a second PP, in the presence of the template, yielded magnetic core-shell MIP microspheres. The FMs for mMIP A were EAMA.HCl and TFU, while mMIP B used only EAMA.HCl.

The polymers, mMIP C and mMIP D, and their corresponding NIPs, were prepared by magnetization of imprinted and non-imprinted porous polymer microspheres which had been produced via a PP protocol. Therefore, the first step was the synthesis of porous MIP microspheres (and their corresponding NIPS) with Z-NLLGLIEA[Nle] as the template, which was followed by the magnetization procedure. The MIPs and NIPs were synthesised according to a PP method described elsewhere ${ }^{120}$. Particle size and dispersity were determined by scanning electron microscopy (SEM).

The two formats (post magnetization and magnetic core-shell) were selected to determine the optimal mMIP format. As described earlier, the post-magnetisation is often preferred due to the pre-determined effectiveness of the MIPs. However, the core-shell MIPs are larger and monodisperse with all core-shell materials having sizes between 4 and $5 \mu \mathrm{m}$. Monodispersity can reduce variability as there is much more even distribution of particles during MIP aliquoting before analysis. The greater size provides more surface 
area and therefore, potentially a greater binding capacity. However, as the post-magnetised MIPs are smaller and more polydisperse (i.e. 1-5 $\mu \mathrm{m}$ ), they may have a lower capacity compared to pre-imprinted variants. A key aim of the present work is to determine ideal MIPs synthesis strategies.

Table 6: mMIP and mNIP structural information used in Paper I

\begin{tabular}{|c|c|c|c|c|}
\hline Polymer & Components & Template & Dispersity & Bead Size $(\mu \mathrm{m})$ \\
\hline $\mathrm{mMIPA}$ & $\begin{array}{l}\text { DVB-80, EAMA.HCl, } \\
\text { PMP }\end{array}$ & Z-NLLGLIEA[Nle] & Polydisperse & $4-5$ \\
\hline mNIP A & $\begin{array}{l}\text { DVB-80, EAMA.HCl, } \\
\text { PMP }\end{array}$ & - & Polydisperse & $4-5$ \\
\hline mMIP B & $\begin{array}{l}\text { DVB-80, TBA.OH, } \\
\text { TFU, EAMA.HCI, PMP }\end{array}$ & Z-NLLGLIEA[Nle] & Polydisperse & $4-5$ \\
\hline mNIP B & $\begin{array}{l}\text { DVB-80, TBA.OH, } \\
\text { TFU, EAMA.HCI, PMP }\end{array}$ & - & Polydisperse & $4-5$ \\
\hline mMIP C & $\begin{array}{l}\text { DVB-80, EAMA. } \mathrm{HCl} \text {, } \\
\text { PMP }\end{array}$ & Z-NLLGLIEA[Nle] & Polydisperse & $4-5$ \\
\hline mNIP C & $\begin{array}{l}\text { DVB-80, EAMA.HCl, } \\
\text { PMP }\end{array}$ & - & Polydisperse & $1-5$ \\
\hline mMIP D & $\begin{array}{l}\text { DVB-80, TBA.OH, } \\
\text { TFU, EAMA.HCl, PMP }\end{array}$ & Z-NLLGLIEA[Nle] & Polydisperse & $\approx 1$ \\
\hline mNIP D & $\begin{array}{l}\text { DVB-80, TBA.OH, } \\
\text { TFU, EAMA.HCI, PMP }\end{array}$ & - & Polydisperse & $\approx 1$ \\
\hline
\end{tabular}

DVB-80 = divinyl benzene; EAMA. $\mathrm{HCl}=\mathrm{N}$-(2-Aminoethyl)methacrylamide; $\mathrm{PMP}=$ pentamethylpentane; $\mathrm{TBA} . \mathrm{OH}=$ Tetrabutylammonium hydroxide; TFU = trifluoromethyl)-phenyl- $\mathrm{N}^{\prime}-4$-vinylphenylurea

\subsubsection{Microparticle MIP Synthesis}

Two Z-ELPLY[Nle] imprinted polymers (ELPLYR-MIP 1 and ELPLYR-MIP 2) and their corresponding NIPs (NIP ELPLYR-1 and ELPLYR-NIP 2) were prepared in a microsphere format using the reagents outlined in Table 7 by PP. Two different template to functional monomer mole ratios were used (1:4.7 for ELPLYR-MIP 1 and 1:9.3 for ELPLYR-MIP 2), to discern any influence on the molecular recognition capabilities of the products. Interestingly, the product yield was considerably higher than the yield of similar photopolymerised polymers previously reported ${ }^{159}$. The size and dispersity were determined by SEM. 
The purpose of the microporous MIPs was for packing into columns for on-line SPE. Therefore, the particles must have good size and low polydispersity. Smaller size particles ensure greater surface area (i.e. greater capacity) and allow for packing into smaller columns that are compatible with microflow LC systems. The ELLPLYR imprinted MIPs (and their corresponding NIPs) had sizes ranging from 2.2 and 2.8 $\mu \mathrm{m}$. However, the particles were polydisperse. This may impact packing bed homogeneity. TFU was selected as the primary monomer as it has been shown to bind strongly to negatively charged groups on AAs such as the glutamate found in ELPLYR.

The synthesis of the Z-NLLGLIEA[Nle] imprinted MIP i.e. NLLGLIEAK-MIP (and its corresponding NLLGLIEAK-NIP) used in Paper III has been previously described ${ }^{120}$.

Table 7: Polymer MIPs and NIPs structural information used in Paper II and Paper III

\begin{tabular}{|c|c|c|c|c|c|}
\hline Polymer & Components & Template & Dispersity & $\begin{array}{c}\text { Bead Size } \\
(\mu \mathrm{m})\end{array}$ & Paper \\
\hline ELPLYR-MIP 1 & $\begin{array}{l}\text { DVB-80, TFU, } \\
\text { PMP, TBA.OH }\end{array}$ & Z-ELPLY[Nle] & Polydisperse & 2.8 & II, III \\
\hline ELPLYR-NIP 1 & $\begin{array}{c}\text { DVB-80, TFU, } \\
\text { TBA.OH }\end{array}$ & - & Polydisperse & 2.4 & II, III \\
\hline ELPLYR-MIP 2 & $\begin{array}{l}\text { DVB-80, TFU, } \\
\text { PMP, TBA.OH }\end{array}$ & Z-ELPLY[Nle] & Polydisperse & 2.8 & II \\
\hline ELPLYR-NIP 2 & $\begin{array}{c}\text { DVB-80, TFU, } \\
\text { TBA.OH }\end{array}$ & - & Polydisperse & 2.2 & II \\
\hline NLLGLIEAK-MIP & $\begin{array}{c}\text { DVB-80, } \\
\text { EAMA.HCl, PMP }\end{array}$ & Z-NLLGLIEA[NIe] & polydisperse & $\mathrm{n} / \mathrm{a}$ & III \\
\hline NLLGLIEAK-NIP & $\begin{array}{c}\text { DVB-80, } \\
\text { EAMA.HCl, PMP }\end{array}$ & & polydisperse & $\mathrm{n} / \mathrm{a}$ & III \\
\hline
\end{tabular}

DVB-80 = divinyl benzene; EAMA. $\mathrm{HCl}$ = N-(2-Aminoethyl)methacrylamide; PMP = pentamethylpentane; TBA.OH = Tetrabutylammonium hydroxide; TFU = trifluoromethyl)-phenyl- $\mathrm{N}^{\prime}$-4-vinylphenylurea 


\subsubsection{Column Packing}

For on-line analysis in Paper II and Paper III, the polymers required packing into trap columns for automated SPE. Polydisperse particles (such as the MIPs in the present study) can often have irregular packing due to the irregular shapes and sizes of the particles forming cavities and disruptions to the packing bed. Such disruptions may lead to poor extraction by the MIPs. If these disruptions are present, target analytes may pass through these cavities as it is likely to be a path of least resistance. This can lead to reductions in retention factor, $\mathrm{k}$. On-line MIPs use $\mathrm{k}$ to determine the selectivity of the material and hence it is essential to evaluate the packing quality before performing any SPE. This can be achieved by optical microscopy to visually assess the packing bed, peak symmetry $(A$,$) analysis, and measurement of$ column backpressure. Peak symmetry analysis is performed by passing a tracer molecule (i.e. a molecule that will not interact with polymer) through the packed column and using a UV detector to generate a peak. If the peak is symmetrical (i.e. $A, \leq 1.5$ ), the packing bed likely has little to no packing irregularities. ELPLYR-MIP 1, ELPLYR-NIP 1 ELPLYR-MIP 2, and ELPLYR-NIP 2 all head good peak symmetry (i.e. A, $\leq 1.5$ ). Furthermore, the backpressures were between 12 and $24 \mathrm{PSI}$, suggesting there are no unexpected channels in the packing bed.

The NLLLGLIEAK-MIP was previously optimised as an on-line SPE column ${ }^{120}$ and so peak symmetry was not assessed in the present study. 


\subsection{MISPE Method Development}

Novel MIPs have unpredictable properties and careful consideration must be given to the extraction conditions. A common theme in Papers I, II, and III is the development of specific MISPE methods that have both good selectivity and affinity towards the target peptide. The following section will highlight and discuss the process of developing the MISPE methods; both on- and off-line.

\subsubsection{Magnetic SPE of NLLGLIEAK (Paper $)$}

The extraction of NLLGLIEAK by mMIPs was the focus of Paper I. The magnetic-SPE of NLLGLIEAK was an off-line extraction with three major steps prior to MS/MS analysis: extraction, wash, and elution. The initial strategy involved using NLLGIEAK IS (heavy-isotope labelled) in a simple matrix; namely, $50 \mathrm{mM}$ ammonium bicarbonate buffer $(A B C)$ and only one MIP. Using one MIP allowed for the initial conditions to be determined and later used to assess the optimal MIP. ABC buffer was used as the matrix to ensure the capture, wash, and elution of NLLGLIEAK was not influenced by matrix components. ABC buffer was selected as the matrix for downstream compatibility with serum extractions. $A B C$ buffer has an ideal $\mathrm{pH}$ (7.8) for tryptic digestion. As the binding mechanism of the mMIPs are $\mathrm{pH}$ dependant, it is essential to perform the initial optimisation in a pH similar to the application matrix (e.g. serum). MIPs are synthesised in an organic environment and specific binding sites may be affected by an aqueous environment (e.g. $A B C$ buffer). To determine if the mMIPs maintain functionality in an aqueous matrix, the recoveries of NLLGIEAK ( $5 \mathrm{nM}$ ) in both organic solvent (acetonitrile, MeCN) and an aqueous matrix (50 mM $A B C$ ) were measured and compared. The binding efficiency (i.e. \% bound analyte) was found to be $99.9 \%$ for both the aqueous and organic extractions, respectively. This suggests that there are not major observable effects on the binding sites of the MMIP in an aqueous environment.

\section{Magnetic MIP Extraction Optimization}

Determining the minimum sorbent amount and extraction time is an essential aspect of mMIP extraction optimisation. Shorter extraction times are essential for analytes with limited stability, but also ensure higher throughput. Ensuring the lowest amount of sorbent ensures binding capacity and costeffectiveness are maximised. Three mMIP amounts and extraction times were investigated to maximise the binding efficiency. The optimal extraction time was found to be $5 \mathrm{~min}$ when using $600 \mu \mathrm{g}$ of the sorbent.

\section{Magnetic MIP selection}

Four MIPs were produced (mMIP A, mMIP B, mMIP C, and mMIP D) along with their corresponding nonimprinted counterparts. The best performing MIP was selected based on selectivity (i.e. the binding 
efficiency of the MIP with respect to its corresponding NIP). Selectivity was used as the key parameter as it has been reported that it is a determining factor on whether MIPs will function in complex matrices ${ }^{160}$. BSA was added to the matrix to block non-specific binding sites and ensure the binding was primarily specific. The most selective polymer was MMIP C, a material that used EAMA.HCl as the primary FM (Fig. 9). Among the mMIP/mNIP pairs, only mMIP C had a significantly higher affinity towards NLLGLIEAK than its corresponding NIP. The use of EAMA. $\mathrm{HCl}$ has been previously shown to be highly effective in the capture of peptides. ${ }^{120}$

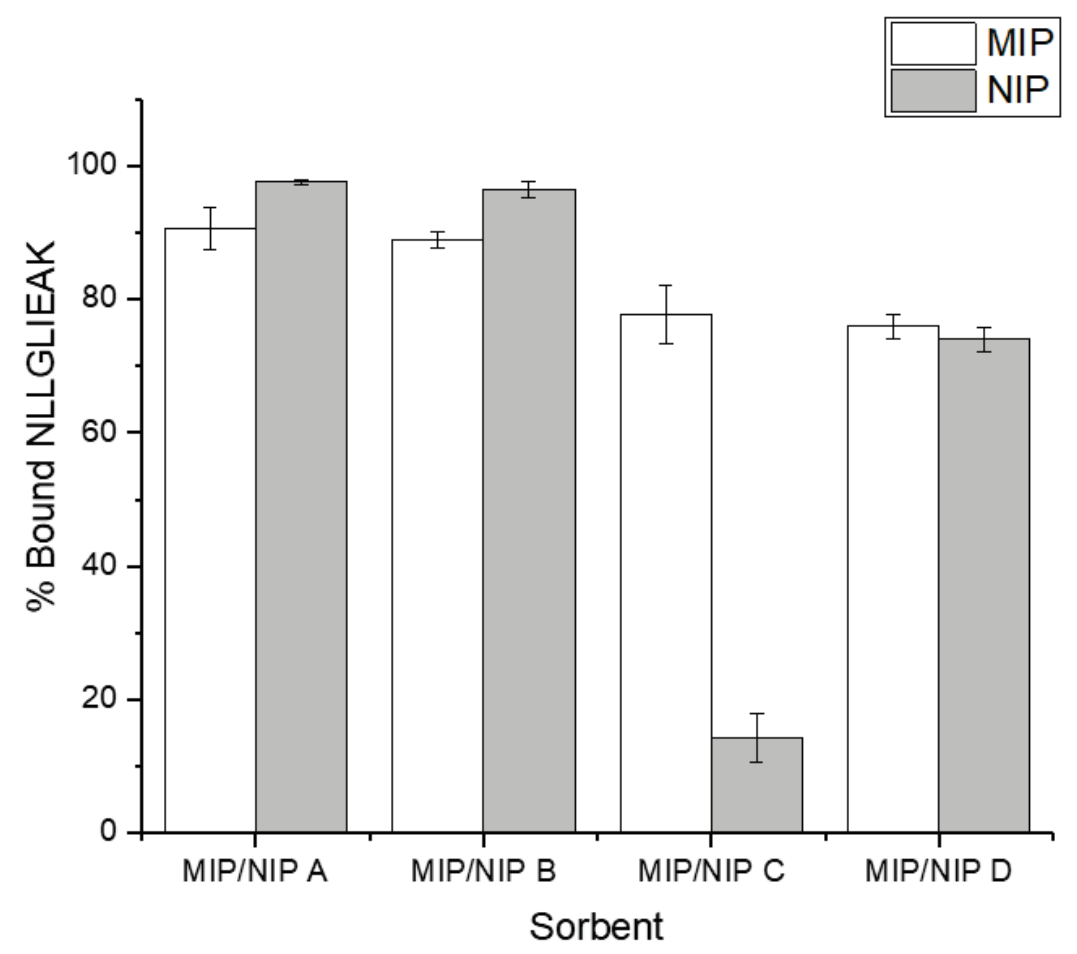

Figure 9: Selectivity of the mMIP/mNIP pairs towards target peptide determined as binding efficiency (\% bound NLLGLIEAK IS \pm standard deviation of NLLGLIEAK IS). Samples consisted of NLLGLIEAK IS (5 nM) in ABC (50 mM) containing $10 \mathrm{nM}$ digested BSA ( $\mathrm{n}=3)$. Reproduced with permission from Fig. 1 Paper I.

\section{Wash and Elution}

Washing is essential in SPE to remove non-specifically bound peptides and other adsorbed matrix components. The removal of these unwanted components from the polymer before elution ensures a cleaner extract for MS/MS analysis. Low-abundant biomarkers often require cleaner extracts as this contributes to lower detection limits. However, a wash that is too strong may lead to loss of the target peptide. As such, compromises between the loss of target peptide and efficient removal of adsorbed 
compounds must be balanced. To find this optimal balance, increase in MeCN was added to $50 \mathrm{mM} \mathrm{ABC}$ $(0,1,2,3,4$ and $5 \% \mathrm{MeCN}, \mathrm{n}=3)$. There was a linear relationship between increases in the \%MeCN and loss of NLLGLIEAK, with $49.7 \pm 0.3 \%$ lost NLLGLIEAK at $5 \%$ MeCN (Fig. 10). Conversely, at $0 \% \mathrm{MeCN}$, the loss of NLLGLIEAK was limited to only $2.2 \pm 1.6 \%$. This suggests that even small increases in the amount of organic solvent can disrupt the binding of NLLGLIEAK. While increases in MeCN are more likely to remove non-specific peptides the loss of NLLGLIEAK is too high at even $1 \% \mathrm{MeCN}$. Therefore, the washing solution was selected to be $100 \%$ aqueous (i.e. $50 \mathrm{mM} \mathrm{ABC}$ ).

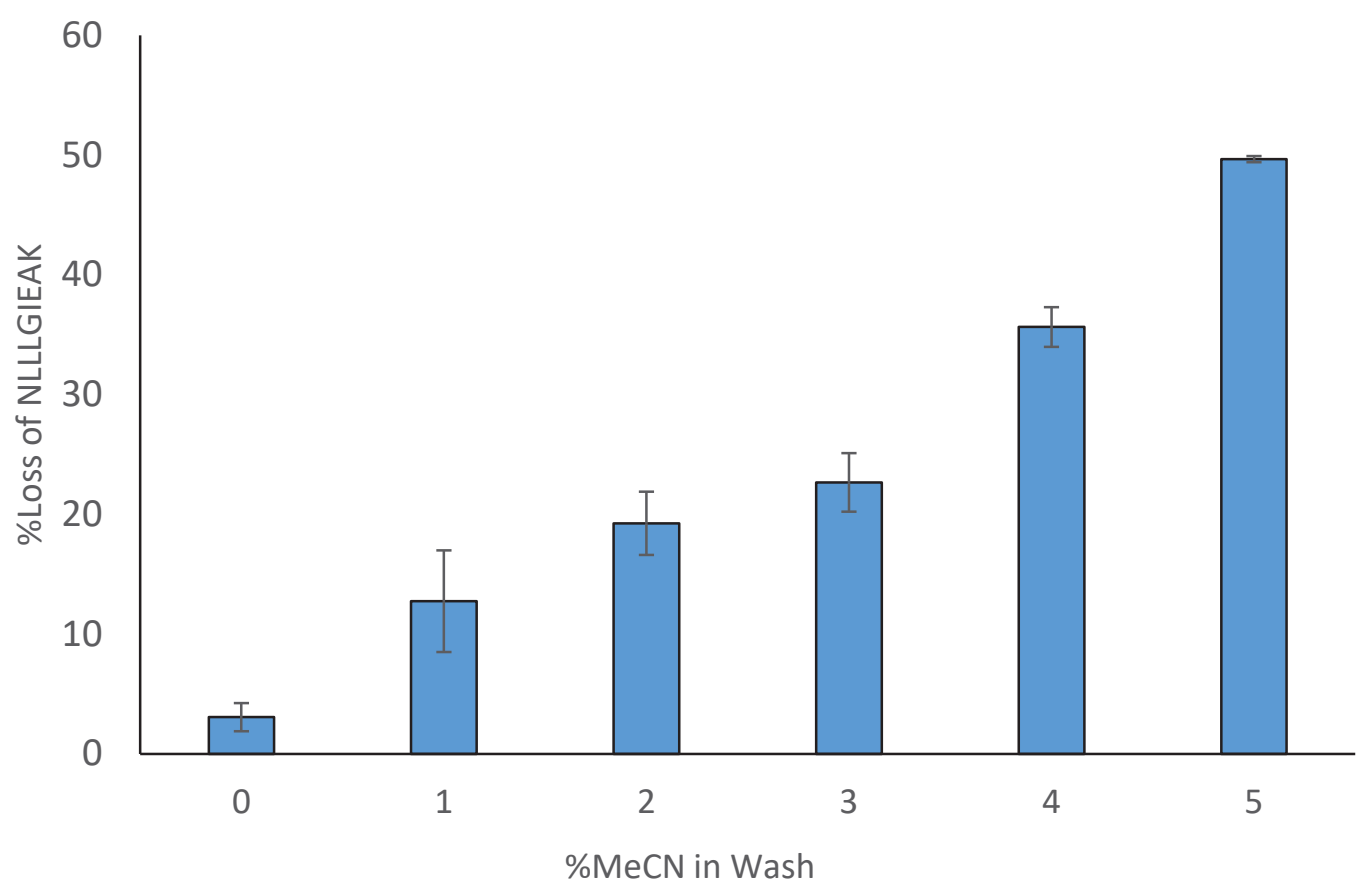

Figure 10: Samples were washed with buffers containing 0, 1, 2, 3, 4, and 5\% MeCN (n=3). Reproduced with permission from Fig. 4 Paper 1.

Perhaps the most challenging aspect of off-line MISPE is the elution step. As outlined in section 1.1, many of the binding sites can be found deep within the 3D matrix of the polymer network. As a consequence, any bound target analyte may get trapped in the network and may be unable to elute from the polymer. mMIP C is not a core-shell polymer, therefore, many of the binding sites would reasonably be expected to be found below the surface. Therefore, the wash solution may still trap some NLLGLIEAK. As the interaction between mMIP C and NLLGLIEAK occurs via a delocalised negative charge on the glutamate of 
NLLGLIEAK and the delocalised positive charge of the EAMA.HCl of the MIP, a drop in pH should disrupt this bond due to protonation of the glutamate group. This can be seen when an elution solution containing 7.5:92.5 MeCN: $\mathrm{H}_{2} \mathrm{O}$ only gave a recovery of $2.5 \pm 4.3 \%$. Interestingly, in previous wash experiments, $5 \%$ MeCN led to a high loss of NLLGLIEAK yet 7.5\% did not elute high amounts of NLLGLIEAK. This may be due to multiple aqueous washes which trap NLLGLIEAK deep in the MIP structure. This is further seen when the addition of $0.1 \%$ FA to the elution solution only gave a recovery of $6.2 \pm 10.7 \%$. A more organic environment would be expected to restore the structure of the MIP and, with an increase of $\mathrm{pH}$, release the bound NLLGLIEAK. This can be observed with the elution solution comprised of 80:15:5 MeCN: $\mathrm{H}_{2} \mathrm{O}: \mathrm{FA}$. Following elution with this solution, the recovery increased to $84.8 \pm 14.1 \%$. The $85 \% \mathrm{MeCN}$ component is likely to play a role in elution as hydrophobic interactions play a role in NLLGLIEAK binding. Therefore, increases in the organic solvent will disrupt any hydrophobic interactions and release NLLGLIEAK into the elution solution.

\subsubsection{On-line SPE of NLLGLIEAK and ELPLYR (Paper // and III)}

On-line MISPE involves the coupling of MIPs to the LC system to act as an automated extraction system. There are two pumps, a loading and microflow pump, and two columns: a MIP trap column and an analytical column (i.e. C18). The sample is loaded and extracted by the MIP in the first stage before the MIP is connected to the analytical column and the bound analytes are forward flushed onto the analytical column. The analytes are separated by reverse phase chromatography before analysis by a triple quadrupole MS (Fig. 11). 

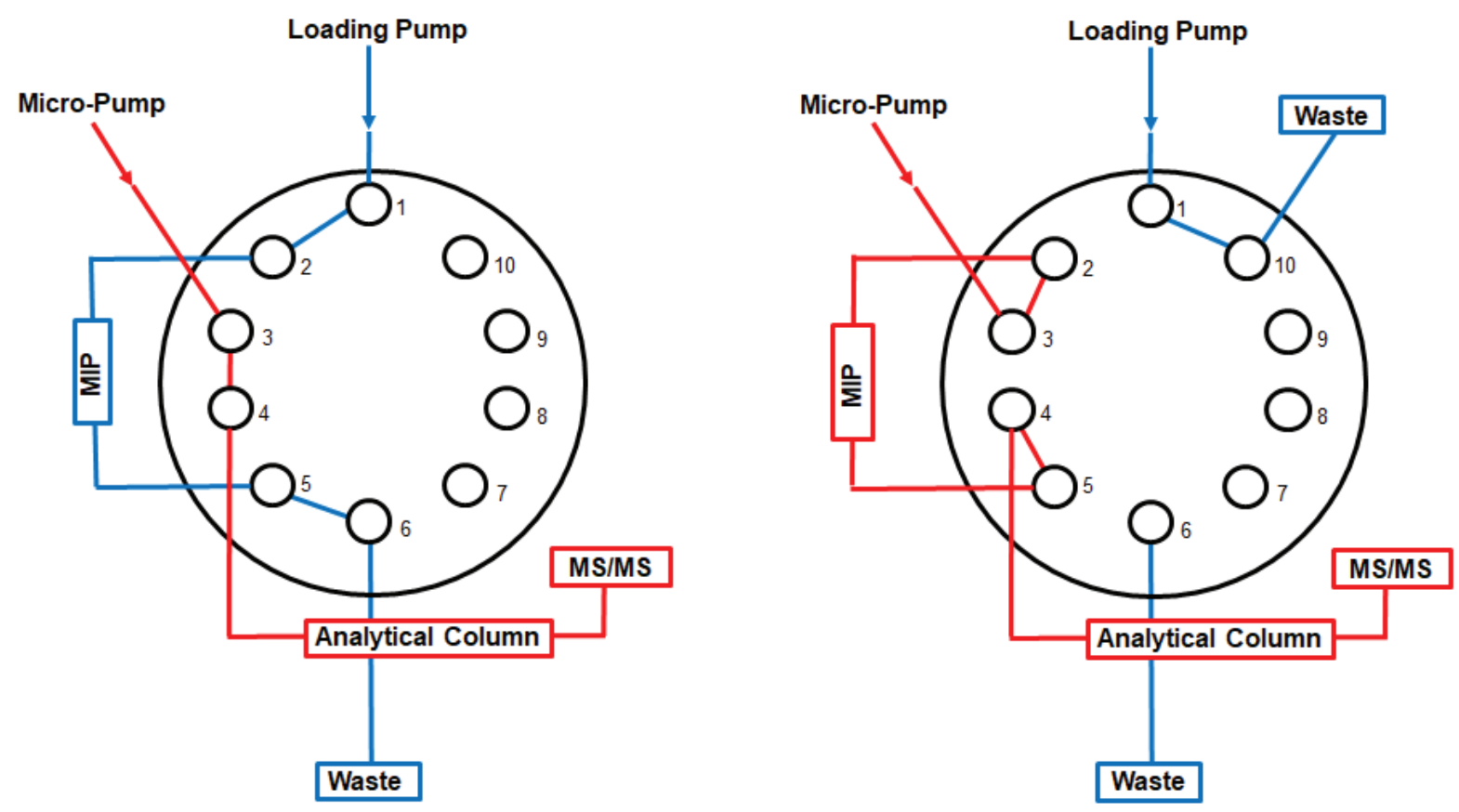

Figure 11: The on-line MISPE system. Firstly, the analyte is injected onto the MIP (left). Following sample loading, the system switches and the analyte is gradient eluted onto a C18 analytical column before determination by a triple quadrupole MS.

\section{Loading Mobile Phase}

There are several major differences in on-line and off-line setups that determine how the MISPE method is optimised. In Paper I, mMIPs were added to a matrix for extraction. However, in on-line SPE, it is reversed with the matrix containing the sample added to the MIPs while a loading mobile phase passes through. As with the mMIPs, the matrix selected for extraction optimisation was $50 \mathrm{mM} \mathrm{ABC}$ buffer. This ensured downstream compatibility with serum. The MIP amount was also fixed in the on-line MIPs. Single MIP columns (Paper II and Paper III) contained $6 \mathrm{mg}$ of polymer and the mix MIP columns (Paper III) contained were $3 \mathrm{mg}$ per column. As a consequence, the total binding capacity is fixed in on-line MISPE.

The first step in on-line MISPE is to find the optimal loading mobile phase. An ideal loading mobile phase should retain the target molecule with selectivity. To determine an optimal loading mobile phase for the ELPLYR-MIPs (ELP-MIP 1 and ELP-MIP 2) in Paper II, the retention factor was the ideal parameter. By coupling the MIPs (and NIPs) directly to the MS, retention factors can be calculated by determining the retention time, flow rate, and dead volume. The higher the retention factor, the stronger the retention of ELPLYR. However as discussed with the mMIPs, selectivity is the determining factor in the potential 
compatibility with complex matrices. Selectivity can be determined by the relative retention factors of the ELP-MIPs and their corresponding NIPs.

On-line MIPs targeting NLLGLIEAK found the optimal isocratic loading mobile phase to be $100 \% 20 \mathrm{mM}$ FA. ${ }^{120}$ The ELP-MIPs were coupled to the MS and $2 \mu \mathrm{L}$ of ELPLYR ( $5 \mathrm{nM}$ ) was injected onto the column. When the loading mobile phase was $100 \%$ FA, the $\mathrm{k}$ value could not be determined. This was due to the ELPLYR not eluting from either ELP-MIP 1 or 2 at the tested flow rates $(20,30$, and $40 \mu \mathrm{L} / \mathrm{min}$ This is possibly due to secondary interaction effects between the MIP and analytes in the presence of the aqueous phase and strong hydrophobic interactions. ELPLYR could be eluted off the column when washed with high organic (i.e. $\geq 50: 50 \mathrm{MeCN}: \mathrm{H}_{2} \mathrm{O}$ ) as seen with the mMIPs. While the strong interactions between ELPLYR and the MIPs are ideal, there is no observable selectivity. The NIPS also retained ELPLYR which suggests the binding mechanism is strongly non-specific or the target peptide is trapped in the polymer matrix. The ELP-MIPs are not core-shell MIPs but rather fully porous polymers. There are likely to be more binding sites deep within the 3D polymer network. Therefore, some organic solvent should be incorporated in the loading mobile phases to enhance the specific binding. The observable trend was a reduction of $\mathrm{k}$ with increasing MeCN. However, ELP-MIP 1 was found to have the highest retention factors between 3 and $5 \% \mathrm{MeCN}$, and importantly, had higher retention factors compared to its NIP. Further finetuning at 1, 2, 3, and 4\% MeCN found the greatest selectivity at 97:3 20 mM FA:MeCN (Fig. 12). ELPYR did not elute off from ELP-MIP 1 and ELP-NIP 1 at 99:1 and 98:2 20 mM FA:MeCN suggesting non-specific interactions remain with $1-2 \% \mathrm{MeCN}$. 


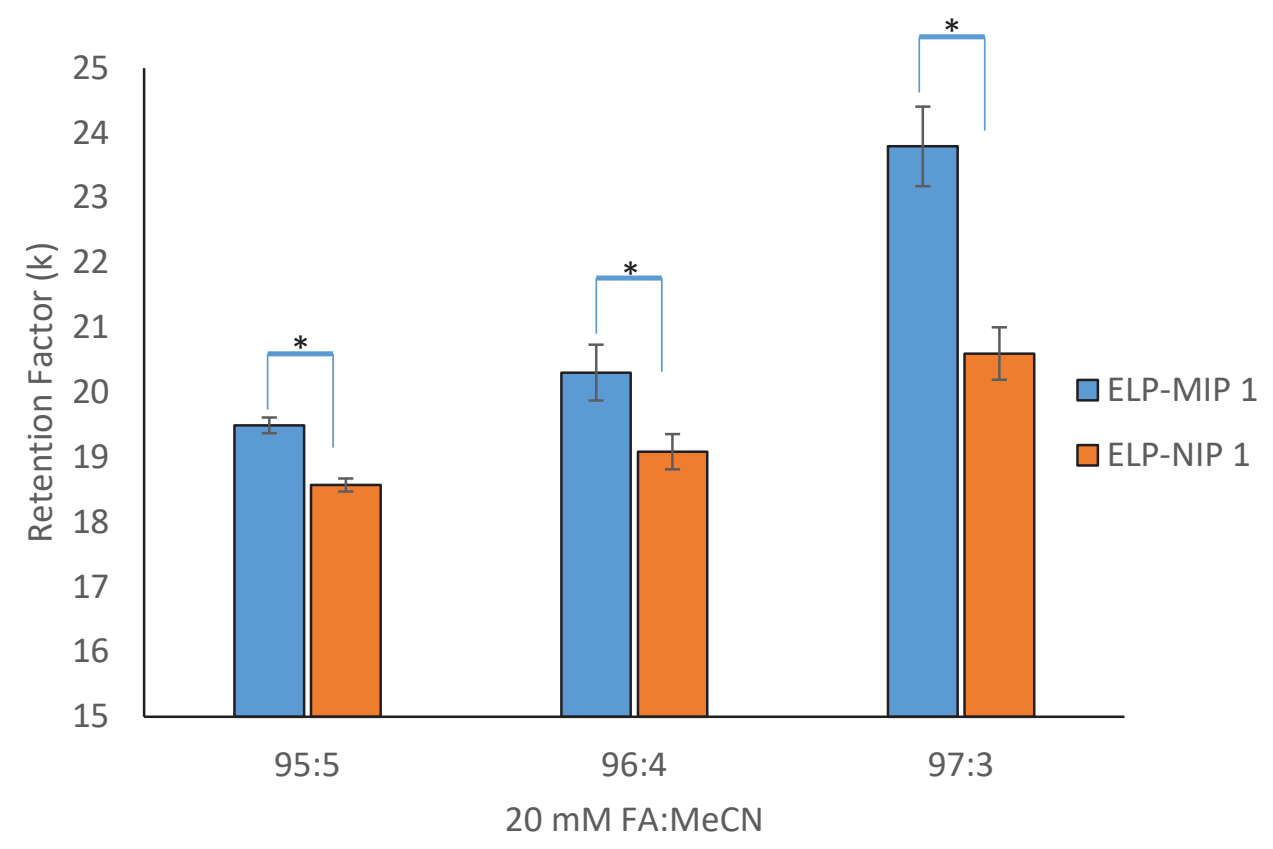

Figure 12. Retention factors of MIP 1 and NIP 1 at $20 \mu \mathrm{L} \mathrm{min}{ }^{-1}$ isocratic flow with mobile phases 95:5, 96:4 and 97:3 mM FA:MeCN (v/v). *P $\leq 0.05$ Reproduced with permission from Fig. 3 paper II. Copyright (C) 2020, Elsevier.

\section{Extraction Time}

As with the mMIPs, extraction time in on-line MISPE is crucial for ensuring higher throughput without loss of the target analyte. Unlike the mMIPs, the extraction step allows for the matrix components to be discarded, acting as a wash step. Therefore, extraction time must be long enough to allow for the matrix components to pass through the MIP while also retaining the target peptide. ELP-MIP 1 retains ELPLYR strongly as there was no significant loss of peptide after $20 \mathrm{~min}$, which equates to approximately 40 column volumes. However, the extraction time should allow for non-specific peptides to be washed off the MIP. This was investigated using digested BSA and extraction for 5, 10, and 20 minutes and determining the retention of peptides at each time point. Many of the BSA peptides had a strong affinity for ELP-MIP 1. BSA peptides have previously been shown to have a strong affinity in on-line MISPE ${ }^{161}$. There were only subtle differences between each time point with $66.5 \pm 39.5 \%, 59.3 \pm 6.3$, and $51.9 \pm 21.6$ of the BSA peptides retained following 5, 10, and $20 \mathrm{~min}$ extractions. Therefore, $10 \mathrm{~min}$ was selected as the extraction time to balance throughput with the removal of non-specific peptides. 


\section{Duplex Extractions}

In Paper III, ELP-MIP 1 was used in combination with the NLLGLIEAK MIP (NLL-MIP) for duplex extraction and analysis of ProGRP and NSE. As such the loading mobile phase needed to be again optimised to ensure selective capture of both NLLGLIEAK and ELPLYR. As discussed above, the NLL-MIP had been successfully optimised for serum extractions of NLLGLIEAK using 20 mM FA. However, the ELP-MIP has no selectivity at $100 \%$ FA. Therefore, a compromise mobile phase was needed to bridge the difference. ELP-MIP 1 and the NLL-MIP have different FMs (TVU for ELP-MIP 1 and EAMA.HCl for the NLL-MIP). Therefore, the affinity for their respective target peptides may vary in certain loading mobile phases.

The first step was to determine if the NLL-MIP could tolerate the optimal loading phase of ELP-MIP 1 (i.e. 97:3 20 mM FA:MeCN). Interestingly, the NLL-MIP had poor affinity towards NLLGLIEAK 97:3 $20 \mathrm{mM}$ FA:MeCN with an extraction recovery of $27.9 \pm 4.4 \%$. This is likely due to the EAMA.HCl FM, as similar losses were observed with mMIP C during washing with 3-10\% MeCN. This presents a challenge as loading mobile phases of $\leq 2 \% \mathrm{MeCN}$ are more likely to induce have non-specific interactions. The selectivity and specificity of ELP-MIP 1 and the NLL-MIP were assessed by comparing the recoveries of the specific peptide and the non-specific peptide at 100:0, 99:1, and 98:2 20 mM FA:MeCN. For ELP-MIP 1, the specificity was poor with no observable difference in the recoveries of NLLGLIEAK and ELPLYR. Conversely, the NLL-MIP demonstrated specificity with increasing MeCN. At 98:2 20 mM FA, the NLL-MIP had an extraction recovery of NLLGIEAK is approximately 100\%, the ELPLYR was poorly retained with an extraction recovery of approximately $5 \%$.

Selectivity was also evaluated by comparing the extraction recoveries of the target peptides by the MIPs and their NIPs at 98:2 $20 \mathrm{mM} \mathrm{FA:MeCN}$. The trend is completely reversed for specificity, with ELP-MIP demonstrating good selectivity. The extraction recovery of the target peptide (ELPLYR) was $108.0 \pm 4.4 \%$. Conversely, the ELP-NIP had a poor extraction recovery of $10.5 \pm 0.7 \%$ (Fig. 13). The NLL-MIP conversely saw no observable difference in the extraction recovery between the MIP and NIP. As such, 98:2 $20 \mathrm{mM}$ was selected as the loading mobile phase moving forward. 


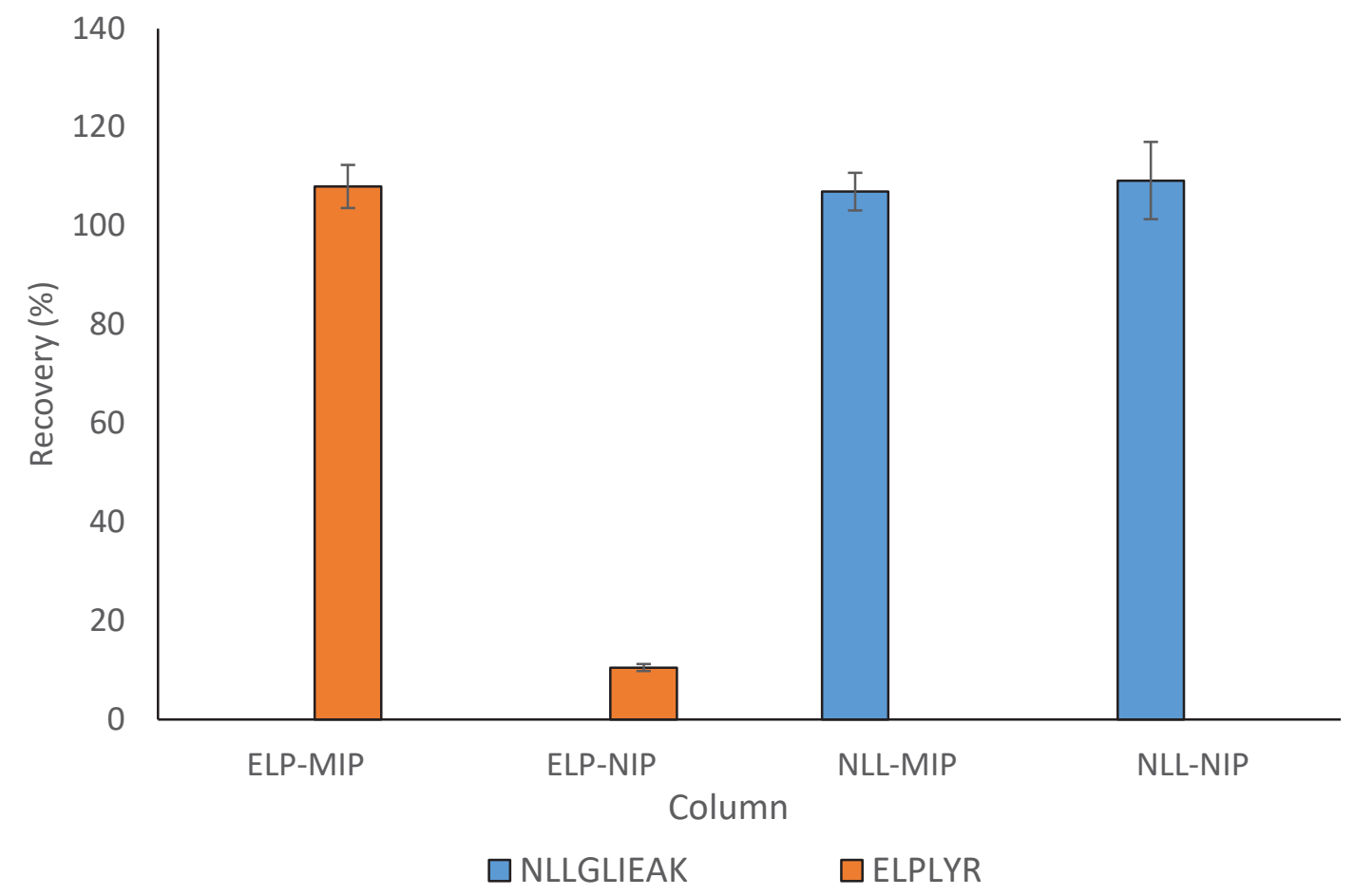

Figure 13: The recoveries of ELPLYR (10 nM) and NLLGLIEAK (10 nM) in $50 \mathrm{mM} \mathrm{ABC}$ after extraction by single columns (NLL-MIP, ELP-MIP, NLL-NIP, and ELP-NIP $(n=3)$. 


\subsection{MISPE Method Evaluation}

Following method development, the MISPE methods were evaluated to determine the viability of the MIPs in the extraction of SCLC biomarkers in complex matrices.

\subsubsection{Magnetic SPE Method Evaluation}

In Paper I, maximum binding capacity and affinity were used to evaluate the magnetic SPE method. This was determined by using binding isotherms. The non-linear shape of the MMIP curve (Fig. 14) suggested a mechanism of selective binding by NLLGLIEAK via the molecularly imprinted binding sites in the mMIP. Conversely, mNIP C has a linear binding mode, suggesting non-selective binding. This confirms that the extraction conditions are ideal for selective binding and MMIP C is likely to discriminate NLLGLIEAK in more complex matrices. The maximum binding capacity of mMIP C was calculated to be $7.4 \mathrm{pmol}$ NLLGLIEAK/mg mMIP. The dissociation constant $\left(K_{d}\right)$ for mMIP $C$ was calculated to be $2.18 \times 10^{-9} \mathrm{M}$. This suggests that mMIP C-NLLGLIEAK binding affinity is comparable in strength to antibody-antigen interactions. As such, it can be presumed that the imprinting of the template was highly successful, and the generated cavities are specific with high affinity for NLLGLIEAK. High affinity will undoubtedly aid the mMIP in complex matrix extractions. One of the key aims of the present work was to evaluate the various MIP strategies. It is clear the post-magnetisation strategy of mMIP C using EAMA. $\mathrm{HCl}$ as the FM produces a functional MIP. 


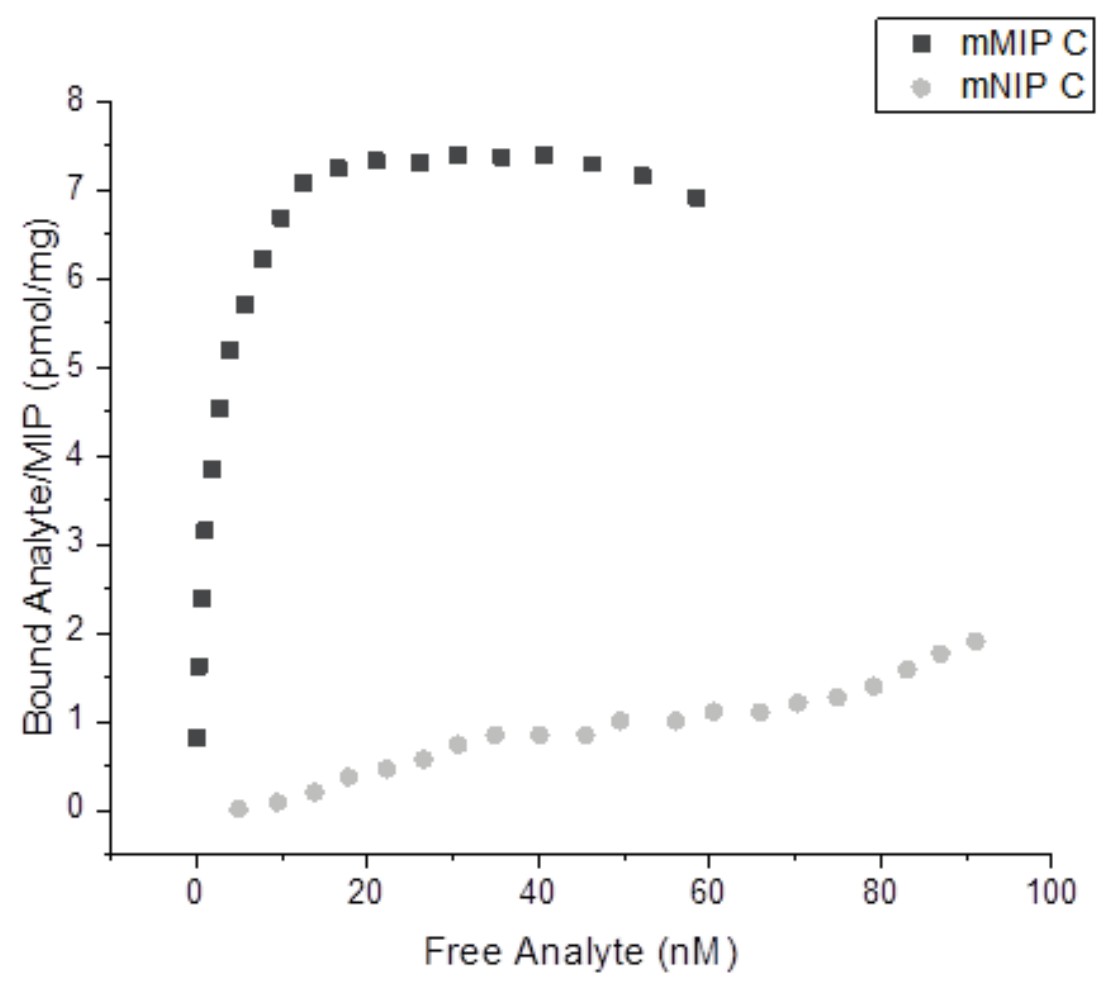

Figure 14: Binding isotherms for mMIP $C$ and $\mathrm{mNIP} C$, expressed as bound analyte/mg mMIP or mNIP vs. concentration of free analyte. Samples consisted of NLLGLIEAK IS (5 nM) in ABC (50 mM) containing 10 nM digested BSA ( $n=2)$. Reproduced with permission from Fig. 2 paper $I$.

The final magnetic SPE method was evaluated by looking at the affinity of mMIP C towards other peptides; specifically, other signature peptides of ProGRP isoform 1 (i.e. ALGNQQPSWDSEDSSNFK and LSAPGSQR) and NLLGLIEAK at each step of the extraction (extraction, wash, and elution) in a simple matrix (i.e. 50 $\mathrm{mM} A B C)$. This provides insights into the expected behaviour in more complex matrices. The final method determined that mMIP $C$ had an affinity for NLLGLIEAK and ALGNQQPSWDSEDSSNFK, with $>85 \%$ of each peptide retained on MMIP C following extraction (Fig. 15). LSAPGSQR did not bind well to MIP C, with 75.6 $\pm 10.6 \%$ remaining unbound. The wash step demonstrated modest losses for each of the peptides. The elution step had an interesting result, with only NLLGLIEAK eluting from the MIP. This suggests a selective elution, whereby only specific peptides can be released into the eluent. This generates a clean extract that is essential for MS/MS analysis. 


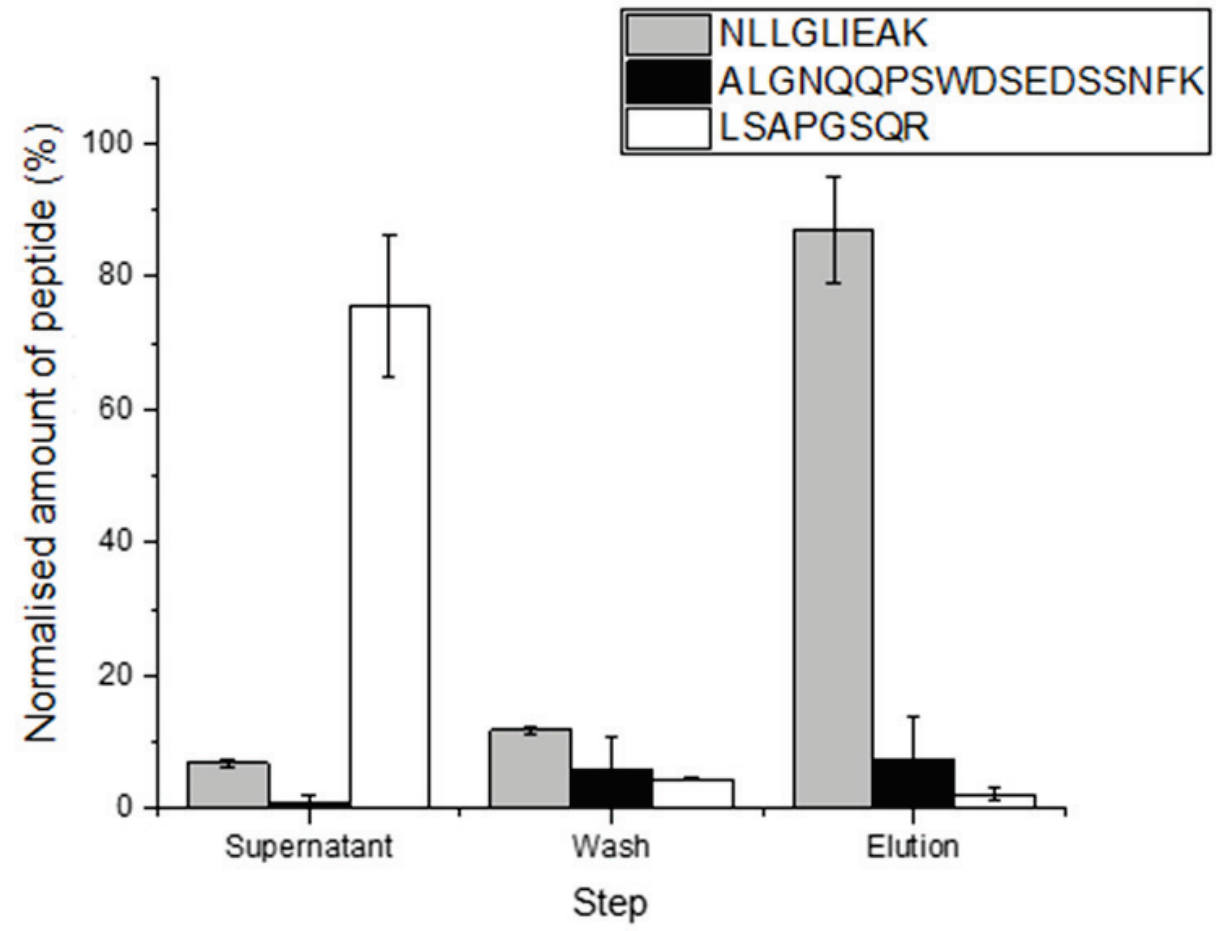

Figure 15: Evaluation of the selectivity of each step in the magnetic SPE method using digested ProGRP, as represented by the normalised amount of peptide (\%) \pm standard deviation of three ProGRP peptides for the three steps. Samples consisted of ProGRP (182 nM) and NLLGLIEAK IS (5 nM) in ABC (50 mM) containing 10 nM digested BSA. Samples were extracted for 5 min., washed in $50 \mathrm{mM} \mathrm{ABC}(100 \mu \mathrm{L})$ for $5 \mathrm{~min}$. and eluted with 80:15:5 $\mathrm{MeCN}: \mathrm{H}_{2} \mathrm{O}$ :FA for 5 min. ( $\left.n=3\right)$. Reproduced with permission from Fig. 5 paper I.

\subsubsection{On-line MISPE Method Evaluation}

The on-line MISPE was evaluated in paper // by assessing both the linearity of the on-line MISPE extraction by ELP-MIP 1 in a simple matrix (i.e. $50 \mathrm{mM} A B C$ ) and by comparing the relative recoveries of digested NSE spiked into matrices of various complexity. This provides an insight into the downstream compatibility of ELP-MIP 1 extractions of complex matrices. Linearity was determined by injecting digested NSE in the range $10-100 \mathrm{ng} / \mathrm{mL}$ in $50 \mathrm{mM}$ ABC containing a $100 \mathrm{nM}$ digested $\mathrm{BSA}(\mathrm{n}=3)$ onto the MIP column. Linear regression was obtained by plotting the intensity of ELPLYR vs. the added concentration and the curve was weighted at $1 / x$. The correlation value $\left(R^{2}=0.99\right)$ was within acceptable limits and higher than for previously reported for the NLL-MIP $\left(R^{2}=0.98\right)^{120}$.

Prior evaluation of the performance of ELP-MIP 1 in more complex matrices was essential as it had a relatively low imprinting factor (IF, see section 3.3.5). Therefore, the selectivity of the on-line MISPE may 
be lower than other applications (i.e. magnetic SPE). The evaluation was performed on digested NSE spiked into three different matrices consisting of $50 \mathrm{mM} \mathrm{ABC}$ containing digested $\mathrm{BSA}$, serum pre-treated by offline SPE (in-house C18 cartridges), and untreated digested serum. The extraction recovery of ELPLYR was $100.4 \pm 5.0 \%$ in the simple matrix (i.e. $50 \mathrm{mM} \mathrm{ABC}$ containing digested BSA at 10-fold molar amount. The extraction recovery from the SPE treated serum was excellent at $100.8 \pm 6.2 \%$ and comparable to those in a simple matrix. MIP extractions from digested serum, on the other hand, showed a lower recovery at $61.6 \pm 1.9 \%$. This suggests ELP-MIP 1 possibly has good selectivity. This can be presumed due to the extraction of ELPLYR from the matrix with non-specific peptides in concentrations many orders of magnitude higher than ELPLYR. As such, selectivity must play a role as the MIP capacities are often low. This is interesting as the IF of ELP-MIP 1 is low under the on-line MISPE conditions. This illustrates the challenges with using IF as a predictor of selectivity. 


\subsubsection{On-line Duplex MISPE Method Evaluation}

In Paper III, the duplex on-line MISPE was evaluated in two formats: MIPs in series (i.e. two columns connected during extraction) and mixture columns containing a 50:50 mix of each MIP (Fig. 16)
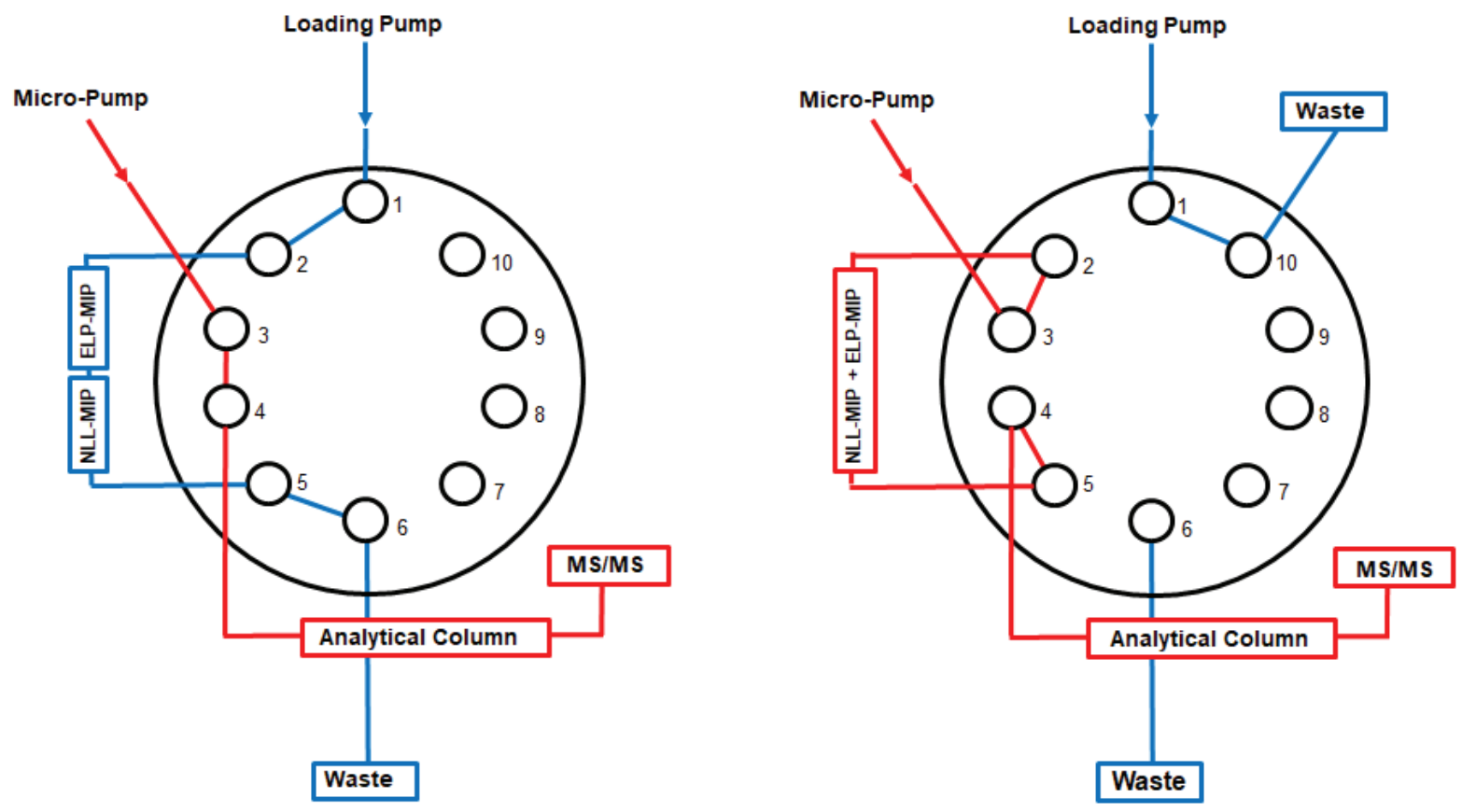

Figure 16: Schematic representation of the MISPE duplex formats. The MIPs can be attached either in series (ELPMIP and NLL-MIP columns ( $5 \mathrm{~mm} \times 1.5 \mathrm{~mm}$ ID) connected by HPLC tubing) or as an intimate 1:1 mixture of the two MIPs in a single column ( $5 \mathrm{~mm} \times 1.5 \mathrm{~mm}$ ID).

A key parameter to determine the optimal duplex format is the extraction recovery of the two target peptides spiked into both a simple matrix (i.e. $50 \mathrm{mM} \mathrm{ABC}$ containing digested BSA) and digested serum (Fig. 17). In the simple matrix, all MIPs had high extraction recoveries of both NLLGLIEAK and ELPLYR. Therefore, two MIPs in conjunction can retain two unique targets.

The series MIPs were tested in both column orders (i.e., ELP-MIP then NLL-MIP, and NLL-MIP then ELPMIP) to ascertain the effects of column order on extraction. The target peptides are not affected by column order as there was no significant difference between either configurations. The recoveries of the series MIPs were between $95-107 \%$. The series NIPs, conversely, were comparable to the single NIP columns. The NIPs had strong, non-specific interactions for NLLGLIEAK and poor affinity towards ELPLYR. The NLL-ELP NIP series had extraction recoveries of $87.9 \pm 10.2 \%$ and $6.3 \pm 1.5 \%$ for NLLGLIEAK and ELPLYR, respectively. As seen with the series MIPs, the order of the NIPs did not affect performance with ELP-NLL 
NIP series had recoveries of $90.6 \pm 11.2 \%$ and $5.4 \pm 2.8 \%$ for NLLGLIEAK and ELPLYR, respectively. The mix NIPs had extraction recoveries of $116.8 \pm 3.8 \%$ and $4.2 \pm 0.7 \%$ for NLLGLIEAK and ELPLYR, respectively. This is comparable to the performance of the single NIPs. The non-specific interactions of NLLGLIEAK obfuscate the selectivity of duplex MISPE in simple matrices due to an abundance of unsaturated, non-selective binding sites. Therefore, the non-specific sites must be saturated to determine the true selectivity, and therefore, the viability of the duplex formats.

Extraction of ELPLYR and NLLGLIEAK spiked into digested serum allows for accurate determination of the optimal duplex formats. As the non-specific sites are saturated by exceedingly high concentrations of tryptic peptides and other matrix components, allowing for saturation of non-specific binding sites. Therefore, the primary binding mechanism is specific binding. The 1:1 mix MIPs had extraction recoveries of both peptides with extraction recoveries of NLLGLIEAK and ELPLYR of $15.7 \pm 2.3$ and $19.2 \pm 8.4 \%$, respectively. This represents extraction recoveries approximately $40 \%$ lower than simple matrix extractions. The significant loss in extraction recovery is likely due to limited binding capacity as the mix MIPs contain only $3 \mathrm{mg}$ of each MIP. There is clear selectivity as the MIPs still have higher extraction recoveries compared to the $1: 1 \mathrm{mix}$ NIP. The $1: 1 \mathrm{mix}$ NIP had extraction recoveries of $5.63 \pm 1.7 \%$ and $5.00 \pm 2.8 \%$ for NLLGLIEAK and ELPLYR, respectively.

The series MIPs, on the other hand, have $6 \mathrm{mg}$ of each MIP and as such should have a much higher capacity. Extraction recoveries of the NLL-ELP MIP series were $85.8 \pm 3.8 \%$ and $49.1 \pm 6.7 \%$ for NLLGLIEAK and ELPLYR, respectively, whereas the ELP-NLL MIP series had extraction recoveries of $86.5 \pm 0.9 \%$ and $50.8 \pm \% 11.6$ for NLLGLIEAK and ELPLYR, respectively. Compared to the 1:1 mix MIP, extraction recoveries increased approximately 5.5-fold and 2.6-fold for NLLGLIEAK and ELPLYR, respectively. These increases in extraction recoveries could be attributed to the 2-fold increase in the mass of each MIP (i.e., enhanced capacity) and column volume.

The initial series extractions had acceptable recoveries, however, when evaluating the intra- and interday variability over eight days of serum extractions, considerable variation was observed. The intraday variability was similarly high for both peptides. Extraction recoveries of NLLGLIEAK varied between 0.620.6\% RSD (median RSD of 13.85\%). Similarly, ELPLYR had RSDs ranging from 6.7-32.4\% (median RSD of $18.5 \%)$. It is important to note there was no IS correction. Interday recoveries were $65.7 \pm 21.0 \%$ (RSD=32.0\%) and 37.5 $\pm 12.9 \%(R S D=34.4 \%)$ for NLLGLIEAK and ELPLYR, respectively. Continuous extractions in complex matrices (e.g. serum) are likely to interfere with the specific binding. As such, the MIPs may become less selective the target peptides may be more likely to be lost during extraction. 
Therefore, the on-line MISPE needs further optimisation to minimise the effects of tryptic peptides of high abundant proteins.
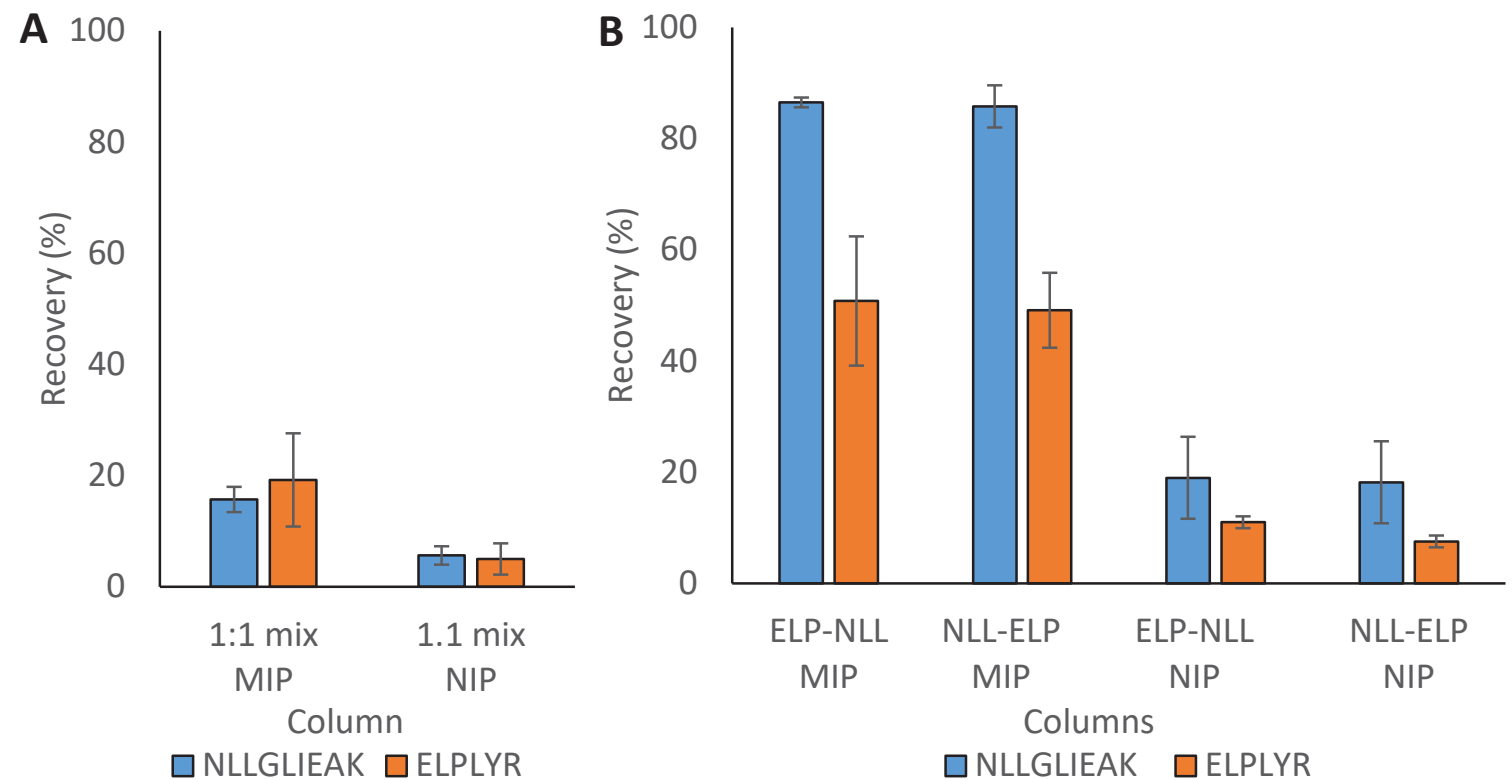

Figure 17: Duplex analysis of NLLGLIEAK and ELPLYR in digested human serum. (A) The recoveries of ELPLYR (10 nM) and NLLGLIEAK (10 nM) in digested human serum after extraction by a 1:1 mixture of both MIPs and NIPs $(n=3)$. (B) The recoveries of ELPLYR (10 $\mathrm{nM})$ and NLLGLIEAK (10 nM) digested human serum after extraction by MIPs (and NIPs) connected in series (both orders, $n=3$ ).

\subsubsection{On-line MISPE Optimisation}

The extraction method was optimised by reducing the extraction time from $10 \mathrm{~min}$ to $5 \mathrm{~min}$, and the valve switching protocol was changed to prevent non-specific peptides retained in the MIPs from eluting onto the analytical column. The intra- and interday precision of extraction recovery in serum over 5 days were used to assess the efficacy of method optimisation (Table 8). The intraday variation of NLLGLIEAK recovery ranged from 5.3-23.6\% RSD (median of 21.1\%). ELPLYR similarly had intraday RSDs ranging from 8.5-22.9\% (median of $11.8 \%$ ). The interday recovery of both peptides was increased to $82.0 \pm 10.0 \%$ and $64.9 \pm 9.5 \%$ for NLLGLIEAK and ELPLYR, respectively. The extraction optimisation improved the interday variability of both NLLGLIEAK (RSD=12.2\%) and ELPLYR (interday RSD=14.6\%) compared to $32.0 \%$ and $34.4 \%$, for NLLGLIEAK and ELPLYR, respectively for the non-optimized extraction protocol. 
Table 8: The intra-and interday recoveries of NLLGLIEAK and ELPLYR after extraction by the NLL-ELP MIP series using the optimised extraction method over 5 days.

\begin{tabular}{ccccc} 
& \multicolumn{2}{c}{ NLLGLIEAK } & \multicolumn{2}{c}{ ELPLYR } \\
& Recovery (\%) & RSD (\%) & Recovery (\%) & RSD (\%) \\
\hline Day 1 & 94.1 & 5.3 & 60.4 & 9.9 \\
Day 2 & 86.3 & 23.4 & 54.3 & 22.9 \\
Day 3 & 67.8 & 23.6 & 61.2 & 16.8 \\
Day 4 & 76.7 & 21.1 & 70.5 & 8.5 \\
Day 5 & 84.9 & 6.9 & 78.4 & 11.8 \\
\hline Interday & 82.0 & 12.2 & 64.9 & 14.6 \\
\hline
\end{tabular}

\subsubsection{Imprinting Factors}

MIP selectivity is often quantified by imprinting factor (IF), a determination of the efficiency of a molecular imprinting process. This is achieved by directly comparing the binding of the target analyte to the MIP to the binding of the same analyte to the NIP. IF is a highly variable measurement and highly dependent on experimental conditions. This is due to the myriad factors, including the balance of selective and nonselective binding to the MIP under the conditions of the measurement. Therefore, careful method development is essential. Higher IF values indicate that there are conditions under which selective binding of an analyte to a MIP can be realised and potentially exploited. In Paper I, the binding isotherm was used to calculate an IF of 6.1 for mMIP C, which suggests that molecular imprinting was successful and that ideal binding conditions have been established under which NLLGLIEAK could be extracted selectively from an aqueous matrix. This is comparable to other mMIPs targeting proteins and peptides. MIPs targeting angiotensin I, angiotensin II, and human serum transferrin (HTR) were reported to have IFs of $4.9,5.2$, and 5.49, respectively ${ }^{128,134}$. IF is a good indicator of imprinting efficiency ${ }^{160}$, therefore, the higher the IF the more likely a MIP will have good selectivity in complex matrices.

Binding isotherms pose a practical challenge in on-line MISPE due to the coupling to the MS. Therefore, in Paper II, IF was calculated using retention factors. Loading with 97:3 $20 \mathrm{mM} \mathrm{FA:MeCN} \mathrm{(v/v)} \mathrm{gave} \mathrm{the}$ highest $k$ value for ELP-MIP 1 and represented an IF of 1.2. This is considerably lower when compared to mMIP C. However, as discussed above, IF is highly variable and it is difficult to accurately compare the IFs 
of different MISPE methods. When compared to other on-line MIPs, it is slightly higher than the previously reported IF for the NLL-MIP which had an imprinting factor of $1.1^{120}$.

\subsubsection{Comparisons of the Single and Duplex on-line MISPE Approaches}

The recoveries of ELPLYR and NLLGLIEAK are improved in the duplex extractions. The extraction recovery of ELPLYR was similar for single- and duplex. The singleplex extraction recovery was 60\% (Paper II) and duplex extraction recovery was $65 \%$ (Paper III). The duplex extraction recovery represented only a modest increase. It is important to note that unlike the singleplex method, the duplex extraction conditions (i.e. loading mobile phase) were not optimal for ELPLYR selectivity by ELP-MIP 1. The singleplex extraction recovery of NLLGLIEAK by the NLL-MIP was previously reported to be approximately $25 \%{ }^{120}$. The duplex extraction recoveries increased over 3 -fold to $82 \%$. This is likely due to the improved selectivity of the NLL-MIP in the loading mobile phase of the duplex method. As discussed previously, selectivity is often the best indicator of extraction performance. Importantly, the throughput is more than double as both analytes are measured in a single analysis, and no MIP column changes are required. Therefore, analytical performance is demonstrably improved by using the duplex method. 


\subsection{Analytical Performance in Complex Matrices}

A primary goal of the present work was to assess the analytical performance of all the MIPs in human serum. The method development and optimisation aimed to produce a MISPE protocol for selective capture of the signature peptides of the SCLC biomarkers.

\subsection{Linearity and Accuracy of On-line MISPE}

Linearity was assessed in Paper II and III and method accuracy was calculated in Paper III. The disease range of SCLC ranges from low $\mathrm{pM}$ to $\mathrm{nM}$. Therefore, for a MISPE to be clinically viable, it must be able to extract and quantify ProGRP and NSE in this range. This can be assessed by the linearity of the extractions in this range.

ELP-MIP 1 was linear in the disease range $(0.3-30 \mathrm{pM})$ in both the single and duplex formats, with a coefficient of determination $\left(\mathrm{R}^{2}\right)$ value of 0.99 for both methods. The NLL-MIP showed similar linearity with an $R^{2}$ of 0.98 . This is the same as the previously reported singleplex extractions for the NLL-MIP.

The accuracy of the duplex method was evaluated by using the developed linear calibration graphs and plotting the signals from two serum sample extractions: one containing $0.3 \mathrm{nM}$ ELPLYR and $30 \mathrm{nM}$ NLLGLIEAK and another containing $30 \mathrm{nM}$ ELPLYR and $0.3 \mathrm{nM}$ NLLGLIEAK. The accuracy was excellent at the high concentration ( $30 \mathrm{nM}$ ) with NSE and ProGRP had accuracies of $97.3 \pm 12.8$ and $108.1 \pm 7.8$, respectively. Conversely, the accuracy was adequate at the low concentration levels $(0.3 \mathrm{nM})$, but with high variability with accuracies for NSE and ProGRP of $87.3 \pm 41.4$ and $85.8 \pm 50.2$, respectively. The adequate accuracy at the low concentration is likely due to the low signal to noise $(\mathrm{S} / \mathrm{N})$ ratio. This may be attributed to the myriad non-specific peptides being present in the analysis.

\subsubsection{Extraction Recovery, Limits of Detection and Limits of Quantification}

Extraction recovery, the limit of detection, the limit of quantification were assessed from complex matrices in Paper I, II, and III (Table 9). For effective clinical analysis, the MISPE protocols must be able to distinguish between patients who are healthy and those in a disease state. This can be achieved with the analytical sensitivity to quantify the low biomarker levels present in healthy patients (i.e. the reference level). ELP-MIP 1 was very successful in extracting ELPLYR from serum with good sensitivity. The singleplex approach recovered approximately 60\% of ELPLYR from spiked serum with a LOD of $46 \mathrm{pM}$ from a 0.26 $\mathrm{nM}$ sample. The duplex approach was similarly sensitive with a recovery of $65 \%$ and a LOD of $110 \mathrm{pM}$. Both approaches can detect NSE below the reported reference level in humans which is approximately $220 \mathrm{pM}^{156}$. The LOQs for the singleplex and duplex approach were 210 and $370 \mathrm{pM}$, respectively. Interestingly, only the singleplex approach gives a LOQ under the reference level. Therefore, only the 
singleplex approach could be clinically viable under the present conditions. The disparity in detection limits between the single and duplex approaches is likely due to extraction conditions. As discussed previously, selectivity is an essential parameter in MISPE. ELP-MIP 1 is most selective at 97:3 $20 \mathrm{mM}$ FA:MeCN, the conditions used in the singleplex extractions. Conversely, the duplex extractions use 98:2 20 mM FA:MeCN to accommodate the NLL-MIP. The sample preparation was similar between the two approaches with digested human serum used as the sample matrix.

The NLL-MIP had slightly better analytical performance than ELP-MIP 1 in the duplex MISPE. The NLL-MIP had an extraction recovery of $82 \%$ with a LOD and LOQ of 60 pM and 200 pM, respectively. However, both the LOD and LOQ are above the reference level for ProGRP in humans (i.e. $7.6 \mathrm{pM})^{152}$. The LOD is higher than previously reported for the NLL-MIP, which achieved a LOD of $11 \mathrm{pM}$. However, the sample preparation is not comparable with the approach used for the NLL-MIP in the present study. Interestingly, mMIP C had the most sensitive MISPE method with a LOD and LOQ for ProGRP of 39 pM and 129 pM, respectively. This is despite an extraction recovery of only $25 \%$. This sensitivity could be attributed to the selective elution step. The LOD and LOQ are calculated using the $\mathrm{S} / \mathrm{N}$ ratio of blank serum extracts with the spiked serum extracts. Therefore, to generate a better LOD and LOQ, it is essential to have clean extracts. The on-line MISPE can retain more of the target analyte, but there is far less control of the removal of non-specific peptides (due to the coupling to the MS). This could account for the higher extraction recoveries but poorer limits of detection as the non-specific peptides retained on the MIP give a noisier background. The present study used an older MS instrument. With more modern, sensitive MS, it is highly likely that the detection limits would be lower and possibly below the reference level for all the MIPs used in the present study.

Table 9. The recovery, LOD, and LOQ of the MISPE methods used in the present study

\begin{tabular}{ccccccc}
\hline MIP & Biomarker & MISPE method & $\begin{array}{c}\text { Recovery } \\
(\%)\end{array}$ & $\begin{array}{c}\text { LOD } \\
(\mathrm{pM})\end{array}$ & $\begin{array}{c}\text { LOQ } \\
(\mathrm{pM})\end{array}$ & Paper \\
\hline mMIP C & ProGRP & magnetic SPE & $25 \%$ & 39 & 129 & $I$ \\
NLL-MIP & ProGRP & duplex on-line MISPE & $82 \%$ & 60 & 200 & III \\
ELP-MIP 1 & NSE & duplex on-line MISPE & $65 \%$ & $110^{*}$ & 370 & III \\
ELP-MIP 1 & NSE & Singleplex on-line MISPE & $60 \%$ & $46 *$ & $210^{*}$ & \multicolumn{1}{|c}{} \\
\hline
\end{tabular}




\section{Concluding Remarks and Future Perspectives}

The present thesis was a highly multidisciplinary study in polymer, protein, and bioanalytical chemistry. The present work has built a foundation on which MIP synthesis and analytical strategies can be enhanced to improve selectivity and analytical performance. With careful development of novel MISPE protocols, the future directions for MIP design and application have been identified. The challenges of MISPE have been made clearer and knowledge on method development has been obtained.

The magnetic SPE by mMIPs has allowed for the selective capture of NLLGLIEAK and the quantification of ProGRP at low levels. The mMIP demonstrated that selectivity is a more essential parameter in determining MISPE analytical performance. The magnetic SPE protocol provided insights into the advantages of off-line MISPE. This was achieved by the development of a selective elution that yielded clean extracts for analysis, enhancing the analytical performance. Furthermore, the optimal mMIP format was elucidated; namely, core-shell mMIPs. This added to the body of knowledge and will aid in further mMIP improvements in the future.

The novel ELP-MIPs aided in the development of MIP based strategies for SCLC biomarker analysis. These MIPs provided insights into synthetic strategies for enhancing selectivity. The ELP-MIPs were able to selectively capture ELPLYR from complex matrices with high recoveries and modest selectivity. The ELPMIPs had sufficient analytical performance with a LOQ below the reference limit in human serum. This represents one of the few MIPs that have clinical potential reported in the literature.

The development of the duplex MISPE protocol represents a significant step in the direction of clinically viable MIPs. The two MIPs in combination were able to maintain an affinity for both signature peptides although with acceptable selectivity. The duplex MISPE protocol was able to successfully quantify NSE and ProGRP in the disease range in a linear fashion. Improvements in selectivity will enhance the analytical performance of the duplex MISPE protocol. The development of a MIP based duplex analysis of ProGRP and NSE represents an improvement to conventional routine measurements (e.g. singleplex immunoassays).

The present thesis has contributed to the knowledge of MISPE and has laid a platform for future MIP improvements and applications.

While MIPs have been developed for the extraction of proteins and peptides for nearly 30 years, novel materials that function in complex matrices such as serum have only recently become more commonly 
reported. Strategies for overcoming the selectivity challenges are being implemented and MISPE methods have begun to see detection limits in the low pM range. As such, it likely that many more MIPs targeting novel proteins will be developed in the future.

MIP selectivity must be further improved to enhance the extractions of low-abundant biomarkers and generate limits of detection below reference levels. The automation of MISPE is a step forward in developing user-friendly protocols with high throughput. The duplex MISPE in the present work represents a significant development in MIP-based applications. The two MIPs demonstrate successful duplex analysis of two low abundant biomarkers in human serum. However, at present, the detection limits are not below the reference level, and therefore, in its current state, the duplex MISPE protocol is not clinically viable. Improvements in the form of enhanced selectivity are needed.

If the selectivity improvements can be achieved, more MIPs will likely become commercially available and potentially clinically viable for use in protein and peptide analyses. 


\section{References}

1. Fischer, E., Einfluss der Configuration auf die Wirkung der Enzyme. Berichte Der Deutschen Chemischen Gesellschaft 1894, 27 (3), 2985-2993.

2. Haupt, K., Biomaterials: Plastic antibodies. Nature Materials 2010, 9 (8), 612-614.

3. Polyakov, M. V., Adsorption properties and structure of silica gel. Zhurnal Fizicheskoi Khimii 1931, 2, 799-805.

4. Wulff, G.; Sarhan, A.; Zabrocki, K., Enzyme-analogue built polymers and their use for the resolution of racemates. Tetrahedron Letters 1973, 14 (44), 4329-4332.

5. Andersson, L.; Sellergren, B.; Mosbach, K., Imprinting of amino acid derivatives in macroporous polymers. Tetrahedron Letters 1984, 25 (45), 5211-5214.

6. Arshady, R.; Mosbach, K., Synthesis of substrate-selective polymers by host-guest polymerization. Die Makromolekulare Chemie: Macromolecular Chemistry and Physics 1981, 182 (2), 687-692.

7. Haupt, K., Molecularly Imprinted Polymers: The Next Generation. Analytical Chemistry 2003, 75 (17), 376 A-383 A.

8. Owens, P. K.; Karlsson, L.; Lutz, E. S. M.; Andersson, L. I., Molecular imprinting for bio-and pharmaceutical analysis. Trends in Analytical Chemistry 1999, 18 (3), 146-154.

9. Haupt, K.; Mosbach, K., Molecularly imprinted polymers and their use in biomimetic sensors. Chemical Reviews 2000, 100 (7), 2495-2504.

10. Kempe, M.; Mosbach, K., Separation of amino acids, peptides and proteins on molecularly imprinted stationary phases. Journal of Chromatography A 1995, 691 (1-2), 317-323.

11. Rachkov, A.; Minoura, N., Recognition of oxytocin and oxytocin-related peptides in aqueous media using a molecularly imprinted polymer synthesized by the epitope approach. Journal of Chromatography A 2000, 889 (1-2), 111-118.

12. Rachkov, A.; Minoura, N., Towards molecularly imprinted polymers selective to peptides and proteins. The epitope approach. Biochimica et Biophysica Acta (BBA)-Protein Structure and Molecular Enzymology 2001, 1544 (1-2), 255-266.

13. Cheong, W. J.; Yang, S. H.; Ali, F., Molecular imprinted polymers for separation science: a review of reviews. Journal of Separation Science 2013, 36 (3), 609-628.

14. Malitesta, C.; Mazzotta, E.; Picca, R. A.; Poma, A.; Chianella, I.; Piletsky, S. A., MIP sensors-the electrochemical approach. Analytical and Bioanalytical Chemistry 2012, 402 (5), 1827-1846. 
15. Wagner, S.; Bell, J.; Biyikal, M.; Gawlitza, K.; Rurack, K., Integrating fluorescent molecularly imprinted polymer (MIP) sensor particles with a modular microfluidic platform for nanomolar small-molecule detection directly in aqueous samples. Biosensors and Bioelectronics 2018, 99, 244-250.

16. Hilt, J. Z.; Byrne, M. E., Configurational biomimesis in drug delivery: molecular imprinting of biologically significant molecules. Advanced Drug Delivery Reviews 2004, 56 (11), 1599-1620.

17. Saridakis, E.; Khurshid, S.; Govada, L.; Phan, Q.; Hawkins, D.; Crichlow, G. V.; Lolis, E.; Reddy, S. M.; Chayen, N. E., Protein crystallization facilitated by molecularly imprinted polymers. Proceedings of the National Academy of Sciences 2011, 108 (27), 11081-11086.

18. Li, S.; Ge, Y.; Tiwari, A.; Wang, S.; Turner, A. P. F.; Piletsky, S. A., 'On/off'-switchable catalysis by a smart enzyme-like imprinted polymer. Journal of Catalysis 2011, 278 (2), 173-180.

19. Mahony, J. O.; Nolan, K.; Smyth, M. R.; Mizaikoff, B., Molecularly imprinted polymers-potential and challenges in analytical chemistry. Analytica Chimica Acta 2005, 534 (1), 31-39.

20. Boysen, R. I., Advances in the development of molecularly imprinted polymers for the separation and analysis of proteins with liquid chromatography. Journal of Separation Science 2019, 42 (1), 51-71.

21. Brocchieri, L.; Karlin, S., Protein length in eukaryotic and prokaryotic proteomes. Nucleic Acids Research 2005, 33 (10), 3390-3400.

22. Li, Q.; Shen, F.; Zhang, X.; Hu, Y.; Zhang, Q.; Xu, L.; Ren, X., One-pot synthesis of phenylphosphonic acid imprinted polymers for tyrosine phosphopeptides recognition in aqueous phase. Analytica Chimica Acta 2013, 795, 82-87.

23. Qin, Y.-P.; Li, D.-Y.; He, X.-W.; Li, W.-Y.; Zhang, Y.-K., Preparation of high-efficiency cytochrome cimprinted polymer on the surface of magnetic carbon nanotubes by epitope approach via metal chelation and six-membered ring. ACS Applied Materials \& Interfaces 2016, 8 (16), 10155-10163.

24. Yang, F.; Deng, D.; Dong, X.; Lin, S., Preparation of an epitope-imprinted polymer with antibodylike selectivity for beta2-microglobulin and application in serum sample analysis with a facile method of on-line solid-phase extraction coupling with high performance liquid chromatography. Journal of Chromatography A 2017, 1494, 18-26.

25. Jiang, W.; Liu, L.; Chen, Y., Simultaneous detection of human C-terminal p53 isoforms by single template molecularly imprinted polymers (MIPs) coupled with liquid chromatography-tandem mass spectrometry (LC-MS/MS)-based targeted proteomics. Analytical chemistry 2018, 90 (5), 3058-3066. 
26. Zhou, J.; Gan, N.; Li, T.; Hu, F.; Li, X.; Wang, L.; Zheng, L., A cost-effective sandwich electrochemiluminescence immunosensor for ultrasensitive detection of HIV-1 antibody using magnetic molecularly imprinted polymers as capture probes. Biosensors and Bioelectronics 2014, $54,199-206$.

27. Cenci, L.; Guella, G.; Andreetto, E.; Ambrosi, E.; Anesi, A.; Bossi, A. M., Guided folding takes a start from the molecular imprinting of structured epitopes. Nanoscale 2016, 8 (34), 15665-15670.

28. Zhang, Y.; Fonslow, B. R.; Shan, B.; Baek, M.-C.; Yates lii, J. R., Protein analysis by shotgun/bottom-up proteomics. Chemical Reviews 2013, 113 (4), 2343-2394.

29. Anderson, N. L.; Jackson, A.; Smith, D.; Hardie, D.; Borchers, C.; Pearson, T. W., SISCAPA peptide enrichment on magnetic beads using an in-line bead trap device. Molecular \& Cellular Proteomics 2009, 8 (5), 995-1005.

30. Fonslow, B. R.; Stein, B. D.; Webb, K. J.; Xu, T.; Choi, J.; Park, S. K.; Yates, J. R., Digestion and depletion of abundant proteins improves proteomic coverage. Nature Methods 2013, 10 (1), 54 56.

31. Biffis, A.; Dvorakova, G.; Falcimaigne-Cordin, A., Physical forms of MIPs. In Molecular Imprinting, Springer: 2010; pp 29-82.

32. Guiochon, G., Monolithic columns in high-performance liquid chromatography. Journal of Chromatography A 2007, 1168 (1-2), 101-168.

33. Wang, Q. C.; Svec, F.; Frechet, J. M. J., Macroporous polymeric stationary-phase rod as continuous separation medium for reversed-phase chromatography. Analytical Chemistry 1993, 65 (17), 2243-2248.

34. Masini, J. C.; Svec, F., Porous monoliths for on-line sample preparation: a review. Analytica Chimica Acta 2017, 964, 24-44.

35. Tan, J.; Jiang, Z.-T.; Li, R.; Yan, X.-P., Molecularly-imprinted monoliths for sample treatment and separation. Trends in Analytical Chemistry 2012, 39, 207-217.

36. Ye, L.; Mosbach, K., Molecularly imprinted microspheres as antibody binding mimics. Reactive and Functional Polymers 2001, 48 (1-3), 149-157.

37. Zhang, Z.; Chen, L.; Yang, F.; Li, J., Uniform core-shell molecularly imprinted polymers: a correlation study between shell thickness and binding capacity. RSC Advances 2014, 4 (60), 3150731514

38. Wan, L.; Chen, Z.; Huang, C.; Shen, X., Core-shell molecularly imprinted particles. Trends in Analytical Chemistry 2017, 95, 110-121. 
39. Gao, B.; Wang, J.; An, F.; Liu, Q., Molecular imprinted material prepared by novel surface imprinting technique for selective adsorption of pirimicarb. Polymer 2008, 49 (5), 1230-1238.

40. Tighe, P. J.; Ryder, R. R.; Todd, I.; Fairclough, L. C., ELISA in the multiplex era: potentials and pitfalls. PROTEOMICS-Clinical Applications 2015, 9 (3-4), 406-422.

41. Huang, S.; Xu, J.; Zheng, J.; Zhu, F.; Xie, L.; Ouyang, G., Synthesis and application of magnetic molecularly imprinted polymers in sample preparation. Analytical and Bioanalytical Chemistry 2018, 410 (17), 3991-4014.

42. Chen, L.; Li, B., Application of magnetic molecularly imprinted polymers in analytical chemistry. Analytical Methods 2012, 4 (9), 2613-2621.

43. Li, S.; Ge, Y.; Piletsky, S. A.; Lunec, J., Molecularly imprinted sensors: overview and applications. Elsevier: 2012.

44. Whitcombe, M. J.; Chianella, I.; Larcombe, L.; Piletsky, S. A.; Noble, J.; Porter, R.; Horgan, A., The rational development of molecularly imprinted polymer-based sensors for protein detection. Chemical Society Reviews 2011, 40 (3), 1547-1571.

45. Yoshikawa, M.; Tharpa, K.; Dima, S. O., Molecularly imprinted membranes: Past, present, and future. Chemical Reviews 2016, 116 (19), 11500-11528.

46. Çakir, P.; Cutivet, A.; Resmini, M.; Bui, B. T. S.; Haupt, K., Protein-size molecularly imprinted polymer nanogels as synthetic antibodies, by localized polymerization with multi-initiators. Advanced Materials 2013, 25 (7), 1048-1051.

47. Hortin, G. L.; Carr, S. A.; Anderson, N. L., Introduction: advances in protein analysis for the clinical laboratory. Clinical Chemistry 2010, 56 (2), 149.

48. Kosti, I.; Jain, N.; Aran, D.; Butte, A. J.; Sirota, M., Cross-tissue analysis of gene and protein expression in normal and cancer tissues. Scientific Reports 2016, 6 (1), 1-16.

49. Cavalieri, F., Drugs that target gene expression: an overview. Critical Reviews ${ }^{T M}$ in Eukaryotic Gene Expression 1996, 6 (1), 75-85.

50. Vogel, C.; Silva, G. M.; Marcotte, E. M., Protein expression regulation under oxidative stress. Molecular \& Cellular Proteomics 2011, 10 (12), M111-009217.

51. $\mathrm{Wu}, \mathrm{A}$. H. B., A selected history and future of immunoassay development and applications in clinical chemistry. Clinica Chimica Acta 2006, 369 (2), 119-124.

52. Fenn, J. B.; Mann, M.; Meng, C. K.; Wong, S. F.; Whitehouse, C. M., Electrospray ionization for mass spectrometry of large biomolecules. Science 1989, 246 (4926), 64-71. 
53. Carr, S. A.; Anderson, L., Protein quantitation through targeted mass spectrometry: the way out of biomarker purgatory? Clinical Chemistry 2008, 54 (11), 1749-52.

54. Di Meo, A.; Diamandis, E. P.; Rodriguez, H.; Hoofnagle, A. N.; loannidis, J.; Lopez, M., What is wrong with clinical proteomics? Clinical Chemistry 2014, 60 (10), 1258-1266.

55. Shibayama, T.; Ueoka, H.; Nishii, K.; Kiura, K.; Tabata, M.; Miyatake, K.; Kitajima, T.; Harada, M., Complementary roles of pro-gastrin-releasing peptide (ProGRP) and neuron specific enolase (NSE) in diagnosis and prognosis of small-cell lung cancer (SCLC). Lung Cancer 2001, 32 (1), 61-69.

56. Rifai, N.; Gillette, M. A.; Carr, S. A., Protein biomarker discovery and validation: the long and uncertain path to clinical utility. Nature Biotechnology 2006, 24 (8), 971-983.

57. Vashist, S. K.; Luong, J. H. T., Handbook of immunoassay technologies: approaches, performances, and applications. Academic Press: 2018.

58. Yalow, R. S., Radioimmunoassay. Annual Review of Biophysics and Bioengineering 1980, 9 (1), 327345.

59. Porsch-Özcürümez, M.; Kischel, N.; Priebe, H.; Splettstösser, W.; Finke, E.-J.; Grunow, R., Comparison of enzyme-linked immunosorbent assay, Western blotting, microagglutination, indirect immunofluorescence assay, and flow cytometry for serological diagnosis of tularemia. Clinical and Diagnostic Laboratory Immunology 2004, 11 (6), 1008-1015.

60. Engvall, E.; Perlmann, P., ENZYME-LINKED IMMUNOSORBENT ASSAY (ELISA) QUANTITATIVE ASSAY OF IMMUNOGLOBULIN-G. Immunochemistry 1971, 8 (9), 871-874.

61. Engvall, E.; Perlmann, P., Enzyme-linked immunosorbent assay, ELISA: III. Quantitation of specific antibodies by enzyme-labeled anti-immunoglobulin in antigen-coated tubes. The Journal of Immunology 1972, 109 (1), 129-135.

62. Aydin, S., A short history, principles, and types of ELISA, and our laboratory experience with peptide/protein analyses using ELISA. Peptides 2015, 72, 4-15.

63. Lequin, R. M., Enzyme Immunoassay (EIA)/Enzyme-Linked Immunosorbent Assay (ELISA). Clinical Chemistry 2005, 51 (12), 2415-2418.

64. Gan, S. D.; Patel, K. R., Enzyme immunoassay and enzyme-linked immunosorbent assay. Journal of Investigative Dermatology 2013, 133 (9), e12.

65. Schots, A.; Van der Leede, B. J.; De Jongh, E.; Egberts, E., A method for the determination of antibody affinity using a direct ELISA. Journal of Immunological Methods 1988, 109 (2), 225-233.

66. Berg, J. M.; Stryer, L.; Tymoczko, J. L., Stryer Biochemie. Springer-Verlag: 2015. 
67. Dobrovolskaia, E.; Gam, A.; Slater, J. E., Competition enzyme-linked immunosorbant assay (ELISA) can be a sensitive method for the specific detection of small quantities of allergen in a complex mixture. Clinical \& Experimental Allergy 2006, 36 (4), 525-530.

68. Anderson, N. L., The clinical plasma proteome: a survey of clinical assays for proteins in plasma and serum. Clinical Chemistry 2010, 56 (2), 177-185.

69. Immunoassay Market Size, Share \& Trends Analysis Report By Product (Reagents \& Kits, Software \& Services), By Technology (RIA, EIA, Rapid Test), By Application, By End Use, And Segment Forecasts, 2019 - 2025; GVR-3-68038-066-8; Grand View Research, 2019.

70. Manole, E.; Bastian, A. E.; Popescu, I. D.; Constantin, C.; Mihai, S.; Gaina, G. F.; Codrici, E.; Neagu, M. T., Immunoassay techniques highlighting biomarkers in immunogenetic diseases. In Immunogenetics, IntechOpen: 2018.

71. Ellington, A. A.; Kullo, I. J.; Bailey, K. R.; Klee, G. G., Antibody-based protein multiplex platforms: technical and operational challenges. Clinical Chemistry 2010, 56 (2), 186-193.

72. Ling, M. M.; Ricks, C.; Lea, P., Multiplexing molecular diagnostics and immunoassays using emerging microarray technologies. Expert Review of Molecular Diagnostics 2007, 7 (1), 87-98.

73. Juncker, D.; Bergeron, S.; Laforte, V.; Li, H., Cross-reactivity in antibody microarrays and multiplexed sandwich assays: shedding light on the dark side of multiplexing. Current Opinion in Chemical Biology 2014, 18, 29-37.

74. Lee, J. R.; Lee, J.; Kim, S. K.; Kim, K. P.; Park, H. S.; Yeo, W. S., Mass spectrometry signal amplification method for attomolar detection of antigens using small-molecule-tagged gold microparticles. Angewandte Chemie International Edition 2008, 47 (49), 9518-21.

75. Aebersold, R.; Mann, M., Mass spectrometry-based proteomics. Nature 2003, 422 (6928), 198207.

76. Karas, M.; Hillenkamp, F., Laser desorption ionization of proteins with molecular masses exceeding 10,000 daltons. Analytical chemistry 1988, 60 (20), 2299-2301.

77. Loo, J. A., Studying noncovalent protein complexes by electrospray ionization mass spectrometry. Mass Spectrometry Reviews 1997, 16 (1), 1-23.

78. Ho, C. S.; Lam, C. W. K.; Chan, M. H. M.; Cheung, R. C. K.; Law, L. K.; Lit, L. C. W.; Ng, K. F.; Suen, M. W. M.; Tai, H. L., Electrospray ionisation mass spectrometry: principles and clinical applications. The Clinical Biochemist Reviews 2003, 24 (1), 3-12.

79. Bruins, A. P., Mechanistic aspects of electrospray ionization. Journal of Chromatography A 1998, 794 (1-2), 345-357. 
80. Duncan, M. W.; Roder, H.; Hunsucker, S. W., Quantitative matrix-assisted laser desorption/ionization mass spectrometry. Briefings in Functional Genomics and Proteomics 2008, 7 (5), 355-370.

81. El-Aneed, A.; Cohen, A.; Banoub, J., Mass spectrometry, review of the basics: electrospray, MALDI, and commonly used mass analyzers. Applied Spectroscopy Reviews 2009, 44 (3), 210-230.

82. Anderson, L.; Hunter, C. L., Quantitative mass spectrometric multiple reaction monitoring assays for major plasma proteins. Molecular \& Cellular Proteomics 2006, 5 (4), 573-588.

83. Yost, R.; Enke, C., Selected ion fragmentation with a tandem quadrupole mass spectrometer. Journal of the American Chemical Society 1978, 100 (7), 2274-2275.

84. McLafferty, F. W.; Bente, P. F.; Kornfeld, R.; Tsai, S.-C.; Howe, I., Metastable ion characteristics. XXII. Collisional activation spectra of organic ions. Journal of the American Chemical Society 1973, 95 (7), 2120-2129.

85. Hoofnagle, A. N.; Wener, M. H., The fundamental flaws of immunoassays and potential solutions using tandem mass spectrometry. Journal of Immunological Methods 2009, 347 (1-2), 3-11.

86. Wang, H.; Shi, T.; Qian, W.-J.; Liu, T.; Kagan, J.; Srivastava, S.; Smith, R. D.; Rodland, K. D.; Camp, D. G., The clinical impact of recent advances in LC-MS for cancer biomarker discovery and verification. Expert Review of Proteomics 2016, 13 (1), 99-114.

87. Bashyal, A.; Sanders, J. D.; Holden, D. D.; Brodbelt, J. S., Top-Down Analysis of Proteins in Low Charge States. Journal of the American Society for Mass Spectrometry 2019, 30 (4), 704-717.

88. Capriotti, A. L.; Cavaliere, C.; Foglia, P.; Samperi, R.; Laganà, A., Intact protein separation by chromatographic and/or electrophoretic techniques for top-down proteomics. Journal of Chromatography A 2011, 1218 (49), 8760-76.

89. Kelleher, N. L., Peer Reviewed: Top-Down Proteomics. Analytical Chemistry 2004, 76 (11), 196 A$203 \mathrm{~A}$.

90. Compton, P. D.; Zamdborg, L.; Thomas, P. M.; Kelleher, N. L., On the scalability and requirements of whole protein mass spectrometry. Analytical Chemistry 2011, 83 (17), 6868-74.

91. Catherman, A. D.; Skinner, O. S.; Kelleher, N. L., Top Down proteomics: facts and perspectives. Biochemical and Biophysical Research Communications 2014, 445 (4), 683-693.

92. Taverna, S. D.; Ueberheide, B. M.; Liu, Y.; Tackett, A. J.; Diaz, R. L.; Shabanowitz, J.; Chait, B. T.; Hunt, D. F.; Allis, C. D., Long-distance combinatorial linkage between methylation and acetylation on histone H3 N termini. Proceedings of the National Academy of Sciences 2007, 104 (7), 20862091. 
93. Sidoli, S.; Garcia, B. A., Middle-down proteomics: a still unexploited resource for chromatin biology. Expert Review of Proteomics 2017, 14 (7), 617-626.

94. Tvardovskiy, A.; Wrzesinski, K.; Sidoli, S.; Fey, S. J.; Rogowska-Wrzesinska, A.; Jensen, O. N., Topdown and middle-down protein analysis reveals that intact and clipped human histones differ in post-translational modification patterns. Molecular \& Cellular Proteomics 2015, 14 (12), 31423153.

95. Gillet, L. C.; Leitner, A.; Aebersold, R., Mass spectrometry applied to bottom-up proteomics: entering the high-throughput era for hypothesis testing. Annual Review of Analytical Chemistry 2016, 9, 449-472.

96. Getz, E. B.; Xiao, M.; Chakrabarty, T.; Cooke, R.; Selvin, P. R., A comparison between the sulfhydryl reductants tris (2-carboxyethyl) phosphine and dithiothreitol for use in protein biochemistry. Analytical biochemistry 1999, 273 (1), 73-80.

97. Konigsberg, W., [13] Reduction of disulfide bonds in proteins with dithiothreitol. In Methods in Enzymology, Academic Press: 1972; Vol. 25, pp 185-188.

98. Singh, R.; Lamoureux, G. V.; Lees, W. J.; Whitesides, G. M., [14] Reagents for rapid reduction of disulfide bonds. In Methods in Enzymology, Academic Press: 1995; Vol. 251, pp 167-173.

99. Herbert, B.; Galvani, M.; Hamdan, M.; Olivieri, E.; MacCarthy, J.; Pedersen, S.; Righetti, P. G., Reduction and alkylation of proteins in preparation of two-dimensional map analysis: Why, when, and how? Electrophoresis 2001, 22 (10), 2046-2057.

100. Giansanti, P.; Tsiatsiani, L.; Low, T. Y.; Heck, A. J. R., Six alternative proteases for mass spectrometry-based proteomics beyond trypsin. Nature Protocols 2016, 11 (5), 993-1006.

101. Inagami, T.; Murachi, T., The mechanism of the specificity of trypsin catalysis. Journal of Biological Chemistry 1964, 239 (8), 1395-1401.

102. Finehout, E. J.; Cantor, J. R.; Lee, K. H., Kinetic characterization of sequencing grade modified trypsin. Proteomics 2005, 5 (9), 2319-2321.

103. Dey, K. K.; Wang, H.; Niu, M.; Bai, B.; Wang, X.; Li, Y.; Cho, J.-H.; Tan, H.; Mishra, A.; High, A. A.; Chen, P.-C.; Wu, Z.; Beach, T. G.; Peng, J., Deep undepleted human serum proteome profiling toward biomarker discovery for Alzheimer's disease. Clinical Proteomics 2019, 16 (1), 16.

104. Holm, A.; Wu, W.; Lund-Johansen, F., Antibody array analysis of labelled proteomes: how should we control specificity? New Biotechnology 2012, 29 (5), 578-585. 
105. Fonslow, B. R.; Stein, B. D.; Webb, K. J.; Xu, T.; Choi, J.; Park, S. K.; Yates, J. R., Digestion and depletion of abundant proteins improves proteomic coverage. Nature Methods 2013, 10 (1), 54 56.

106. Nelson, R. E.; Grebe, S. K.; O’Kane, D. J.; Singh, R. J., Liquid Chromatography-Tandem Mass Spectrometry Assay for Simultaneous Measurement of Estradiol and Estrone in Human Plasma. Clinical Chemistry 2004, 50 (2), 373-384.

107. Torsetnes, S. B.; Broughton, M. N.; Paus, E.; Halvorsen, T. G.; Reubsaet, L., Determining ProGRP and isoforms in lung and thyroid cancer patient samples: comparing an MS method with a routine clinical immunoassay. Analytical and Bioanalytical Chemistry Research 2014, 406 (11), 2733-2738.

108. Portelius, E.; Westman-Brinkmalm, A.; Zetterberg, H.; Blennow, K., Determination of $\beta$-amyloid peptide signatures in cerebrospinal fluid using immunoprecipitation-mass spectrometry. Journal of Proteome Research 2006, 5 (4), 1010-1016.

109. Torsetnes, S. B.; Løvbak, S. G.; Claus, C.; Lund, H.; Nordlund, M. S.; Paus, E.; Halvorsen, T. G.; Reubsaet, L., Immunocapture and LC-MS/MS for selective quantification and differentiation of the isozymes of the biomarker neuron-specific enolase in serum. Journal of Chromatography $B$ 2013, 929, 125-132.

110. Berna, M.; Ott, L.; Engle, S.; Watson, D.; Solter, P.; Ackermann, B., Quantification of NTproBNP in rat serum using immunoprecipitation and LC/MS/MS: a biomarker of drug-induced cardiac hypertrophy. Analytical Chemistry 2008, 80 (3), 561-566.

111. Kumar, V.; Barnidge, D. R.; Chen, L.-S.; Twentyman, J. M.; Cradic, K. W.; Grebe, S. K.; Singh, R. J., Quantification of serum 1-84 parathyroid hormone in patients with hyperparathyroidism by immunocapture in situ digestion liquid chromatography-tandem mass spectrometry. Clinical Chemistry 2010, 56 (2), 306-313.

112. Schoenherr, R. M.; Zhao, L.; Ivey, R. G.; Voytovich, U. J.; Kennedy, J.; Yan, P.; Lin, C.; Whiteaker, J. R.; Paulovich, A., Commercially available antibodies can be applied in quantitative multiplexed peptide immunoaffinity enrichment targeted mass spectrometry assays. Proteomics 2016, 16 (15-16), 2141-2145.

113. Nicol, G. R.; Han, M.; Kim, J.; Birse, C. E.; Brand, E.; Nguyen, A.; Mesri, M.; FitzHugh, W.; Kaminker, P.; Moore, P. A., Use of an immunoaffinity-mass spectrometry-based approach for the quantification of protein biomarkers from serum samples of lung cancer patients. Molecular \& Cellular Proteomics 2008, 7 (10), 1974-1982. 
114. Lin, D.; Alborn, W. E.; Slebos, R. J.; Liebler, D. C., Comparison of protein immunoprecipitationmultiple reaction monitoring with ELISA for assay of biomarker candidates in plasma. Journal of Proteome Research 2013, 12 (12), 5996-6003.

115. Torsetnes, S. B.; Levernæs, M. S.; Broughton, M. N.; Paus, E.; Halvorsen, T. G.; Reubsaet, L., Multiplexing determination of small cell lung cancer biomarkers and their isovariants in serum by immunocapture LC-MS/MS. Analytical Chemistry 2014, 86 (14), 6983-6992.

116. Burow, M.; Minoura, N., Molecular imprinting: synthesis of polymer particles with antibody-like binding characteristics for glucose oxidase. Biochemical and Biophysical Research Communications 1996, 227 (2), 419-422.

117. Rachkov, A.; Hu, M.; Bulgarevich, E.; Matsumoto, T.; Minoura, N., Molecularly imprinted polymers prepared in aqueous solution selective for [Sar1, Ala8] angiotensin II. Analytica Chimica Acta 2004, 504 (1), 191-197.

118. Hart, B. R.; Shea, K. J., Molecular imprinting for the recognition of $\mathrm{N}$-terminal histidine peptides in aqueous solution. Macromolecules 2002, 35 (16), 6192-6201.

119. Bossi, A. M.; Sharma, P. S.; Montana, L.; Zoccatelli, G.; Laub, O.; Levi, R., Fingerprint-imprinted polymer: rational selection of peptide epitope templates for the determination of proteins by molecularly imprinted polymers. Analytical Chemistry 2012, 84 (9), 4036-4041.

120. Rossetti, C.; Świtnicka-Plak, M. A.; Halvorsen, T. G.; Cormack, P. A.; Sellergren, B.; Reubsaet, L., Automated Protein Biomarker Analysis: on-line extraction of clinical samples by Molecularly Imprinted Polymers. Scientific Reports 2017, 7, 44298.

121. Shinde, S.; Bunschoten, A.; Kruijtzer, J. A.; Liskamp, R. M.; Sellergren, B., Imprinted polymers displaying high affinity for sulfated protein fragments. Angewandte Chemie International Edition 2012, 51 (33), 8326-8329.

122. Basan, H.; Dinc, M.; Mizaikoff, B., Inhibitor-assisted synthesis of molecularly imprinted microbeads for protein recognition. Analytical Methods 2018, 10(9), 997-1005.

123. Tang, A.-n.; Duan, L.; Liu, M.; Dong, X., An epitope imprinted polymer with affinity for kininogen fragments prepared by metal coordination interaction for cancer biomarker analysis. Journal of Materials Chemistry B 2016, 4 (46), 7464-7471.

124. Gao, X.; Hu, X.; Guan, P.; Du, C.; Ding, S.; Zhang, X.; Li, B.; Wei, X.; Song, R., Synthesis of coreshell imprinting polymers with uniform thin imprinting layer via iniferter-induced radical polymerization for the selective recognition of thymopentin in aqueous solution. RSC Advances 2016, 6 (111), 110019-110031. 
125. Lin, Z.; Sun, L.; Liu, W.; Xia, Z.; Yang, H.; Chen, G., Synthesis of boronic acid-functionalized molecularly imprinted silica nanoparticles for glycoprotein recognition and enrichment. Journal of Materials Chemistry B 2014, 2 (6), 637-643.

126. Hirayama, K.; Sakai, Y.; Kameoka, K., Synthesis of polymer particles with specific lysozyme recognition sites by a molecular imprinting technique. Journal of Applied Polymer Science 2001, 81 (14), 3378-3387.

127. Lulka, M. F.; Iqbal, S. S.; Chambers, J. P.; Valdes, E. R.; Thompson, R. G.; Goode, M. T.; Valdes, J. J., Molecular imprinting of Ricin and its A and B chains to organic silanes: fluorescence detection. Materials Science and Engineering: C 2000, 11 (2), 101-105.

128. Bertolla, M.; Cenci, L.; Anesi, A.; Ambrosi, E.; Tagliaro, F.; Vanzetti, L.; Guella, G.; Bossi, A. M., Solvent-responsive molecularly imprinted nanogels for targeted protein analysis in MALDI-TOF mass spectrometry. ACS Applied Materials \& Interfaces 2017, 9 (8), 6908-6915.

129. Liu, D.; Yang, Q.; Jin, S.; Song, Y.; Gao, J.; Wang, Y.; Mi, H., Core-shell molecularly imprinted polymer nanoparticles with assistant recognition polymer chains for effective recognition and enrichment of natural low-abundance protein. Acta Biomaterialia 2014, 10 (2), 769-775.

130. Hoshino, Y.; Koide, H.; Urakami, T.; Kanazawa, H.; Kodama, T.; Oku, N.; Shea, K. J., Recognition, Neutralization, and Clearance of Target Peptides in the Bloodstream of Living Mice by Molecularly Imprinted Polymer Nanoparticles: A Plastic Antibody. Journal of the American Chemical Society 2010, 132 (19), 6644-6645.

131. Hoshino, Y.; Kodama, T.; Okahata, Y.; Shea, K. J., Peptide imprinted polymer nanoparticles: a plastic antibody. Journal of the American Chemical Society 2008, 130 (46), 15242-15243.

132. Zhang, Z.; Yang, X.; Chen, X.; Zhang, M.; Luo, L.; Peng, M.; Yao, S., Novel magnetic bovine serum albumin imprinted polymers with a matrix of carbon nanotubes, and their application to protein separation. Analytical and Bioanalytical Chemistry 2011, 401 (9), 2855.

133. Tan, C. J.; Chua, H. G.; Ker, K. H.; Tong, Y. W., Preparation of bovine serum albumin surfaceimprinted submicrometer particles with magnetic susceptibility through core- shell miniemulsion polymerization. Analytical Chemistry 2008, 80 (3), 683-692.

134. Tan, L.; Yu, Z.; Zhou, X.; Xing, D.; Luo, X.; Peng, R.; Tang, Y., Antibody-free ultra-high performance liquid chromatography/tandem mass spectrometry measurement of angiotensin I and II using magnetic epitope-imprinted polymers. Journal of Chromatography A 2015, 1411, 6976. 
135. Liu, Y.; Wang, Y.; Dai, Q.; Zhou, Y., Magnetic deep eutectic solvents molecularly imprinted polymers for the selective recognition and separation of protein. Analytica Chimica Acta 2016, 936, 168-178.

136. Kan, X.; Zhao, Q.; Shao, D.; Geng, Z.; Wang, Z.; Zhu, J.-J., Preparation and Recognition Properties of Bovine Hemoglobin Magnetic Molecularly Imprinted Polymers. The Journal of Physical Chemistry B 2010, 114 (11), 3999-4004.

137. Zhou, W.-H.; Lu, C.-H.; Guo, X.-C.; Chen, F.-R.; Yang, H.-H.; Wang, X.-R., Mussel-inspired molecularly imprinted polymer coating superparamagnetic nanoparticles for protein recognition. Journal of Materials Chemistry 2010, 20 (5), 880-883.

138. Lee, M.-H.; Thomas, J. L.; Ho, M.-H.; Yuan, C.; Lin, H.-Y., Synthesis of Magnetic Molecularly Imprinted Poly(ethylene-co-vinyl alcohol) Nanoparticles and Their Uses in the Extraction and Sensing of Target Molecules in Urine. ACS Applied Materials \& Interfaces 2010, 2 (6), 1729-1736.

139. Jing, T.; Xia, H.; Guan, Q.; Lu, W.; Dai, Q.; Niu, J.; Lim, J.-M.; Hao, Q.; Lee, Y.-I.; Zhou, Y.; Mei, S., Rapid and selective determination of urinary lysozyme based on magnetic molecularly imprinted polymers extraction followed by chemiluminescence detection. Analytica Chimica Acta 2011, 692 (1), 73-79.

140. Xu, K.; Wang, Y.; Wei, X.; Chen, J.; Xu, P.; Zhou, Y., Preparation of magnetic molecularly imprinted polymers based on a deep eutectic solvent as the functional monomer for specific recognition of lysozyme. Microchimica Acta 2018, 185 (2), 146.

141. Gai, Q.-Q.; Qu, F.; Liu, Z.-J.; Dai, R.-J.; Zhang, Y.-K., Superparamagnetic lysozyme surfaceimprinted polymer prepared by atom transfer radical polymerization and its application for protein separation. Journal of Chromatography A 2010, 1217 (31), 5035-5042.

142. Tan, C. J.; Tong, Y. W., The effect of protein structural conformation on nanoparticle molecular imprinting of ribonuclease A using miniemulsion polymerization. Langmuir 2007, 23 (5), 2722 2730.

143. Liu, Y.; Gu, Y.; Li, M.; Wei, Y., Protein imprinting over magnetic nanospheres via a surface grafted polymer for specific capture of hemoglobin. New Journal of Chemistry 2014, 38 (12), 6064-6072.

144. Afsarimanesh, N.; Mukhopadhyay, S. C.; Kruger, M., Molecularly Imprinted Polymer-Based Electrochemical Biosensor for Bone Loss Detection. IEEE Transactions on Biomedical Engineering 2017, 65 (6). 
145. Dechtrirat, D.; Jetzschmann, K. J.; Stöcklein, W. F.; Scheller, F. W.; Gajovic-Eichelmann, N., Protein Rebinding to a Surface-Confined Imprint. Advanced Functional Materials 2012, 22 (24), 5231-5237.

146. Jetzschmann, K. J.; Yarman, A.; Rustam, L.; Kielb, P.; Urlacher, V. B.; Fischer, A.; Weidinger, I. M.; Wollenberger, U.; Scheller, F. W., Molecular LEGO by domain-imprinting of cytochrome P450 BM3. Colloids and Surfaces B: Biointerfaces 2018, 164, 240-246.

147. Johari-Ahar, M.; Karami, P.; Ghanei, M.; Afkhami, A.; Bagheri, H., Development of a molecularly imprinted polymer tailored on disposable screen-printed electrodes for dual detection of EGFR and VEGF using nano-liposomal amplification strategy. Biosensors and Bioelectronics 2018.

148. Stojanovic, Z.; Erdőssy, J.; Keltai, K.; Scheller, F. W.; Gyurcsányi, R. E., Electrosynthesized molecularly imprinted polyscopoletin nanofilms for human serum albumin detection. Analytica Chimica Acta 2017, 977, 1-9.

149. Lee, M.-H.; Thomas, J. L.; Liao, C.-L.; Jurcevic, S.; Crnogorac-Jurcevic, T.; Lin, H.-Y., Polymers imprinted with three REG1B peptides for electrochemical determination of Regenerating Protein 1B, a urinary biomarker for pancreatic ductal adenocarcinoma. Microchimica Acta 2017, 184 (6), $1773-1780$

150. Karimian, N.; Vagin, M.; Zavar, M. H. A.; Chamsaz, M.; Turner, A. P. F.; Tiwari, A., An ultrasensitive molecularly-imprinted human cardiac troponin sensor. Biosensors and Bioelectronics 2013, 50, 492-498.

151. Winther, B.; Moi, P.; Nordlund, M. S.; Lunder, N.; Paus, E.; Reubsaet, J. L. E., Absolute ProGRP quantification in a clinical relevant concentration range using LC-MS/MS and a comprehensive internal standard. Journal of Chromatography B 2009, 877 (13), 1359-1365.

152. Molina, R.; Filella, X.; Auge, J. M., ProGRP: a new biomarker for small cell lung cancer. Clinical Biochemistry 2004, 37 (7), 505-511.

153. Wojcik, E.; Kulpa, J. K., Pro-gastrin-releasing peptide (ProGRP) as a biomarker in small-cell lung cancer diagnosis, monitoring and evaluation of treatment response. Lung Cancer: Targets and Therapy 2017, 8, 231.

154. Pancholi, V., Multifunctional $\alpha$-enolase: its role in diseases. Cellular and Molecular Life Sciences 2001, 58 (7), 902-920.

155. Wang, X.; Wang, Y.; Ye, X.; Wu, T.; Deng, H.; Wu, P.; Li, C., Sensing platform for neuron specific enolase based on molecularly imprinted polymerized ionic liquids in between gold nanoarrays. Biosensors and Bioelectronics 2018, 99, 34-39. 
156. Esscher, T.; Steinholtz, L.; Bergh, J.; Nöu, E.; Nilsson, K.; Påhlman, S., Neurone specific enolase: a useful diagnostic serum marker for small cell carcinoma of the lung. Thorax 1985, 40 (2), 85-90.

157. Lin, Z.; Cheng, W.; Li, Y.; Liu, Z.; Chen, X.; Huang, C., A novel superparamagnetic surface molecularly imprinted nanoparticle adopting dummy template: An efficient solid-phase extraction adsorbent for bisphenol A. Analytica Chimica Acta 2012, 720, 71-76.

158. Rossetti, C.; Abdel Qader, A.; Halvorsen, T. G.; Sellergren, B.; Reubsaet, L., Antibody-free biomarker determination: Exploring molecularly imprinted polymers for pro-gastrin releasing peptide. Analytical Chemistry 2014, 86 (24), 12291-12298.

159. Lime, F.; Irgum, K., Preparation of divinylbenzene and divinylbenzene-co-glycidyl methacrylate particles by photoinitiated precipitation polymerization in different solvent mixtures. Macromolecules 2009, 42 (13), 4436-4442.

160. Vasapollo, G.; Sole, R. D.; Mergola, L.; Lazzoi, M. R.; Scardino, A.; Scorrano, S.; Mele, G., Molecularly imprinted polymers: present and future prospective. International Journal of Molecular Sciences 2011, 12 (9), 5908-5945.

161. Rossetti, C.; Levernæs, M. C. S.; Reubsaet, L.; Halvorsen, T. G., Evaluation of affinity-based serum clean-up in mass spectrometric analysis: Plastic vs monoclonal antibodies. Journal of Chromatography A 2016, 1471, 19-26. 



\title{
Magnetic Synthetic Receptors for Selective Clean-Up in Protein Biomarker Quantification
}

\author{
Nicholas McKitterick, Frida Braathen, Magdalena A. Switnicka-Plak, Peter A. G. Cormack,* \\ Léon Reubsaet, and Trine Grønhaug Halvorsen*
}

Cite This: J. Proteome Res. 2020, 19, 3573-3582

Read Online

ACCESS | W Metrics \& More | 回国 Article Recommendations | S1 Supporting Information

ABSTRACT: Biomarker analysis by mass spectrometry (MS) can allow for the rapid quantification of low abundant biomarkers. However, the complexity of human serum is a limiting factor in MS-based bioanalysis; therefore, novel biomarker enrichment strategies are of interest, particularly if the enrichment strategies are of low cost and are easy to use. One such strategy involves the use of molecularly imprinted polymers (MIPs) as synthetic receptors for biomarker enrichment. In the present study, a magnetic solid-phase extraction ( $\mathrm{mSPE}$ ) platform, based on magnetic MIP (mMIP) sorbents, is disclosed, for use in the MS-based quantification of proteins by the bottom-up approach. Progastrin releasing peptide (ProGRP), a low abundant and clinically

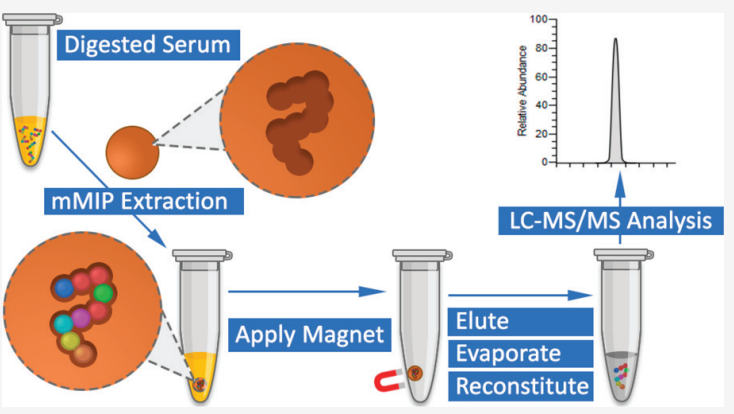
sensitive biomarker for small cell lung cancer (SCLC), was used to exemplify the mSPE platform. Four different mMIPs were synthesized, and an mSPE method was developed and optimized for the extraction of low concentrations of tryptic peptides from human serum. The mSPE method enabled the selective extraction of the ProGRP signature peptide, the nonapeptide NLLGLIEAK, prior to quantification of the target via LC-MS/MS. Overall, the mSPE method demonstrated its potential as a low cost, rapid, and straightforward sample preparation method, with demonstrably strong binding, acceptable recoveries, and good compatibility with MS. mMIPs are a potential low-cost alternative to current clinical methods for biomarker analysis.

KEYWORDS: LC-MS/MS, low-abundant biomarkers, bottom-up protein analysis, magnetic capture, molecularly imprinted polymers

\section{INTRODUCTION}

The role of biomarkers in the diagnosis and management of disease is an increasingly critical aspect of clinical pathology. Therefore, it is of utmost importance that there is robust, accurate, and rapid quantitation of biomarkers, and especially for biomarkers of aggressive diseases (e.g., cancers). Many serum biomarkers can be used to diagnose malignancies without the need for invasive procedures such as biopsies of internal organs. ${ }^{1}$

Low abundant protein biomarkers present analytical challenges in MS-based proteomics, namely difficulty in selective enrichment and quantification due to interference from high abundant proteins and other serum components. ${ }^{2}$ MS analysis of proteins is typically achieved using one of two approaches: top-down and bottom-up proteomics. Top-down approaches involve the analysis of whole proteins by MS, whereas bottom-up analysis involves enzymatic digestion of proteins and analysis of proteolytic peptides. The use of bottom-up workflows in tandem with enrichment methods has gained interest in recent years. LC-MS/MS analysis of signature peptides has the potential to yield high accuracy and precision, with low limits of detection (LOD) compared to top-down analysis, metrics that are essential when quantifying low abundant biomarkers. ${ }^{3}$ To utilize fully the quantitative potential of bottom-up LC-MS/MS, selective enrichment of the target biomarker marker is critical. Typically, antibodybased selective enrichment has been used in sample cleanup owing to the high selectivity of antibodies for targets. However, producing highly selective antibodies is expensive, laborious, complex, and time-consuming. Therefore, alternative materials with molecularly selective binding properties are desirable, and MIPs are one such alternative in this regard.

MIPs are robust, synthetic polymers designed to have unique chemical and structural properties that allow selective recognition of a desired target. ${ }^{4}$ These properties have been exploited to allow MIPs to bind strongly and selectively to a variety of targets, from small molecules to large macromolecular targets such as proteins, and even to cells. ${ }^{5}$ Usually, MIPs bind to targets via noncovalent forces (including

Received: April 17, 2020

Published: July 2, 2020 
hydrogen bonding, electrostatic interactions, hydrophobic interactions, and van der Waals forces), although binding can also be through the formation of covalent bonds. ${ }^{6}$ Thus, MIPs can be considered to be antibody-binding mimics, and are sometimes even referred to as plastic antibodies. Compared to antibody production and use, MIPs are more cost-effective, reusable, and require less complex and time-consuming synthesis, and this has led to many applications for MIPs: they have been utilized successfully as solid-phase extraction (SPE) sorbents, ${ }^{7}$ electrochemical sensors, ${ }^{8}$ in drug delivery, ${ }^{9}$ for protein crystallization, ${ }^{10}$ and for catalysis. ${ }^{11}$ To date, MIPbased assays typically have higher detection limits compared to antibody-based immunoassay methods. However, magnetic MIPs (mMIPs) are a promising emerging format that has shown some promise for the extraction of peptides present at low levels in serum. ${ }^{12}$

mMIPs are MIPs with magnetic properties, and some of these materials have been developed to target and quantify peptides and proteins. ${ }^{13}$ Typically, mMIPs can be produced in one of two distinct ways: either by encapsulation of a preformed magnetic component during a template-directed synthesis ${ }^{14}$ or by magnetization of a MIP. ${ }^{15}$ mMIPs allow for the simplification of off-line SPE, with the use of a magnet allowing for the circumvention of several centrifugation steps to remove the sample matrix, ${ }^{16}$ greatly speeding up work-flow. mMIPs have been used for the extraction and top-down quantification of proteins such as bovine serum albumin (BSA),${ }^{17}$ lysozyme ${ }^{18}$ hemoglobin, ${ }^{13}$ and RNase A. ${ }^{19}$ However, the analysis of whole proteins (i.e., top-down proteomics) typically gives higher LODs because the MS analysis of whole proteins is less sensitive than (bottom-up) peptide analysis. MIPs targeting peptides have been shown to function well in complex matrices: An epitope imprinted MIP targeting the low abundant biomarker protein cardiac troponin I allowed enrichment of the target protein in a matrix designed to mimic human serum, ${ }^{20}$ an epitope imprinted MIP targeting the high abundant protein HTR was found to enrich the target protein qualitatively. ${ }^{21}$ Similarity, mMIPs have been shown to function in complex matrices: an mMIP targeting lysozyme demonstrated clear enrichment in egg white. ${ }^{22}$ While an mMIP targeting the peptide hormones angiotensin I and II demonstrated the value of mMIPs for the enrichment and quantification of peptides using LC-MS/MS, ${ }^{12}$ the use of mMIPs for target enrichment and cleanup in bottom-up proteomics has not yet been reported.

To demonstrate the ability of the mMIP platform to enable the determination of tryptic peptides, the small-cell lung cancer biomarker ProGRP is an appealing model because a fully validated LC-MS method has been developed for its tryptic peptides. ${ }^{23}$ Furthermore, ProGRP is a low abundant biomarker that is known to be clinically sensitive (most patients testing positive for ProGRP are in a diseased state) and selective (most patients testing negative are not in the diseased state). ${ }^{24}$ The signature peptide of ProGRP, NLLGLIEAK, is a very reproducibly produced tryptic peptide and has high MS sensitivity. Previously, nonmagnetic MIPs have been developed to extract NLLGLIEAK from serum using off-line MISPE ${ }^{25}$ and online MISPE (MISPE is molecularly imprinted SPE). ${ }^{26}$

The aim of the current work was to develop mMIPs targeting NLLGLIEAK and to explore the potential for the selective and rapid extraction of tryptic peptides in serum. Four mMIPs were designed and synthesized, and an mSPE method was developed and optimized using increasingly complex matrices to demonstrate the clinical viability of mMIPs for the extraction of NLLGLIEAK from human serum.

\section{MATERIALS AND METHODS}

\section{Chemicals and Reagents}

Acetonitrile LC-MS grade (MeCN, 99.9\%), methanol LC-MS grade $(\mathrm{MeOH}, 99.9 \%)$, acetic acid $(\mathrm{AcOH}, 100 \%)$, ethanol (EtOH, $\geq 99.5 \%$ ), and dimethyl sulfoxide (DMSO, $\geq 98 \%$ ) were purchased from Merck (Darmstadt, Germany). Ammonium bicarbonate (BioUltra, $\geq 99.5 \%$ ) was purchased from Fluka (Milwaukee, WI, USA). Formic acid (FA, MS grade, $\geq 98 \%$ ), divinylbenzene-80 (DVB-80, 80\%), methacrylic acid (MAA, purity $\geq 98.0 \%$ ), 1,2,2,6,6-pentamethylpiperidine (PMP, purity $>99 \%$ ), tetrabutylammonium hydroxide solution (TBA. OH, $1.0 \mathrm{M}$ in methanol, $\leq 50 \%)$, hydrochloric acid $(37 \%$ (w/w) in $\mathrm{H}_{2} \mathrm{O}$ ), Tween 20 , sodium hydroxide $(\mathrm{NaOH}$, purity $\geq 97 \%$ ), iron(III) chloride $\left(\mathrm{FeCl}_{3}\right.$, purity $\left.97 \%\right)$, iron(II) chloride $\left(\mathrm{FeCl}_{2}\right.$, purity $\left.98 \%\right)$ DL-dithiothreitol $(\geq 99.5 \%$, DTT), iodoacetic acid ( $\geq 98 \%$, IAA), and $28-30 \%$ ammonium hydroxide solution $\left(\mathrm{NH}_{4} \mathrm{OH}\right)$ were all purchased from SigmaAldrich (St. Louis, MO, USA). 2-Aminoethyl methacrylamide hydrochloride (EAMA $\cdot \mathrm{HCl}$, purity $\geq 98 \%$ ) was purchased from Polysciences Inc. (Niles, IL, USA). N-3,5-bis(Trifluoromethyl)-phenyl- $N^{\prime}-4$-vinylphenylurea (BTPV, purity $>95 \%$ ) is not commercially available and was kindly donated by Dortmund University. Z-NLLGLIEA[Nle] (purity 96.58\%) was purchased from LifeTein. 2,2'-Azo-bis-isobutyronitrile (AIBN, purity $98 \%$ ) was purchased from BDH Lab. Supplies (Dubai, UAE). Water was filtered through a Merck Millipore Milli-Q Integral 3 water dispenser (resistivity: $18.2 \mathrm{M} \Omega \mathrm{cm}^{-1}$ ).

Preparation of Reagents, Proteins, and Peptides. DVB-80 was purified by filtration through a short plug of neutral aluminum oxide prior to use. AIBN was recrystallized from acetone at low temperature.

Recombinant ProGRP was obtained from Radiumhospitalet, Oslo University Hospital, Oslo, Norway. ProGRP isoform 1 was cloned from human cDNA (Origene technologies) and expressed in Escherichia coli (Promega) via pGEX-6P-3 constructs (GE Healthcare) and purified as described previously. ${ }^{25}$ ProGRP concentrations were determined via UV absorbance $(280 \mathrm{~nm})$, diluted to the desired concentration with $50 \mathrm{mM}$ ammonium bicarbonate $(\mathrm{ABC})$ and stored at -20 ${ }^{\circ} \mathrm{C}$.

Synthetic NLLGLIEAK (>95\%) and the stable isotope labeled internal standard (IS) peptide NLLGLIEA$\left[\mathrm{K}_{-}{ }^{13} \mathrm{C}_{6}{ }^{15} \mathrm{~N}_{2}\right] \quad(>95 \%)$ were purchased from Innovagen (Lund, Sweden). Stock solutions of each peptide were prepared in water at a concentration of $10 \mathrm{mM}$. The standards were diluted in $50 \mathrm{mM} \mathrm{ABC}$ for further use.

Bovine serum albumin (BSA) and trypsin (TPCK-treated) from bovine pancreas (sequencing grade) were purchased from Sigma-Aldrich

Human Serum. Human serum from healthy individuals was obtained from Oslo University Hospital, Ullevål (Oslo, Norway). All serum samples were stored at $-32{ }^{\circ} \mathrm{C}$.

mMIP Synthesis. Two mMIP formats were synthesized: magnetic core-shell MIPs and magnetized MIP microspheres.

Magnetic core-shell MIPs were synthesized by a two-step precipitation polymerization (PP). For this, poly(MAA-coDVB-80) microspheres were synthesized and then magnetized in a first step, with these magnetic core particles then being used as seeds for the production of imprinted shells in a second 
precipitation polymerization. The magnetized MIP microspheres were prepared by the partial in-filling of the pores in MIP microspheres using a magnetic component. For the detailed synthesis of the polymers, see Supporting Information. Liquid Chromatography-Tandem Mass Spectrometry

LC-MS/MS analysis was performed using a triple quadrupole mass spectrometer according to established methods for ProGRP. ${ }^{27}$ The chromatographic system consisted of an LPG-3400 M pump with a degasser, a WPS-3000TRS autosampler, and an FLM3000 flow-manager (all Dionex, Sunnyvale, CA, USA). The LC system was controlled by Chromeleon v. 6.80 SR6 (Dionex). The chromatographic separation was carried out using an Aquasil C18 analytical column (Thermo Scientific) (100 ̊, $3 \mu \mathrm{m}, 50 \mathrm{~mm} \times 1 \mathrm{~mm})$. The chromatographic separation was performed by loading 10 $\mu \mathrm{L}$ of sample with mobile phase A (20 mM formic acid (FA) and acetonitrile $(\mathrm{MeCN}) 99: 1, \mathrm{v} / \mathrm{v}$ ) and eluting with a $30 \mathrm{~min}$ linear gradient from 0 to $85 \%$ mobile phase B (20 mM FA and $\mathrm{MeCN}$ 1:99, v/v). After the gradient was run, the column was washed for 3 min with $90 \%$ mobile phase B and re-equilibrated with mobile phase A. The column temperature was set and kept constant at $25^{\circ} \mathrm{C}$. A triple quadrupole mass spectrometer (TSQ Quantum Access, Thermo Scientific) was used to determine signature peptides by selected reaction monitoring (SRM). The following transition pairs were monitored: for the ProGRP signature peptide NLLGLIEAK, $485.8 \rightarrow 630.3$ and $485.8 \rightarrow 743.4$; for the NLLGLIEAK IS, $489.9 \rightarrow 638.3$ and $489.9 \rightarrow 751.4$; for the ProGRP signature peptide LSAPGSQR, $408.2 \rightarrow 272.6$ and $408.2 \rightarrow 544.4$; for the ProGRP signature peptide ALGNQQPSWDSEDSSNFK, $1005.450 \rightarrow$ 595.300, $1005.450 \rightarrow 913.300,1005.450 \rightarrow$ 1028.300 and $1005.450 \rightarrow 1398.500$. TSQ data were processed by Xcalibur's QualBrowser (version 2.2 SP 1.48, Thermo Scientific), and MS responses based on the peak intensity, automatically processed by genesis peak detection algorithm, were used. Among them, only peaks with a signalto-noise $(\mathrm{S} / \mathrm{N})$-ratio above 10 and with retention time and ion ratios corresponding to those of reference samples at high concentration were considered.

\section{Protein Digestion}

ProGRP standard solutions were diluted with $\mathrm{ABC}(50 \mathrm{mM})$ to a final concentration of $50 \mathrm{nM}$. Digestion was carried out with trypsin with an enzyme to substrate ratio of $1: 40$ at $37^{\circ} \mathrm{C}$, overnight.

BSA standards were diluted to a volume and concentration of $500 \mu \mathrm{L}$ and $100 \mathrm{nM}$, respectively, with $\mathrm{ABC}(50 \mathrm{mM}) .2 .5$ $\mu \mathrm{L}$ of $50 \mathrm{mM}$ DTT (freshly prepared in ABC buffer) was added to the protein mixture in $50 \mathrm{mM}$ freshly prepared $\mathrm{ABC}$ buffer and incubated at $800 \mathrm{rpm}$ at $60{ }^{\circ} \mathrm{C}$ for $20 \mathrm{~min}$. Afterward, the solution was cooled, and $2.5 \mu \mathrm{L}$ of $200 \mathrm{mM}$ IAA (freshly prepared in ABC buffer) was added. Incubation was carried out for $15 \mathrm{~min}$ at room temperature in the dark. Digestion was then accomplished by adding trypsin as described above.

\section{mMIP Preconditioning}

Prior to use, the mMIP was washed by gentle inversion overnight in 9:1 MeOH:HCl to remove any bound template. $\mathrm{MeOH}: \mathrm{HCl}$ was removed by washing twice with $\mathrm{MeCN}$ for 5 $\min$.

\section{Initial Testing of mMIPs}

The initial tests were performed on one batch of core-shell mMIP (mMIP A) to determine the requirements for conditioning, mass mMIP, extraction time and loading buffer (see Supporting Information for more details).

\section{Final Aqueous mSPE Protocol}

The mMIP was conditioned in $50 \mathrm{mM} \mathrm{ABC}(100 \mu \mathrm{L})$ before the addition of $100 \mu \mathrm{L}$ of loading buffer spiked with $5 \mathrm{nM}$ digested ProGRP, $5 \mathrm{nM}$ IS, and $10 \mathrm{nM}$ digested BSA and extracted for $5 \mathrm{~min}$. The supernatant was collected and the mMIP particles washed with $100 \mu \mathrm{L}$ Milli-Q $\mathrm{H}_{2} \mathrm{O}$ for 5 min. The bound peptides were eluted with $100 \mu \mathrm{L}$ 80:15:5 $\mathrm{H}_{2} \mathrm{O}$ :MeCN:FA for $5 \mathrm{~min}$. The eluent was dried under $\mathrm{N}_{2}$ and reconstituted in $100 \mu \mathrm{L} \mathrm{ABC}$ containing $0.1 \%$ FA. The eluent was analyzed by LC-MS/MS.

\section{Binding Isotherms}

mMIP C and its corresponding nonimprinted polymer (mNIP $\mathrm{C}$, i.e., a polymer synthesized under identical conditions to mMIP C except for the omission of template) were conditioned (as described in mMIP Preconditioning) before the addition of $100 \mu \mathrm{L}$ of loading buffer spiked with $5 \mathrm{nM}$ IS and $10 \mathrm{nM}$ digested BSA. After $5 \mathrm{~min}$, the supernatant was collected. This procedure was repeated for a total of $n=20$ with the same $\mathrm{mMIP} / \mathrm{mNIP}$ pair. The supernatants were analyzed to determine the binding profiles using the formula:

$$
\text { \%Bound }=100-\left(\frac{\mathrm{SI}_{\mathrm{EX}}}{\mathrm{SI}_{\mathrm{QC}}}\right) \cdot 100 \%
$$

where $\mathrm{SI}_{\mathrm{EX}}$ is the signal intensity from the supernatants after extraction, and $\mathrm{SI}_{\mathrm{QC}}$ is the mean of signal intensities from the QC-samples.

\section{Imprinting Factor (IF)}

Imprinting factors were determined using the ratio of the relative $B_{\max }$ (maximum specific binding) of the binding isotherms for the mMIP and mNIP, using the formula:

$$
\mathrm{IF}=\frac{B_{\max } \mathrm{mMIP}(\mathrm{nmol} / \mathrm{mg})}{B_{\max } \mathrm{mNIP}(\mathrm{nmol} / \mathrm{mg})}
$$

\section{Enrichment of NLLGLIEAK from Spiked Human Serum}

Human serum samples $(500 \mu \mathrm{L})$ were spiked to $10 \mathrm{nM}$ NLLGLIEAK IS and $10 \mathrm{nM}$ ProGRP, diluted 1:1 in $50 \mathrm{mM}$ $\mathrm{ABC}$ and vortexed for $30 \mathrm{~s}$. High molecular weight proteins were precipitated with $\mathrm{MeCN}$ at $-30{ }^{\circ} \mathrm{C}$ using a sample:MeCN ratio of 1:0.7. ${ }^{28}$ The precipitated proteins were removed by centrifugation $(10000 \mathrm{~g})$. Digestion was carried out with trypsin at a substrate to enzyme ratio of 1:20 (of calculated remaining protein concentration) at $37{ }^{\circ} \mathrm{C}$, overnight. The mMIP $(600 \mu \mathrm{g})$ was conditioned as described in mMIP Preconditioning and loaded with $100 \mu \mathrm{L}$ of digested sample. Extraction was performed for $5 \mathrm{~min}$. The mMIP was washed twice with $100 \mu \mathrm{L}$ of water. Peptides were eluted with $100 \mu \mathrm{L}$ 80:15:5 $\mathrm{H}_{2} \mathrm{O}: \mathrm{MeCN}$ :FA for $5 \mathrm{~min}$. The supernatant was then extracted 2 more times with fresh mMIP $(600 \mu \mathrm{g})$ to ensure maximum recovery. The eluents were pooled and dried under $\mathrm{N}_{2}$ and reconstituted in $50 \mathrm{mM} \mathrm{ABC}(100 \mu \mathrm{L})$ containing $0.1 \% \mathrm{FA}$ and analyzed LC-MS/MS. 
Table 1. Structural Information ${ }^{a}$ of the mMIPs and mNIPs

\begin{tabular}{|c|c|c|c|c|}
\hline & template & functional monomers & cross-linker & size $(\mu \mathrm{m})$ \\
\hline mMIP A & Z-NLLGLIEA[Nle] & EAMA·HCl, BTPV & DVB-80 & $4-5$ \\
\hline mNIP A & - & EAMA $\cdot \mathrm{HCl}, \mathrm{BTPV}$ & DVB-80 & $4-5$ \\
\hline mMIP B & Z-NLLGLIEA[Nle] & EAMA $\cdot \mathrm{HCl}$ & DVB-80 & $4-5$ \\
\hline mNIP B & - & EAMA $\cdot \mathrm{HCl}$ & DVB-80 & $4-5$ \\
\hline mMIP C & Z-NLLGLIEA[Nle] & EAMA.HCl & DVB-80 & $4-5$ \\
\hline mNIP C & - & EAMA $\cdot \mathrm{HCl}$ & DVB-80 & $1-5$ \\
\hline mMIP D & Z-NLLGLIEA[Nle] & EAMA.HCl, BTPV & DVB-80 & approximately 1 \\
\hline mNIP D & - & EAMA $\cdot \mathrm{HCl}, \mathrm{BTPV}$ & DVB-80 & approximately 1 \\
\hline
\end{tabular}

${ }^{a}$ For detailed information regarding concentrations and ratios of the synthetic components see Supporting Information: Tables S3 and S6.

\section{RESULTS AND DISCUSSION}

\section{Polymer Synthesis}

New approaches for the synthesis of magnetic MIPs and NIPs were developed, which allowed for the synthesis of imprinted and nonimprinted magnetic core-shell polymer microspheres (Synthesis Method 1) and imprinted and nonimprinted magnetic polymer microspheres (Synthesis Method 2). This outcome was achieved by adapting a literature protocol for microgel magnetization, and by drawing upon our extensive inhouse knowledge on polymer synthesis using precipitation polymerization (PP) and molecular imprinting. A noncovalent molecular imprinting strategy was adopted to impart affinity into selected polymers for the signature peptide of ProGRP, thereby building upon recent disclosures in this area. Precipitation polymerization was used as the polymer synthesis method of choice since it can deliver high quality polymer microspheres in the low-micron size range. A range of polymers was designed, synthesized, and then screened for their ability to recognize and bind to the target peptide in aqueous media followed by a magnetic capture; a list of the template, functional monomers and cross-linker used to prepare mMIPs and mNIPs is presented in Table 1 , together with a statement of the microsphere diameters. For full details about polymer synthesis and properties, see Supporting Information; however, the most salient points are outlined here.

Magnetic Core-Shell Polymer Microspheres (mMIP A, mNIP A, mMIP B, and mNIP B). The synthesis of mMIP $A$ and $\mathrm{mMIP} B$, and their corresponding NIPs, necessitated the synthesis of nonimprinted porous polymer microspheres bearing carboxylic acid groups (to enable the in-filling of pores with a magnetic component), thus poly(DVB-80-coMAA) microspheres with diameters $\sim 5 \mu \mathrm{m}$ were targeted. For this, PP conditions reported previously were applied. A monomer concentration of $3.28 \% \mathrm{w} / \mathrm{v}$ (with respect to the solvent) and an initiator concentration of $3.35 \mathrm{~mol} \%$ (with respect to the total number of moles of polymerizable double bonds), together with a mixture of acetonitrile and toluene as porogens $(75: 25(\mathrm{v} / \mathrm{v}))$, allowed for the synthesis of porous polymer microspheres of an appropriate size. Following the magnetization of these microspheres (see Supporting Information), they were used as seed particles in a subsequent PP. Accordingly, nonmagnetic shells were formed around the magnetic cores, taking advantage of the fact that the PP mechanism is one of nucleation and growth. A 2:1 w/w ratio of magnetic cores to monomer was used for the synthesis of the core-shell particles. Such a ratio allowed for the synthesis of core-shell polymer microspheres with shell thicknesses of $\sim 0.1 \mu \mathrm{m}$. MIPs (mMIP A and mMIP B) and the corresponding NIPs (mNIP A and mNIP B) were prepared by the delayed addition of template (for the MIP syntheses) and functional monomer(s), timed $1.5 \mathrm{~h}$ after the start of the PP.

Magnetic Polymer Microspheres (mMIP C, mNIP C, mMIP D, and mNIP D). mMIP C and mMIP D, and their corresponding NIPs, were prepared by magnetization of imprinted and nonimprinted porous polymer microspheres which had been produced via a PP protocol. Therefore, the first step was the synthesis of porous MIP microspheres (and their corresponding NIPs) with Z-NLLGLIEA[Nle] as template, which was followed by the magnetization procedure. For success, PP must involve the polymerization of monomers in dilute solution (typically $<5 \% \mathrm{w} / \mathrm{v}$ monomer in solvent) in a near- $\Theta$ solvent; therefore, DVB- 80 was selected as cross-linker, the porogen was acetonitrile, the initiator concentration was 2 mol \% (w.r.t. the total number of moles of polymerizable double bonds), and the monomer concentration was $2 \% \mathrm{w} / \mathrm{v}$ (w.r.t. to the solvent). A small volume of DMSO was required to promote solubility of template and keep all components in solution prior to polymerization, but the use of DMSO was kept to a minimum. $N$-(2-Aminoethyl)methacrylamide hydrochloride and $N-3,5-b i s$ (aminoethylmethyl)-phenyl- $N^{\prime}$-4-vinylphenylurea were selected as functional monomers since the carboxylic acid groups in the glutamic acid (E) residue and Cterminus of the template were targeted via a noncovalent molecular imprinting approach.

Overall, the polymer synthesis program delivered good yields of micron-sized imprinted and nonimprinted magnetic core-shell polymer microspheres (Synthesis Method 1) and imprinted and nonimprinted magnetic polymer microspheres (Synthesis Method 2), in a convenient beaded format. The magnetic susceptibility of the polymers meant that they could be used for the capture and quantification of an SCLC biomarker in a magnetic SPE platform.

\section{Selection of Standard Solutions}

Optimization of the mSPE method required an understanding of the optimal conditions for binding of the target by the mMIPs. For this, NLLGLIEAK IS was utilized in the initial optimization experiments as it circumvents the digestion step and simplifies sample preparation. The IS has chemical and chromatographic properties indistinguishable from native NLLGLIEAK but is distinct in $m / z(\Delta m=+8 \mathrm{Da})$. Synthetic NLLGLIEAK was incorporated in optimization experiments allowing IS correction. Furthermore, ProGRP was used for the evaluation of the final optimized aqueous extraction method. $50 \mathrm{mM} \mathrm{ABC}$ buffer was used to ensure compatibility with the increasing sample complexity in further optimization, such as 
tryptic digests, addition of digested BSA and finally digested ProGRP in serum.

Digested BSA was selected as the source of nonselectively bound competing peptides in the optimization of the mSPE protocol.

\section{Initial Testing}

Initial tests were carried out on mMIP A to determine the $\mathrm{mSPE}$ conditions (conditioning, loading matrix, extraction time, and mass of mMIP). Conditioning of the sorbent is essential for ensuring optimal interactions between the analyte and solid phase during extraction. Since the mMIPs are designed to enrich NLLGLIEAK from serum, the loading matrix should be aqueous to ensure downstream compatibility with tryptic digests. As such, the mMIP was loaded with the NLLGLIEAK IS $(5 \mathrm{ng} / \mathrm{mL})$ in ABC $(50 \mathrm{mM})$. Extractions of the target from an organic matrix $(100 \% \mathrm{MeCN})$ were also performed, since the mMIPs were synthesized in the presence of $\mathrm{MeCN}$ and therefore expected to show affinity for the target in this solvent. The binding efficiency (\% bound analyte) was found to be $99.9 \pm 0.0 \%$ and $99.9 \pm 0.3 \%$ in the aqueous and organic matrices, respectively (Figure S1). Therefore, the mMIPs were expected to have excellent compatibility with aqueous matrices and the potential to extract NLLGLIEAK directly from aqueous matrices such as serum.

Two essential aspects of mSPE optimization are the determination of an appropriate sorbent concentration and extraction time. Short extraction times are critical for low stability analytes, but also allow for a higher throughput of samples. The determination of optimal sorbent concentration is essential to ensure binding capacity is balanced against costeffectiveness. A range of mMIP concentrations and extraction times were explored to maximize the binding efficiency (Figure S2). This was accomplished by loading $5 \mathrm{nM}$ NLLGLIEAK IS $(100 \mu \mathrm{L})$ onto increasing amounts of mMIP $(200-600 \mu \mathrm{g})$ and extracting for between 10 and $120 \mathrm{~min}$. Supernatants were collected and analyzed directly to determine binding efficiency. The binding efficiency with $200 \mu \mathrm{g}$ mMIP was moderate between 10 and $40 \mathrm{~min}(25.4-38.4 \%)$, with high standard deviations for the shortest extraction times (10-30\%). Maximum binding efficiency of $91.0 \pm 4.6 \%$ was reached after $60 \mathrm{~min}$. Similarly, $400 \mu \mathrm{g}$ mMIP had moderate recoveries between 10 and $20 \mathrm{~min}$ with standard deviations from 7 to $23 \%$; however, $92.8 \pm 2.2 \%$ of NLLGLIEAK IS was bound after $50 \mathrm{~min}$. With $600 \mu \mathrm{g} \mathrm{mMIP}$, there was consistent, high binding efficiency from the earliest time point (10 min; $92.3 \pm$ $2.8 \%$ ), with up to $99.5 \%$ of the peptide being bound from 50 to $120 \mathrm{~min}$. Accordingly, all further experiments were performed using $600 \mu \mathrm{g}$ of mMIP and $100 \mu \mathrm{L}$ of sample (i.e., $6 \mathrm{mg}$ mMIP per $\mathrm{mL}$ sample) since this gave high binding of the target within short incubation times.

\section{mMIP Evaluation}

The molecular recognition properties of the mMIPs were evaluated by investigating their binding strength and selectivity compared to their mNIP counterparts. The performance of all $\mathrm{mMIP} / \mathrm{mNIP}$ pairs (mMIP/mNIP A-D) was assessed by determining their binding efficiencies via extraction of the NLLGLIEAK IS (5 $\mathrm{nM})$ from ABC $(50 \mathrm{mM})$ containing 10 $\mathrm{nM}$ digested BSA. BSA $(10 \mathrm{nM})$ was included to model a potential source of nonspecific binding from endogenous proteins, to illustrate selectivity while maintaining a simple matrix. To evaluate binding, the supernatant was measured directly; therefore, serum equivalent levels of BSA are impractical. While considerably lower than serum levels of albumin were used, a 2-fold concentration of BSA compared to NLLGLIEAK ought to allow influence on binding selectivity to be determined. Under the conditions of the extraction, mMIP $\mathrm{C}$ was found to have particularly high affinity and selectivity for the target (Figure 1), which suggested that mMIP C was an

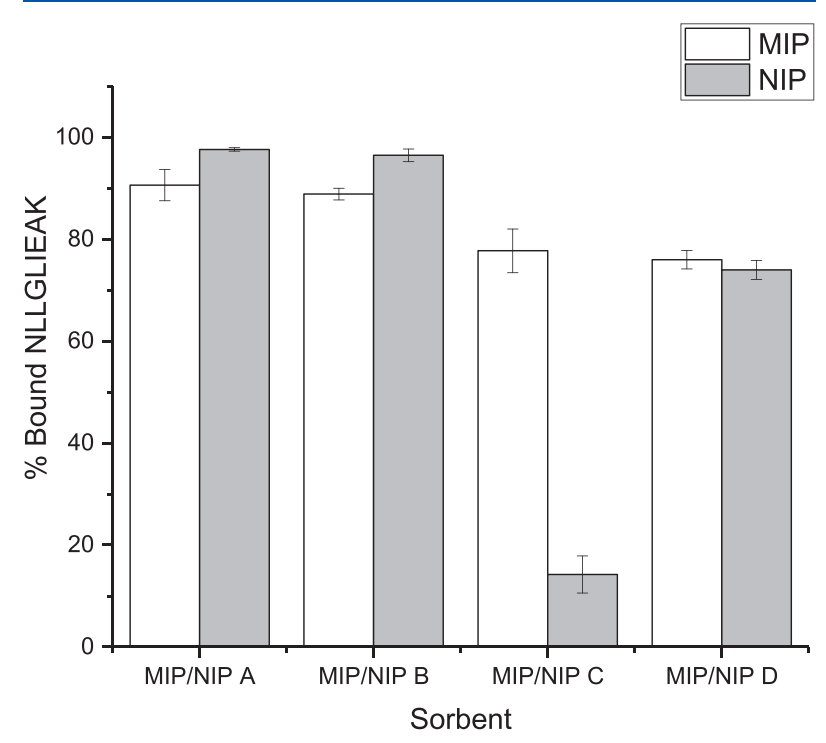

Figure 1. Selectivity of the $\mathrm{mMIP} / \mathrm{mNIP}$ pairs toward target peptide determined as binding efficiency (\% bound NLLGLIEAK IS \pm standard deviation of NLLGLIEAK IS). Samples consisted of NLLGLIEAK IS $(5 \mathrm{nM})$ in $\mathrm{ABC}(50 \mathrm{mM})$ containing $10 \mathrm{nM}$ digested BSA $(n=3)$.

excellent candidate for use with complex matrices where both affinity and selectivity are important criteria. ${ }^{29}$ The other $\mathrm{mMIP} / \mathrm{mNIP}$ pairs showed high affinity for the target as well, but poor selectivity under the conditions of the test, therefore mMIP C was selected as the mMIP to be used in the subsequent experiments. It is noteworthy that mMIP $\mathrm{C}$ was expected to have higher selectivity than any of the core-shell materials, and was synthesized using a functional monomer (EAMA $\cdot \mathrm{HCl}$ ) which gave rise to high fidelity binding sites for NLLGLIEAK in our earlier published work on online MISPE; this is why mMIP C outperforms the other MIPs.

\section{Binding Isotherms}

Binding isotherms give a broader picture with respect to single concentration extractions of the molecular recognition capabilities of MIPs across a range of concentrations, and were constructed for the $\mathrm{mMIP} / \mathrm{mNIP} \mathrm{C}$ pair for binding to NLLGLIEAK. The nonlinear shape of the mMIP curve (Figure 2 ) is indicative of selective binding of the target molecule to the molecularly imprinted binding sites in the MMIP, whereas the plot for mNIP C is typical of a situation where binding of the target to the polymer is nonselective in nature. Saturation was reached for the mMIP after 13 extractions, with a $B_{\max }$ of $7.4 \mathrm{pmol}$ NLLGLIEAK/mg mMIP (Figure 2). The dissociation constant $\left(K_{\mathrm{d}}\right)$ for mMIP C was calculated to be $2.18 \times$ $10^{-9} \mathrm{M}$. Values of $K_{\mathrm{d}}$ in the low nanomolar range (as are observed here) indicates high affinity between mMIP $\mathrm{C}$ and NLLGLIEAK, and is in line with the $K_{d}$ ranges observed for antigen-antibody binding. 


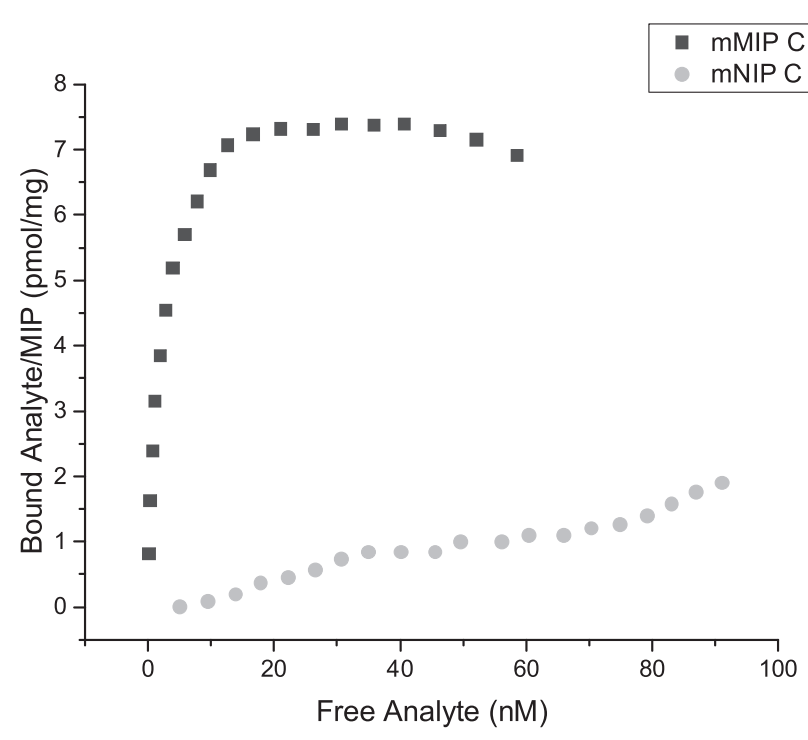

Figure 2. Binding isotherms for mMIP C and $\mathrm{mNIP} C$, expressed as bound analyte/mg mMIP or mNIP vs concentration of free analyte. Samples consisted of NLLGLIEAK IS (5 nM) in ABC (50 mM) containing $10 \mathrm{nM}$ digested BSA $(n=2)$.

\section{Imprinting Factor}

A measure of the efficiency of a molecular imprinting process can be gained by determination of the imprinting factor (IF), wherein the binding of an analyte to a MIP is compared to the binding of the same analyte to a polymeric control under nominally identical conditions. While the IF for a MIP does not have a fixed value-since the value measured depends on a number of factors, including the balance of selective and nonselective binding to the MIP under the conditions of the measurement-higher values indicate that there are conditions under which selective binding of an analyte to a MIP can be realized and potentially exploited. In the present case, the IF of mMIP C was calculated to be 6.1, which gave us confidence that molecular imprinting was successful and that binding conditions had been identified under which NLLGLIEAK could be extracted selectively from aqueous media. By comparison, other magnetic MIPs targeting the peptides angiotensin I and angiotensin II were reported to have IFs of 4.9 and 5.2, respectively. ${ }^{12}$ Furthermore, an epitope imprinted nanogel for human serum transferrin (HTR) had a similar IF $(5.49) .{ }^{21}$ Since IF is an indicator of imprinting efficiency, ${ }^{29}$ the higher the IF the more likely it is that the MIP will be able to discriminate between the target peptide and nontarget peptides during extractions involving complex matrices such as serum. Optimization of the mSPE Method

With mMIP C having been identified as the most promising polymer, the mSPE protocol was optimized further with MMIP $\mathrm{C}$ to ensure that a robust protocol was in place for the extraction of target peptide from serum. This involved optimization of the loading, washing, and elution steps using synthetic NLLGLIEAK (and NLLGLIEAK IS) in $50 \mathrm{mM} \mathrm{ABC}$ containing digested BSA.

Sample Loading. The sample loading procedure was finetuned for mMIP C. NLLGLIEAK ( $5 \mathrm{nM}$ ), NLLGLIEAK IS (5 $\mathrm{nM})$, and digested BSA $(10 \mathrm{nM})$ were spiked in $50 \mathrm{mM} \mathrm{ABC}$ with increasing $\mathrm{MeCN}(0-10 \%)$. mMIP C $(6 \mathrm{mg} / \mathrm{mL})$ was added, and the samples agitated for an hour. Following magnetic capture of mMIP C, the supernatants were analyzed to determine the binding efficiency. The binding efficiency was highest under fully aqueous conditions ( $50 \mathrm{mM} \mathrm{ABC}$ ), with $98.9 \pm 0.2 \%$ NLLGLIEAK bound. The introduction of small amounts of $\mathrm{MeCN}$ reduced the binding efficiency; for $2.5 \%$ $\mathrm{MeCN}$, the binding efficiency dropped to $91.6 \pm 7.3 \%$, whereas further increases in $\mathrm{MeCN}$ levels resulted in large variations in binding efficiency (RSD > 100\%). This data shows that mMIP C functioned very well in aqueous media, even when in the presence of nontarget peptides (digested BSA), and is well-suited for compatibility with complex matrix $\mathrm{mSPE}$ because the conditions in digested serum are aqueous. All subsequent extractions were performed in $100 \%$ aqueous media to ensure downstream compatibility with serum extractions and ensure good repeatability.

Extraction Time. The extraction time was evaluated to determine the shortest extraction time possible while still retaining a high level of binding of NLLGLIEAK. NLLGLIEAK ( $5 \mathrm{nM})$, NLLGLIEAK IS ( $5 \mathrm{nM}$ ), and digested BSA $(10 \mathrm{nM})$ were spiked in $50 \mathrm{mM} \mathrm{ABC}$, and a $100 \mu \mathrm{L}$ sample extracted for 5-60 min; following magnetic separation, the supernatant was analyzed to determine the dependence of the extraction time on the binding efficiency. It was found that mMIP C was able to bind NLLGLIEAK efficiently (98.2 \pm $0.2 \% ; n=3$ ) in just $5 \mathrm{~min}$ (Figure 3 ). The results show that

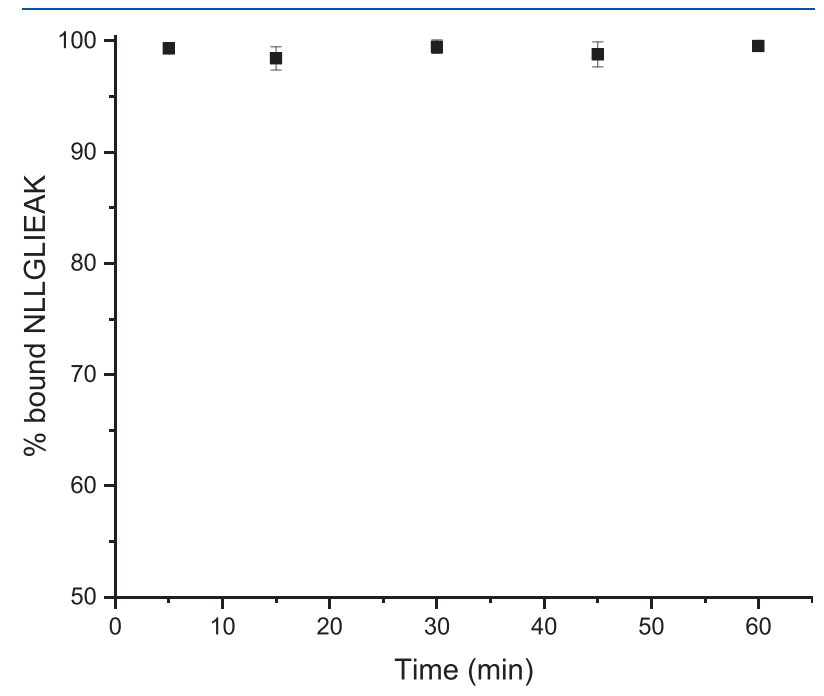

Figure 3. Effect of increasing the extraction time on the binding efficiency ( $\%$ bound NLLGLIEAK \pm standard deviation) of NLLGLIEAK using mMIP C. Samples consisted of NLLGLIEAK IS ( $5 \mathrm{nM})$, NLLGLIEAK $(5 \mathrm{nM})$, and $10 \mathrm{nM}$ digested BSA in $50 \mathrm{mM}$ ABC. Samples were extracted for 5, 15, 30, 45, and $60 \mathrm{~min}(n=3)$.

mMIP C can extract NLLGLIEAK with high recovery using short extraction times ( $5 \mathrm{~min})$. Short extraction times are particularly advantageous if the targets have low stability at room temperature, but they also facilitate high sample throughput.

Washing Step. Next, the washing step was optimized. Washing of the mMIP is essential to remove nonspecifically bound peptides, and other adsorbed components, from the polymer prior to elution to ensure a clean extract for analysis. Care must be taken to avoid loss of the target peptide during washing, and a compromise may have to be struck between the loss of target peptide and efficient removal of adsorbed 

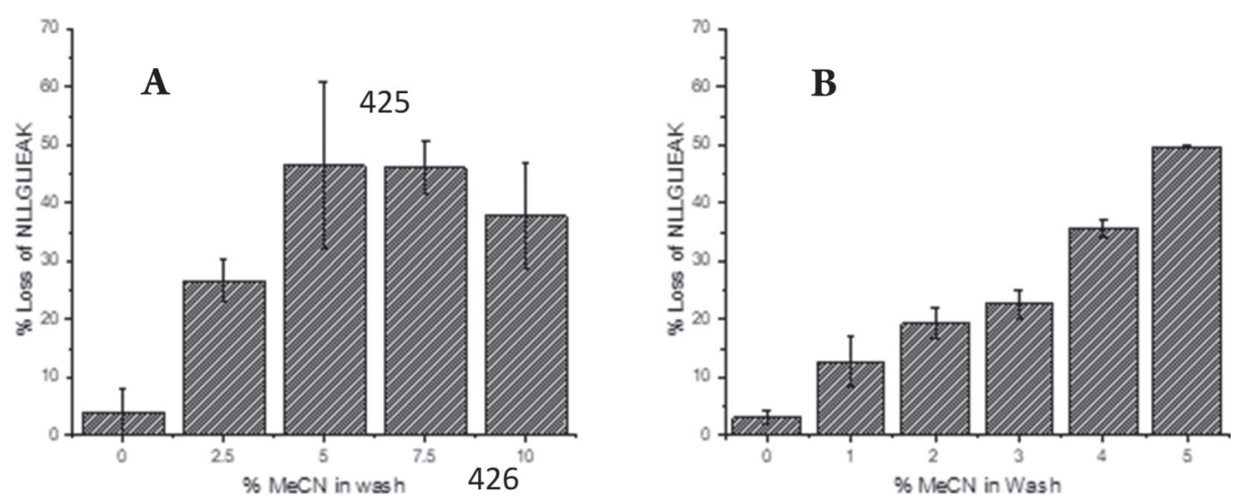

Figure 4. Effect of increasing MeCN in the wash buffer on the loss of NLLGLIEAK (\% loss NLLGLIEAK \pm standard deviation) of NLLGLIEAK using mMIP C. Samples consisted of NLLGLIEAK IS ( $5 \mathrm{nM})$, NLLGLIEAK $(5 \mathrm{nM})$, and $10 \mathrm{nM}$ digested BSA in $50 \mathrm{mM} \mathrm{ABC}$, and were extracted for $5 \mathrm{~min}$. (A) Samples were washed with buffers containing 0, 2.5, 5, 7.5, and 10\% $\mathrm{MeCN}(n=3)$. (B) Fine-tune washing using $0,1,2,3,4$, and $5 \% \operatorname{MeCN}(n=3)$.

compounds. To identify an optimal wash buffer, NLLGLIEAK ( $5 \mathrm{nM})$, NLLGLIEAK IS (5 $\mathrm{nM})$, and digested BSA (10 $\mathrm{nM})$ were spiked in $50 \mathrm{mM} \mathrm{ABC}$ and $100 \mu \mathrm{L}$ samples extracted for $5 \mathrm{~min}$. The mMIPs were then washed in buffers containing increasing concentrations of $\mathrm{MeCN}(0,2.5,5,7.5$, and $10 \%)$. The wash time was set to $5 \mathrm{~min}$ to ensure a short sample preparation time and to minimize any loss of the target peptide. As can be seen in Figure 4a, the general trend is that more NLLGLIEAK is lost as the $\mathrm{MeCN}$ content of the washing solution rises (this is in agreement with the sample loading findings). Considerable losses ( $>35 \%$ ) were observed using 5, 7.5 and $10 \% \mathrm{MeCN}$ in the wash solution, together with high standard deviations (RSD $\geq 24 \%$ ) for 5 and $10 \%$ $\mathrm{MeCN}$. However, there was minimal loss of target peptide (2.2 $\pm 1.6 \%$ ) using a $100 \%$ aqueous wash solution. Since the differences in loss of target were so large between 0 and $5 \%$ $\mathrm{MeCN}, \mathrm{MeCN}$ contents ranging from 0 to $5 \%$ were evaluated as well; the results are shown in Figure $4 \mathrm{~b}$. A similar trend was observed, in that the amount of target lost was directly proportional to the amount of the $\mathrm{MeCN}$ in the wash buffer. As there were significant losses at even minor increments of $\mathrm{MeCN}$, it was decided that no consideration would be made with regards to removal of nonspecific peptides. Given all of these results, a fully aqueous washing buffer was selected for use in the subsequent experiments.

Elution of Target Peptide. The final stage of the mSPE procedure is the elution of the target peptide from the polymer using an elution buffer. Elution efficiency (determined as the \% recovery) was evaluated using $\mathrm{mMIP} C$ with NLLGLIEAK (5 $\mathrm{nM})$, NLLGLIEAK IS (5 nM), and digested BSA (10 nM) spiked in $50 \mathrm{mM} \mathrm{ABC}$. The sample $(100 \mu \mathrm{L})$ was extracted for 5 min with mMIP C and was then washed with water $(100 \mu \mathrm{L})$ for $5 \mathrm{~min}$. First, two eluents were evaluated based on the outcomes of the earlier wash experiments: one eluent was 7.5:92.5 $\mathrm{MeCN}: \mathrm{H}_{2} \mathrm{O}$ and the other was 7.5:92.5 MeCN:0.1\% FA in $\mathrm{H}_{2} \mathrm{O}$. FA was included as a component in one of the eluents since acidic conditions were expected to disrupt the noncovalent interactions between the functional monomers EAMA $\cdot \mathrm{HCl}$ and BTPV of mMIP C and NLLGLIEAK. In the washing experiments, $7.5 \% \mathrm{MeCN}$ in $\mathrm{ABC}$ led to approximately 50\% loss of NLLGLIEAK; however, when used with water as an eluent it gave rise to low and variable recoveries $(2.5 \pm 4.3 \%$; Table 2$)$. Furthermore, acidifying the eluent with a low level of FA gave a marginal improvement in recovery
Table 2. Recoveries of NLLGLIEAK after Elution with a Range of Eluents, as Represented by \% Recovery NLLGLIEAK \pm Standard Deviation ${ }^{a}$

\begin{tabular}{|ccc|}
\hline eluent & recovery (\%) & RSD (\%) \\
\hline 7.5:92.5 MeCN: $\mathrm{H}_{2} \mathrm{O}$ & 2.5 & 173 \\
7.5:92.5 MeCN: $\mathrm{H}_{2} \mathrm{O}(0.1 \% \mathrm{FA})$ & 6.2 & 173 \\
80:15:5 MeCN: $\mathrm{H}_{2} \mathrm{O}: \mathrm{FA}$ & 84.8 & 14.1
\end{tabular}

${ }^{a}$ Samples consisted of NLLGLIEAK ( $5 \mathrm{nM}$ ) and NLLGLIEAK IS (5 $\mathrm{nM})$ in $\mathrm{ABC}(50 \mathrm{mM})$ containing $10 \mathrm{nM}$ digested BSA. Samples were extracted for $5 \mathrm{~min}$, washed in $50 \mathrm{mM} \mathrm{ABC}(100 \mu \mathrm{L})$ for $5 \mathrm{~min}$ and eluted for $5 \min (n=3)$.

only $(6.2 \pm 10.7 \%)$. A more potent eluent (80:15:5 $\mathrm{MeCN}: \mathrm{H}_{2} \mathrm{O}: \mathrm{FA}$ ) was therefore evaluated, an eluent which had a high organic content (to promote efficient wetting of the polymer and solubilization of the bound target) and a higher FA content (to break selective interactions); in earlier work, this eluent had been used successfully to elute NLLGLIEAK from imprinted polymers. ${ }^{30}$ With this eluent, the recovery was markedly increased to $84.8 \%$, and with a satisfactory RSD $(<15 \%)$ (Table 2). 80:15:5 MeCN: $\mathrm{H}_{2} \mathrm{O}: \mathrm{FA}$ was hence selected as the preferred eluent for the remainder of the experiments.

\section{Affinity of mMIPs toward Other Peptides}

To evaluate the effectiveness and selectivity of the optimized mSPE method, the whole procedure was performed using digested ProGRP $(250 \mathrm{ng} / \mathrm{mL})$ in ABC $(50 \mathrm{mM})$. Each step in the procedure was evaluated: binding efficiency, loss in washing, and elution recovery. Three peptides were monitored: the target peptide, NLLGLIEAK, and two other ProGRP isoform 1 peptides, ALGNQQPSWDSEDSSNFK and LSAPGSQR. In these experiments, binding efficiency was determined as the normalized amount of peptide in the supernatant recovery (i.e., ratio of the amount of peptide measured in the supernatant and amount of peptide measured in the control, where a low supernatant recovery suggests efficient binding to the mMIP). LSAPGSQR bound poorly to mMIP $C$, with $75.6 \pm 10.6 \%$ unbound after incubation with the sample, however ALGNQQPSWDSEDSSNFK bound strongly to mMIP C. The latter observation can be explained on the basis that ALGNQQPSWDSEDSSNFK contains carboxylate side-chains that can bind strongly but nonselectively to amine moieties throughout the polymer. 
Unsurprisingly, the target peptide, NLLGLIEAK, also binds strongly to mMIP C when extracting from a digested ProGRP sample (Figure 5).

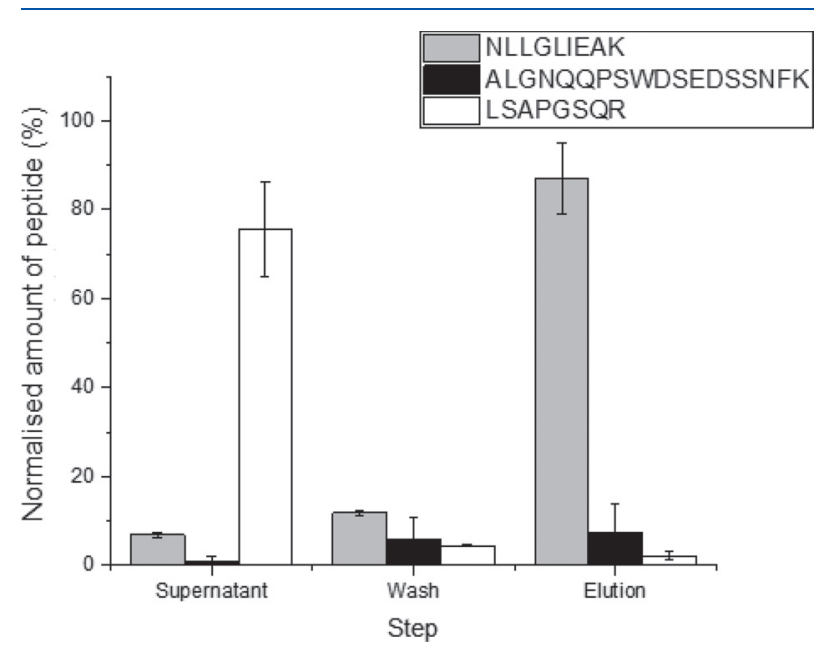

Figure 5. Evaluation of the selectivity of each step in the mSPE method using digested ProGRP, as represented by normalized amount of peptide (\%) \pm standard deviation of three ProGRP peptides for the three steps. Samples consisted of ProGRP (182 nM) and NLLGLIEAK IS $(5 \mathrm{nM})$ in $\mathrm{ABC}(50 \mathrm{mM})$ containing $10 \mathrm{nM}$ digested BSA. Samples were extracted for $5 \mathrm{~min}$, washed in $50 \mathrm{mM}$ $\mathrm{ABC}(100 \mu \mathrm{L})$ for $5 \mathrm{~min}$ and eluted with $80: 15: 5 \mathrm{MeCN}: \mathrm{H}_{2} \mathrm{O}$ :FA for $5 \min (n=3)$.

The wash fraction had normalized amounts of LSAPGSQR and ALGNQQPSWDSEDSSNFK of $4.5 \pm 0.7 \%$ and $5.9 \pm$ $4.4 \%$ respectively. For NLLGLIEAK in the wash this was 11.8 $\pm 0.6 \%$.

The normalized amounts of LSAPGSQR and ALGNQQPSWDSEDSSNFK in the elution step (i.e., elution recovery) were poor, with an elution recovery of $7.5 \pm 6.5 \%$ and $2.2 \pm$ $1.0 \%$ respectively. However, NLLGLIEAK had an elution recovery of $87 \pm 8.1 \%$, showing, under these conditions, mMIP C's selectivity toward NLLGLIEAK compared to LSAPGSQR and ALGNQQPSWDSEDSSNFK as NLLGLIEAK is eluted almost quantitatively off mMIP C. The differences in elution between the peptides are likely to be due to differences in their physicochemical properties. The size (i.e., molecular weight), hydrophobicity (i.e., grand average of hydrophobicity, GRAVY) and isoelectric points (pI) of the tightly bound peptides are quite different: ALGNQQPSWDSEDSSNFK has a $M_{\mathrm{w}}$ of $2010.06 \mathrm{Da}$, GRAVY of -1.450 and a pI of 3.68, while NLLGLIEAK has a $M_{w}$ of $970.18 \mathrm{Da}$, GRAVY of 0.711 and a pI of 6.00. Therefore, under the elution conditions (approximately $\mathrm{pH} 2$ ), the acidic groups of NLLGLIEAK will be protonated, disrupting the interactions with the functional groups in the polymer. ALGNQQPSWDSEDSSNFK, on the other hand, has a pI of 3.68 and is, therefore, more likely to remain bound to EAMA. Furthermore, since NLLGLIEAK is less polar than ALGNQQPSWDSEDSSNFK it will have a higher affinity for an eluent with a high $\mathrm{MeCN}$ content. A consequence of ALGNQQPSWDSEDSSNFK remaining bound to MMIP C after the elution step there may be interferences with the binding of NLLGLIEAK to mMIP $\mathrm{C}$ in subsequent extractions. To mitigate this, it would be advisable to perform a thorough wash step before reuse. This wash step should be similar to the initial particle wash protocol, as described in mMIP Preconditioning. This would limit the reuse time to once every day; however, the reusability of the mMIPs ensures lowcost analysis.

\section{Applicability to Complex Matrices}

To round-off the study, mMIP C was applied to the mSPE of a real biological sample, specifically a human serum sample containing the biomarker ProGRP. For this, serum was spiked with ProGRP and NLLGLIEAK IS before precipitation of the high molecular weight proteins, as described previously. ${ }^{7,26}$ After protein precipitation, evaporation, and reconstitution, the serum was digested and $\mathrm{mSPE}$ performed using the optimized method. Initially, the recovery of the target for this extraction of a complex matrix was low $(5.6 \pm 0.5 \%$; Figure 6$)$. This is

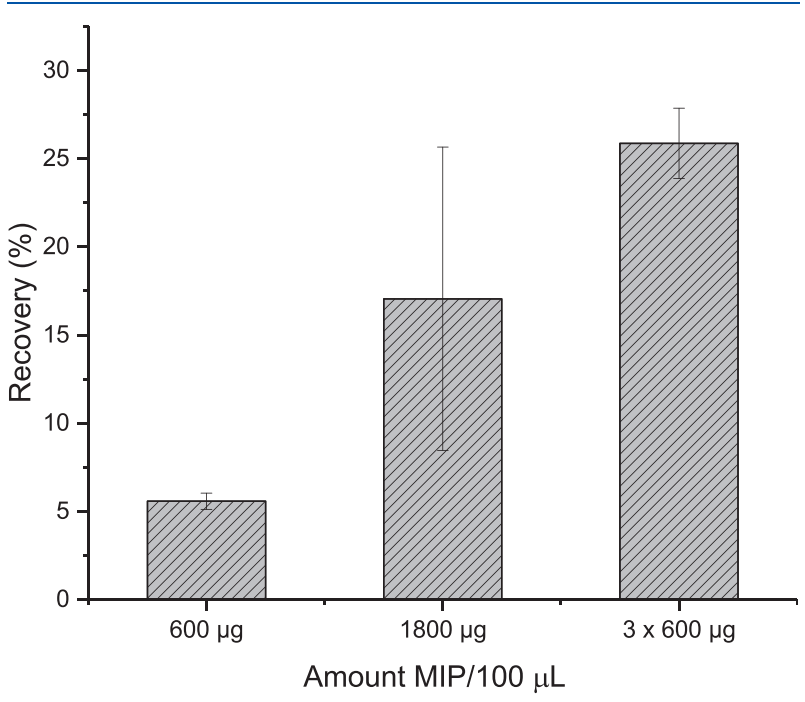

Figure 6. Recoveries of NLLGLIEAK from human serum using digested ProGRP, as represented by \% recovery NLLGLIEAK \pm standard deviation. Samples consisted of ProGRP (10 nM) and NLLGLIEAK IS (10 nM) in $50 \mu \mathrm{L}$ serum diluted 1:1 in ABC (50 $\mathrm{mM}$ ). Serum was digested with trypsin and samples were extracted for 5 min with $600 \mu \mathrm{g}, 1800 \mu \mathrm{g}$ and $3 \times 600 \mu \mathrm{g}$ mMIP C/ $100 \mu \mathrm{L}$ sample, samples were washed in $50 \mathrm{mM} \mathrm{ABC}(100 \mu \mathrm{L})$ for 5 min and eluted with 80:15:5 MeCN: $\mathrm{H}_{2} \mathrm{O}: \mathrm{FA}$ for $5 \mathrm{~min}(n=3)$.

most likely due to the high abundant, nontarget peptides binding nonselectively to the mMIP binding sites and preventing NLLGLIEAK capture, which suggests capacity limitations, i.e., too few binding sites, an effect that has been described previously. ${ }^{25}$ Furthermore, the complexity of serum can limit the digestion efficiency, thereby also lowering the recovery of target. The volume of extracted serum was $50 \mu \mathrm{L}$, diluted 1:1 in $50 \mathrm{mM} \mathrm{ABC}$, and low sample volumes can present challenges with recoveries and LODs. To improve the recovery of the process, an increase in the mass of mMIP C used (1800 $\mu \mathrm{g} \mathrm{mMIP} \mathrm{C} / 100 \mu \mathrm{L}$ sample) and sequential extractions using $3 \times 600 \mu \mathrm{g} \mathrm{mMIP} \mathrm{C} / 100 \mu \mathrm{L}$ sample were explored. The use of a higher amount of polymer increased the recovery to $17.1 \pm 8.6 \%$, and the use of sequential extraction further increased the recovery to $25.9 \pm 2.0 \%$. While both methods used a total of $1800 \mu \mathrm{g}$ of mMIP C, the sequential extractions yielded higher recoveries and lower variation. This increased recovery is in accordance with conventional extraction theory (e.g., for liquid-liquid extractions). 
A recovery of $25 \%$ is comparable to a recovery reported for nonmagnetic MIPs ${ }^{25}$ as well as antibody-based cleanup of low abundant proteins in human serum. ${ }^{31}$ This is considered to be satisfactory if the method otherwise provides repeatable and accurate results and at sufficiently low detection and quantification limits.

An estimate of the detection and quantification limits (LOD and LOQ respectively) was carried out based on the signal intensity of NLLGLIEAK after analysis of the spiked serum sample. $\mathrm{LOD}(\mathrm{S} / \mathrm{N}=3)$ and LOQ $(\mathrm{S} / \mathrm{N}=10)$ were estimated to be $39 \mathrm{pM}$ and $129 \mathrm{pM}$, respectively. This is significantly lower than the LOD reported for crushed and ground MIP particles packed into SPE-cartridges (LOD $625 \mathrm{pM})^{25}$ and of the same order of magnitude as reported for MIP microparticles applied in online SPE (LOD $11 \mathrm{pM}){ }^{7}$ The observed LOD is 5.6 times higher than the upper reference level for humans in humans, ${ }^{24}$ but this should be within reach after further optimization of the mSPE method and/or use of a more sensitive LC-MS/MS system. The recovery is most likely affected by two factors: limited binding capacity and interference from matrix components. In respect of interference from matrix components, the mMIP is likely to interact with many abundant tryptic peptides in the matrix, as has been observed previously for MIPs with similar compositions targeting NLLGLIEAK. ${ }^{7,32}$ It is expected that use of mMIPs with higher binding capacities will yield higher recoveries despite nonspecific interactions of the matrix components (N.B. mMIP C was synthesized using a template to cross-linker mole ratio in the feed of 1:533, thus there is significant scope for preparing mMIPs with significantly higher binding capacities, if desired, by increasing the template to cross-linker ratio during the polymer synthesis stage).

\section{CONCLUSIONS}

In the present study, four magnetic synthetic receptors (mMIPs) were synthesized in two distinct beaded formats, magnetic polymer microspheres, and magnetic core-shell polymer microspheres, using a simple and straightforward magnetization procedure that can be applied to a range of porous media, nonrestricted to beaded materials. The magnetization of the synthetic receptors enabled them to be evaluated for the targeting of the signature peptide of the SCLC biomarker, ProGRP, using a magnetic SPE (mSPE) platform coupled with LC-MS/MS for bottom-up proteomics. The binding selectivity of each mMIP was assessed to determine the most promising $\mathrm{mMIP}$ for the optimization of the mSPE method, with one imprinted material (mMIP C) displaying particularly high fidelity for the target, even in fully aqueous media. In this regard, a dissociation constant in the low nanomolar range was estimated for mMIP C which, when taken together with its magnetic character, enabled an optimized mSPE protocol to be established to selectively cleanup NLLGLIEAK from a digested ProGRP sample. Extractions of the biomarker from digested serum samples were also possible, with satisfactory repeatability, which demonstrated the applicability of the mMIP platform to real samples. Sample volumes were low, high recoveries were obtained within very short extraction times $(5 \mathrm{~min})$, and the LOD was $39 \mathrm{pM}$ (this LOD is significantly lower than the LOD reported for crushed and ground MIP particles packed into SPE-cartridges). With further optimization and testing, these mMIPs may have potential in clinical settings given their high selectivity and good recoveries at a much lower price point than conventional methods.

\section{ASSOCIATED CONTENT}

Supporting Information

The Supporting Information is available free of charge at https://pubs.acs.org/doi/10.1021/acs.jproteome.0c00258.

Figures showing initial optimization of mMIP extraction; supplementary results and discussion of mMIP and mNIP production including figures and tables showing production conditions and particle characterization; overview of raw data files uploaded to Peptide Atlas (PDF)

Additional data (XLSX)

\section{AUTHOR INFORMATION}

\section{Corresponding Authors}

Peter A. G. Cormack - WestCHEM, Department of Pure and Applied Chemistry, University of Strathclyde, Glasgow G1 IXL, Scotland, U.K.; Email: peter.cormack@strath.ac.uk

Trine Grønhaug Halvorsen - Section for Pharmaceutical Chemistry, Department of Pharmacy, University of Oslo, 0316 Oslo, Norway; @ orcid.org/0000-0002-2545-3474; Email: trinegha@farmasi.uio.no, t.g.halvorsen@ farmasi.uio.no

\section{Authors}

Nicholas McKitterick - Section for Pharmaceutical Chemistry, Department of Pharmacy, University of Oslo, 0316 Oslo, Norway

Frida Braathen - Section for Pharmaceutical Chemistry, Department of Pharmacy, University of Oslo, 0316 Oslo, Norway

Magdalena A. Switnicka-Plak - WestCHEM, Department of Pure and Applied Chemistry, University of Strathclyde, Glasgow G1 1XL, Scotland, U.K.

Léon Reubsaet - Section for Pharmaceutical Chemistry, Department of Pharmacy, University of Oslo, 0316 Oslo, Norway; i orcid.org/0000-0001-5165-2701

Complete contact information is available at:

https://pubs.acs.org/10.1021/acs.jproteome.0c00258

Notes

The authors declare no competing financial interest.

\section{ACKNOWLEDGMENTS}

This work was supported by the Horizon 2020 Research Framework Programme and the 7th Research Framework Programme of the European Commission, BioCapture project (722171) and PEPMIP (264699), respectively. We thank Mingquan Liu (Umeå University, Umeå, Sweden) for assisting with the synthesis of MIPs, Cecilia Rossetti (University of Oslo, Oslo, Norway) for initial MIP evaluation and Frederik Hansen (University of Oslo, Oslo, Norway) for giving valuable feedback on the manuscript. Elisabeth Paus and Marianne N Broughton (Oslo University Hospital, Oslo, Norway) are acknowlegded for the kind gift of recominant ProGRP.

\section{REFERENCES}

(1) Li, J.; Zhang, Z.; Rosenzweig, J.; Wang, Y. Y.; Chan, D. W. Clin. Chem. 2002, 48, 1296-1304. 
(2) Echan, L. A.; Tang, H. Y.; Ali-Khan, N.; Lee, K.; Speicher, D. W. Proteomics 2005, 5, 3292-3303.

(3) Zhang, Y.; Fonslow, B. R.; Shan, B.; Baek, M.-C.; Yates, J. R., III Chem. Rev. 2013, 113, 2343-2394.

(4) Haupt, K.; Mosbach, K. Chem. Rev. 2000, 100, 2495-2504.

(5) Mujahid, A.; Iqbal, N.; Afzal, A. Biotechnol. Adv. 2013, 31, 14351447.

(6) Poma, A.; Turner, A. P.; Piletsky, S. A. Trends Biotechnol. 2010, $28,629-637$.

(7) Rossetti, C.; Levernaes, M. C.; Reubsaet, L.; Halvorsen, T. G. J. Chromatogr. A 2016, 1471, 19-26.

(8) Li, M. X.; Wang, X. H.; Zhang, L. M.; Wei, X. P. Anal. Biochem. 2017, 530, 68-74.

(9) Hilt, J. Z.; Byrne, M. E. Adv. Drug Delivery Rev. 2004, 56, 15991620.

(10) Saridakis, E.; Khurshid, S.; Govada, L.; Phan, Q.; Hawkins, D.; Crichlow, G. V.; Lolis, E.; Reddy, S. M.; Chayen, N. E. Proc. Natl. Acad. Sci. U. S. A. 2011, 108, 11081-11086.

(11) Chen, L.; Wang, X.; Lu, W.; Wu, X.; Li, J. Chem. Soc. Rev. 2016, $45,2137-2211$.

(12) Tan, L.; Yu, Z.; Zhou, X.; Xing, D.; Luo, X.; Peng, R.; Tang, Y. J. Chromatogr. A 2015, 1411, 69-76.

(13) Zhou, W.-H.; Lu, C.-H.; Guo, X.-C.; Chen, F.-R.; Yang, H.-H.; Wang, X.-R. J. Mater. Chem. 2010, 20, 880-883.

(14) Miao, S. S.; Wu, M. S.; Zuo, H. G.; Jiang, C.; Jin, S. F.; Lu, Y. C.; Yang, H. J. Agric. Food Chem. 2015, 63, 3634-3645.

(15) Chen, L. G.; Li, B. Anal. Methods 2012, 4, 2613-2621.

(16) Huang, S.; Xu, J.; Zheng, J.; Zhu, F.; Xie, L.; Ouyang, G. Anal. Bioanal. Chem. 2018, 410, 3991-4014.

(17) Zhang, Z.; Yang, X.; Chen, X.; Zhang, M.; Luo, L.; Peng, M.; Yao, S. Anal. Bioanal. Chem. 2011, 401, 2855.

(18) Gai, Q. Q.; Qu, F.; Liu, Z. J.; Dai, R. J.; Zhang, Y. K. J. Chromatogr. A 2010, 1217, 5035-5042.

(19) Tan, C. J.; Tong, Y. W. Anal. Chem. 2007, 79, 299-306.

(20) Cenci, L.; Anesi, A.; Busato, M.; Guella, G.; Bossi, A. M. J. Mol. Recognit. 2016, 29, 41-50.

(21) Bertolla, M.; Cenci, L.; Anesi, A.; Ambrosi, E.; Tagliaro, F.; Vanzetti, L.; Guella, G.; Bossi, A. M. ACS Appl. Mater. Interfaces 2017, 9, 6908-6915.

(22) Wan, W.; Han, Q.; Zhang, X.; Xie, Y.; Sun, J.; Ding, M. Chem. Commun. 2015, 51, 3541-3544.

(23) Winther, B.; Moi, P.; Nordlund, M. S.; Lunder, N.; Paus, E.; Reubsaet, J. L. E. J. Chromatogr. B: Anal. Technol. Biomed. Life Sci. 2009, 877, 1359-1365.

(24) Molina, R.; Filella, X.; Auge, J. M. Clin. Biochem. 2004, 37, 505-511.

(25) Rossetti, C.; Abdel Qader, A.; Halvorsen, T. G.; Sellergren, B. r.; Reubsaet, L. Anal. Chem. 2014, 86, 12291-12298.

(26) Rossetti, C.; Switnicka-Plak, M. A.; Grønhaug Halvorsen, T.; Cormack, P. A.G.; Sellergren, B.; Reubsaet, L. Sci. Rep. 2017, 7, 44298.

(27) Torsetnes, S. B.; Nordlund, M. S.; Paus, E.; Halvorsen, T. G.; Reubsaet, L. J. Proteome Res. 2013, 12, 412-420.

(28) Winther, B.; Moi, P.; Paus, E.; Reubsaet, J. L. E. J. Sep. Sci. 2007, 30, 2638-2646.

(29) Vasapollo, G.; Sole, R. D.; Mergola, L.; Lazzoi, M. R.; Scardino, A.; Scorrano, S.; Mele, G. Int. J. Mol. Sci. 2011, 12, 5908-5945.

(30) Rossetti, C. Ph.D. Thesis, Molecularly Imprinted Polymers as new tool in proteomics: the case study of SCLC diagnosis. University of Oslo: Oslo, 2017.

(31) Qian, W.J.; Kaleta, D. T.; Petritis, B. O.; Jiang, H.; Liu, T.; Zhang, X.; Mottaz, H. M.; Varnum, S. M.; Camp, D. G., 2nd; Huang, L.; Fang, X.; Zhang, W.-W.; Smith, R. D. Mol. Cell. Proteomics 2008, 7, 1963-1973.

(32) Rossetti, C.; Ore, O. G.; Sellergren, B.; Halvorsen, T. G.; Reubsaet, L. J. A. Anal. Bioanal. Chem. 2017, 409, 5631-5643. 


\section{Magnetic Synthetic Receptors for Selective Clean-Up in Protein Biomarker Quantification}

Nicholas McKitterick, Frida Braathen, Magdalena A. Switnicka-Plak, Peter A. G. Cormack, Léon Reubsaet and Trine Grønhaug Halvorsen

\section{Content of Supplementary Material}

Page S-2 Figure S1 Extraction of NLLGLIEAK by mMIP A in $50 \mathrm{mM} \mathrm{ABC}$ and $100 \% \mathrm{MeCN}$ as referred to in RESULTS AND DISCUSSION in the manuscript

Figure S2 Extraction time and concentration of NLLGLIEAK by mMIP A. as referred to in RESULTS AND DISCUSSION in the manuscript

Page S-3 SUPPLEMENTARY RESULTS AND DISCUSSION as referred to in the manuscript

Page S-4 Table S1: PP conditions to yield non-magnetic core particles.

Table S2: Optical microscopy characterization of non-magnetic cores and magnetic cores.

Page S-5 Figure S3: SEM micrographs of poly(MAA-co-DVB-80) microspheres before and after magnetization procedure.

Figure S4: FT-IR spectrum of non-magnetic poly(MAA-co-DVB-80) microspheres.

Page S-6 Table S3: Conditions for the synthesis of magnetic and non-magnetic MIP and NIP microspheres.

Table S4: Optical microscopy characterization of imprinted and non-imprinted magnetic core-shell microspheres

Page S-7 Figure S5: SEM micrograph of magnetic core-shell mMIP A $\times 1560$ magnification $(\mathrm{scale}=20 \mu \mathrm{m})$

Table S5: Nitrogen sorption analysis data for imprinted and non-imprinted magnetic core-shell microspheres

Page S-8 Table S6: Feed compositions for macroreticular MIPs and their corresponding NIPs

Table S7: Effect of polymer compositions on the yield of the polymer products

Page S-9 Table S8: Optical microscopy characterization of magnetic MIP and NIP microspheres

Page S-10 Figure S6: SEM micrographs of MIP C and mMIP C 


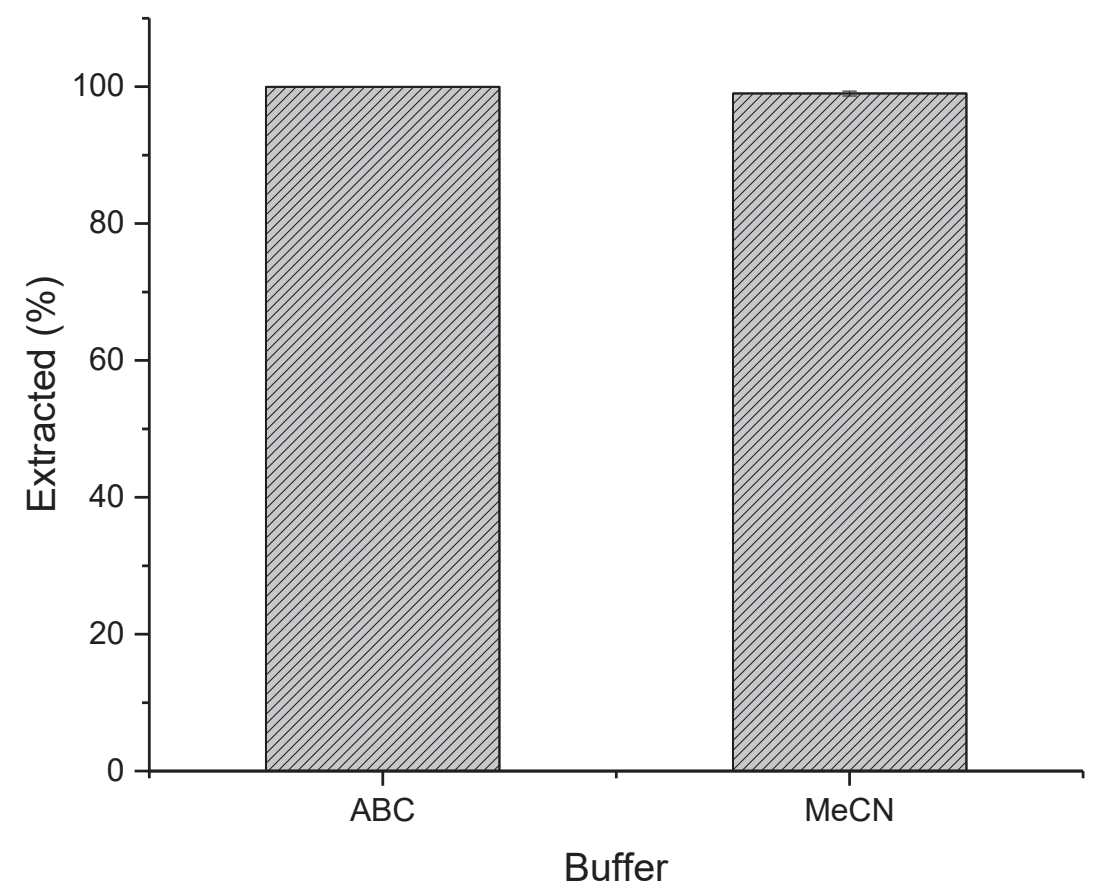

Figure S1: Extraction of NLLGLIEAK by mMIP A in $50 \mathrm{mM}$ ABC and $100 \%$ MeCN (n=3)

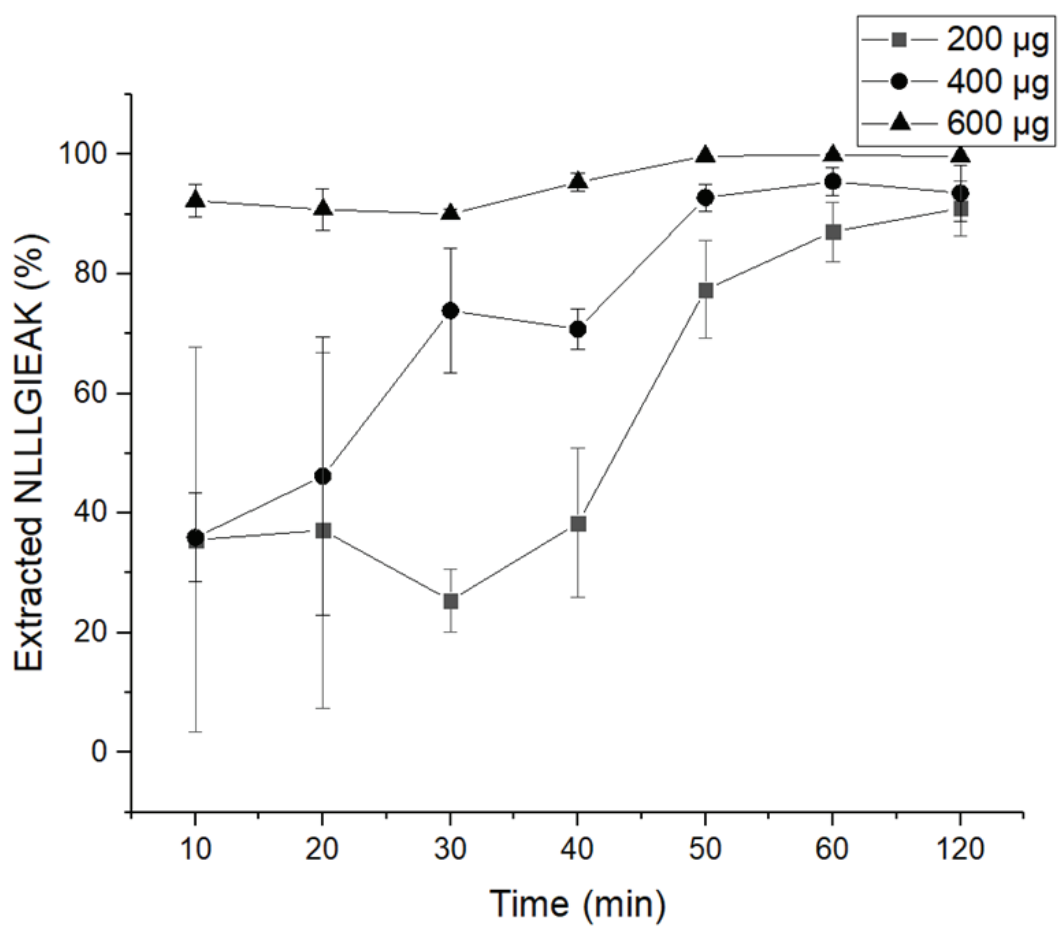

Figure S2: Extraction time and concentration of NLLGLIEAK by mMIP A. 


\section{SUPPLEMENTARY RESULTS AND DISCUSSION}

\section{mMIP A AND mMIP B AND THEIR CORRESPONDING NIPs}

Magnetic core-shell MIP microspheres (mMIP A and mMIP B) were synthesized by a two-step precipitation polymerization (PP). Firstly, porous poly(MAA-co-DVB-80) microspheres were prepared, then the magnetic component $\left(\mathrm{Fe}_{3} \mathrm{O}_{4}\right)$ incorporated into the microspheres by in-filling of the pores with a $\mathrm{Fe}_{3} \mathrm{O}_{4}$ precursor followed by a chemical treatment. The magnetised poly(MAA-co-DVB-80) microspheres were then used as seed particles in a second PP, in the presence of template, to yield magnetic core-shell MIP microspheres.

\section{Magnetization procedure}

A new procedure for the conversion of non-magnetic MIPs and NIPs into their magnetic variants was developed. The magnetization procedure begins with neutralization of the carboxylic acid groups present in the polymer using aqueous ammonium hydroxide. The carboxylate groups formed are accessible to, and show affinity for, ferrous and ferric ions, and the polymers were contacted with these ions in an $\mathrm{Fe}^{3+}$ to $\mathrm{Fe}^{2+}$ ratio of 1:75. This ratio was the same ratio used for the synthesis of magnetic microgels prepared via stop-flow lithography as reported by Hatton et al $l^{l}$. The co-precipitation of $\mathrm{FeCl}_{3}$ and $\mathrm{FeCl}_{2}$ under basic conditions results in the formation of either magnetite, $\mathrm{Fe}_{3} \mathrm{O}_{4}$, or maghemite, $\gamma$ $\mathrm{Fe}_{2} \mathrm{O}_{3} \cdot \mathrm{Fe}^{3+}$ ions have stronger affinity to carboxylate groups than $\mathrm{Fe}^{2+}$ ions, and magnetite formation is favored over magnetite formation where there are carboxylic acid/carboxylate groups in the polymers. ${ }^{1}$

${ }_{2}^{2}$ In the present work, the desired product from the co-precipitation of $\mathrm{FeCl}_{3}$ and $\mathrm{FeCl}_{2}$ was magnetite, thus the reaction was required to be carried out under anaerobic conditions. For the magnetization process, ions of $\mathrm{Fe}^{3+}$ diffuse to the carboxylate groups and are chelated, followed by the co-precipitation of ferrous and ferric ions and the formation of magnetite throughout the surfaces of the microspheres. During initial experiments (results not reported here), it was observed that insufficient removal of oxygen led to the formation of maghemite (when the solutions in which the polymer particles were incubated changed color to red, this indicated the formation of maghemite and an unsuccessful magnetization procedure where the products did not show magnetic properties). When the solutions in which the polymer particles were incubated changed color to dark brown, this was indicative of magnetite formation and successful magnetization. The formation of the magnetite phase is affected by temperature. Higher temperatures allow for the formation of a greater amount of the magnetite phase. ${ }^{3}$ For this reason, the polymers were incubated with the iron chloride solutions at $60{ }^{\circ} \mathrm{C}$ to favour magnetite formation. 


\section{Synthesis and characterization of magnetic poly(maa-co-dvb-80) microspheres}

The copolymerisation of DVB-80 and MAA under appropriate PP conditions yields the non-magnetic poly(MAA-co-DVB-80) microspheres, and the PP conditions used were similar to those reported previously ${ }^{4}$. The monomer concentration was $3.28 \% \mathrm{w} / \mathrm{v}$ (with respect to the solvent), the initiator concentration was $3.35 \mathrm{~mol} \%$ (with respect to the total number of polymerisable double bonds), and the porogen was a mixture of acetonitrile and toluene (75:25 (v/v)). High quality poly(MAA-co-DVB-80) microspheres with mean diameters around $5 \mu \mathrm{m}$ were prepared in good yield (Table S1; Figure S3).

The entire mass of non-magnetic core particles was subjected to the magnetisation procedure. The polymer incubated in the magnetization solution changed color to dark brown, indicative of the successful formation of magnetite. After the magnetization process, $80 \%$ of the mass of polymer subjected to the magnetization procedure was recovered and subjected to magnetic separation to remove any non-magnetic polymer microspheres. The fraction of magnetic particles isolated constituted $51 \%$ of the total material recovered. Incorporation of magnetite into the polymer was evaluated via the application of a magnetic field in the vicinity of the polymer particles. Migration of the polymer particles to and along a magnet was observed when a permanent magnet was applied to the base of a Petri dish containing the polymer, demonstrating that magnetite was incorporated into the polymers. The colour of the polymers was brown, which also supports magnetite formation. Optical microscopy and SEM characterization showed that the magnetization procedure did not change the size or shape of the polymer microspheres in any discernable way (Table S2; Figure S3).

Table S1: Precipitation polymerization condition to yield non-magnetic core particles.

\begin{tabular}{cccccccc}
\hline Polymer & $\begin{array}{c}\text { Components } \\
(\mathrm{mmol})\end{array}$ & $\begin{array}{c}\text { Monomer } \\
\text { conc. } \\
(\% \mathrm{w} / \mathrm{v})\end{array}$ & $\begin{array}{c}\text { AIBN } \\
\text { conc. } \\
(\mathrm{mol} \%)\end{array}$ & Solvent $(\mathrm{v} / \mathrm{v})$ & $\begin{array}{c}\text { Incubation } \\
\text { time }(\mathrm{h})\end{array}$ & $\begin{array}{c}\text { Scale } \\
(\mathrm{g})\end{array}$ & $\begin{array}{c}\text { Yield } \\
(\%)\end{array}$ \\
\hline $\begin{array}{c}\text { Non- } \\
\text { magnetic } \\
\text { cores }\end{array}$ & $\begin{array}{c}\text { DVB-80 (67.16) } \\
\text { MAA (14.29) }\end{array}$ & 3.28 & 3.35 & $\begin{array}{c}\text { ACN/toluene } \\
(75: 25)\end{array}$ & 72 & 10 & 68 \\
\hline
\end{tabular}

Table S2: Optical microscopy characterization of non-magnetic cores and magnetic cores

\begin{tabular}{cccc}
\hline \multirow{2}{*}{ Polymer } & \multicolumn{3}{c}{ Optical microscopy observation } \\
\cline { 2 - 4 } & Dispersity $^{\mathrm{a}}$ & Bead size $(\mu \mathrm{m})$ & Aggregation \\
\hline Non-magnetic cores & Polydisperse & $4-5$ & none \\
Magnetic cores & Polydisperse & $4-5$ & none \\
\hline
\end{tabular}

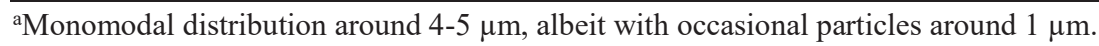



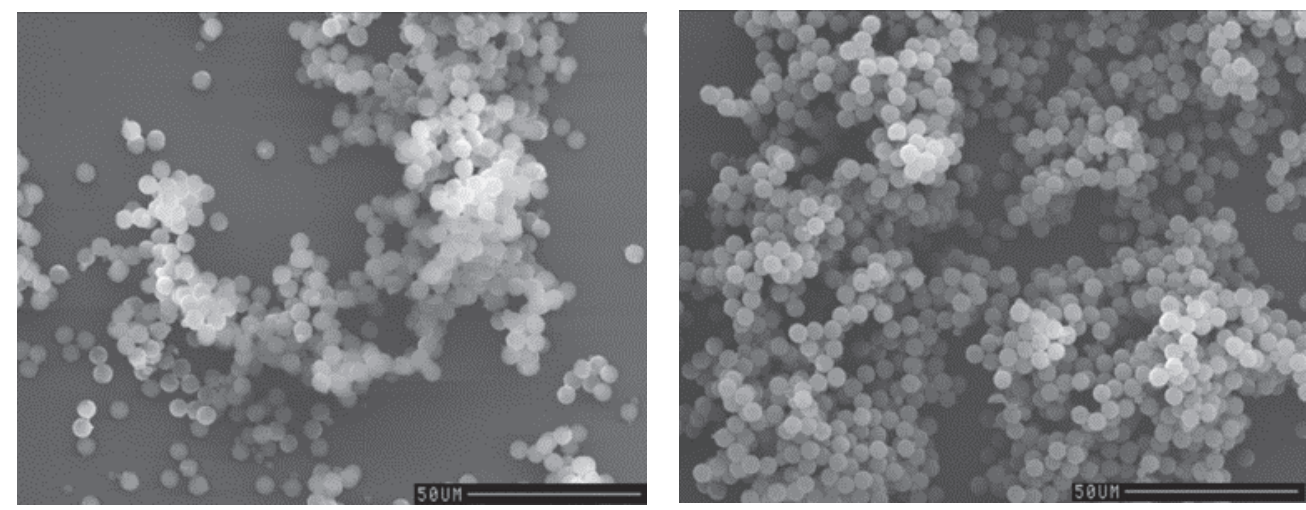

Figure S3 SEM micrographs of poly(MAA-co-DVB-80) microspheres before (left) and after (right) magnetization procedure; $\times 604$ magnification $($ scale $=50 \mu \mathrm{m})$.

An FT-IR spectrum of the poly(MAA-co-DVB-80) microspheres before magnetization is presented in Figure S4. The following characteristic peaks were observed: aliphatic C-H stretches at $2922 \mathrm{~cm}^{-1}$, carboxylic acid $\mathrm{C}=\mathrm{O}$ stretch at $1701 \mathrm{~cm}^{-1}$, aromatic $\mathrm{C}=\mathrm{C}$ stretch at $1604 \mathrm{~cm}^{-1}$, alkene stretches at 992 $\mathrm{cm}^{-1}$ and $904 \mathrm{~cm}^{-1}$, and three stretches at $832 \mathrm{~cm}^{-1}, 796 \mathrm{~cm}^{-1}$ and $711 \mathrm{~cm}^{-1}$, corresponding to para- and meta-disubstituted aromatic rings. The FT-IR analysis shows that MAA was copolymerised successfully with DVB-80.

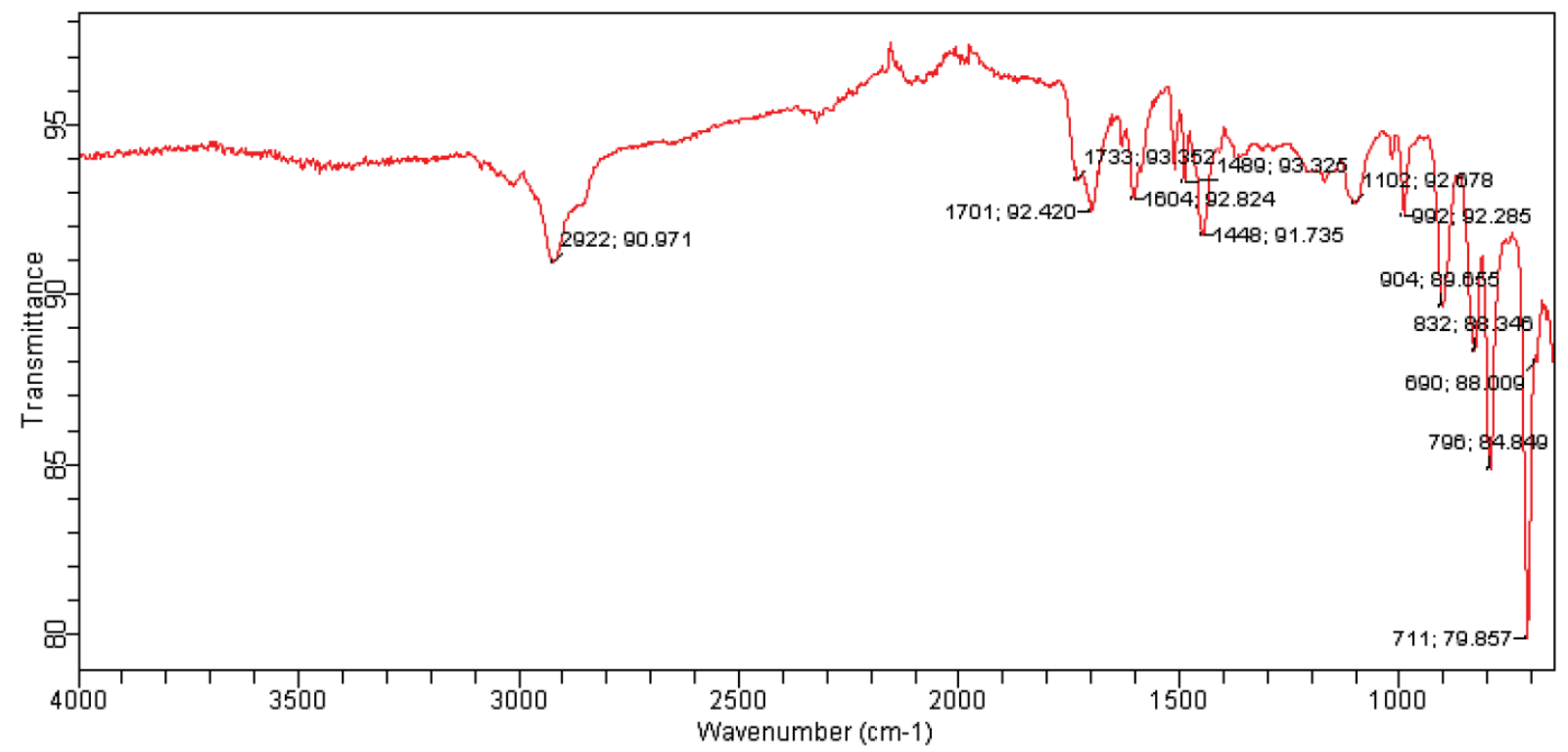

Figure S4. FT-IR spectrum of non-magnetic poly(MAA-co-DVB-80) microspheres.

\section{Synthesis and characterization of magnetic core-shell MIP microspheres and their corresponding} NIPs

Magnetic poly(MAA-co-DVB-80) microspheres were used as seed (core) particles in a second PP to install both imprinted and non-imprinted shells. A 2:1 w/w ratio of magnetic core particles to monomer 
was used for the synthesis of the core-shell microspheres. Such a ratio allowed for the synthesis of coreshell microspheres with a shell thickness of $\sim 0.1 \mu \mathrm{m}$. Non-magnetic shells grown on the surfaces of the magnetic cores were prepared with monomer and initiator concentrations at $4 \% \mathrm{w} / \mathrm{v}$ (with respect to the solvent) and $2 \mathrm{~mol} \%$ (with respect to the total number of polymerisable double bonds), respectively. 24 $\mathrm{h}$ was the polymerisation time used for the synthesis of the magnetic core-shell particles.

MIPs (mMIP A and mMIP B) and the corresponding NIPs (mNIP A and mNIP B) were prepared with the delayed addition of template (for imprinted shells) and functional monomer(s), timed $1.5 \mathrm{~h}$ after the polymerisations had started. The apparent conversions of monomer to polymer were low $(\sim 10 \%$, which equates to a mass increase of the cores of $\sim 5 \%$ ) but this is consistent with the desire to install thin shells (Table S3). Microscopy characterization revealed that good quality particles were prepared (Table S4; Figure S5)

Table S3: Conditions for the synthesis of magnetic and non-magnetic MIP and NIP microspheres.

\begin{tabular}{cccc}
\hline Polymer & $\begin{array}{c}\text { Shell description } \\
(2: 1 \text { core:shell })\end{array}$ & Template & $\begin{array}{c}\text { Yield (shell) (before } \\
\text { and after magnetic } \\
\text { separation) }(\%)\end{array}$ \\
\hline mMIP A & $\begin{array}{c}99.4 \% \text { DVB-80, 0.5\% EAMA.HCI, } \\
0.1 \% \text { BTPV }\end{array}$ & Z-NLLGLIEA[Nle] & $12 ; 0$ \\
\hline mNIP A & $99.4 \%$ DVB-80, 0.5\% EAMA.HCI, \\
$0.1 \%$ BTPV & - & $3 ; 0$ \\
mMIP B & $99.4 \%$ DVB-80, 0.5\% EAMA.HCI & Z-NLLGLIEA[Nle] & $12 ; 0$ \\
\hline mNIP B & $99.4 \%$ DVB-80, 0.5\% EAMA.HCI & - & $15 ; 2$ \\
\hline
\end{tabular}

Yield reported is the apparent yield of thin shell layer, and is subject to significant error due to the low scale of operation and loss of material during product work-up.

Table S4. Optical microscopy characterization of imprinted and non-imprinted magnetic core-shell microspheres. ${ }^{\text {a }}$

\begin{tabular}{cccc}
\hline & Dispersity & Bead size $(\mu \mathrm{m})$ & Aggregation \\
\hline mMIP A & Polydisperse & $4-5$ & none \\
mNIP A & Polydsiperse & $4-5$ & none \\
mMIP B & Polydsiperse & $4-5$ & none \\
mNIP B & Polydisperse & $4-5$ & none \\
\hline${ }^{a}$ Monomodal distribution around 4-5 $\mu \mathrm{m}$, albeit with occasional particles around $1 \mu \mathrm{m}$.
\end{tabular}




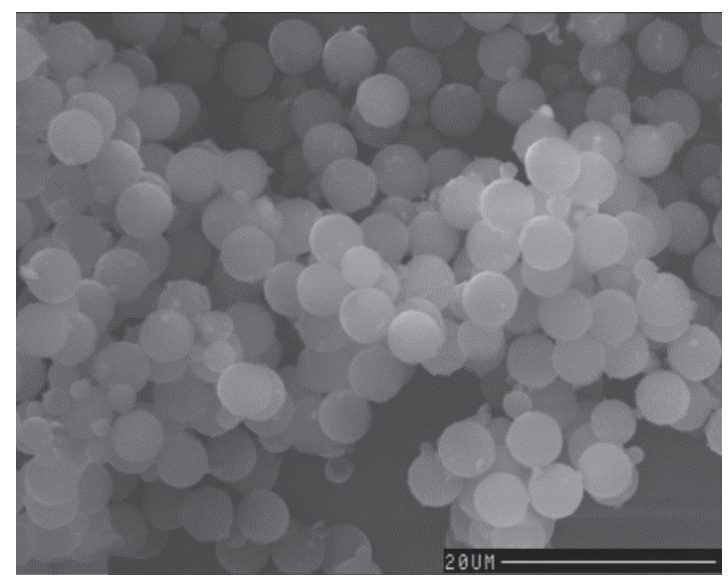

Figure S5. SEM micrograph of magnetic core-shell mMIP A $\times 1560$ magnification (scale $=20 \mu \mathrm{m}$ ).

The nitrogen sorption analysis data (Table S5) shows that the MIPs and NIPs were macroreticular; this was important to establish in view of the need for the analyte to access molecularly imprinted binding sites during mSPE. The NIPs were similar to the MIPs with respect to their porous morphologies.

Table S5. Nitrogen sorption analysis data for imprinted and non-imprinted magnetic core-shell microspheres.

\begin{tabular}{|c|c|c|c|c|c|}
\hline \multirow{2}{*}{ Polymer } & \multirow{2}{*}{$\begin{array}{l}\text { BET C } \\
\text { value }\end{array}$} & \multicolumn{2}{|c|}{$\begin{array}{l}\text { Specific surface area } \\
\left(\mathrm{m}^{2} / \mathrm{g}\right)\end{array}$} & \multirow{2}{*}{$\begin{array}{l}\text { Specific pore volume } \\
\qquad\left(\mathrm{cm}^{3} / \mathrm{g}\right)\end{array}$} & \multirow{2}{*}{$\begin{array}{l}\text { Average pore } \\
\text { diameter }(\mathrm{nm})\end{array}$} \\
\hline & & BET & Langmuir & & \\
\hline mMIP A & -137 & 468 & 670 & 0.27 & 2.96 \\
\hline mNIP A & -152 & 472 & 702 & 0.27 & 3.03 \\
\hline mMIP B & -267 & 369 & 549 & 0.21 & 3.03 \\
\hline mNIP B & -266 & 348 & 519 & 0.20 & 3.11 \\
\hline
\end{tabular}

mMIP C AND mMIP D AND THEIR CORRESPONDING NIPS

\section{Synthesis and characterization of magnetic MIP and NIP polymer microspheres}

mMIP C and mMIP D, and their corresponding NIPs, were prepared by magnetization of imprinted and non-imprinted porous polymer microspheres which had been produced via a PP protocol. Therefore, the first step was the synthesis of porous MIP microspheres (and their corresponding NIPs) with ZNLLGLIEA[Nle] as template, which was followed by the magnetization procedure. The MIPs and NIPs 
were synthesised according to a PP method described elsewhere ${ }^{5}$. The feed compositions for the MIPs and NIPs are shown in Table S6. Two pairs of polymers were synthesized: two MIPs (MIP C and MIP D) and two NIPs (NIP C and NIP D). The polymers were produced on a $1 \mathrm{~g}$ monomer scale, with monomer and initiator concentrations of $2 \% \mathrm{w} / \mathrm{v}$ (with respect to the solvent) and $2 \mathrm{~mol} \%$ (with respect to the total number of moles of polymerisable double bonds), respectively. $24 \mathrm{~h}$ was the polymerisation time. The polymers were produced in good yields (Table S7).

Table S6. Feed compositions for macroreticular MIPs and their corresponding NIPs.

\begin{tabular}{|c|c|c|c|c|c|c|}
\hline Polymer & Template (mmol) & $\begin{array}{c}\text { Functional } \\
\text { Monomer (mmol) }\end{array}$ & $\begin{array}{c}\text { DVB-80 } \\
(\mathrm{mmol})\end{array}$ & $\begin{array}{l}\text { Solvent } \\
(\mathrm{mL})\end{array}$ & $\begin{array}{l}\text { AIBN } \\
(\mathrm{mol} \%)\end{array}$ & $\begin{array}{c}\text { PMP or/and } \\
\text { TBA.OH (mmol) }\end{array}$ \\
\hline MIP C & $\begin{array}{c}\text { Z-NLLGLIEA[Nle] } \\
(0.028)\end{array}$ & EAMA.HCI (0.28) & 14.92 & $\begin{array}{l}\mathrm{ACN}(48) \\
\mathrm{DMSO}(2)\end{array}$ & 2 & PMP (0.024) \\
\hline NIP C & - & EAMA.HCI (0.28) & 14.92 & $\begin{array}{l}\mathrm{ACN}(48) \\
\operatorname{DMSO}(2)\end{array}$ & 2 & PMP (0.024) \\
\hline MIP D & $\begin{array}{c}\text { Z-NLLGLIEA[Nle] } \\
(0.028)\end{array}$ & $\begin{array}{c}\text { EAMA.HCI }(0.28) \\
\text { BTPV }(0.04)\end{array}$ & 14.92 & $\begin{array}{l}\mathrm{ACN}(48) \\
\mathrm{DMSO}(2)\end{array}$ & 2 & $\begin{array}{l}\text { TBA.OH (0.04), } \\
\text { PMP }(0.024)\end{array}$ \\
\hline NIP D & - & $\begin{array}{c}\text { EAMA.HCI }(0.28) \\
\text { BTPV }(0.04)\end{array}$ & 14.92 & $\begin{array}{l}\mathrm{ACN}(48) \\
\operatorname{DMSO}(2)\end{array}$ & 2 & $\begin{array}{l}\text { TBA.OH (0.04), } \\
\text { PMP }(0.024)\end{array}$ \\
\hline
\end{tabular}

Table S7. Effect of polymer compositions on the yield of the polymer products.

\begin{tabular}{cccc}
\hline Polymer & Polymer name & Template & $\begin{array}{c}\text { Yield } \\
(\%)\end{array}$ \\
\hline MIP C & poly(EAMA.HCl-co-DVB-80) & Z-NLLGLIEA[Nle] & 56 \\
NIP C & poly(EAMA.HCl-co-DVB-80) & - & 55 \\
MIP D & poly(BTPV-co-EAMA.HCl-co-DVB-80) & Z-NLLGLIEA[Nle] & 57 \\
NIP D & poly(BTPV-co-EAMA.HCl-co-DVB-80) & - & 53 \\
\hline
\end{tabular}

The entire mass of each polymer was subjected to the magnetization procedure. Two cycles of the magnetization procedure were sufficient in order to convert the polymers into magnetic variants, and it should be noted that the magnetization procedure can be applied to porous polymers that do not contain 
carboxylic acid groups in their structure. The polymers incubated in the magnetization solution changed color to dark brown, which indicated the presence of magnetite within the porous polymer microspheres. Magnetic separations were performed to separate the magnetic polymer particles from any nonmagnetized particles. Firstly, dried products were transferred into clean beakers and washed with EtOH $(\sim 50 \mathrm{~mL})$. The products dispersed in EtOH were mixed with a glass rod. Thereafter, a permanent magnet was held against the base of the beakers, and the beakers with dispersed products left for $15 \mathrm{~s}$. At this stage, the supernatants were collected. The whole procedure was repeated at least four times until the supernatants became clear. The magnetic particles were isolated by vacuum filtration using a Vacuumbrand Vacuum System (2.0 mbar) on $0.45 \mu \mathrm{m}$ nylon membrane filters. The isolated products were transferred again into pre-weighed vials and dried overnight in a Townson \& Mercer vacuum oven (60 mbar) at $70{ }^{\circ} \mathrm{C}$. The yields of the magnetisation process were higher for the MIPs than for NIPs ( $2 \%$, $28 \%, 8 \%$ and $22 \%$ for mNIP C, mMIP C, mNIP D, and mMIP D, respectively).

SEM micrographs of the non-magnetic and magnetic MIPs and NIPs (example of MIP is shown in Figure S6) revealed that discrete particles in the micron-sized range had been produced, although the microspheres were polydisperse (possibly because of the presence of DMSO as a co-solvent). Optical microscopy and SEM analysis showed that the magnetization procedure did not change the size or shape of the polymer microspheres in any discernable way (Table S8; Figure S6).

Table S8. Optical microscopy characterization of magnetic MIP and NIP microspheres.

\begin{tabular}{cccc}
\hline \multirow{2}{*}{ Polymer } & \multicolumn{3}{c}{ Optical microscopy observation } \\
\cline { 2 - 4 } & Dispersity & Bead size $(\mu \mathrm{m})$ & Aggregation \\
\hline mNIP C & Polydisperse & $4-5$ & Chemical \\
mMIP C & Polydisperse & $1-5$ & Chemical \\
mNIP D & Polydisperse & approx. 1 & Chemical \\
mMIP D & Polydisperse & approx. 1 & Chemical \\
\hline
\end{tabular}



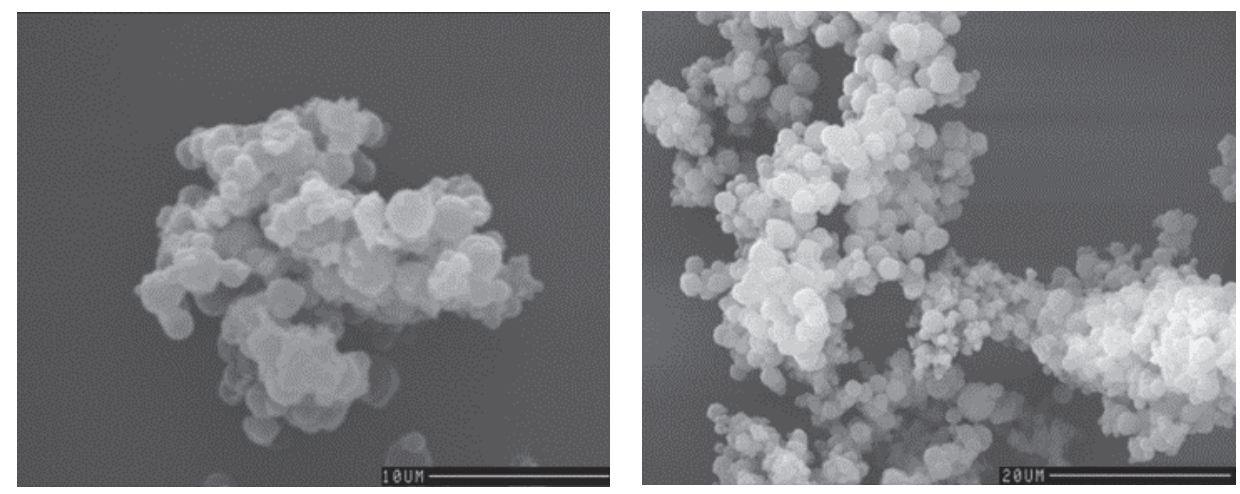

Figure S6. SEM micrographs of MIP C (left) and mMIP C (right); magnifications: $\times 1680$ and $\times 3380$ (scales $=10 \mu \mathrm{m}$ and $20 \mu \mathrm{m}$ for left and right SEM images, respectively).

\section{REFERENCES}

1. Suh, S. K.; Yuet, K.; Hwang, D. K.; Bong, K. W.; Doyle, P. S.; Hatton, T. A., Synthesis of nonspherical superparamagnetic particles: in situ coprecipitation of magnetic nanoparticles in microgels prepared by stop-flow lithography. JACS 2012, 134, 7337-7343.

2. Huang, J.; Wan, S.; Guo, M.; Yan, H., Preparation of narrow or mono-disperse crosslinked poly ((meth) acrylic acid)/iron oxide magnetic microspheres. J. Mater. Chem. 2006, 16 (46), 45354541.

3. Lavrynenko, S. N.; Chebanov, V. A.; Sofronov, D. S., Efficient extraction of radioactive isotopes of cobalt, europium, cerium, strontium, and copper from aqueous solutions. Int. J. Environ. Sustain. 2016, 5, DOI: 10.24102/ijes.v5i1.666.

4. Beltran, A.; Marcé, R. M.; Cormack, P. A.; Sherrington, D. C.; Borrull, F., Selective solidphase extraction of amoxicillin and cephalexin from urine samples using a molecularly imprinted polymer. J. Sep. Sci. 2008, 31, 2868-2874.

5. Rossetti, C.; Świtnicka-Plak, M. A.; Halvorsen, T. G.; Cormack, P. A.; Sellergren, B.; Reubsaet, L., Automated Protein Biomarker Analysis: on-line extraction of clinical samples by Molecularly Imprinted Polymers. Sci. Rep. 2017, 7, 44298. 
II 



\title{
Facilitating serum determination of neuron specific enolase at clinically relevant levels by coupling on-line molecularly imprinted solid-phase extraction to LC-MS/MS
}

\author{
Nicholas McKitterick ${ }^{\mathrm{a}}$, Tugrul Cem Bicak ${ }^{\mathrm{b}}$, Peter A.G. Cormack ${ }^{\mathrm{b}}{ }^{* *}$, Léon Reubsaet ${ }^{\mathrm{a}}$, \\ Trine Grønhaug Halvorsen ${ }^{\text {a, * }}$
}

a Section for Pharmaceutical Chemistry, Department of Pharmacy, University of Oslo, PO Box 1068, Blindern, 0316, Oslo, Norway

${ }^{\mathrm{b}}$ WestCHEM, Department of Pure and Applied Chemistry, University of Strathclyde, Thomas Graham Building, 295 Cathedral Street, Glasgow, G1 1XL, Scotland, UK

\section{H I G H L I G H T S}

- Neuron specific enolase (NSE) is a low abundance small cell lung cancer biomarker.

- Proteotypic peptide analogue used as template for molecularly imprinted polymers.

- Column-packed polymers employed for on-line extraction prior to LC-MS/ MS.

- Target peptide capture from tryptic digested human serum demonstrated.

- Achieved LOD and LOQ is below reference level in healthy humans.

\section{A R T I C L E I N F O}

\section{Article history:}

Received 17 September 2020

Accepted 12 October 2020

Available online 15 October 2020

\section{Keywords:}

On-line solid-phase extraction

Molecularly imprinted polymers

Liquid chromatography tandem mass

spectrometry

Low-abundant biomarkers

Bottom-up proteomics
G R A P H I C A L A B S T R A C T

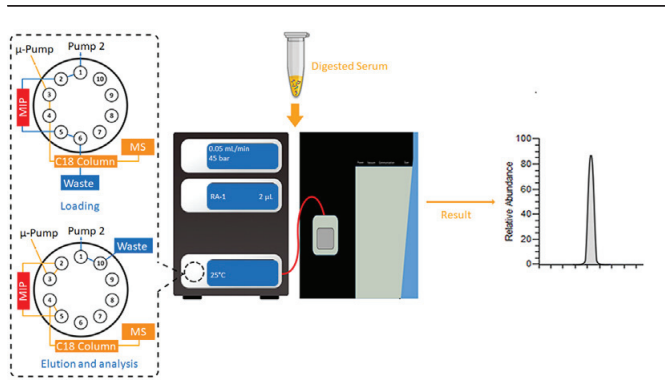


untreated digested human serum were $100.8 \pm 6.2 \%$ and $61.6 \pm 1.9 \%$, respectively. Extractions of ELPLYR from spiked untreated digested serum were linear in the range of $7.5-375 \mathrm{ng} \mathrm{mL} \mathrm{m}^{-1}\left(\mathrm{R}^{2}=0.99\right)$. The limit of detection (LOD) and limit of quantification (LOQ) for the biomarker in digested serum were estimated to be $1.8 \mathrm{ng} \mathrm{mL}-1$ and $6.0 \mathrm{ng} \mathrm{mL} L^{-1}$, respectively, which is below the median reference level of NSE in humans $\left(8.6 \mathrm{ng} \mathrm{mL} \mathrm{m}^{-1}\right)$. This work sets in place the basis for a new diagnostic tool for SCLC that is sensitive, robust, automated, and antibody-free, and which works very well with complex human plasma samples.

\section{Introduction}

The identification and quantification of biomarkers of disease is essential for the diagnosis, treatment, and long-term monitoring of many common health conditions, including cancers. In this regard, several thousand putative biomarkers have been reported in the literature to date, and many new biomarkers are discovered every single year [1]. The classical methods used for biomarker analysis are normally antibody-based methods, such as enzyme-linked immunosorbent assays (ELISAs) [2]. Whilst such antibody-based methods are usually sensitive and rapid, the raising of antibodies against biomarkers can be an expensive undertaking. Furthermore, the lead-in times for the development and production of new antibodies tend to be lengthy, which means that new antibody production struggles to keep pace with the rapid rate of discovery of new biomarkers. Furthermore, antibody-based assays can give rise to false positive and false negative results, although this particular limitation can be addressed to some extent by combining antibody clean-up with mass spectrometric determination. Nevertheless, the development and exploitation of antibody-free strategies in combination with mass spectrometry for targeted, rapid, sensitive, and accurate biomarker detection is very timely and of critical importance to human health.

Molecularly imprinted polymers (MIPs) are synthetic polymers with unique physicochemical properties that allow the targeted capture of neutral or charged molecules [3]. When used as antibody binding mimics, MIPs can bind strongly and selectively to a range of targets, from small molecule analytes through to macromolecules such as proteins, and even to whole cells [4]. Most commonly, the affinity of a MIP for its target is based upon non-covalent intermolecular interactions, such as hydrogen bonding, however the size and shape of the binding sites within the MIP play a role too [5].

MIPs have been shown to be robust, reusable materials [6], and, compared to the production of typical single-use antibodies, they can be produced efficiently using streamlined synthetic protocols. This is a major reason why MIPs are attracting interest as costeffective alternatives to antibodies for the analysis of some biomarkers. These recent developments are in addition to the use of MIPs in a range of other fields, including electrochemical sensors [7], drug delivery [8], protein crystallization [9], catalysis [10], and in separation science (e.g., solid-phase extraction, SPE) [11]. MIPs have been used extensively for numerous applications in separation science for over 20 years [11]. Once optimised for loading and elution solvents, the versatility of MIPs allows for successful off-line and on-line SPE for a range of targets, including biomolecules [6]. On-line molecularly imprinted solid-phase extraction (MISPE) includes the field of imprinted materials incorporated into appropriate physical formats for packing into columns for on-line chromatographic analysis [12]. On-line MISPE in combination with liquid chromatography-tandem mass spectrometry (LC-MS/MS) offers a robust automated platform for the analysis of biomarkers, and MISPE columns can be interfaced readily with both micro- and
nano-LC systems [13-15]. A key advantage of moving to an automated, on-line mode of operation is reduced manual handling, which gives higher throughput and better reproducibility and repeatability than the analogous off-line methods.

A key development of MIPs is the extraction of biomolecules from biological fluids (e.g., human serum). MIPs targeting many proteins and peptides have been developed. For proteins, the imprinting strategy involves either whole protein imprinting or imprinting of a smaller fraction of the protein such as the epitope or tryptic signature peptide [16]. Whole protein imprinting has been utilised for some high abundant proteins, while imprinting of a smaller fraction of the protein is used more commonly for lowabundant proteins [16]. Typically, the MIPs are exploited as microparticles and can be packed into SPE columns used in combination with LC-MS/MS for the selective extraction and quantification of low abundant protein and peptide biomarkers in complex matrices (e.g., human serum) [17]. MIPs targeting the signature peptide of the small cell lung cancer (SCLC) biomarker progastrin-releasing peptide (ProGRP) were developed for use in on-line analysis [12] and used to extract the nonapeptide NLLGLIEAK from human serum, including from serum samples from patients diagnosed with SCLC. ProGRP is the most selective biomarker known for the detection and follow-up of SCLC (i.e., most patients testing negative are not in a diseased state); however, in the clinic, ProGRP is usually determined in parallel with a second SCLC biomarker, neuron-specific enolase (NSE). NSE is present in elevated levels in a range of cancers (e.g., neuroblastoma, melanomas, and breast cancers) [18], however the combined determination of NSE and ProGRP improves the accuracy of the SCLC diagnosis. MIPs targeting NSE have been developed for use in electrochemical sensors [19], but not for off-line or on-line MISPE work. A method determining NSE from serum using LC-MS/MS and the bottom-up approach after immunocapture of the intact protein has been developed previously [18]. In this work, the peptide ELPLYR was identified as a suitable signature peptide owing to its specificity combined with strong ionisation in electrospray ionisation. Therefore, ELPLYR presents an attractive template for MIPs for use in NSE determination as well.

The objective of the present work was to develop MIPs in an appropriate physical format for use as molecularly selective sorbents in on-line MISPE, for the capture of the signature peptide of NSE (the hexapeptide ELPLYR) from biofluids. To this end, precipitation polymerization was used to deliver high quality, molecularly imprinted polymer microspheres that could be packed directly into trap columns. Subsequently, an on-line MISPE methodology was devised and optimised with a view to extracting ELPLYR from complex matrices, thereby validating the potential of MIPs for the analysis of NSE in human serum. Success will represent a significant advancement to the state-of-the-art and diagnostic power since it will now be possible to determine two diagnostically important SCLC biomarkers in human serum by MIP-based enrichment of signature peptides followed by LC-MS/MS. 


\section{Materials and methods}

\subsection{Chemicals and Reagents}

The peptide template (Z-ELPLY[Nle], 98.51\%) was purchased from LifeTein (Somerset, NJ, USA). 1,2,2,6,6-Pentamethylpiperidine (PMP, 99\%), tetrabutylammonium hydroxide solution (TBA.OH, $1.0 \mathrm{M}$ in methanol, $25 \%$ ), acetonitrile (ACN, $99 \%$ ), divinylbenzene-80 (DVB-80, 80\% DVB isomers and 20\% ethylvinylbenzene isomers), tetrahydrofuran (THF, 99\%), methanol ( $\mathrm{MeOH}, 99.8 \%)$, dimethyl sulfoxide (DMSO, 99\%), hydrochloric acid ( $\mathrm{HCl}, 37 \%)$, formic acid (FA, MS grade, $\geq 98$ ), dithiothreitol ( $\geq 98 \%$, DTT) and iodoacetic acid ( $\geq 98 \%$, IAA) were purchased from Sigma Aldrich, St Louis, MO, USA. $N$-3,5-bis(Trifluoromethyl)-phenyl- $N^{\prime}-4$-vinylphenylurea (TPVU, purity $>95 \%$ ) was kindly donated by Malmö University. 2,2'-Azobisisobutyronitrile (AIBN, $\geq 98 \%$ ) was purchased from BDH (Dubai, UAE). Acetonitrile LC-MS grade (MeCN, 99.9\%), methanol LC-MS grade $(\mathrm{MeOH}, 99.9 \%)$, and acetic acid $(\mathrm{AcOH}, 100 \%)$ were purchased from Merck (Darmstadt, Germany). Ammonium bicarbonate (BioUltra, $>99.5 \%$ ) was purchased from Fluka (Milwaukee, WI, USA). Purified NSE ( $\gamma \gamma$-dimer, Protein ID: P09104) was obtained from Scripps Laboratories (San Diego, CA, USA). Stable isotope labelled internal standard (IS) peptide ELPLY $\left[R_{-}{ }^{13} C_{6}^{15} N_{2}\right](>95 \%)$ was purchased from Innovagen (Lund, Sweden). Bovine serum albumin (BSA) and trypsin (TPCK-treated) from bovine pancreas (sequencing grade) were purchased from Sigma Aldrich. Water was filtered through a Merck Millipore Milli-Q Integral 3 water dispenser (resistivity: $18.2 \mathrm{M} \Omega \mathrm{cm}$ ). Human serum from consenting healthy individuals was obtained from Oslo University Hospital, Ullevål (Oslo, Norway). All serum samples were stored at $-32^{\circ} \mathrm{C}$.

\subsection{Preparation of proteins, standard solutions, and purification of reagents}

NSE concentration was determined via UV absorbance $(280 \mathrm{~nm}$, A280) and the NSE stock diluted to the desired concentrations with $50 \mathrm{mM}$ ammonium bicarbonate $(\mathrm{ABC})$ and stored at $-20^{\circ} \mathrm{C}$.

A stock solution of the IS peptide was prepared in water at a concentration of $10 \mathrm{mM}$. The standards were diluted in $50 \mathrm{mM} \mathrm{ABC}$ for further use.

DVB-80 was passed through a short column of neutral alumina prior to use, and AIBN was recrystallized from acetone at low temperature.

\subsection{MIP syntheses}

See Supplementary Information for the MIP characterization data.

MIP 1 was prepared via precipitation polymerization using an adaptation of literature methods: To a borosilicate Kimax tube was added Z-ELPLY[Nle] (6.6 mg, $7.5 \mu \mathrm{mol})$, PMP $(1.8 \mu \mathrm{L}, 10 \mu \mathrm{mol})$, DVB80 (1.01 g, $1.1 \mathrm{~mL}, 7.74 \mathrm{mmol}), \mathrm{MeCN}(20 \mathrm{~mL})$ and THF (2.5 mL), and the mixture ultrasonicated for 2 min until a clear solution was obtained. TPVU $(26.2 \mathrm{mg}, 70 \mu \mathrm{mol})$ and TBA.OH $(70 \mu \mathrm{L}, 70 \mu \mathrm{mol})$ were then added, and the mixture ultrasonicated for a further 2 min to dissolve all components. Then, AIBN $(19.7 \mathrm{mg}, 120 \mu \mathrm{mol})$ was added and the mixture ultrasonicated for an additional $5 \mathrm{~min}$ and purged with oxygen-free nitrogen gas for $10 \mathrm{~min}$ at ambient temperature prior to sealing of the reaction vessel under nitrogen. The sealed tube was then placed into a pre-heated incubator (UVP hybridizer oven, Analytik Jena, Jena, Germany) at $60^{\circ} \mathrm{C}$, and left for $48 \mathrm{~h}$ at a rotation speed of $\sim 8 \mathrm{rpm}$, during which time a milky suspension of polymer microspheres formed. After cooling to room temperature, the polymer microspheres were isolated from the reaction medium by vacuum filtration on a $0.45 \mu \mathrm{m}$ nylon membrane filter and washed sequentially with $\mathrm{MeCN}(50 \mathrm{~mL})$, $\mathrm{MeOH} / 0.1 \mathrm{M}$ aq. $\mathrm{HCl}(90 / 10, \mathrm{v} / \mathrm{v}, 50 \mathrm{~mL})$ and $\mathrm{MeOH}(50 \mathrm{~mL})$. Finally, the product was dried overnight in a vacuum oven $\left(60^{\circ} \mathrm{C}, 50 \mathrm{mbar}\right)$ to constant mass (yield: 49\%). The corresponding non-imprinted polymer (NIP), NIP 1, was synthesized and isolated using the same procedure, except that the template and PMP were omitted from the synthetic protocol (Yield: $45 \%$ ).

MIP 2 was prepared similarly to MIP 1 , however higher amounts of TPVU (13.1 mg, $35 \mu \mathrm{mol})$ and TBA. OH ( $35 \mu \mathrm{L}, 35 \mu \mathrm{mol})$ were used and the yield of product was $51 \%$. The corresponding NIP, NIP 2 , was synthesized and isolated using the same procedure as MIP 2, except that the template and PMP were omitted from the synthetic protocol (Yield: 48\%).

\subsection{Column packing}

Each polymer (MIP 1 and MIP 2 and their corresponding NIPs) was wet packed into $1.5 \times 5 \mathrm{~mm}$ PEEK cartridges. For this, each polymer $(6 \mathrm{mg}$ ) was suspended in $380 \mu \mathrm{L} \mathrm{MeCN}$ prior to ultrasonication for $5 \mathrm{~min}$. The polymers were vacuum-packed using heptane as the packing solvent at 100 bar. Each packed column was inspected under an optical microscope and asymmetry analysis performed to evaluate the packing quality (see Supporting Information).

\subsection{On-line MISPE-liquid chromatography-tandem mass spectrometry}

The chromatographic system consisted of an ISO-3100A loading pump, an LPG-3400M pump with a degasser, a WPS-3000TRS autosampler, and an FLM3000 flow-manager (all Dionex, Sunnyvale, CA, USA). Extraction was performed by a MIP trap column. The chromatographic separation was carried out using an Aquasil $C_{18}$ analytical column (Thermo Scientific, $100 \AA$, $3 \mu \mathrm{m}, 50 \mathrm{~mm} \times 1 \mathrm{~mm}$ ). The extraction was performed by injection of $2 \mu \mathrm{L}$ of sample onto the MIP trap column. The MIP was then washed using an isocratic flow of 97:3 $20 \mathrm{mM} \mathrm{FA:MeCN}$ at $20 \mu \mathrm{L} \mathrm{min}^{-1}$ for $10 \mathrm{~min}$ (see set-up in Fig. 1A). Following extraction, the system was switched to forward-flush the sample through the MIP column, via the analytical column, and finally into the MS for analysis. The microflow pump was directed to waste and kept at an isocratic flow of $50 \mu \mathrm{L} \mathrm{min}{ }^{-1}$ at $100 \%$ mobile phase A (20 mM FA and MeCN 95:5, v/ v) during extraction (i.e., for the first $10 \mathrm{~min}$ ). After $10 \mathrm{~min}$ of loading, the chromatographic separation was performed using a 20 min linear-gradient from 0 to $85 \%$ mobile phase B (20 mM FA and MeCN 5:95,v/v) after 3 min isocratic elution by mobile phase $A$. After the gradient was run, the column was washed for 3 min with $90 \%$ mobile phase B and re-equilibrated with mobile phase $A$ for $20 \mathrm{~min}$. The column temperature was set and kept constant at $25^{\circ} \mathrm{C}$.

A triple quadrupole mass spectrometer (TSQ Quantum Access, Thermo Scientific, Waltham, MA, USA) was used to determine the signature peptide and its corresponding IS by selected reaction monitoring (SRM) in positive mode. The following transitions pairs were monitored: for NSE- $\gamma$ signature peptide ELPLYR, $395.7 \rightarrow 274.7$ and $395.7 \rightarrow 548.3$ with collision energy (CE) of $14 \mathrm{~V}$; for the ELPLYR IS, $401.0 \rightarrow 279.7$ and $401.0 \rightarrow 558.3$ with a CE of $14 \mathrm{~V}$. The heated capillary temperature was set at $265^{\circ} \mathrm{C}$, and the spray voltage was $4.0 \mathrm{kV}$. Auxiliary gas $\left(\mathrm{N}_{2}\right)$ : 10 arb., sheath gas $\left(\mathrm{N}_{2}\right)$ : 5 arb. TSQ data were processed by Xcalibur's QualBrowser (version 2.2 SP 1.48, Thermo Scientific), and MS responses based on the peak intensity, automatically processed by genesis peak detection algorithm, were used. Among them, only peaks with a signal-to-noise $(\mathrm{S} / \mathrm{N})$-ratio above 10 and with retention time and ion ratios corresponding to those of reference samples at high 


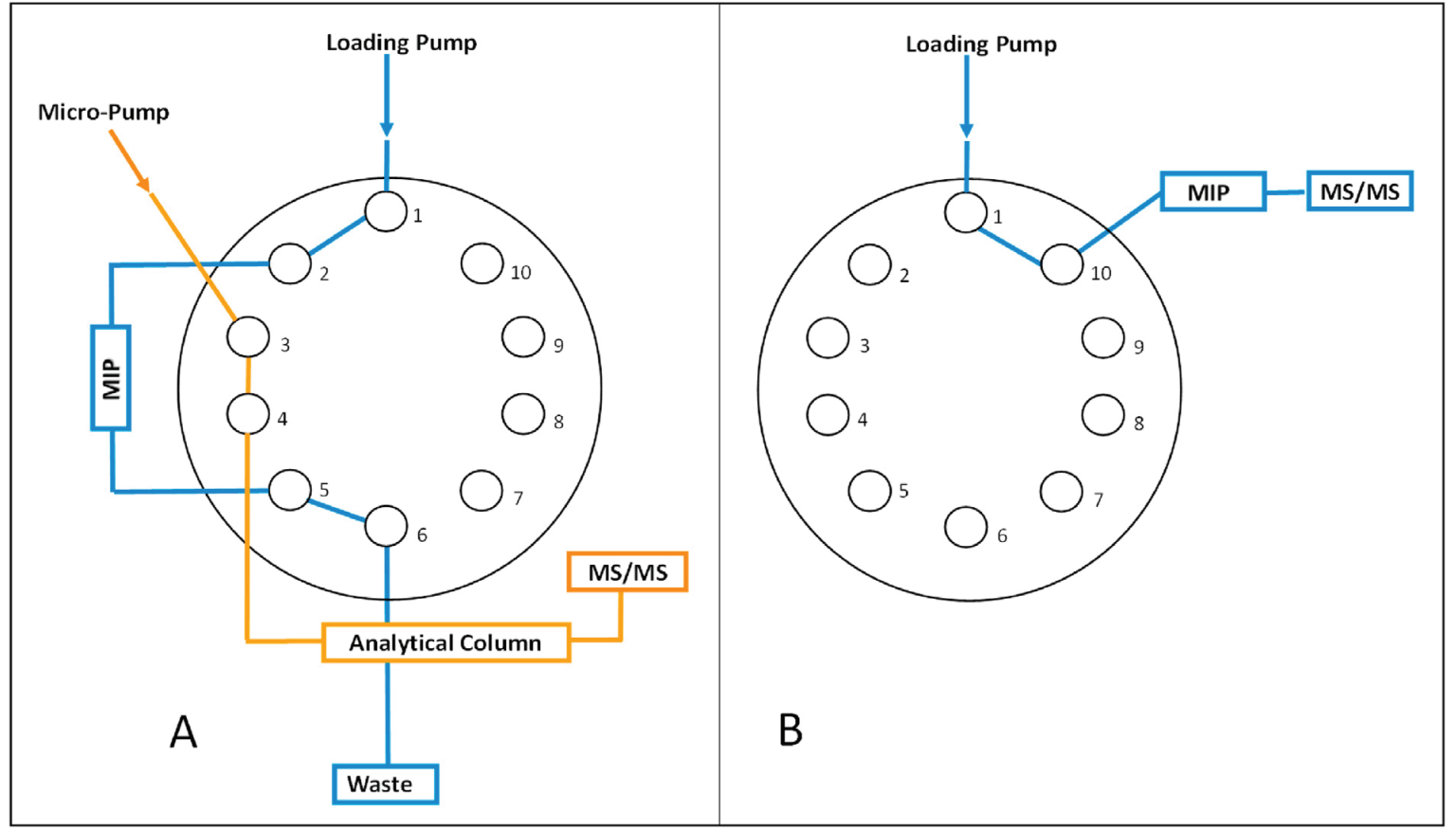

Fig. 1. (A) The on-line MISPE system. Firstly, the analyte is injected onto the MIP (left). Following sample loading, the system switches and the analyte is gradient eluted onto a $\mathrm{C}_{18}$ analytical column before determination by a triple quadrupole MS (right). (B) Set-up for initial optimisation. The MIP (or NIP) is coupled directly to the MS.

concentration were considered.

\subsection{Method optimisation}

\subsubsection{Retention factor ( $k$ ) calculation}

The retention factor, $\mathrm{k}$, was calculated using the following equation:

$\mathrm{k}=\left(t_{R}-t_{0}\right) / t_{0}$

where $t_{R}=$ Retention time, $t_{0}=$ hold-up time.

\subsubsection{Optimisation of loading mobile phase composition and flow} rate

MIP 1 and MIP 2 (and their corresponding NIPs) were coupled directly to the MS (see Figs. 1B) and Fig. $2 \mu \mathrm{L}$ of ELPLYR IS was injected directly onto the MIP/NIP columns. This LC system consisted of the ISO-3100A loading pump, the WPS-3000TRS autosampler, and the FLM3000 flow-manager. The loading mobile phases consisted of three different ratios of $20 \mathrm{mM} \mathrm{FA}$ and $\mathrm{MeCN}$ (100:0, 95:5, and 90:10 v/v). Three different flow rates were used: 20, 30, and $40 \mu \mathrm{L} \mathrm{min}{ }^{-1}$.

2.6.3. Fine-tuning of the loading mobile phase composition

The loading mobile phase was optimised further using MIP 1 and NIP 1 . As above, $2 \mu \mathrm{L}$ of ELPLYR IS was injected directly onto the columns. The loading mobile phase consisted of 100:0, 99:1, 98:2, 97:3, 96:4 and 95:5 v/v $20 \mathrm{mM}$ FA and MeCN. The flow rate was set to $20 \mu \mathrm{L} \mathrm{min}{ }^{-1}$.

\subsubsection{Optimisation of loading time (extraction time)}

The loading time was evaluated using MIP 1 as a trap column (Fig. 1A). ELPLYR IS ( $2 \mu \mathrm{L}$ ) was injected onto MIP 1 using the loading mobile phase consisting of 97:3 $20 \mathrm{mM}$ FA:MeCN at $20 \mu \mathrm{L} \mathrm{min}{ }^{-1}$. The following loading (extraction) times were evaluated: 5, 7.5, 10,
12.5 and $20 \min (\mathrm{n}=3)$. Digested BSA peptides $(50 \mathrm{nM}, 2 \mu \mathrm{L})$ were analysed at 5,10, and $20 \mathrm{~min}$ to evaluate the selectivity and optimal extraction time for sample clean-up (For BSA MS transitions see Supplementary Information Table S1).

\subsubsection{Imprinting factor (IF) calculation}

IFs were calculated for each set of conditions using the $\mathrm{k}$ values for each MIP and its respective NIP using the following equation:

$I F=\mathrm{k}(M I P) / \mathrm{k}(N I P)$<smiles>CC(C)C[C@H](NC(=O)[C@H](CCC(=O)O)NN)C(=O)N[C@@H](CC(C)C)C(=O)N[C@@H](Cc1ccc(O)cc1)C(=O)N[C@@H](CCCNC(=N)N)C(=O)O</smiles><smiles>[B]C(NC(=O)[C@H](CCC(=O)O)NC(=O)[C@H](CCC(=O)O)NC(=O)[C@H](CC(C)C)NC(=O)[C@H](CC(C)C)NC(=O)[C@H](Cc1ccc(O)cc1)NC(=O)OCc1ccccc1)C(=O)O</smiles>

Fig. 2. Chemical structures of the NSE signature peptide (ELPLYR) (A) and the structural analogue of the NSE signature peptide used as template during MIP syntheses (ELPLY[Nle]) (B). 


\subsection{Initial method evaluation}

\subsubsection{Protein digestion}

NSE- $\gamma$ standard solutions were diluted by ABC to a volume and concentration of $470 \mu \mathrm{L}$ and $53.2 \mu \mathrm{g} \mathrm{mL}^{-1}$, respectively. $10 \mu \mathrm{L}$ of $2.5 \mathrm{mM}$ DTT (freshly prepared in $50 \mathrm{mM} \mathrm{ABC}$ ) was added to the protein mixture and incubated at $60{ }^{\circ} \mathrm{C}(800 \mathrm{rpm})$ for $20 \mathrm{~min}$. Afterward, the solution was cooled, and $10 \mu \mathrm{L}$ of $10 \mathrm{mM}$ IAA (freshly prepared in $50 \mathrm{mM} \mathrm{ABC}$ ) was added. Incubation was carried out for $15 \mathrm{~min}$ at room temperature $(800 \mathrm{rpm})$ in the dark. Digestion was initiated by the addition of trypsin $\left(10 \mu \mathrm{L}, 125 \mu \mathrm{g} \mathrm{mL} \mathrm{m}^{-1}\right.$ in $50 \mathrm{mM} \mathrm{ABC}$ ) for an enzyme to substrate ratio of 1:20 (w/w) overnight at $37{ }^{\circ} \mathrm{C}$. The resulting digest had a final concentration and volume of $50 \mu \mathrm{g} \mathrm{mL} \mathrm{m}^{-1}$ and $500 \mu \mathrm{L}$, respectively. The digest was divided into $10 \mu \mathrm{L}$ aliquots and stored at $-32{ }^{\circ} \mathrm{C}$ until further use.

BSA standards were diluted with $\mathrm{ABC}(50 \mathrm{mM})$ to a final volume and concentration of $500 \mu \mathrm{L}$ and $50 \mu \mathrm{g} \mathrm{mL}^{-1}$, respectively. $2.5 \mu \mathrm{L}$ of $50 \mathrm{mM}$ DTT (freshly prepared in $50 \mathrm{mM} \mathrm{ABC}$ ) was added to the protein mixture and incubated at $60{ }^{\circ} \mathrm{C}(800 \mathrm{rpm})$ for $20 \mathrm{~min}$. Afterward, the solution was cooled, and $2.5 \mu \mathrm{L}$ of $200 \mathrm{mM}$ IAA (freshly prepared in $50 \mathrm{mM} \mathrm{ABC}$ ) was added. Incubation was carried out for $15 \mathrm{~min}$ at room temperature $(800 \mathrm{rpm})$ in the dark. Digestion was then accomplished by adding trypsin at an enzyme to substrate ratio of $1: 20(\mathrm{w} / \mathrm{w})$ overnight at $37^{\circ} \mathrm{C}$.

\subsubsection{NSE digest extractions}

The on-line MISPE method was evaluated by determining the recovery and linearity ( $1 / \mathrm{x}$ weighed) of an NSE digest. For recovery determination, NSE digest $100 \mathrm{ng} \mathrm{mL}^{-1}(10 \mu \mathrm{L})$ was diluted to a final concentration of $10 \mathrm{ng} \mathrm{mL}^{-1}$ by $50 \mathrm{mM} \mathrm{ABC}$ containing $25 \mathrm{nM}$ of digested BSA $(90 \mu \mathrm{L})(\mathrm{n}=3)$. For linearity determination, NSE digest $\left(50 \mu \mathrm{g} \mathrm{mL}^{-1}\right)$ was diluted to $10,25,50$ and $100 \mathrm{ng} \mathrm{mL}^{-1}(\mathrm{n}=3)$ with $50 \mathrm{mM}$ ABC containing $100 \mathrm{nM}$ digested BSA. The samples were injected $(2 \mu \mathrm{L})$ onto MIP 1 . The loading/extraction time was set to 10 min followed by gradient elution and analysis. To determine the extraction recovery, a control sample of $25 \mathrm{ng} \mathrm{mL}^{-1}$ of NSE digest in $50 \mathrm{mM} \mathrm{ABC}$ was analysed without the presence of the MIP column to determine the signal w/o MIP (=100\% recovery).

\subsection{Complex matrix extractions}

\subsubsection{RP-SPE treatment of digested serum}

For analyses of more complex matrices, RP-SPE treated serum was selected as a sample with complexity intermediate between a simple NSE digest and untreated digested serum. An in-house RPSPE treatment was performed as described previously [20]. Briefly, SPE tips were made in-house by punching out six discs of C8 $3 \mathrm{M}$ Empore material (Teknolab AS, Kolbotn, Norway) and packing them into the lower part of a $300 \mu \mathrm{L}$ bevel point pipette tip (VWR, Hanover, Germany) using a metal wire. The SPE material was activated with $100 \mu \mathrm{L}$ of MeCN followed by $100 \mu \mathrm{L}$ of $20 \mathrm{mM}$ FA. Digested serum $(50 \mu \mathrm{L})$ was transferred to the SPE tip. The tips were then washed with $100 \mu \mathrm{L}$ of $20 \mathrm{mM}$ FA before being eluted with $100 \mu \mathrm{L}$ of $\mathrm{MeCN} / 0.01 \%(\mathrm{v} / \mathrm{v}$ ) TFA in the ratio 80:20. The eluent was evaporated to dryness at $60{ }^{\circ} \mathrm{C}$ under $\mathrm{N}_{2}$ gas and reconstituted in $50 \mu \mathrm{L}$ of $50 \mathrm{mM} \mathrm{ABC}$.

\subsubsection{Human serum digestion}

Human serum was defrosted at $4^{\circ} \mathrm{C}$ for $1 \mathrm{~h}$. $200 \mu \mathrm{L}$ of serum was diluted 1:1 with $100 \mathrm{mM} \mathrm{ABC}$ before the addition of $200 \mathrm{mM}$ freshly prepared DTT $(10 \mu \mathrm{L})$ in $50 \mathrm{mM} \mathrm{ABC}$. The samples were incubated at $60{ }^{\circ} \mathrm{C}$ for $1 \mathrm{~h}$ and allowed to cool to room temperature before the addition of $10 \mu \mathrm{L}$ of freshly prepared $500 \mathrm{mM}$ IAA in $50 \mathrm{mM} \mathrm{ABC}$. The samples were incubated at room temperature (800 rpm) for $30 \mathrm{~min}$ in the dark prior to the addition of $70 \mu \mathrm{L}$ of
$10 \mathrm{mg} \mathrm{mL} \mathrm{m}^{-1}$ trypsin freshly prepared in $50 \mathrm{mM} \mathrm{ABC}$. The samples were digested overnight at $37^{\circ} \mathrm{C}$. Digested serum was frozen at $-20^{\circ} \mathrm{C}$ until required.

\subsubsection{Determination of recoveries in complex matrices}

Recoveries were evaluated in two complex matrices: NSE digest spiked into RP-SPE-treated digested human serum, and NSE digest spiked into digested serum. NSE digest (1875 $\left.\mathrm{ng} \mathrm{mL}^{-1} ; 10 \mu \mathrm{L}\right)$ was spiked into $90 \mu \mathrm{L}$ of RP-SPE-treated digested human serum or $1: 1$ diluted (in $50 \mathrm{mM} \mathrm{ABC}$ ) digested human serum for a final NSE digest concentration of $375 \mathrm{ng} \mathrm{mL}^{-1}$. To determine the extraction recoveries, a control sample of $187.5 \mathrm{ng} \mathrm{mL}-1$ of NSE digest in $50 \mathrm{mM}$ ABC was analysed without the presence of the MIP column to determine the signal w/o MIP (=100\% recovery). Following this, each sample type $(n=3)$ was injected onto the standard trap system and the relative signal intensity used to determine recoveries.

\subsubsection{Linearity, $L O D$ and $L O Q$}

Digested serum samples $(40 \mu \mathrm{L})$ were spiked with $5 \mu \mathrm{L}$ of various concentrations of digested NSE and $5 \mu \mathrm{L}$ of ELPLYR IS to final concentrations of $7.5,37.5,75,187.5$, and $375 \mathrm{ng} \mathrm{mL}^{-1} \mathrm{NSE}$ and

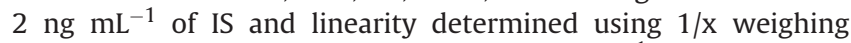
$(\mathrm{n}=3)$. The signal (S) of ELPLYR in the $7.5 \mathrm{ng} \mathrm{mL}^{-1}$ digested NSE in digested serum sample and the noise $(\mathrm{N})$ in the digested blank serum sample were used to calculate the LOQ $(\mathrm{S} / \mathrm{N}=10)$ and LOD $(\mathrm{S} / \mathrm{N}=3)$. The respective chromatograms are seen in Fig. $\mathrm{S} 2$.

\section{Results and discussion}

\subsection{Template design and optimisation of MIP syntheses}

Precipitation polymerization (PP) is a surfactant/stabilizer-free method for synthesising high quality crosslinked polymer microspheres in good yields, with good control over microsphere diameter, chemical functionality, and porous morphology. Typically, monomers are polymerised under dilute conditions in near-theta solvents, and products with narrow particle size distributions and bead diameters in the low-micron size range are formed. Such products are very well-suited for high-performance chemical separation work, including as stationary phases in LC and as SPE sorbents. It was for this reason that PP was the polymer synthesis method of choice in the present work.

ELPLYR (i.e., H-Glu-Leu-Pro-Leu-Tyr-Arg-OH) (Fig. 2A) was selected as the signature peptide for NSE based on a previous study which used this peptide as a surrogate for the determination of NSE from serum [18]. The peptide is specific for the $\gamma$-subunit of NSE which is present in both the heterodimeric $(\alpha \gamma)$ and homodimeric $(\gamma \gamma)$ form of NSE. The sequence ELPLYR (AA 127-132) is found on the outside of each the $\gamma$-monomer. Therefore, it may be more readily accessible to trypsin during digestion, improving cleavage efficiency, and generating a more intense signal during analysis. ELPLYR was not used as the template in the production of MIPs because bleeding/leaching of residual template from ELPLYR imprinted polymers would be expected to occur during exploitation of such MIPs for ELPLYR capture from tryptic digests, and bleeding/leaching of the template, where the template is structurally identical to the analyte, is unacceptable in ultra-trace analyses where the analyte concentration is very low (N.B. the reference levels of NSE in humans in $8.6 \mathrm{ng} \mathrm{mL}^{-1}$ ). A template analogue approach must, therefore, be used. In the design of a suitable template analogue for ELPLYR, some of the key considerations for the template analogue were: 1) Structural similarity to ELPLYR; 2) Cost and availability; 3) Solubility (in the porogens preferred for MIP production by PP); 4) Conformation and 
conformational rigidity; 5) Stability; 6) Functional groups available for templating. In a tryptic digest, any peptides present which contain C-terminal arginine are expected to bind appreciably and in a non-selective manner to a MIP prepared using a peptide template where the C-terminus of the template is arginine and this residue was targeted via a non-covalent molecular imprinting protocol. Thus it was decided to mutate the C-terminal arginine and build selectivity into the MIPs through amino acid residues other than the C-terminal arginine.

Based on the above considerations, the peptide used as a template analogue for the NSE signature peptide was Z-ELPLY[Nle], where $Z=C b z=$ Carboxybenzyl (a protecting group for the peptide $\mathrm{N}$-terminus), and Nle = norleucine (Fig. 2B). Points to note: 1 ) The introduction of the $\mathrm{Z}$ group, and the change of Arg to Nle, is expected to increase the solubility of the template in the porogens preferred for non-covalent molecular imprinting and PP; 2) The ionisable groups in the template analogue are all Brønsted acidic (the Brønsted basic groups present in H-ELPLYR-OH have been either protected or replaced); 3) The Glu side-chain and the Cterminal carboxylic acid are useful functional group handles for templating. Urea-based monomers for oxyanion binding are obvious functional monomer (FM) candidates; 4) The Tyr sidechain is also a potentially useful functional group handle for templating.

Establishing an appropriate ratio of FM to crosslinker is an important part of MIP design for SPE applications. If too little crosslinker is used then the polymers may not be porous in the wet state and access to binding sites may be impeded or prevented. On the other hand, whilst higher crosslinker levels are more likely to yield permanently porous materials, this may be at the expense of lower nominal binding capacity. Appropriate ratios of FM to crosslinker are normally arrived at through a process of trial-anderror. Given this background, the approach was to increase the relative amount of FM in the polymer network in a stepwise fashion in order to identify the best performing MIP, however when the FM content in the monomer feed was at a FM to crosslinker mol ratio of $1: 50$, the polymer particles formed through polymerization started to aggregate and high quality particles could no longer be obtained (probably due to the less effective self-stabilisation of DVB particles bearing urea moieties derived from the FM). Therefore, we decided to synthesise and evaluate two different sets of MIPs (and their corresponding NIPs) where the ratios of FM to crosslinker in the monomer feed were around 1:200 and 1:100.

\subsection{Method optimisation}

\subsubsection{Initial testing of MIP/NIP pairs 1 and 2}

The initial round of testing of the MIP columns focused on optimising the composition of the loading mobile phase and the flow rate. For this, each column was coupled directly to the MS using a single pump (Fig. 1B), and ELPLYR IS (5 nM) in $50 \mathrm{mM} \mathrm{ABC}$ injected $(2 \mu \mathrm{L})$ onto each column $(\mathrm{n}=3)$. For each column, the $\mathrm{k}$ values of ELPLYR IS were determined under a range of conditions (mobile phases of different elution strength were used, and the flow rates were varied) to establish the chromatographic conditions under which the MIP could bind the target strongly and selectively. To establish binding selectivity imparted into the MIPs through templating, retention factors were measured in parallel for non-imprinted control polymers, and then an imprinting factor (IF) calculated for each set of conditions. The initial optimisation of the composition of the loading mobile phase was performed using three different ratios of $20 \mathrm{mM}$ FA and $\mathrm{MeCN}(\mathrm{v} / \mathrm{v})$ since mixtures of $20 \mathrm{mM}$ FA and MeCN were shown to be effective mobile phases in earlier on-line MISPE work targeting tryptic peptides [12]. Similarly, the flow rates investigated $\left(20,30\right.$, and $\left.40 \mu \mathrm{L} \mathrm{min}{ }^{-1}\right)$ were based on earlier studies, with faster flow rates being preferred provided that shorter analysis times are not at the expense of analyte recovery.

The first mobile phase evaluated was 100\% 20 mM FA (data not shown). It was found that there was no observable MS signal at any flow rate on either of the MIPs or NIPs, even after $1 \mathrm{~h}$ of isocratic flow. This showed that both the MIPs and the NIPs retained the peptide strongly (no selectivity), which is unsurprising since nonselective binding is expected to dominate for divinylbenzenebased polymers when the mobile phase is fully aqueous. However, the peptide could be eluted off the polymers in the subsequent washing step, so the peptide is not bound irreversibly.

Subsequently, mixtures of $20 \mathrm{mM}$ FA and MeCN (at ratios 95:5 and 90:10 (v/v)) were evaluated as the loading mobile phase. The $\mathrm{k}$ values are high right across the board, and range from 14.4 to 21.7, which shows high affinity of the polymers for the target. When comparing the two MIPs (MIP 1 and MIP 2), MIP 1 has a consistently higher affinity for the target, and the difference between the polymers is greatest for the mobile phase with the highest organic

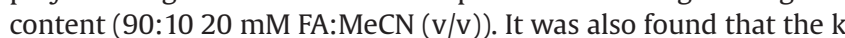
values were influenced very little by the flow rate, which suggested that the contact time between polymers and peptide was sufficiently long for all flow rates investigated. The highest $\mathrm{k}$ value was obtained for MIP 1 at a flow rate of $20 \mu \mathrm{L} \mathrm{min}^{-1}$ when using 95:5 $20 \mathrm{mM}$ FA:MeCN $(\mathrm{v} / \mathrm{v})$ as the mobile phase $(21.7 \pm 1.3)$. Under the same mobile phase and flow rate conditions, the corresponding NIP 1 had a $\mathrm{k}$ value of $19.8 \pm 0.3$, which gives an imprinting factor of 1.1 . Whilst this is a modest imprinting factor, the affinity of MIP 1 for the target is high, and high levels of non-specific binding to the non-imprinted sorbents arise due to a combination of hydrophobic effects and hydrogen bonding interactions between carboxylate groups in the target and the pendent urea moieties that are distributed randomly throughout the polymers.

For MIP 2 and NIP 2, the k values were also found to be highest when using 95:5 $20 \mathrm{mM}$ FA:MeCN $(\mathrm{v} / \mathrm{v})$ as the loading mobile phase at a flow rate of $20 \mu \mathrm{L} \mathrm{min} \operatorname{mon}^{-1}(20.1 \pm 0.7$ and $19.40 \pm 0.3$, respectively), however, the IF value was lower ( $I F=1.04$.). Thus, whilst affinity remains high there is a fall in selectivity. When the amount of MeCN in the mobile phase was increased from 5\% to $10 \%$ (i.e., to 90:10 $20 \mathrm{mM} \mathrm{FA:MeCN} \mathrm{v/v)} \mathrm{the} \mathrm{k}$ values decreased somewhat for all MIPs and NIPs which is consistent with a disruption of non-selective binding via hydrophobic effects. For all the mobile phases and flow rates evaluated, there is no significant difference (two-sided T-test, $\mathrm{P} \geq 0.05$ ) in $\mathrm{k}$ values between any MIP and the corresponding NIP except for MIP 1 and NIP 1 at a flow rate of $40 \mu \mathrm{L} \mathrm{min}{ }^{-1}$ when using 90:10 $20 \mathrm{mM} \mathrm{FA:MeCN} \mathrm{(v/v)} \mathrm{as} \mathrm{the} \mathrm{mobile}$ phase $(P=0.0009)$. However, with this mobile phase, the $\mathrm{k}$ values for MIP $1(16.5 \pm 0.3)$ is up to $25 \%$ lower than that obtained when using the 95:5 $20 \mathrm{mM}$ FA:MeCN (v/v) mobile phase, and higher retention factors are normally preferable when working with complex samples where there will be more competition for binding sites.

In this initial round of testing, MIP 1 turned out to be the most promising polymer; it had a high affinity for the target together with a degree of selectivity. To improve the selectivity it was decided to fine-tune the mobile phase composition in the range 100:0 to 95:5 $20 \mathrm{mM}$ FA:MeCN (v/v). For this part of the MISPE optimisation, the flow rate was fixed at $20 \mu \mathrm{L} \mathrm{min}{ }^{-1}$.

\subsubsection{Fine-tuning of the loading of MIP 1}

The fine-tuning of the mobile phase composition for the loading of MIP 1 was performed using mobile phases containing 95:5, 96:4 $97: 3,98: 2$, and 99:1 ratios of $20 \mathrm{mM} \mathrm{FA:MeCN} \mathrm{(v/v).} \mathrm{Similarly} \mathrm{to} \mathrm{the}$ results obtained when the mobile phase was 100\% 20 mM FA (i.e., under fully aqueous loading conditions), ELPLYR was not eluted 
within $1 \mathrm{~h}$ when using 98:2 and 99:1 $20 \mathrm{mM} \mathrm{FA:MeCN} \mathrm{(v/v)} \mathrm{as} \mathrm{the}$ mobile phase (data not shown). However, for the three other mobile phase compositions investigated, there was a clear trend of increasing $\mathrm{k}$ values with decreasing MeCN. At 95:5, 96:4 and 97:3 $20 \mathrm{mM}$ FA:MeCN (v/v), the $\mathrm{k}$ values recorded for MIP 1 increased steadily from $19.5 \pm 0.1$ to $20.3 \pm 0.4$ to $23.8 \pm 0.6$, respectively (Fig. 3). NIP 1 showed a similar, but weaker, trend, with $\mathrm{k}$ values of $18.6 \pm 0.1,19.1 \pm 0.3$, and $20.6 \pm 0.4$, respectively. For all three of these mobile phases a significant difference between the MIP 1 and NIP $1 \mathrm{k}$ values was seen $(\mathrm{P}=0.014-0.0005)$. When loading with 97:3 $20 \mathrm{mM}$ FA:MeCN (v/v), the highest $\mathrm{k}$ value on MIP 1 was obtained (23.8), giving an IF of 1.2. This is the highest IF obtained across all the conditions tested, and is slightly higher than the IF reported for earlier work concerning on-line-MISPE of tryptic peptides [12]. Accordingly, for the further optimisation work the mobile phase was fixed as 97:3 $20 \mathrm{mM} \mathrm{FA:MeCN}(\mathrm{v} / \mathrm{v})$, since strong affinity and useful selectivity were apparent under these conditions.

\subsubsection{Loading time (extraction time)}

The loading (extraction) time during on-line analysis is critical for ensuring maximal retention of the analyte of interest while simultaneously reducing the sample complexity as much as possible before analysis. This process ensures maximum sensitivity due to reduced matrix effects in the MS. A pragmatic balance must be struck between the duration of the extraction step, the recovery of the target analyte, and the overall analysis time. To evaluate the loading (extraction) time, MIP 1 was used as a trap column and coupled to a triple quadrupole MS, as described in the Experimental (Fig. 1A).

The recovery of ELPLYR when using a 5 min loading time was $95.3 \pm 1.2 \%$ (Table 1 ). The recovery increased to $100 \%$ when loading for 7.5 min or longer. Thus MIP 1 can retain ELPLYR exceptionally well (there was no loss of peptide after $20 \mathrm{~min}$, which equates to approximately 40 column volumes). However, to investigate in more detail the selectivity of the MIP for ELPLYR over other peptides, digested BSA was loaded onto the MIP.

The recovery of seven tryptic BSA peptides was determined using three different loading times $(5,10$, and 20 min; Table 2). With a loading time of $5 \mathrm{~min}$, the recoveries ranged from 9.5 to $107.5 \%$, with an average recovery of $66.5 \pm 12.4 \%$. When the loading time was doubled to $10 \mathrm{~min}$, the average recovery was $59.3 \pm 6.3 \%$. Increasing the loading time yet further to 20 min resulted in an average recovery of $55.0 \pm 5.4 \%$. An average recovery of $55-67 \%$ for

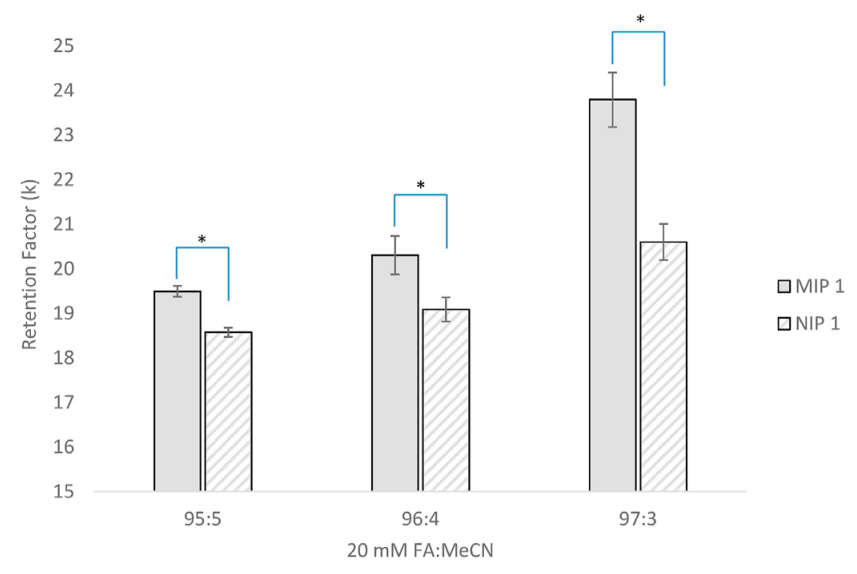

Fig. 3. Retention factors (k) of MIP 1 and NIP 1 at $20 \mu \mathrm{L} \mathrm{min}-1$ isocratic flow with mobile phases 95:5, 96:4 and 97:3 mM FA:MeCN (v/v). $* \mathrm{P} \leq 0.05$.
Table 1

Recoveries of ELPLYR tryptic peptide using loading (extraction) times of 5, 7.5, 10 12.5 , and $20 \mathrm{~min}$.

\begin{tabular}{lll}
\hline Extraction time $(\mathrm{min})$ & ELPLYR Recovery $(\%)$ & RSD $(\%)$ \\
\hline 5 & 95.3 & 1.4 \\
7.5 & 103 & 2.9 \\
10 & 100 & 5.0 \\
12.5 & 105 & 9.5 \\
20 & 102 & 4.4 \\
\hline
\end{tabular}

the seven tryptic BSA peptides, compared to essentially quantitative recovery of the target, implies moderate selectivity for ELPLYR, however only one peptide was retained as strongly as ELPLYR, LGEYGFQNALIVR, and this is most likely due to its isoelectric point ( $\mathrm{pI}=6.1$ ) being comparable to ELPLYR $(\mathrm{pI}=6.0$ ). Since there were no significant differences in non-specific binding as a function of extraction time, 10 min was selected as the optimum extraction time since the recovery of ELPLYR was 100\% even after washing with over 20 column volumes of mobile phase.

\subsection{Initial evaluation of analytical performance}

The initial evaluation of the method involved determining the recovery and linearity to establish the binding capacity of the MIP in simple matrices. This gives an insight into the potential downstream compatibility of the MIP with extractions of complex matrices. The extraction recovery of ELPLYR was determined to $100.4 \pm 5.0 \%$ in a sample of digested NSE in $50 \mathrm{mM} \mathrm{ABC}$ containing digested BSA (10 times molar amount). Linearity was determined by injecting digested NSE in the range $10-100 \mathrm{ng} \mathrm{mL}^{-1}$ in $50 \mathrm{mM}$ ABC containing a $100 \mathrm{nM}$ digested BSA $(\mathrm{n}=3)$ onto the MIP column. Linear regression was obtained by plotting the intensity of ELPLYR vs. the added concentration. The correlation value $\left(\mathrm{R}^{2}=0.99\right)$ was within acceptable limits and is higher than for previously reported on-line MISPE columns [12].

\subsection{Extractions from complex matrices}

Extractions from matrices of increasing complexity were evaluated to determine the compatibility of the on-line MISPE method with real biological samples and to verify the need to use a MIP for extraction when analysing complex samples. The latter was investigated as the MIP and NIP had shown similar retention factors of ELPLYR in extractions from simple matrices. Digested NSE was spiked into digested human serum pre-treated by off-line RP-SPE and also into untreated digested human serum; the recovery of ELPLYR was then determined for the two sample types (Fig. 4) using the MIP as a trap column; the latter sample was also analysed using the NIP as a trap column. When using the MIP as a trap column, the extraction recovery from RP-SPE treated serum was $100.8 \pm 6.2 \%$. Therefore, the extraction efficiency was comparable to those in a simple matrix. MIP extractions from the most complex matrix (digested serum), on the other hand, showed a lower but still acceptable recovery of ELPLYR (61.6 $\pm 1.9 \%)$. In comparison, when the NIP was used to extract digested NSE from digested serum, the recovery dropped to $25.2 \pm 1.7 \%$, demonstrating the effect of the imprinting in extractions from complex matrices.

The high recovery of ELPLYR from RP-SPE treated serum demonstrates that the MIP can enrich low abundant ELPLYR even when it is in the presence of high numbers of non-specific peptides. The recovery of ELPLYR from untreated, 1:1 diluted serum is lower than for the less complex matrices. The recovery is, however, within the ranges reported for other MIPs targeting proteins: A MISPE method targeting the signature peptide NLLGLIEAK (from the SCLC 
Table 2

Recoveries of seven BSA tryptic peptides loaded for 5,10 , and $20 \min (n=3)$.

\begin{tabular}{|c|c|c|c|c|c|c|}
\hline \multirow[b]{2}{*}{ Peptide } & \multicolumn{2}{|l|}{$5 \mathrm{~min}$} & \multicolumn{2}{|l|}{$10 \mathrm{~min}$} & \multicolumn{2}{|l|}{$20 \mathrm{~min}$} \\
\hline & Recovery (\%) & RSD (\%) & Recovery (\%) & RSD (\%) & Recovery (\%) & RSD (\%) \\
\hline DAELGSFLYEYSR & 78.9 & 37.4 & 80.3 & 1.1 & 73.4 & 12.4 \\
\hline YICDNQDTISSK & 83.9 & 6.5 & 88.9 & 3.8 & 82.2 & 9.1 \\
\hline HLVDEPQNLIL & 94.7 & 0.8 & 86.9 & 12.3 & 79.5 & 3.0 \\
\hline AEFVEVTK & 45.0 & 54.0 & 19.8 & 11.6 & 11.9 & 114.6 \\
\hline LVTDLTK & 9.5 & 173.2 & 0 & $\mathrm{n} / \mathrm{a}$ & 0 & $\mathrm{n} / \mathrm{a}$ \\
\hline HPEYAVSVLLR & 46.1 & 19.0 & 41.9 & 9.2 & 42.9 & 11.9 \\
\hline LGEYGFQNALIVR & 107.5 & 14.2 & 97.0 & 5.8 & 95.1 & 0.1 \\
\hline Average & 66.5 & 39.5 & 59.3 & 6.3 & 51.9 & 21.6 \\
\hline
\end{tabular}

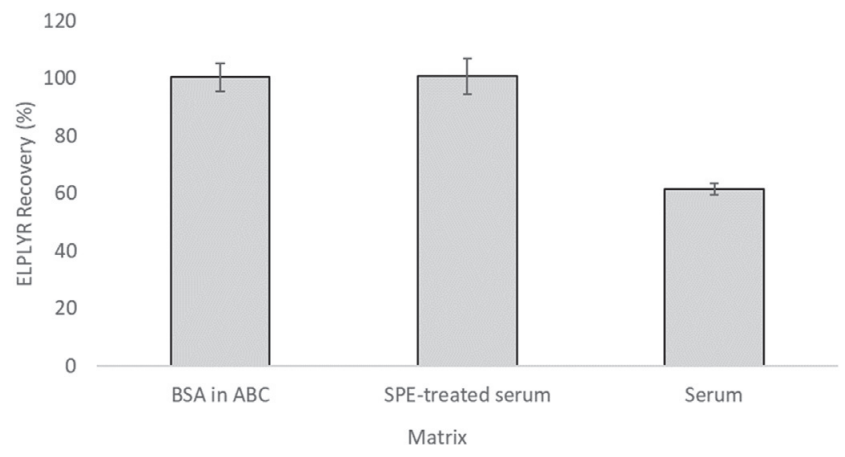

Fig. 4. Recoveries of ELPLYR after spiking $5 \mathrm{ng} \mathrm{mL}^{-1}$ digested NSE to matrices of increasing complexity ( $50 \mathrm{mM}$ ABC containing $50 \mathrm{nM}$ digested BSA, SPE-treated digested human serum, and untreated digested human serum, $n=3$ ).

biomarker ProGRP) gave a recovery of $<25 \%$ in precipitated serum [17], and an epitope imprinted on-line MISPE method targeting the sequence MIQRTPKIQ from beta2-microglobulin showed recoveries of $\geq 83 \%$ in spiked serum samples ( $\mu \mathrm{g} \mathrm{mL} \mathrm{m}^{-1}$ range). An NSEimprinted electrochemical sensor based assay reported recoveries between approximately $96-100 \%$ in 1:100 diluted serum [19]. Most likely, the dilution factor of the latter method reduces the complexity sufficiently to ensure complete recoveries. Nevertheless, MIP 1 has excellent compatibility with complex matrices, such as minimally diluted serum, and is therefore likely to be able to analyse NSE in serum at levels below the clinical reference range. In addition, since the recovery drops when the MIP is replaced by the NIP when extracting complex samples, then imprinting is likely to play an essential role in the extraction. The lack of selective binding sites in the NIP leads to the drop in recovery as ELPLYR must compete with the myriad tryptic peptides in the digested serum for interaction with the solid-phase.

The efficient extraction of ELPLYR for complex matrices using MIP demonstrates the value of MIPs as potential tools in diagnostics as well as aiding in biomarker validation.

\subsection{Analytical evaluation in complex matrices}

The analytical performance was evaluated by determining the linearity, LOD, and LOQ of NSE in digested human serum $(\mathrm{n}=3)$. The method is linear in the disease range (3.4-344.2 $\left.\mathrm{ng} \mathrm{mL}^{-1}\right)$ [21], and the linear regression was within acceptable limits $\left(R^{2}>0.99\right.$, slope 0.0026 , intercept -0.0019$)$. The LOD and LOQ were estimated to be $1.8(\mathrm{~S} / \mathrm{N}=3)$ and $6.0 \mathrm{ng} \mathrm{mL}^{-1}(\mathrm{~S} / \mathrm{N}=10)$, respectively, which is a very significant finding because the LOQ is below the median reference levels in humans $\left(8.6 \mathrm{ng} \mathrm{mL}^{-1}\right.$ ) [22]. Therefore, the online MISPE method has great potential in NSE analysis and quantification, subject to efficient digestion of the complex samples.
Presently, the digestion efficiency of NSE is approximately 50\%, therefore further improvements in the digestion could yield up to two-fold reductions in the LOD and LOQ. Furthermore, the use of more contemporary MS systems in combination with nanoflow LC are expected to reduce the LOD and LOQ yet further. Irrespective of the further refinements that are possible, the on-line MISPE protocol developed and presented here can quantify NSE below the reference level in human serum. The good performance of MIPs in contact with complex matrices demonstrates the potential value MIPs have in diagnostics.

\section{Conclusions}

The present work demonstrates the successful design, synthesis, and exploitation of robust synthetic receptors targeting the signature peptide of the biomarker NSE for the diagnosis of SCLC. Two MIPs were synthesized in a convenient beaded format using precipitation polymerization and packed into columns for on-line MISPE-LC-MS/MS. Initial optimisation work revealed that the polymers had high affinity for the target, and further optimisation of the MISPE protocol enabled conditions to be established where the target could be extracted efficiently from water-rich samples. Thereafter, the analytical potential of the most promising synthetic receptor (MIP 1) was evaluated with complex matrices. The on-line extraction method had acceptable recoveries, excellent linearity, and LODs and LOQs for NSE which were in the low $\mathrm{ng} \mathrm{mL}^{-1}$ range and, very significantly, below the human reference level in serum (which is $8.6 \mathrm{ng} \mathrm{mL}^{-1}$ ). The low cost and stability of the MIP, combined with the automated on-line MISPE enrichment and LCMS/MS of the target molecule, is very promising for SCLC diagnosis in particular, and the low-cost analysis of analytes in complex matrices in general, as well as for aiding biomarker validation.

\section{Declaration of competing interest}

The authors declare that they have no known competing financial interests or personal relationships that could have appeared to influence the work reported in this paper.

\section{Acknowledgements}

This work was supported by the Horizon 2020 Research Framework Programme of the European Commission BioCapture project (722171). We thank Dr. R. Trones from G\&T Septech AS, Norway, for assisting with the packing of the MIPs and NIPs into columns.

\section{Appendix A. Supplementary data}

Supplementary data to this article can be found online at https://doi.org/10.1016/j.aca.2020.10.022. 


\section{References}

1] G. Poste, Bring on the biomarkers, Nature 469 (7329) (2011) 156-157.

[2] R.M. Lequin, Enzyme immunoassay (EIA)/enzyme-linked immunosorben assay (ELISA), Clin. Chem. 51 (12) (2005) 2415-2418.

[3] K. Haupt, K. Mosbach, Molecularly imprinted polymers and their use in biomimetic sensors, Chem. Rev 100 (7) (2000) 2495-2504.

[4] A. Mujahid, N. Iqbal, A. Afzal, Bioimprinting strategies: from soft lithography to biomimetic sensors and beyond, Biotechnol. Adv. 31 (8) (2013) 1435-1447.

[5] A. Poma, A.P. Turner, S.A. Piletsky, Advances in the manufacture of MIP nanoparticles, Trends Biotechnol. 28 (12) (2010) 629-637.

[6] G. Vasapollo, R.D. Sole, L. Mergola, M.R. Lazzoi, A. Scardino, S. Scorrano, G. Mele, Molecularly imprinted polymers: present and future prospective, Int. J. Mol. Sci. 12 (9) (2011) 5908-5945.

[7] M.X. Li, X.H. Wang, L.M. Zhang, X.P. Wei, A high sensitive epitope imprinted electrochemical sensor for bovine serum albumin based on enzyme amplifying, Anal. Biochem. 530 (2017) 68-74.

[8] J.Z. Hilt, M.E. Byrne, Configurational biomimesis in drug delivery: molecular imprinting of biologically significant molecules, Adv. Drug Deliv. Rev. 56 (11) (2004) 1599-1620.

[9] E. Saridakis, S. Khurshid, L. Govada, Q. Phan, D. Hawkins, G.V. Crichlow, E. Lolis, S.M. Reddy, N.E. Chayen, Protein crystallization facilitated by molecularly imprinted polymers, Proc. Natl. Acad. Sci. U.S.A. 108 (27) (2011) $11081-11086$.

[10] L. Chen, X. Wang, W. Lu, X. Wu, J. Li, Molecular imprinting: perspectives and applications, Chem. Soc. Rev. 45 (8) (2016) 2137-2211.

[11] W.J. Cheong, S.H. Yang, F. Ali, Molecular imprinted polymers for separation science: a review of reviews, J. Separ. Sci. 36 (3) (2013) 609-628.

[12] C. Rossetti, M.A. Świtnicka-Plak, T.G. Halvorsen, P.A. Cormack, B. Sellergren, L. Reubsaet, Automated protein biomarker analysis: on-line extraction of clinical samples by molecularly imprinted polymers, Sci. Rep. 7 (2017) 44298

[13] A. Rachkov, M. Hu, E. Bulgarevich, T. Matsumoto, N. Minoura, Molecularly imprinted polymers prepared in aqueous solution selective for [Sar 1, Ala 8] angiotensin II, Anal. Chim. Acta 504 (1) (2004) 191-197.

[14] F. Yang, D. Deng, X. Dong, S. Lin, Preparation of an epitope-imprinted polymer with antibody-like selectivity for beta2-microglobulin and application in serum sample analysis with a facile method of on-line solid-phase extraction coupling with high performance liquid chromatography, J. Chromatogr. A coupling with high

15] M. Kempe, K. Mosbach, Separation of amino acids, peptides and proteins on molecularly imprinted stationary phases, J. Chromatogr. A 691 (1-2) (1995) 317-323.

[16] R. Boysen, Advances in the development of molecularly imprinted polymers for the separation and analysis of proteins with liquid chromatography, J. Separ. Sci. 42 (1) (2019) 51-71.

[17] C. Rossetti, A. Abdel Qader, T.G. Halvorsen, B.r. Sellergren, L. Reubsaet, Antibody-free biomarker determination: exploring molecularly imprinted polymers for pro-gastrin releasing peptide, Anal. Chem. 86 (24) (2014) $12291-12298$.

[18] S.B. Torsetnes, S.G. Løvbak, C. Claus, H. Lund, M.S. Nordlund, E. Paus, T.G. Halvorsen, L. Reubsaet, Immunocapture and LC-MS/MS for selective quantification and differentiation of the isozymes of the biomarker neuronspecific enolase in serum, J. Chromatogr. B 929 (2013) 125-132.

[19] X. Wang, Y. Wang, X. Ye, T. Wu, H. Deng, P. Wu, C. Li, Sensing platform for neuron specific enolase based on molecularly imprinted polymerized ionic liquids in between gold nanoarrays, Biosens. Bioelectron. 99 (2018) 34-39.

[20] C. Rosting, A. Gjelstad, T.G. Halvorsen, Water-soluble dried blood spot in protein analysis: a proof-of-concept study, Anal. Chem. 87 (15) (2015) 7918-7924.

[21] D. Yu, K. Du, T. Liu, G. Chen, Prognostic value of tumor markers, NSE, CA125 and SCC, in operable NSCLC Patients, Int. J. Mol. Sci. 14 (6) (2013) $11145-11156$.

[22] T. Esscher, L. Steinholtz, J. Bergh, E. Nöu, K. Nilsson, S. Påhlman, Neurone specific enolase: a useful diagnostic serum marker for small cell carcinoma of the lung, Thorax 40 (2) (1985) 85-90. 

Electronic Supplementary Material

Facilitating Serum Determination of Neuron Specific Enolase at Clinically Relevant Levels by Coupling On-line Molecularly Imprinted Solid-Phase Extraction to LC-MS/MS

Nicholas McKitterick, Tugrul Cem Bicak, Peter A. G. Cormack, Léon Reubsaet and Trine Grønhaug Halvorsen 



\section{Content of Electronic Supplementary Material}

Page S-2 Table S1: Transitions for BSA peptides monitored by a triple quadrupole mass spectrometer as referred to in MATERIALS AND METHODS in the manuscript.

Page S-3 Table S2. Sedimentation analysis of the polymers in the solvents $\mathrm{MeCN}, \mathrm{MeOH}$ and acetone.

Page S-4 Table S3. Peak symmetry analysis of each polymer column and respective back pressures.

Page S-4 Figure S1. Optical microscopy image of a newly packed MIP 1 column (1.5 ID x 5 $\mathrm{mm})$

Page S-5 Figure S2. Overlaid blank digested serum extraction and sample extraction (37.5 ng $\mathrm{mL}^{-1} \mathrm{NSE}$ in digested serum) used for LOD and LOQ estimations

\section{Page S-5 SUPPLEMENTARY RESULTS AND DISCUSSION}

Page S-7 Table S4. Synthesis conditions and isolated yields of the polymers.

Page S-8 $\quad$ Figure S3. SEM micrographs of MIP 1 (a), NIP 1 (b), MIP 2 (c) and NIP 2 (d)

Page S-9 Table S5. Particle sizes and particle size distributions of the polymer microspheres.

Page S-10-11 Figure S4. Nitrogen sorption analysis data for MIP 1 (A), NIP 1 (B), MIP 2 (C), NIP 2 (D).

Page S-12 Table S6. Specific surface areas, specific pore volumes, and mean pore sizes of the MIPs and NIPs.

Page S-13 REFERENCES 


\section{BOVINE SERUM ALBUMIN MASS SPECTROMETRY TRANSITIONS}

Table S1. Transitions for BSA peptides monitored by a triple quadrupole mass spectrometer (ESI positive mode).

\begin{tabular}{lccc}
\hline Peptide & Precursor lon mass $(\mathrm{m} / \mathrm{z})$ & Product ion mass $(\mathrm{m} / \mathrm{z})$ & Collision energy $(\mathrm{V})$ \\
\hline LVTDLTK & 395.240 & 577.360 & 30 \\
AEFVEVTK & 461.750 & 722.430 & 30 \\
HPEYAVSLLR & 642.360 & 920.610 & 30 \\
HLVDEPQNLIK & 653.360 & 1055.590 & 30 \\
YICDNQDTISSK & 722.820 & 1168.450 & 30 \\
LGEYGFQNALIVR & 740.400 & 1017.530 & 30 \\
DAFLGSFLYEYSR & 784.380 & 1121.560 & 30 \\
\hline
\end{tabular}




\section{EVALUATION OF COLUMN PACKING}

\section{Sedimentation Analysis}

Each polymer (6 mg per analysis) was suspended in $500 \mu \mathrm{L}$ portions of three different solvents: MeCN, $\mathrm{MeOH}$ and acetone $(\mathrm{n}=3)$ in a $2 \mathrm{~mL}$ glass vial. The slurries were ultrasonicated for $10 \mathrm{~min}$. followed by vortexing for $1 \mathrm{~min}$. Each slurry was left to stand until the particles began to sediment to the bottom of the vial. Each polymer was evaluated in terms of sedimentation time (Table S2). It was found that all polymers sedimented rapidly in acetone, the NIPs sedimented rapidly in $\mathrm{MeOH}$, and all polymers sedimented slowly in MeCN. Therefore, MeCN was chosen as the solvent for the slurry packing of columns, since this ensures that a uniform slurry is placed into the packing chamber, leading to more uniform, reproducible packing.

Table S2. Sedimentation analysis of the polymers in the solvents $\mathrm{MeCN}, \mathrm{MeOH}$ and acetone.

\begin{tabular}{|c|c|c|c|c|c|c|}
\hline \multirow[b]{2}{*}{ Polymer } & \multicolumn{2}{|c|}{ MeCN } & \multicolumn{2}{|c|}{$\mathrm{MeOH}$} & \multicolumn{2}{|c|}{ Acetone } \\
\hline & $2 \mathrm{~min}$. & $5 \mathrm{~min}$. & $2 \mathrm{~min}$. & $5 \mathrm{~min}$. & $2 \mathrm{~min}$. & $5 \mathrm{~min}$. \\
\hline MIP 1 & & & & & & \\
\hline NIP 1 & & & & & & \\
\hline MIP 2 & & & & & & \\
\hline NIP 2 & & & & & & \\
\hline
\end{tabular}

\section{Column packing evaluation}

Following the packing of the polymers, each column was evaluated under an optical microscope to identify any packing irregularities (Fig. S3). Subsequently, each column was evaluated by LC-UV for peak symmetry analysis. Naphthalene (at a concentration of $1 \mathrm{mg} / \mathrm{mL}$ in 50:50 v/v water:MeCN) was selected as the tracer molecule. A single pump was attached to the column and set at a flow rate of 20 $\mu \mathrm{L} / \mathrm{min}$ (60:40 MeCN:water), followed by direct injection of naphthalene $(20 \mu \mathrm{L}, \mathrm{n}=3)$. UV detection at 
$254 \mathrm{~nm}$ was used to determine peak asymmetry (Table S2). All of the columns had acceptable peak asymmetry (i.e., Asymmetry factor, $\left.\mathrm{A}_{s}<1.5\right)$ (Table $\mathrm{S} 3$ ).

Table S3. Peak symmetry analysis of each packed column and respective backpressures.

\begin{tabular}{lcc}
\hline Polymer in Column & $\mathbf{A}_{\mathbf{s}}(\mathrm{RSD}, \%)$ & Back pressure (PSI) \\
\hline MIP 1 & $1.21(1.03)$ & 12 \\
NIP 1 & $1.07(1.87)$ & 13 \\
MIP 2 & $1.23(11.8)$ & 12 \\
NIP 2 & $1.30(9.98)$ & 24 \\
\hline
\end{tabular}

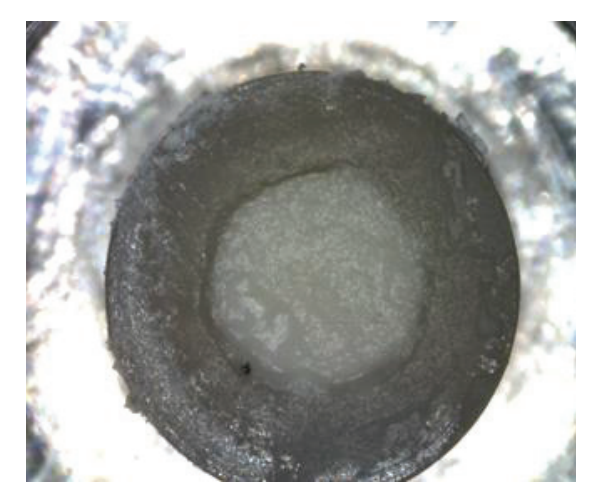

Figure S1. Optical microscopy image of a newly packed MIP 1 column (5 mm x $1.5 \mathrm{~mm}$ ID) 


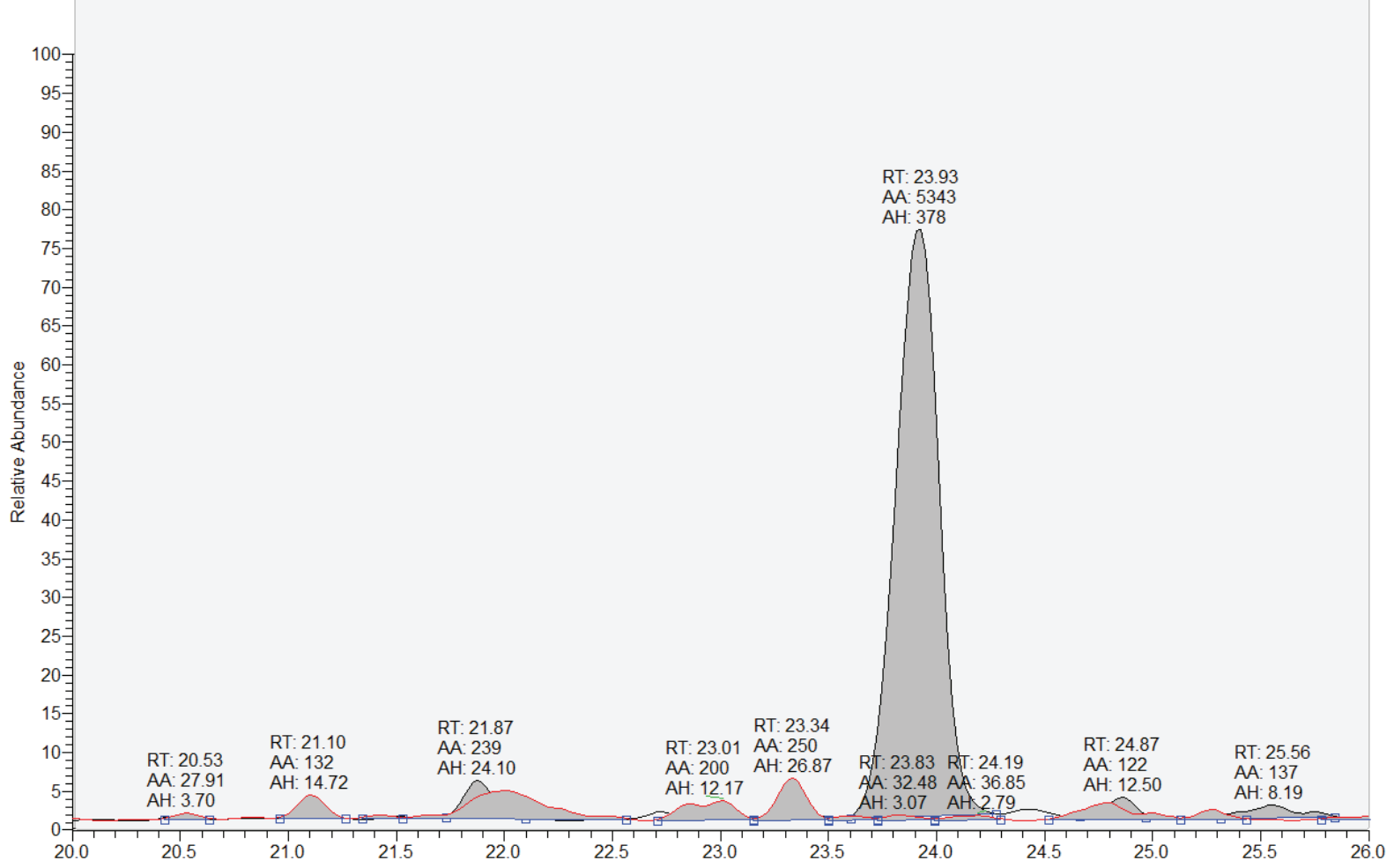

Figure S2. Overlaid blank digested serum extraction and sample extraction (37.5 $\mathrm{ng} \mathrm{mL}^{-1} \mathrm{NSE}$ in digested serum) used for LOD and LOQ estimations

\section{SUPPLEMENTARY RESULTS AND DISCUSSION}

Since acetonitrile is a suitable solvent for the synthesis of divinylbenzene-based polymer microspheres via precipitation polymerization, the solubility of the template in acetonitrile was investigated first. It was found that the template was soluble in acetonitrile at concentrations $>2 \mathrm{mg} / \mathrm{mL}$, hence acetonitrile can be used as a solvent (or major co-solvent) for preparation of the MIPs.

Initially, two MIP/NIP pairs were synthesized according to a literature method,[1] with Z-Glu-Leu-ProLeu-Tyr-Nle-OH as a template. However, aggregated irregular particles that were smaller than 1 micron were obtained by this procedure, which was ascribed to the presence of DMSO as a co-solvent.

For the synthesis of divinylbenzene-based polymer microspheres via precipitation polymerization, other solvents have been used in combination with acetonitrile, as reported in the literature.[2, 3] 
Examples include toluene and THF. Uniform particles between 2-5 microns were obtained by using 10$20 \%$ THF as a co-solvent in a photo-initiated PP[3]. Thus, THF was used in combination with acetonitrile as solvents for the templating of Z-Glu-Leu-Pro-Leu-Tyr-Nle-OH under precipitation polymerisation conditions, with DVB-80 as crosslinker (conc. $5 \% \mathrm{v} / \mathrm{v}$ relative to porogen). The THF/ MeCN ratio was set to $1 / 8(\mathrm{v} / \mathrm{v})$. The overall reaction conditions are summarised in Table S4. 
Table S4. Synthesis conditions and isolated yields of the polymers.

\begin{tabular}{|c|c|c|c|c|c|}
\hline & Components & MIP 1 & NIP 1 & MIP 2 & NIP 2 \\
\hline \multirow{8}{*}{ 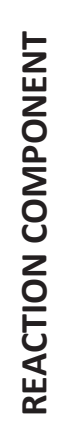 } & DVB-80 (mmol) & 7.74 & 7.74 & 7.74 & 7.74 \\
\hline & Urea monomer TPVU ( $\mu \mathrm{mol})$ & 70 & 70 & 35 & 35 \\
\hline & Template (Z-ELPLY[Nle]) ( $\mu \mathrm{mol})$ & 7.5 & - & 7.5 & - \\
\hline & PMP ( $\mu \mathrm{mol})$ & 10 & - & 10 & - \\
\hline & TBA.OH (1 M) ( $\mu \mathrm{mol})$ & 70 & 70 & 35 & 35 \\
\hline & AIBN ( $\mu \mathrm{mol})$ & 120 & 120 & 120 & 120 \\
\hline & MeCN & $20 \mathrm{~mL}$ & $20 \mathrm{~mL}$ & $20 \mathrm{~mL}$ & $20 \mathrm{~mL}$ \\
\hline & THF & $2.5 \mathrm{~mL}$ & $2.5 \mathrm{~mL}$ & $2.5 \mathrm{~mL}$ & $2.5 \mathrm{~mL}$ \\
\hline & Reaction time & $48 \mathrm{~h}$ & $48 \mathrm{~h}$ & $48 \mathrm{~h}$ & $48 \mathrm{~h}$ \\
\hline & Reaction temperature & $60^{\circ} \mathrm{C}$ & $60^{\circ} \mathrm{C}$ & $60^{\circ} \mathrm{C}$ & $60^{\circ} \mathrm{C}$ \\
\hline \multirow{5}{*}{ 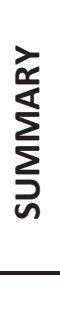 } & Monomer loading (w/v) & $\sim 5 \%$ & $\sim 5 \%$ & $\sim 5 \%$ & $\sim 5 \%$ \\
\hline & AIBN (w/w relative to total monomer) & $2 \%$ & $2 \%$ & $2 \%$ & $2 \%$ \\
\hline & THF/MeCN ratio(v/v) & $1 / 8$ & $1 / 8$ & $1 / 8$ & $1 / 8$ \\
\hline & Template/Functional Monomer ratio (mol/mol) & $1 / 9$ & 0 & $1 / 9$ & 0 \\
\hline & YIELD OF POLYMER & $49 \%$ & $45 \%$ & $51 \%$ & $48 \%$ \\
\hline
\end{tabular}

Four polymers (two imprinted polymers, MIP 1 and MIP 2, and their corresponding non-imprinted polymer, NIP 1 and NIP 2) were prepared in a microsphere format using the conditions outlined in Table S4, with Z-ELPLY[Nle]-OH as the template. Two different template to functional monomer mole ratios were used (1:4.7 and 1:9.3), to discern any influence on the molecular recognition capabilities of the products. It is noteworthy that the yields of products were considerably higher than the yields of photo-polymerisations reported in the literature.[3] This can be attributed to the low initiation efficiency of the photo-polymerisations, which is mainly due to the suspended particles formed during polymerisation interfering with the penetration of light into the reaction vessel. 


\section{CHARACTERIZATION OF MIPs AND NIPs}

\section{SEM}

SEM analyses were performed on a Cambridge Instruments Stereoscan 90 scanning electron microscope. Samples were sputter-coated with gold using a Polaron SC500A Sputter Coater for 4 minutes under argon. Image analysis of the SEM micrographs was performed using Image J47 software, on a population of 100 microspheres (Fig. S3).
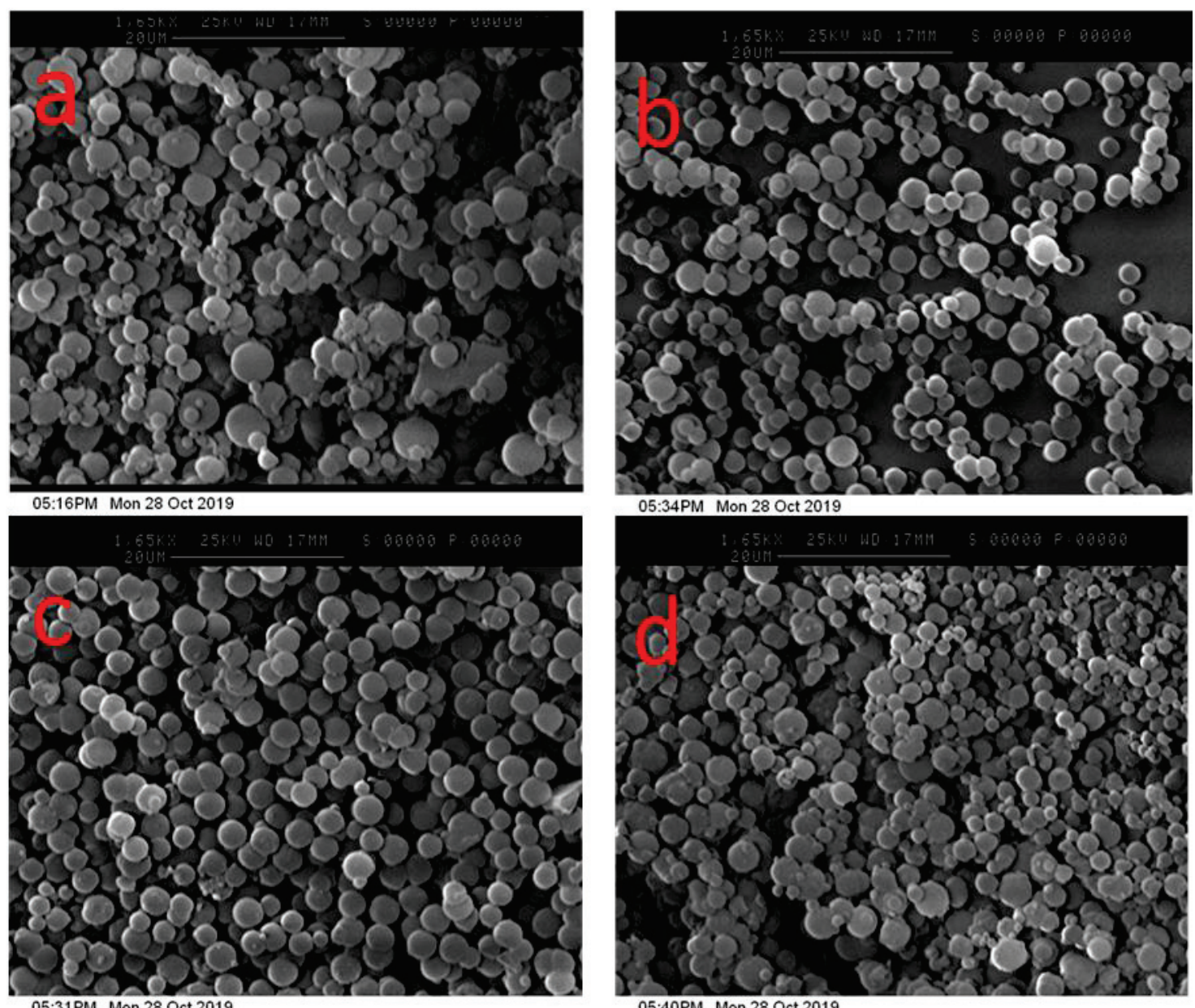

Figure S3. SEM micrographs of MIP 1 (a), NIP 1 (b), MIP 2 (c) and NIP 2 (d)

As expected, polymer particles with low mean particle diameters (from 2-3 $\mu \mathrm{m}$ ) and relatively narrow particle size distributions (PSDs) were produced (Table S5). Such particles were expected to pack well into columns and to perform well as novel sorbents in SPE. 
Table S5. Particle sizes and particle size distributions of the polymer microspheres.

\begin{tabular}{cccc} 
& Particle size $^{\mathrm{a}}(\boldsymbol{\mu m})$ & $\mathbf{U}^{\mathrm{b}}$ & $\mathrm{CV}^{\mathrm{c}}(\%)$ \\
\hline MIP 1 & 2.8 & 1.203 & 28.2 \\
NIP 1 & 2.4 & 1.178 & 25.1 \\
MIP 2 & 2.8 & 1.090 & 19.4 \\
NIP 2 & 2.2 & 1.343 & 39.9 \\
\hline
\end{tabular}

${ }^{\mathrm{a}}$ Number-average particle size; ${ }^{\mathrm{b}}$ Polydispersity index; ${ }^{\mathrm{C}} \mathrm{Coefficient}$ of variation

\section{ANALYSIS OF POROUS MORPHOLOGY}

Specific surface areas, specific pore volumes, and mean pore sizes were assessed by using an ASAP 2000 BET Analyzer. For each analysis, around $250 \mathrm{mg}$ of polymer was dried overnight in a vacuum oven at $60{ }^{\circ} \mathrm{C}$, followed by a degassing step (pressure $\sim 3 \mathrm{mmHg}$ ) for $24 \mathrm{~h}$ at $100{ }^{\circ} \mathrm{C}$. The isotherms are presented in Fig. S4 and the computed data is presented in Table S6. 

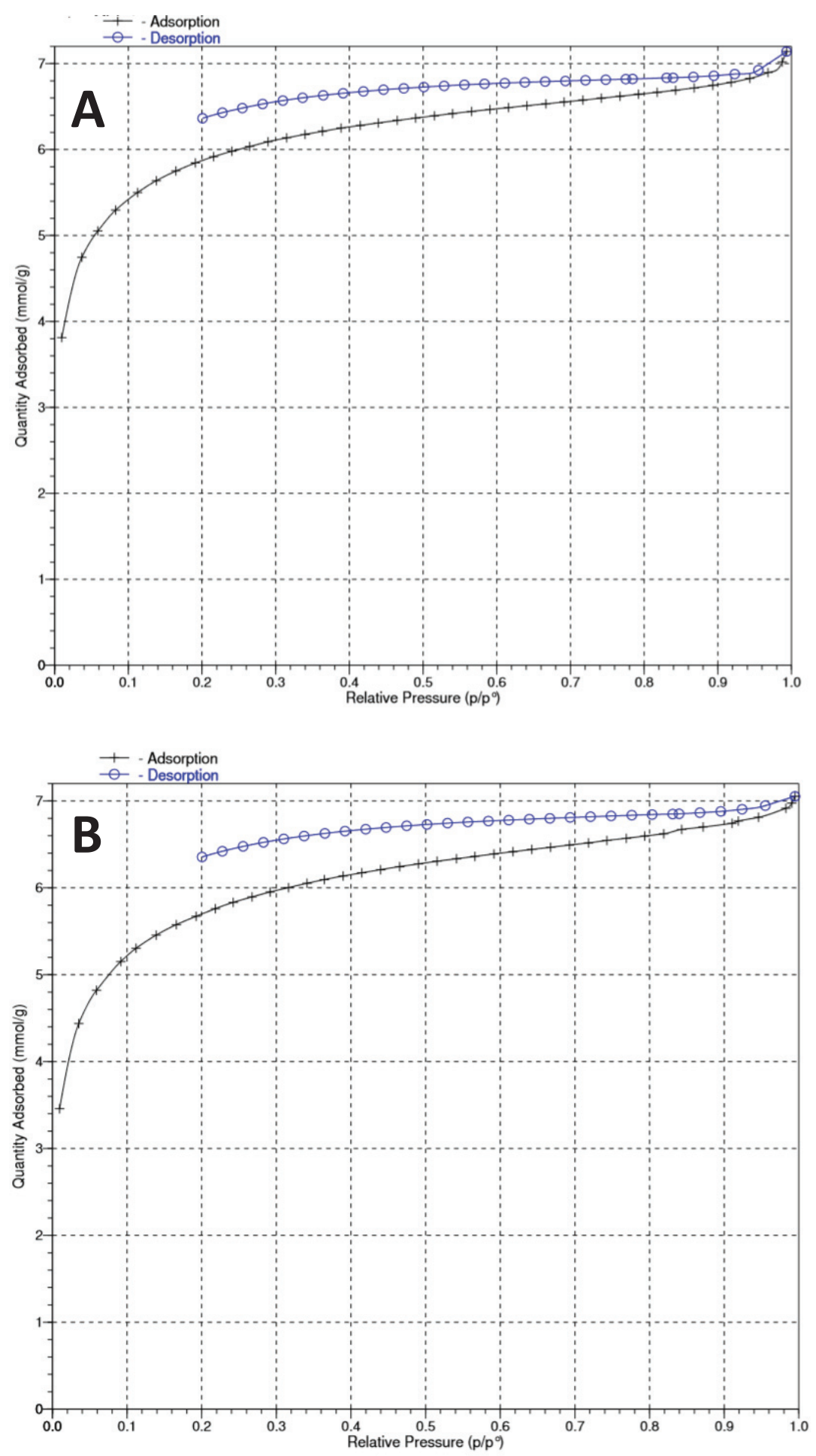

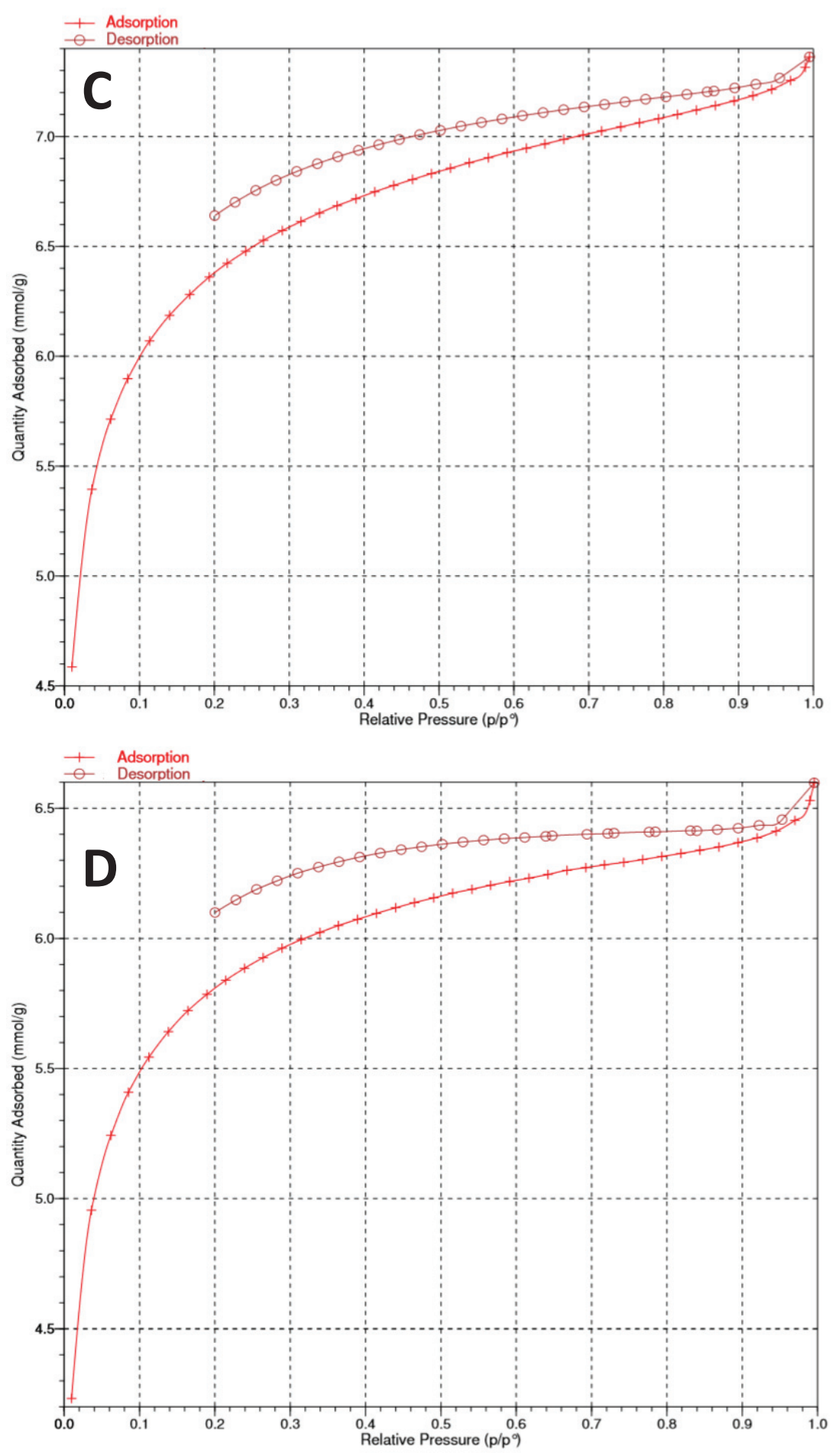

Figure S4. Nitrogen sorption analysis data for MIP 1 (A), NIP 1 (B), MIP 2 (C) and NIP 2 (D) 
Table S6. Specific surface areas, specific pore volumes and mean pore sizes of the MIPs and NIPs

\begin{tabular}{lccc}
\hline Polymer Code & $\begin{array}{c}\text { Langmuir specific } \\
\text { surface area }\left(\mathbf{m}^{2} / \mathbf{g}\right)\end{array}$ & $\begin{array}{c}\text { Specific pore volume } \\
\left(\mathbf{c m}^{3} / \mathbf{g}\right)\end{array}$ & Mean pore size $(\mathbf{n m})$ \\
\hline NIP 1 & 613 & 0.245 & 3.17 \\
MIP 1 & 623 & 0.248 & 2.99 \\
NIP 2 & 601 & 0.229 & 2.50 \\
MIP 2 & 664 & 0.255 & 2.50 \\
\hline
\end{tabular}

The use of THF gives rise to a well-developed pore structure in the polymer microspheres, as was reported in the literature. [3] As can be seen from the data in Table S5, all of the polymer networks are mesoporous (mean pore sizes are between 2-50 nm) and their pore characteristics are similar in terms of pore size, pore-volume and surface area. However, they are likely to contain a significant proportion of micropores too, which contributes to the relatively high values recorded for the specific surface areas. This is expected since the addition of THF as co-solvent changes the theta conditions and promotes porosity, in much the same way as toluene does for divinylbenzene copolymerisations. 


\section{REFERENCES}

[1] C. Rossetti, M.A. Świtnicka-Plak, T.G. Halvorsen, P.A.G. Cormack, B. Sellergren, L. Reubsaet, Automated Protein Biomarker Analysis: on-line extraction of clinical samples by Molecularly Imprinted Polymers, Sci. Rep. 7 (2017) 44298.

[2] J. Wang, P.A.G. Cormack, D.C. Sherrington, E. Khoshdel, Synthesis and characterization of micrometer-sized molecularly imprinted spherical polymer particulates prepared via precipitation polymerization, Pure Appl. Chem. 79(9) (2007) 1505-1519.

[3] F. Limé, K. Irgum, Preparation of divinylbenzene and divinylbenzene-co-glycidyl methacrylate particles by photoinitiated precipitation polymerization in different solvent mixtures,

Macromolecules 42(13) (2009) 4436-4442. 
